\title{
Combining information
}

Citation for published version (APA):

Cinar, O. (2021). Combining information: model selection in meta-analysis and methods for combining correlated $p$-values. [Doctoral Thesis, Maastricht University]. Ipskamp Printing BV. https://doi.org/10.26481/dis.20210519oc

Document status and date:

Published: 01/01/2021

DOI:

10.26481/dis.20210519oc

Document Version:

Publisher's PDF, also known as Version of record

\section{Please check the document version of this publication:}

- A submitted manuscript is the version of the article upon submission and before peer-review. There can be important differences between the submitted version and the official published version of record.

People interested in the research are advised to contact the author for the final version of the publication, or visit the DOI to the publisher's website.

- The final author version and the galley proof are versions of the publication after peer review.

- The final published version features the final layout of the paper including the volume, issue and page numbers.

Link to publication

\footnotetext{
General rights rights.

- You may freely distribute the URL identifying the publication in the public portal. please follow below link for the End User Agreement:

www.umlib.nl/taverne-license

Take down policy

If you believe that this document breaches copyright please contact us at:

repository@maastrichtuniversity.nl

providing details and we will investigate your claim.
}

Copyright and moral rights for the publications made accessible in the public portal are retained by the authors and/or other copyright owners and it is a condition of accessing publications that users recognise and abide by the legal requirements associated with these

- Users may download and print one copy of any publication from the public portal for the purpose of private study or research.

- You may not further distribute the material or use it for any profit-making activity or commercial gain

If the publication is distributed under the terms of Article $25 \mathrm{fa}$ of the Dutch Copyright Act, indicated by the "Taverne" license above, 
Doctoral thesis

\title{
COMBINING INFORMATION: MODEL SELECTION IN META-ANALYSIS AND METHODS FOR COMBINING CORRELATED P-VALUES
}

\author{
Ozan Çınar
}





\title{
COMBINING INFORMATION: MODEL SELECTION IN META-ANALYSIS AND METHODS FOR COMBINING CORRELATED P-VALUES
}

\author{
Dissertation \\ To obtain the degree of Doctor at Maastricht University, \\ on the authority of the Rector Magnificus, Prof. Dr. Rianne M. Letschert, \\ in accordance with the decision of the Board of Deans, \\ to be defended in public \\ on Wednesday 19th of May 2021, at 13.00 hours
}

by

Ozan Çınar 


\title{
Supervisor
}

Dr. W. Viechtbauer

\section{Co-supervisor}

Dr. I. S. Gülöksüz

\author{
Assessment Committee \\ Prof. dr. K.R.J. Schruers \\ Prof. dr. M.A.L.M. van Assen \\ Dr. M.J.J.M. Candel \\ Prof. dr. M. Dewey \\ Dr. Marjan Drukker
}

(C) Ozan Çınar, Maastricht 2021.

All rights reserved. No part of this publication may be reproduced, stored in a retrieval system or transmitted in any form or by any means, electronic, mechanical, photocopying, recording or otherwise, without prior written permission of the author.

Cover İpek Çınar and Alper Yıldırım, 2021

Production Ipskamp Printing || www.impskampprinting.nl ISBN 978-94-6423-216-5 


\section{Contents}

1 Introduction 3

1.1 Combining Information . . . . . . . . . . . . . 5

1.2 Heterogeneity and Model Selection in Meta-Regression . . . 6

1.3 Dependent and Multiple Effect Sizes . . . . . . . . . . . . 7

1.4 Combining Dependent $p$-Values . . . . . . . . . . . . . . 8

1.5 Aims and Outline of this Thesis . . . . . . . . . . . 9

References ...................... 11

2 Using information-theoretic approaches for model selection in meta$\begin{array}{ll}\text { analysis } & 17\end{array}$

2.1 Introduction . . . . . . . . . . . . . . . . . . 19

2.2 Meta-Analysis Models . . . . . . . . . . . . . . . 21

2.3 Model Selection . . . . . . . . . . . . . . . . . . . 24

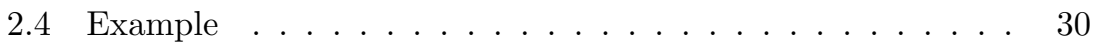

2.5 Simulation Study . . . . . . . . . . . . . . . . . . . . . . . . . . . . . . . . 34

2.6 Discussion ..................... . . 43

References ......................... 48

3 Phylogenetic multilevel meta-analysis: A simulation study on the importance of modeling the phylogeny $\quad 59$

3.1 Introduction . . . . . . . . . . . . . . . 61

3.2 Meta-Analytic Models . . . . . . . . . . . . . 63

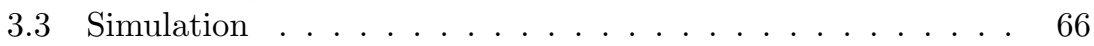

3.4 Discussion ......................... 73

References ....................... 78

4 A comparison of methods for gene-based testing that account for $\begin{array}{ll}\text { linkage disequilibrium } & 83\end{array}$

4.1 Introduction . . . . . . . . . . . . . . . . . . 85

4.2 Materials and Methods . . . . . . . . . . . . . . . 87

4.3 Results . . . . . . . . . . . . . . . . . . 99

4.4 Discussion ..................... . . . . 105

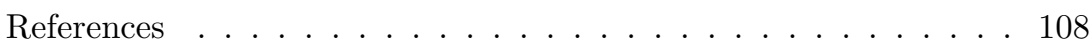


5 The poolr Package for Combining Independent and Dependent $p$ $\begin{array}{ll}\text { Values } & 123\end{array}$

5.1 Introduction . . . . . . . . . . . . . . . . . 125

5.2 Methods . . . . . . . . . . . . . . . . 127

5.3 Implementation . . . . . . . . . . . . . . . . . . . . . . . . 142

5.4 Example ...................... . . . 154

5.5 Comparison with other packages . . . . . . . . . 156

5.6 Conclusion . . . . . . . . . . . . . . . . 163

References ....................... 169

6 Discussion 175

6.1 Model Selection in Meta-Regression . . . . . . . . . . . . 176

6.2 Combining Information from Dependent Sources . . . . . . . 178

6.3 Tips for Analysts . . . . . . . . . . . . . . . . . . . . . 183

6.4 Limitations and possible future studies . . . . . . . . . . . . 184

References ..................... 187

7 General Summary 191

8 Impact on Society 195

$\begin{array}{ll}\text { Acknowledgments } & 197\end{array}$

$\begin{array}{ll}\text { About the author } & 201\end{array}$ 
List of abbreviations
AIC Akaike Information Criterion
BIC Bayesian Information Criterion
GWAS Genome-Wide Association Studies
IC Information Criterion
LD Linkage Disequilibrium
ML Maximum Likelihood
PCA Principal Component Analysis
REML Restricted Maximum Likelihood
RVI Relative Variance Importance
SNP Single-Nucleotide Polymorphism 



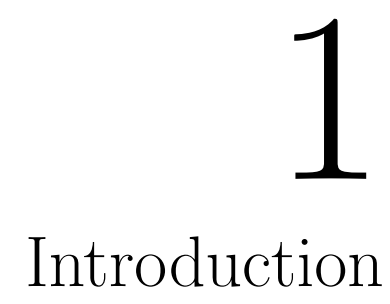


Knowledge is what we know

Also, what we know we do not know.

We discover what we do not know

Essentially by what we know.

Thus knowledge expands.

With more knowledge we come to know

More of what we do not know.

Thus knowledge expands endlessly.

These words ${ }^{1}$ by C. Radhakrishna Rao [1] gracefully illustrate the cumulative nature of science. Science, as a combination of various practices that include observation, testing, and questioning, is our essential tool to satisfy our curiosity about the nature around us and to unravel its secrets. Its cumulative nature reveals itself in the long run where we make discoveries wielding our prior knowledge. Through this continuous and cumulative process, we transcend our pool of knowledge which possibly is an infinite pool [2]. In a way, we climb the mountain of knowledge without knowing how far the crown of the mountain is in the clouds if there is a crown at all.

The cumulative nature of science has also influenced researchers to adopt a similar approach in the scientific literature. This was initiated by some researchers who pointed out the need to review the preceding scientific reports on a specific research question and synthesize them to derive a contemporary general answer [3]. For instance, Lord Rayleigh staunchly accentuated the need for such syntheses at the end of the 19th century [4]. He stated that science was at risk of being crushed under its weight due to the rapid growth of scientific reports and the lack of efforts to digest the preceding information. The efforts to address this issue then gave birth to several types of studies such as systematic reviews and meta-analysis, all of which have the goal of "combining information" [5].

\footnotetext{
${ }^{1}$ Although I am fully aware that this dissertation is not the place to discuss these words, I find them so impressive and meaningful, so the little scientist in me could not resist questioning them. If we define knowledge as the combination of what we know and we do not know, wouldn't we expand only the proportion of what we know in knowledge rather than expanding knowledge itself when we discover what we do not know by what we know? In my humble opinion, we do not expand the knowledge in the fifth line yet; however, it changes form. By increasing the proportion of what we know in knowledge, nevertheless, we do add more of what we do not know into knowledge; thus, knowledge expands continuously. Is it an endless expansion, this is another question in the part of what we do not know in our current knowledge.
} 


\subsection{Combining Information}

A research question is likely to be under investigation by multiple independent study groups, and one can easily encounter various reports on the same research question in the published literature whose conclusions most likely deviate qualitatively and quantitatively $[6,7]$. In this regard, combining information refers to an effort to review and summarize the results of the previous studies relevant to the research question. This effort can vary in practice from a basic narrative summary to the quantitative synthesis of the primary reports [8]. It can even appear in a form where experts gather and share their opinions to seek a consensus, a technique known as the Delphi method $[9,10]$.

Apart from reviewing and summarizing the literature, combining information can provide further benefits as the conclusions can be more precise and more generalizable than those of the primary studies, at least in theory $[5,11,12$, 13]. The development of methodology to reduce the imprecision in statistical analyses started in the early $20^{\text {th }}$ century in astronomy studies $[14,15]$ and was followed by further methods in medical studies [16]. Interestingly, current retrospective reviews also acknowledge that there was already a set of methodologies in physics and chemistry to improve the precision of the conclusions $[17,18,19,20]$.

The development of methodology to synthesize information quickened in the mid- $20^{t h}$ century. One of the major steps was taken in 1976 when Gene Glass formalized the notion of a "meta-analysis", a term that had been discussed among some researchers [21]. In his article, Glass proposed to classify scientific studies into primary and secondary analyses where the latter is a re-analysis of the former using other statistical techniques on the same data. Furthermore, he described a meta-analysis as an "analysis of analyses", a quantitative summary of the primary results derived by statistical methods. The introduction of this idea was followed by the influential works of others to diversify meta-analytic methods $[11,22,23]$.

As will be discussed later on, a traditional meta-analysis requires the quantitative results of the primary studies as input, usually in the form of "effect sizes" and their sampling variances. The sampling variances are required to weigh the effect sizes with respect to their precision measured by the sampling variances. However, the information to compute the effect sizes and the corresponding sampling variances may not always be available to the reviewer [24, 25]. In that case, one may have to resort to using methods for synthesizing information using more limited data, specifically the $p$-values that indicate 
the statistical significance of the results reported in the primary studies. This methodology is known as combining $p$-values and various methods have been described in the literature for this purpose $[26,27,28,29,30,31,32,33,34$, 35]. The common goal of these methods is to synthesize the $p$-values from multiple sources to test a global null hypothesis.

In essence, combining information, as a broad term including contexts like meta-analysis and combining $p$-values, relates the past knowledge to the future, longing to a more precise conclusion [5]. This goal may have a deeper meaning in the philosophy of science because such narrative or quantitative methods apply the cumulative nature of science to science itself. Maybe it is this meaning that led Gene Glass to conclude in his retrospective look on his personal history that meta-analysts do not publish studies; instead, they contribute to data archives [3].

Like other subjects, the process of combining information raises a vast amount of issues that need to be addressed. The main goal of this thesis is to study some of these issues in meta-analysis and methods for combining $p$-values.

\subsection{Heterogeneity and Model Selection in Meta-Regression}

Meta-analysis is a statistical study that employs quantitative methods to incorporate effect sizes from primary studies into an overall effect size and estimate its uncertainty [23]. This process requires the effect sizes reported by primary research that should have the same estimate type such as the standardized mean difference or odds ratio [36]. Even though the effect sizes are of the same type and answer the same specific research question, the true value that underlies the individual effect size estimates may deviate between studies, either due to randomness or differences in the study characteristics. This phenomenon is known as heterogeneity and needs to be carefully considered in a meta-analysis. For example, one can employ a random-effects model that assumes that the true values of the effect sizes follow a random distribution to account for the heterogeneity $[12,37]$. Furthermore, the heterogeneity and its significance can be assessed with statistics like $Q$ and $I^{2}[12,18,38,39,40]$.

Even if hypothesis tests suggest an insignificant amount of heterogeneity, it can still be argued that there is heterogeneity due to the clinical and methodological diversities among the primary studies [41]. This means that this heterogeneity can be incorporated and possibly explained by the characteristics 
of the primary studies by using regression-type techniques where different features of the primary studies are used as covariates, an analysis known as metaregression [41, 42, 43, 44, 45]. Meta-regression can address the systematic relationships between such study features and the effect sizes (hence, explain the heterogeneity) and result in more precise conclusions [41, 46].

An important issue in meta-regression is the sample size. It is defined by the number of primary studies which is usually much lower than the sample size in traditional regression analyses. Therefore, it is generally recommended to keep the number of covariates (known as "moderators" in the meta-regression context) low [41]. To do so, a meta-analyst can employ conventional model selection methods that are used in regression analyses. The most basic technique consists of testing each moderator individually to examine whether it has a significant association with the outcome of interest (possibly including the significant moderators in a final model). Another basic approach is to include all moderators together in a primary model, removing the insignificant moderators to derive some final model.

There are other principled model selection methods that are typically used in conventional regression analyses which can be applicable in meta-regression as well. For example, one can employ techniques based on information criteria such as the Akaike or Bayesian information criterion [47, 48, 49] to compare the likelihood of candidate models. One can also attempt to determine the "best" model by fitting all possible models and comparing them [48, 50], a process known as multimodel inference. However, these information-theoretic methods bring complexity into the analysis, and their performance relative to the aforementioned conventional techniques is unknown.

\subsection{Dependent and Multiple Effect Sizes}

An essential factor in meta-analysis is the independence assumption which implies that the effect sizes that are incorporated in the meta-analysis are assumed to be independent of each other. Although this assumption intuitively holds when the effect sizes are collected from independent primary studies, it can be easily violated in some specific contexts. For example, effect sizes in ecology studies are often derived from multiple species that share an evolutionary history known as a phylogeny [51, 52, 53]. Due to the phylogeny, the effect sizes synthesized in the meta-analysis cannot be considered to be independent of each other, and the independence assumption is violated. Phylogenies were first incorporated into meta-analyses in ecology with the methods 
introduced by Adams [54] and Lajeunesse [55] using fixed- and random-effects models, respectively. Soon thereafter, Chamberlain et al. [53] showed, based on a reanalysis of a series of published meta-analyses, that their conclusions can change when phylogenies are incorporated into the analyses.

Although a phylogeny can be appropriately incorporated into a meta-analysis with the aforementioned methods, another common issue in ecology studies is that the primary studies generally report multiple effect sizes. This introduces a within-study dependence and also violates the independence assumption. The models introduced by Adams and Lajeunesse are not appropriate when multiple effect sizes are reported. In such cases, a common practice is to summarize the effect sizes within primary studies into a single quantity and use these quantities in the meta-analysis. The downside of this practice, however, is a severe loss of information [36]. Hadfield and Nakagawa [56] proposed a multilevel meta-analytic model to address this issue which also incorporates the phylogeny in a similar manner proposed by Lynch [57], using two variance components for non-phylogenetic and phylogenetic relatedness among species. Although this model seems appropriate for incorporating both the multiple effect sizes and the phylogeny, it is a fairly complex model that may require large data sets to be used in practice [58].

\subsection{Combining Dependent $p$-Values}

Synthesizing information from multiple studies using meta-analytic techniques may require information not available to the reviewer (i.e., the effect size estimates and the corresponding sampling variances). In the absence of such data, conventional methods for synthesizing information may not be applicable. In such cases, one can employ methods for combining $p$-values that synthesize the individual $p$-values into a general value to test the global null hypothesis without the need of additional data. A vast range of methods for combining $p$-values have been described in the literature $[26,28,29,31,32,33,34,35$, 59].

Another common application of such methods arises when synthesizing information from multiple experimental units within a single study. One such use case arises in genome-wide association studies (GWAS). In GWAS, researchers collect data on genetic variants such as single-nucleotide polymorphisms (SNPs) on the genome [60]. Nowadays, GWAS often involve more than a million genetic variants to be tested simultaneously. Consequently, the Type I error rate can inflate very quickly due to such a large number of simultaneous 
tests. Although well-known multiple testing corrections such as the Bonferroni correction [61] can be applied to control the study-wise Type I error rate, with the extremely high number of simultaneous tests, multiple testing corrections may be too severe and will in turn increase the Type II error rate [62]. A promising approach to this problem is to shift the focus of the study to higher genetic levels like genes. Since gene regions cover SNPs on the genome, it is possible to test a gene that covers multiple SNPs as a global null hypothesis [60]. In other words, this approach combines the $p$-values of the SNPs that belong to a gene and tests the gene as a whole as a global null hypothesis.

Combining the SNP-level $p$-values to obtain a single $p$-value for a gene is known as gene-based testing [63]. With this approach, one can substantially decrease the number of multiple tests as the number of genes is much lower than the number of SNPs on the genome, which in turn may mend the aforementioned issues related to the Type I and II error rates [62, 64, 65, 66, 67]. Furthermore, a global test may be more appropriate theoretically to examine the genetic structures of complex diseases [67, 68].

While methods for combining $p$-values have been extensively studied, a common aspect of these methods is that they assume independence among the individual $p$-values. Similar to ecology studies, this assumption is violated in genetics as SNPs show nonrandom associations among each other, a phenomenon known as linkage disequilibrium [69]. Therefore, the SNP-level $p$ values are not independent of each other which violates the independence assumption. Ignoring the non-independence among the individual $p$-values may severely decrease the accuracy of the combined $p$-value [70]. Fortunately, it is possible to incorporate the dependence among the individual $p$-values into the process with various techniques, for example by estimating the effective number of tests [71, 72, 73, 74], using multivariate distributions [75, 76, 77], or permutation-based procedures [65, 67]. However, these methods introduce additional complexity into the analyses and require information about the nonindependence among the $p$-values, while their advantage is not entirely known. The statistical properties of these methods (in terms of their Type I error rate and power) have only been examined in parts.

\subsection{Aims and Outline of this Thesis}

As described above, there are several issues to be examined with respect to methods for meta-analysis and combining $p$-values. This thesis provides a review of these issues, suggests ways to address them, and evaluates various 
methods through simulation studies. Furthermore, examples from real data sets are used to illustrate the application of existing and novel methods.

Chapter 2 reviews methods for model selection in meta-regression and presents an example using real data to demonstrate the shortcomings of commonly used methods. Then, the results of a simulation study are described in which these methods were compared against more sophisticated ones based on information criteria.

Chapter 3 presents a simulation to examine the effects of incorporating dependence structures into meta-analytic methods. In particular, this study examines the performance of a multilevel meta-analytic model that addresses multiple effect sizes reported by primary studies and based on multiple species.

Chapter 4 reviews available methods for combining $p$-values along with adjustment techniques to incorporate the dependence among the $p$-values into the process. An example based on a primary study is presented to illustrate the consequences of incorporating the dependence structure into the analysis. Furthermore, a simulation study was conducted to evaluate the various methods.

Chapter 5 then presents a novel open-source software package that implements the methods described in Chapter 4. The theoretical background of the methods implemented is described, followed by a tutorial on how to use the methods implemented in the software.

Chapter 6 discusses the main findings of the work presented in this thesis. Furthermore, the impact of the results of the studies are presented and possible future directions are addressed.

Finally, Chapter 7 briefly summarizes the main findings of the studies included in this thesis.

With more knowledge we come to know

More of what we do not know.

Thus knowledge expands endlessly.

***

All knowledge is, in final analysis, history.

All sciences are, in the abstract, mathematics.

All judgements are, in their rationale, statistics. 


\section{References}

[1] C Radhakrishna Rao. Statistics and truth: putting chance to work. World Scientific, 1997.

[2] Richard P Feynman. "The Character of Physical Law. The 1964 Messenger Lectures". In: Cambridge MA (1965).

[3] Gene V Glass. "Meta-analysis at middle age: a personal history". In: Research Synthesis Methods 6.3 (2015), pp. 221-231.

[4] Clive E Adams, Stefanie Polzmacher, and Annabelle Wolff. "Systematic reviews: work that needs to be done and not to be done". In: Journal of Evidence-Based Medicine 6.4 (2013), pp. 232-235.

[5] Iain Chalmers, Larry V Hedges, and Harris Cooper. "A brief history of research synthesis". In: Evaluation $\&$ the health professions 25.1 (2002), pp. $12-37$.

[6] Phillipa J Easterbrook et al. "Publication bias in clinical research". In: The Lancet 337.8746 (1991), pp. 867-872.

[7] Hannah R Rothstein, Alexander J Sutton, and Michael Borenstein. "Publication bias in meta-analysis". In: Publication bias in meta-analysis: Prevention, assessment and adjustments (2005), pp. 1-7.

[8] Mark Petticrew. "Systematic reviews from astronomy to zoology: myths and misconceptions". In: Bmj 322.7278 (2001), pp. 98-101.

[9] Norman Crolee Dalkey. Studies in the Quality of Life; Delphi and Decision-Making. 1972.

[10] Juri Pill. "The Delphi method: substance, context, a critique and an annotated bibliography". In: Socio-economic planning sciences 5.1 (1971), pp. $57-71$.

[11] H Cooper and LV Hedges, eds. The handbook of research synthesis. Russell Sage Foundation, 1994.

[12] Larry Hedges and Ingram Olkin. Statistical models for meta-analysis. New York: Academic Press, 1985.

[13] Morton Hunt. How science takes stock: The story of meta-analysis. Russell Sage Foundation, 1997.

[14] George Biddell Airy. On the algebraical and numerical theory of errors of observations and the combination of observations. Macmillan\&Company, 1861. 
[15] Matthias Egger, George Davey Smith, and Keith O'Rourke. "Introduction: rationale, potentials, and promise of systematic reviews". In: Systematic reviews in health care: meta-analysis in context (2001), pp. 119.

[16] Karl Pearson. "Report on certain enteric fever inoculation statistics". In: British Medical Journal 3 (1904), pp. 1243-1246.

[17] Larry V Hedges. The early history of meta-analysis. 2015.

[18] Larry V Hedges. "The statistics of replication". In: Methodology (2019).

[19] Cho Yen Ho, Reginald W Powell, and Peter E Liley. "Thermal conductivity of the elements". In: Journal of Physical and Chemical Reference Data 1.2 (1972), pp. 279-421.

[20] Arthur H Rosenfeld. "The Particle Data Group: Growth and OperationsEighteen Years of Particle Physics". In: Annual Review of Nuclear Science 25.1 (1975), pp. 555-598.

[21] Gene V Glass. "Primary, secondary, and meta-analysis of research". In: Educational researcher 5.10 (1976), pp. 3-8.

[22] Thomas D Cook et al. Meta-analysis for explanation: A casebook. Russell Sage Foundation, 1992.

[23] H. Cooper, L. V. Hedges, and J. C. Valentine, eds. The handbook of research synthesis and meta-analysis. 2nd. New York: Russell Sage Foundation, 2009.

[24] Nicholas A Heard and Patrick Rubin-Delanchy. "Choosing between methods of combining-values". In: Biometrika 105.1 (2018), pp. 239-246.

[25] Walter W Piegorsch and A John Bailer. "Combining information". In: Wiley StatsRef: Statistics Reference Online (2014).

[26] William G Cochran. "Problems arising in the analysis of a series of similar experiments". In: Supplement to the Journal of the Royal Statistical Society 4.1 (1937), pp. 102-118.

[27] R. A. Fisher. Statistical Methods for Researchers (4th. ed.) Edinburgh, UK: Oliver and Boyd, 1932.

[28] H. O. Lancaster. "The Combination of Probabilities Arising from Data in Discrete Distributions". In: Biometrika 36.3/4 (1949), pp. 370-382. DOI: https://doi.org/10.1093/biomet/36.3-4.370.

[29] T Liptak. "On the combination of independent tests". In: Magyar Tud Akad Mat Kutato Int Kozl 3 (1958), pp. 171-197. 
[30] Karl Pearson. "On a method of determining whether a sample of size n supposed to have been drawn from a parent population having a known probability integral has probably been drawn at random". In: Biometrika (1933), pp. 379-410.

[31] E. S. Pearson. "The Probability Integral Transformation for Testing Goodness of Fit and Combining Independent Tests of Significance". In: Biometrika 30.1/2 (1938), pp. 134-148. DoI: https ://doi .org/10. $2307 / 2332229$.

[32] S. A. Stouffer et al. The American Soldier: Adjustment During Army Life (Studies in Social Psychology in World War II. Vol. 1. Princeton: Princeton University Press, 1949.

[33] L. H. C. Tippett. The Methods of Statistics. London: Williams \& Norgate, 1931 .

[34] B. Wilkinson. "A Statistical Consideration in Psychological Research". In: Psychological Bulletin 48.2 (1951), pp. 156-158. DOI: https://doi. org/10.1037/h0059111.

[35] F Yates and William G Cochran. "The analysis of groups of experiments". In: The Journal of Agricultural Science 28.4 (1938), pp. 556-580.

[36] Shinichi Nakagawa and Eduardo SA Santos. "Methodological issues and advances in biological meta-analysis". In: Evolutionary Ecology 26.5 (2012), pp. 1253-1274.

[37] Larry V Hedges and Jack L Vevea. "Fixed-and random-effects models in meta-analysis." In: Psychological methods 3.4 (1998), p. 486.

[38] Julian PT Higgins and Simon G Thompson. "Quantifying heterogeneity in a meta-analysis". In: Statistics in medicine 21.11 (2002), pp. 15391558 .

[39] Julian PT Higgins et al. "Measuring inconsistency in meta-analyses". In: Bmj 327.7414 (2003), pp. 557-560.

[40] Larry V Hedges and Therese D Pigott. "The power of statistical tests for moderators in meta-analysis". In: Psychological methods 9.4 (2004), p. 426 .

[41] S. G. Thompson and J. P. T. Higgins. "How should meta-regression analyses be undertaken and interpreted?" In: Statistics in Medicine 21.11 (2002), pp. 1559-1573.

[42] C. S. Berkey et al. "A random-effects regression model for meta-analysis". In: Statistics in Medicine 14.4 (1995), pp. 395-411. 
[43] H. C. van Houwelingen, L. R. Arends, and T. Stijnen. "Advanced methods in meta-analysis: Multivariate approach and meta-regression". In: Statistics in Medicine 21.4 (2002), pp. 589-624.

[44] S. W. Raudenbush. "Analyzing effect sizes: Random-effects models". In: The handbook of research synthesis and meta-analysis. Ed. by H. Cooper, L. V. Hedges, and J. C. Valentine. 2nd. New York: Russell Sage Foundation, 2009, pp. 295-315.

[45] S. G. Thompson and S. J. Sharp. "Explaining heterogeneity in metaanalysis: A comparison of methods". In: Statistics in Medicine 18.20 (1999), pp. 2693-2708.

[46] Julia Koricheva and Jessica Gurevitch. "Uses and misuses of meta-analysis in plant ecology". In: Journal of Ecology 102.4 (2014), pp. 828-844.

[47] D. R. Anderson. Model based inference in the life sciences: A primer on evidence. 2nd. New York: Springer, 2007.

[48] K. P. Burnham and D. R. Anderson. Model selection and multimodel inference: A practical information-theoretic approach. 2nd. New York: Springer, 2002.

[49] C. Chatfield. "Model uncertainty, data mining and statistical inference". In: Journal of the Royal Statistical Society, Series A 158.3 (1995), pp. 419-466.

[50] P. M. Lukacs, K. P. Burnham, and D. R. Anderson. "Model selection bias and Freedman's paradox". In: Annals of the Institute of Statistical Mathematics 62.1 (2010), pp. 117-125.

[51] Göran Arnqvist and David Wooster. "Meta-analysis: synthesizing research findings in ecology and evolution". In: Trends in Ecology 83 Evolution 10.6 (1995), pp. 236-240.

[52] Jessica Gurevitch and Larry V Hedges. "Statistical issues in ecological meta-analyses". In: Ecology 80.4 (1999), pp. 1142-1149.

[53] S. A. Chamberlain et al. "Does phylogeny matter? Assessing the impact of phylogenetic information in ecological meta-analysis". In: Ecology Letters 15.6 (2012), pp. 627-36.

[54] Dean C. Adams. "Phylogenetic meta-analysis". In: Evolution 62.3 (Mar. 2008), pp. 567-572. DOI: $10.1111 / \mathrm{j} .1558-5646.2007 .00314 . x$.

[55] Marc J Lajeunesse. "Meta-analysis and the comparative phylogenetic method". In: The American Naturalist 174.3 (2009), pp. 369-381. 
[56] Jarrod D Hadfield and Shinichi Nakagawa. "General quantitative genetic methods for comparative biology: phylogenies, taxonomies and multitrait models for continuous and categorical characters". In: Journal of evolutionary biology 23.3 (2010), pp. 494-508.

[57] Michael Lynch. "Methods for the analysis of comparative data in evolutionary biology". In: Evolution 45.5 (1991), pp. 1065-1080.

[58] Douglas Bates et al. "Parsimonious mixed models". In: arXiv preprint arXiv:1506.04967 (2015).

[59] Ronald Aylmer Fisher et al. "Statistical methods for research workers." In: Statistical methods for research workers. 5th Ed (1934).

[60] B. Lehne, C. M. Lewis, and T. Schlitt. "From SNPs to Genes: disease Association at the Gene Level". In: PLoS One 6.6 (2011), e20133. DOI: https://doi.org/10.1371/journal.pone.0020133.

[61] J. M. Bland and D. G. Altman. "Multiple Significance Tests: The Bonferroni Method". In: British Medical Journal 310.6973 (1995), p. 170. DOI: https://doi.org/10.1136/bmj .310.6973.170.

[62] R. C. Johnson et al. "Accounting for Multiple Comparisons in a GenomeWide Association Study (GWAS)". In: BMC Genomics 11.1 (2010), p. 724. DOI: https://doi.org/10.1186/1471-2164-11-724.

[63] H. Huang et al. "Gene-Based Tests of Association". In: (2011). DoI: 10. 1371/journal10.1371/journal.pgen.1002177.t001.

[64] S. E. Baranzini et al. "Pathway and network-based analysis of genomewide association studies in multiple sclerosis". In: Hum Mol Genet 18.11 (2009), pp. 2078-90. DOI: 10.1093/hmg/ddp120.

[65] K. N. Conneely and M. Boehnke. "So many correlated tests, so little time! Rapid adjustment of P values for multiple correlated tests". In: Am J Hum Genet 81.6 (2007), pp. 1158-68. DOI: 10.1086/522036.

[66] X. Hu et al. "Group-combined P-values with applications to genetic association studies". In: Bioinformatics 32.18 (2016), pp. 2737-43. DOI: 10.1093/bioinformatics/btw314.

[67] J. Z. Liu et al. "A Versatile Gene-Based Test for Genome-Wide Association Studies". In: The American Journal of Human Genetics 87.1 (2010), pp. 139-145. DOI: https://doi.org/10.1016/j.ajhg.2010.06.009.

[68] Jaeyoon Chung et al. "Comparison of methods for multivariate genebased association tests for complex diseases using common variants". In: European Journal of Human Genetics (2019), p. 1. DOI: https://doi. org/10.1038/s41431-018-0327-8. 
[69] M. Slatkin. "Linkage Disequilibrium-Understanding the Evolutionary Past and Mapping the Medical Future". In: Nature Reviews Genetics 9.6 (2008), pp. 477-485. DOI: https://doi.org/10.1038/nrg2361.

[70] V. Moskvina et al. "Evaluation of an Approximation Method for Assessment of Overall Significance of Multiple Dependent Tests in a Genomewide Association Study". In: Genetic Epidemiology 35.8 (2011), pp. 861-866. DOI: https://doi.org/10.1002/gepi. 20636.

[71] D. R. Nyholt. "A simple correction for multiple testing for single-nucleotide polymorphisms in linkage disequilibrium with each other". In: Am J Hum Genet 74.4 (2004), pp. 765-9. DOI: 10.1086/383251.

[72] J. Li and L. Ji. "Adjusting multiple testing in multilocus analyses using the eigenvalues of a correlation matrix". In: Heredity (Edinb) 95.3 (2005), pp. 221-7. DOI: $10.1038 / \mathrm{sj}$.hdy .6800717.

[73] X. Gao, J. Starmer, and E. R. Martin. "A multiple testing correction method for genetic association studies using correlated single nucleotide polymorphisms". In: Genet Epidemiol 32.4 (2008), pp. 361-9. DOI: 10. 1002/gepi.20310.

[74] N. W. Galwey. "A new measure of the effective number of tests, a practical tool for comparing families of non-independent significance tests". In: Genet Epidemiol 33.7 (2009), pp. 559-68. DOI: 10.1002/gepi. 20408.

[75] M. J. Strube. "Combining and Comparing Significance Levels from Nonindependent Hypothesis Tests". In: Psychological Bulletin 97.2 (1985), pp. 334-341. DOI: https://doi.org/10.1037/0033-2909.97.2.334.

[76] M. B. Brown. "400: A Method for Combining Non-Independent, OneSided Tests of Significance". In: Biometrics (1975), pp. 987-992. DOI: https://doi.org/10.2307/2529826.

[77] James J Yang et al. "An efficient genome-wide association test for multivariate phenotypes based on the Fisher combination function". In: $B M C$ bioinformatics 17.1 (2016), p. 19. 


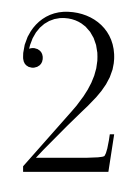

\section{Using information-theoretic approaches for model selection in meta-analysis}

Ozan Cinar et al. "Using information-theoretic approaches for model selection in meta-analysis". In: ((Submitted to) Research Synthesis Methods). 


\section{Abstract}

Meta-regression examines the association between the effect size estimates and the characteristics of the studies included in a meta-analysis using regressiontype methods. By searching for those characteristics (i.e., moderators) that are related to the effect sizes, we seek to identify a model that represents the best approximation to the underlying data generating mechanism. Model selection via testing, either through a series of univariate models or a model including all moderators, is the most commonly used approach for this purpose. Here, we describe alternative model selection methods based on information criteria, multimodel inference, and relative variable importance. We demonstrate their application using an illustrative example, and present results from a simulation study to compare the performance of the various model selection methods for identifying the true model across a wide variety of conditions. Whether information-theoretic approaches can also be used not only in combination with maximum likelihood (ML) but also restricted maximum likelihood (REML) estimation was also examined. The results indicate that the conventional methods for model selection may be outperformed by information-theoretic approaches. The latter are more often among the set of best methods across all of the conditions simulated and can have higher probabilities for identifying the true model under particular scenarios. Moreover, their performance based on REML estimation was either very similar to that from ML estimation or at times even better depending on how exactly the REML likelihood was computed. These results suggest that alternative model selection methods should be more widely applied in meta-regression. 


\subsection{Introduction}

Empirical research often involves taking measurements to assess the magnitude of a treatment effect, the size of a group difference, or the direction and strength of the relationship between two variables (i.e., an 'effect size'). When multiple estimates of a phenomenon of interest are available from a collection of studies, meta-analytic methods can be used to synthesize the estimates into an overall value that typically will be more precise than the individual estimates [e.g., 1]. In addition, since the aggregated value is usually a reflection of estimates obtained under varying conditions and circumstances, it carries with it a sense of greater generalizability.

At the same time, one can also examine whether the effect sizes are systematically related to the conditions and circumstances under which they were obtained. Such moderator analyses are commonly conducted by means of an approach called meta-regression [e.g., 2, 3, 4, 5, 6]. Here, one first codes the various (study-level) characteristics of interest into a set of predictor variables or 'moderators'. Next, regression-type models are used to examine the relationship between moderators and the estimates.

In practice, meta-analytic datasets tend to be highly multi-factorial in nature, containing a large number of study characteristics that are potentially relevant and plausible predictors of the observed effects [6]. Moreover, such analyses are inherently observational in nature because the values of the explanatory variables have not been independently/systematically manipulated [6]. As a result, some combinations of study characteristics are likely to be much more common than other combinations, typically leading to correlation and incomplete orthogonality among the explanatory variables.

Nevertheless, it is still common practice to examine one moderator variable at a time by means of a series of univariate or 'single-factor' meta-regression models [7]. However, unless steps are taken to control the family-wise Type I error rate (which is also not common practice in this context), doing so leads to a high chance of false positive findings. Moreover, due to non-orthogonality, moderator variables found to be relevant predictors are likely to account for shared variability in the effect sizes. Fitting meta-regression models containing multiple predictors of interest may circumvent the latter issue, but it is unclear how moderators should be selected for inclusion in such multi-factor models. Model selection based on stepwise procedures and drawing (dichotomous) conclusions about the relevance of moderators based on null hypothesis testing and p-values has repeatedly been argued to be of limited value [e.g., 
8, 9]. Also, parameter estimates and the statistical significance of particular moderators may vary substantially among models, depending on which other moderators are included.

Although typically not framed in this manner, fitting meta-regression models is in essence a form of model selection. Accordingly, one could also consider to make use of methods for model selection and multimodel inference based on information-theoretic approaches [e.g., 8, 10, 11]. However, it is far from straightforward to apply information criteria in model selection in this context. To start, it is not clear which estimation procedure to use for model fitting (e.g., ML, REML), nor which information criterion to choose (e.g., AIC, BIC, $\mathrm{AICc})$. On the one hand, it has been argued that information criteria under REML (restricted maximum likelihood) estimation should not be used to compare models differing in their fixed effects [e.g., 12, 13] because the contrasts used to derive the restricted likelihood (which is then used in the calculation of the information criteria) depend on the model matrix of the fixed effects. However, simulation results from Gurka [14] suggest that this problem may not be substantial for some types of models and datasets. Moreover, we might prefer to use REML over ML (maximum likelihood) estimation, since the latter may produce biased parameter estimates (especially for variance components in the model), which in turn affect the calculation of the ICs [15]. We are aware of two simulation studies exploring these issues [14, 16], but results from those studies are inconclusive and only narrowly related to the models and data structures used in meta-analyses.

The purpose of this paper is therefore to examine methods for model selection when faced with multi-factorial data in the meta-analytic context. We are specifically interested in how well methods based on information criteria and multimodel inference compare against simpler methods such as univariate testing of individual moderator variables or fitting a single model including all potential moderator variables of interest. To examine these issues, we conducted a simulation study comparing the various approaches using data reflective of a wide variety of circumstances one may encounter in practice.

The outline of the paper is as follows. In section 2.2 , we describe the metaanalytic random- and mixed-effects meta-regression models, ML and REML estimation thereof, and standard methods for testing coefficients in the context of these models. In section 2.3, model selection via testing, information criteria, multimodel inference, and relative variable importance are described. In section 2.4, we then provide an example illustrating the various approaches based on a dataset containing the results from studies examining the effects 
of inoculation with root-symbiotic mycorrhizal fungi on plant biomass. The methods and results for the simulation study comparing the various approaches are described in section 2.5. We then conclude the paper with a discussion of the findings in section 2.6, where we address some additional issues and concerns.

\subsection{Meta-Analysis Models}

We assume that a collection of $k$ independent studies has been selected for inclusion in a meta-analysis and that each study provides a single effect size estimate or observed outcome. For example, for a set of studies examining the effectiveness of a particular experimental treatment, the outcome may be the (standardized) mean difference or log response ratio [17]. When examining the relationship between two variables, the outcome may be the raw or Fisher's r-to-z transformed correlation coefficient [17]. In the health/medical sciences, dependent variables are often measured dichotomously, leading to (log-transformed) odds/risk ratios and risk differences as effect size measures of choice [18].

Regardless of the outcome measure used, let $y_{i}$ denote the observed value in the $i$ th study and $\theta_{i}$ the corresponding true parameter. For brevity, we will refer to $y_{i}$ as the effect size estimate and $\theta_{i}$ as the true effect size. We assume that

$$
y_{i}=\theta_{i}+\epsilon_{i},
$$

where $\epsilon_{i} \sim N\left(0, v_{i}\right)$ denotes the sampling error and $v_{i}$ the sampling variance of the $i$ th estimate. For all of the effect size measures commonly used in metaanalysis (and all of the measures noted above), we can derive an estimate of $v_{i}$ for each study $[17,18]$. The sampling variances are typically treated as known constants in the analyses (even though technically some uncertainty is still attached to them, especially in small samples).

\subsubsection{Random- and Mixed-Effects Models}

According to the random-effects model [e.g., 4], the true effect sizes are heterogeneous and are given by

$$
\theta_{i}=\mu+u_{i},
$$

where $u_{i} \sim N\left(0, \tau^{2}\right)$. Therefore, $\tau^{2}$ denotes the amount of heterogeneity in the true effects and $E\left[\theta_{i}\right]=\mu$ the average true effect. A special case of the 
random-effects model arises when $\tau^{2}=0$, in which case the true effects are homogeneous.

Heterogeneity in the true effects is often not purely random, as assumed by the random-effects model, but a result of systematic differences between the studies (e.g., in terms of how an experimental treatment was implemented). Assume that information about $p$ potential moderator variables has been extracted from the studies along with the effect size estimates. We can then set up a mixed-effects meta-regression model of the form

$$
\theta_{i}=\beta_{0}+\beta_{1} x_{i 1}+\ldots+\beta_{p} x_{i p}+u_{i}
$$

where $x_{i j}$ denotes the observed value of the $j$ th moderator variable in the $i$ the study, $\beta_{j}$ denotes how $E\left[\theta_{i}\right]$ changes for a one-unit increase in $x_{i j}$, and $u_{i} \sim N\left(0, \tau^{2}\right)$ as before, but $\tau^{2}$ now denotes residual heterogeneity, that is, variability in the true effects not accounted for by the moderators included in the model [e.g., 2, 3, 4, 5, 6].

The meta-regression model above can accommodate (a mixture of) quantitative and qualitative moderator variables (the latter through appropriate dummy coding of the various levels of the factor) and $x_{i j}$ may also reflect an interaction term between two or more moderator variables or polynomial/spline functions of individual moderators (to model the non-linear influence of a quantitative moderator variable on the effect sizes). However, we will only consider main effects throughout this paper, as models involving higher-order terms are not frequently used in practice.

\subsubsection{Model Fitting and Inference}

Let $\boldsymbol{X}$ denote the $(k \times(p+1))$ model matrix containing the values of the $p$ moderator variables with a vector of ones in the first column, corresponding to the model intercept. In fact, the random-effects model is just a special case of the mixed-effects model, where $\boldsymbol{X}$ simply consists of a column of ones. Next, let $\boldsymbol{y}$ denote the $(k \times 1)$ vector with the observed effect size estimates and $\boldsymbol{V}$ a $(k \times k)$ diagonal matrix with the sampling variances (i.e., the $v_{i}$ values) along the diagonal. The random/mixed-effects model can then be written as

$$
\boldsymbol{y} \sim N(\boldsymbol{X} \boldsymbol{\beta}, \boldsymbol{M})
$$


where $\boldsymbol{M}=\boldsymbol{V}+\tau^{2} \boldsymbol{I}$ and $\boldsymbol{I}$ denotes a $(k \times k)$ identity matrix. Letting $\boldsymbol{W}=$ $\boldsymbol{M}^{-1}$, the $\log$ likelihood function is therefore given by

$$
l l_{M L}\left(\boldsymbol{\beta}, \tau^{2}\right)=-\frac{k}{2} \ln (2 \pi)-\frac{1}{2} \ln |\boldsymbol{M}|-\frac{1}{2}(\boldsymbol{y}-\boldsymbol{X} \boldsymbol{\beta})^{\prime} \boldsymbol{W}(\boldsymbol{y}-\boldsymbol{X} \boldsymbol{\beta}),
$$

which depends on $\boldsymbol{\beta}$ and $\tau^{2}$. For a given value of $\tau^{2}$, the maximum likelihood estimate of $\boldsymbol{\beta}$ is given by

$$
\boldsymbol{b}=\left(\boldsymbol{X}^{\prime} \boldsymbol{W} \boldsymbol{X}\right)^{-1} \boldsymbol{X}^{\prime} \boldsymbol{W} \boldsymbol{y} .
$$

Hence, finding the maximum likelihood estimates of $\boldsymbol{\beta}$ and $\tau^{2}$ is considerably simplified by maximizing the profiled log likelihood

$$
l l_{M L}\left(\tau^{2}\right)=-\frac{k}{2} \ln (2 \pi)-\frac{1}{2} \ln |\boldsymbol{M}|-\frac{1}{2}(\boldsymbol{y}-\boldsymbol{X} \boldsymbol{b})^{\prime} \boldsymbol{W}(\boldsymbol{y}-\boldsymbol{X} \boldsymbol{b})
$$

over $\tau^{2}$ and then obtaining the maximum likelihood estimates of the elements in $\boldsymbol{\beta}$ with (2.6).

Maximum likelihood estimates of $\tau^{2}$ are known to be negatively biased in small samples (i.e., when $k$ is small) [19]. On the other hand, restricted maximum likelihood (REML) estimation yields (approximately) unbiased estimates and is therefore to be preferred when unbiasedness is deemed important. The restricted log likelihood function is given by

$$
\begin{aligned}
l l_{R E M L}\left(\tau^{2}\right) & =-\frac{k-p-1}{2} \ln (2 \pi)+\frac{1}{2} \ln \left|\boldsymbol{X}^{\prime} \boldsymbol{X}\right| \\
& -\frac{1}{2} \ln |\boldsymbol{M}|-\frac{1}{2} \ln \left|\boldsymbol{X}^{\prime} \boldsymbol{W} \boldsymbol{X}\right|-\frac{1}{2} \boldsymbol{y}^{\prime} \boldsymbol{P} \boldsymbol{y}
\end{aligned}
$$

where

$$
\boldsymbol{P}=\boldsymbol{W}-\boldsymbol{W} \boldsymbol{X}\left(\boldsymbol{X}^{\prime} \boldsymbol{W} \boldsymbol{X}\right)^{-1} \boldsymbol{X}^{\prime} \boldsymbol{W} .
$$

Since the REML likelihood only depends on $\tau^{2}$, maximization of $l l_{R E M L}\left(\tau^{2}\right)$ is again a one-dimensional optimization problem. Once the REML estimate of $\tau^{2}$ has been obtained, we can again estimate the elements in $\boldsymbol{\beta}$ with (2.6).

Maximization of (2.7) and (2.8) can be easily accomplished either by an exhaustive search or by means of an optimization algorithm, such as gradient ascent or some Newton-type algorithm [20]. Below, we will denote the maximized values of (2.7) and (2.8) as $l l_{M L}$ and $l l_{R E M L}$, respectively, and the estimate of $\tau^{2}$ that is obtained with either method by $\hat{\tau}^{2}$. 
Once $\tau^{2}$ has been estimated with either ML or REML estimation, the estimated model coefficients (i.e., $b_{0}, b_{1}, \ldots, b_{p}$ ) are then given by $(2.6)$, with $\hat{\tau}^{2}$ substituted for $\tau^{2}$ in $\boldsymbol{M}$ and hence $\boldsymbol{W}$. The variance-covariance matrix of the model coefficients can then be estimated with

$$
\operatorname{Var}[\boldsymbol{b}]=\left(\boldsymbol{X}^{\prime} \boldsymbol{W} \boldsymbol{X}\right)^{-1}
$$

The diagonal elements of $\operatorname{Var}[\boldsymbol{b}]$ contain the estimated sampling variances of the model coefficients (i.e., $\operatorname{Var}\left[b_{0}\right], \operatorname{Var}\left[b_{1}\right], \ldots, \operatorname{Var}\left[b_{p}\right]$ ). Taking the square-root thereof provides the estimated standard errors (i.e., $\left.\mathrm{SE}\left[b_{0}\right], \mathrm{SE}\left[b_{1}\right], \ldots, \mathrm{SE}\left[b_{p}\right]\right)$. A Wald-type test of an individual moderator variable [e.g., 4] can then be conducted by comparing

$$
z_{j}=\frac{b_{j}}{\mathrm{SE}\left[b_{j}\right]}
$$

against the critical bounds of a standard normal distribution (e.g., \pm 1.96 for $\alpha=.05$, two-sided). Values of $z_{j}$ equal to or larger than the critical values lead to the rejection of $H_{0}: \beta_{j}=0$. Analogously, approximate $95 \%$ confidence intervals for the coefficients can be constructed with

$$
b_{j} \pm 1.96 \mathrm{SE}\left[b_{j}\right]
$$

Some alternative methods for making inferences about the coefficients in metaregression models have been developed [for a summary and comparison, see e.g., 21], but we will not consider these methods further here.

\subsection{Model Selection}

In practice, one often faces the problem that a large number of moderator variables have been measured, but it is unclear which of these moderators are actually related to the effect sizes. The problem of model selection in metaregression can therefore be stated as follows. Let $p$ denote the total number of moderators of interest and that could be included in the model. Now suppose $m(0 \leq m \leq p)$ of these moderators actually exert an influence on the effect sizes, while the remaining $p-m$ moderators do not. Let $T \subseteq\{1, \ldots, p\}$ denote the set with the $m$ indices of the true moderators. Then the true model is given by

$$
y_{i}=\beta_{0}+\sum_{j \in T} \beta_{j} x_{i j}+u_{i}+\epsilon_{i}
$$


while the full model, containing 'true' and 'false' moderators, is given by

$$
y_{i}=\beta_{0}+\sum_{j \in T} \beta_{j} x_{i j}+\sum_{j \notin T} \beta_{j} x_{i j}+u_{i}+\epsilon_{i},
$$

where, in truth, $\beta_{j}=0$ for $j \notin T$. The goal is then to identify model (2.13) as the true model via some kind of model selection method.

We should clarify that model (2.13) is assumed to be the true model only in the sense that it reflects the best approximation to a much more complicated reality given the available data. The actual data generating mechanism is going to include moderators that have not actually been measured and/or coded and may not even be a linear model of the type described above. In addition, the moderators that have been coded are often just proxies or surrogates for the actual variables involved in the data generating mechanism. Therefore, whenever we refer to the true model and the true/false moderators, these terms should always be thought of in quotes.

\subsubsection{Model Selection Methods}

We will consider four general approaches for identifying the set of true moderators and hence model (2.13) as the true model. The first approach includes simple methods that are commonly used in practice, namely univariate testing of the $p$ moderator variables or testing of the $p$ moderators in the context of the full model. The second approach examines the complete set of $2^{p}$ models that can be fitted given the $p$ moderator variables and then uses various information criteria for model selection $[8,10]$. Next, we consider methods based on model averaging and multimodel inference over the set of $2^{p}$ models to identify the true moderators $[8,10]$. Finally, based on the information criteria, one can compute a 'relative variable importance' for each of the moderators of interest, which can then be used for model selection. The various methods are described in more detail below.

\subsubsection{Selection via Testing}

In practice, relatively simple methods are commonly used for model selection in the meta-regression context. We will consider two such approaches based on Wald-type tests of the model coefficients. In the first approach, one simply fits $p$ univariate meta-regression models (i.e., including one moderator variable at a time) and obtains the corresponding Wald-type tests of the coefficients. 
Based on the significance of these $p$ tests, moderators are classified as either true or false. In the second approach, the full model including all $p$ moderators is fitted. Again, Wald-type tests (now in the context of the full model) are used to categorize the moderator variables as being related to the effect sizes or not. With either testing approach, we can consider the true model as identified if we reject $H_{0}: \beta_{j}=0$ for all $j \in T$ and fail to reject $H_{0}: \beta_{j}=0$ for all $j \notin T$. We do not consider the possibility of applying corrections for multiple testing (e.g., the Bonferroni correction), as this is not common practice in the meta-analytic context [7].

\subsubsection{Selection via Information Criteria}

Based on the $p$ moderator variables, a total of $R=2^{p}$ models can be fitted to the given data. While the true model should, on average, provide the best fit, we cannot use the log likelihoods directly for model selection, as the likelihood always increases as more moderators are added to the model. On the other hand, information criteria, which penalize the maximized likelihoods for model complexity, can be used for this purpose. The 'best fitting' model (in the sense of making a trade-off between model fit and model complexity) is the one that minimizes a particular information criterion. From an information-theoretic perspective, model selection based on information criteria can also be described as a way for minimizing the loss of information (in a Kullback-Leibler sense) when approximating full reality by a fitted model $[8,10]$.

The most commonly known criterion for this purpose is the Akaike Information Criterion [22], which is given by

$$
A I C=-2 l l+2(s+2),
$$

where $l l$ is either $l l_{M L}$ or $l l_{R E M L}$, depending on the estimation method used, and $s(0 \leq s \leq p)$ denotes the number of moderators included in the model (hence, the model contains $s+2$ parameters, counting the model intercept and $\left.\tau^{2}\right)$.

Another commonly used criterion is the Bayesian Information Criterion [23], which is given by

$$
B I C=-2 l l+(s+2) \ln \left(k^{*}\right),
$$

where $k^{*}=k$ for ML estimation and $k^{*}=k-s-1$ for REML estimation. When $k^{*} \geq 8$, the BIC penalizes the model fit more heavily than the AIC and therefore should tend to select models with fewer fixed effects. 
Finally, we will consider a third criterion, a finite sample size (second-order bias) corrected version of the AIC [24, 25], given by

$$
A I C c=-2 l l+2(s+2)\left(\frac{k^{*}}{k^{*}-(s+2)-1}\right),
$$

where $k^{*}=\max (k, s+4)$ for ML estimation and $k^{*}=\max (k-s-1, s+$ 4) for REML estimation (these definitions of $k^{*}$ ensure that the additional multiplicative factor in the $A I C c$ is always $\geq 1)$. As $k /(s+2)$ increases (i.e., the ratio of sample size to the number of parameters), $A I C c$ converges to the $A I C$ from above. However, in situations where $k$ is small relative to the number of parameters, $A I C c$ will again tend to favor models with fewer fixed effects when compared to the $A I C$.

Regardless of the criterion, we then simply consider the true model as identified if it corresponds to the best model, that is, the model with the smallest value for the chosen information criterion. However, several issues are of note here when using REML estimation for model selection. First, the restricted log likelihood function (2.8) is obtained by taking linear combinations (contrasts) of the elements in $\boldsymbol{y}$, such that the transformed data are free of the fixed effects in $\boldsymbol{\beta}$ [e.g., 26, 27]. Since the appropriate transformation depends on $\boldsymbol{X}$, models with different fixed effects will require different transformations, leading to restricted likelihoods that are technically not directly comparable. Consequently, likelihood ratio tests based on (2.8) are not appropriate for comparing models with different fixed effects [e.g., 12, 13]. However, some recent simulations [14] suggest that model selection based on information criteria computed with (2.8) may still be a valid strategy.

Second, while the restricted log likelihood function technically only contains one unknown parameter (i.e., $\tau^{2}$ ), we do count the regression coefficients for the moderators as additional parameters when computing the information criteria under REML estimation, as otherwise we would always select the full model as the optimal one.

Finally, note that the second term (i.e., $\frac{1}{2} \ln \left|\boldsymbol{X}^{\prime} \boldsymbol{X}\right|$ ) in the restricted log likelihood function (2.8) does not depend on $\tau^{2}$ (or $\boldsymbol{\beta}$ ) and hence is irrelevant for maximizing the restricted log likelihood. Consequently, this term is often omitted by software when computing and reporting $l l_{R E M L}$. However, when using REML estimation for model comparisons involving different sets of moderators, the relevance of including this term in the computation of $l l_{R E M L}$ is unclear [14]. Therefore, we define the maximized log likelihood with and with- 

meta-analysis

out the second term in (2.8) as $l l_{R E M L f}$ and $l l_{R E M L r}$, respectively, and can compute the information criteria with respect to both values.

\subsubsection{Selection via Multimodel Inference}

Approaches that simply test coefficients (either univariately or in the context of the full model) do not take model uncertainty into consideration. As an alternative, we can base our inferences on all available models, using model averaging to obtain parameter estimates (and corresponding standard errors) that properly reflect this uncertainty. This multimodel inference approach works as follows.

First, based on a particular information criterion (e.g., AIC based on ML estimation), we estimate the probability that each of the $R=2^{p}$ models in the candidate set is the best model (in a Kullback-Leibler sense) with

$$
w_{r}=\frac{\exp \left(-\frac{1}{2} \Delta_{r}\right)}{\sum_{r=1}^{R} \exp \left(-\frac{1}{2} \Delta_{r}\right)},
$$

where $\Delta_{r}=I C_{r}-I C_{\min }, I C_{r}$ denotes the information criterion for the $r$ th model, and $I C_{\min }$ the value of the information criterion for the model with the smallest value (hence $\Delta_{r}=0$ for the model selected as the best model based on the chosen information criterion). These model probabilities are also commonly referred to as Akaike weights and, given particular priors, reflect posterior model probabilities in a Bayesian framework $[8,10]$.

Next, model averaged parameter estimates for each coefficient are obtained with

$$
\bar{b}_{j}=\sum_{r=1}^{R} w_{r} b_{r j},
$$

where $b_{r j}$ denotes the estimated value of $\beta_{j}$ in the $r$ th model. Therefore, the estimated model coefficient for the $j$ th moderator variable is then based on the entire collection of $R$ models, with weights assigned in accordance to the estimated probabilities that each model is in fact the best model.

When computing $\bar{b}_{j}$, a decision needs to be made how to handle models that actually do not contain the $j$ th moderator as one of the predictor variables (when considering all $2^{p}$ models, only half of the models will actually contain the $j$ th moderator). Two strategies are commonly used to handle this $[8,28]$ : When computing $\bar{b}_{j}$, we can only consider the subset of models that actually 
estimated $\beta_{j}$ (doing so requires renormalizing the model weights, so that they sum to 1 for a given subset) or alternatively we can set the coefficient equal to zero for models that do not actually estimate $\beta_{j}$. The latter approach can be motivated on two grounds. First, the omission of a particular predictor from a model is in essence equivalent to assigning a value of zero to the corresponding coefficient. Second, setting the coefficient to zero in models that omit the $j$ th moderator variable results in shrinkage of the model averaged parameter estimate that may counteract model selection bias [10]. We therefore only consider the second approach, but return to this issue in the discussion.

An estimate of the variance of the model averaged parameter estimate accounting for both sampling error and model uncertainty can then be obtained with

$$
\operatorname{Var}\left[\bar{b}_{j}\right]=\sum_{r=1}^{R} w_{r}\left(\operatorname{Var}\left[b_{r j}\right]+\left(b_{r j}-\bar{b}_{j}\right)^{2}\right),
$$

where $\operatorname{Var}\left[b_{r j}\right]$ denotes the estimated sampling variance of the $j$ th model coefficient from the $r$ th model [10]. When the $r$ th model does not actually contain the $j$ th model coefficient, then $\operatorname{Var}\left[b_{r j}\right]=0$ (and $b_{r j}=0$ ). The square-root of (2.20) provides an estimate of the standard error of the model averaged parameter estimate (i.e., $\left.\mathrm{SE}\left[\bar{b}_{j}\right]\right)$.

Finally, we can draw inferences about the relevance of each model coefficient, using (2.19) and (2.20) to construct the test statistic

$$
\bar{z}_{j}=\frac{\bar{b}_{j}}{\mathrm{SE}\left[\bar{b}_{j}\right]},
$$

which we compare against the critical bounds of a standard normal distribution (e.g., \pm 1.96 for $\alpha=.05$, two-sided). Values of $\bar{z}_{j}$ equal to or larger than the critical values are again taken as evidence that the $j$ th moderator variable is related to the effect sizes and is therefore classified as a true moderator.

\subsubsection{Selection via Relative Variable Importance}

Instead of testing, we can also use the Akaike weight of each model to calculate the relative importance of each moderator, that is, the relative variable importance (RVI) across the candidate set of models, which for a particular moderator is the sum of the Akaike weights of all models in which that moderator occurs. Formally, letting $I_{r j}=1$ if the $j$ th moderator is included in the 
$r$ th model and 0 otherwise,

$$
R V I_{j}=\sum_{r=1}^{R} w_{r} I_{r j}
$$

is the sum of the Akaike weights for the $j$ th moderator for all models in which the moderator appears. In practice, a sufficiently high RVI (often 0.5 or 0.8 ) is taken as evidence that a particular moderator is valuable for inference, although we are not aware of strong theoretical support for using any particular RVI criterion. Here, we explore both 0.5 and 0.8 as criteria, in each case taking an RVI greater than or equal to those values as evidence that a moderator is related to the effect sizes. As in testing, if $R V I_{j} \geq 0.5$ (or 0.8 ) for all $j \in T$ and $R V I_{j}<0.5$ (or 0.8 ) for all $j \notin T$, we consider the true model as identified.

\subsection{Example}

As an illustration, we applied the various approaches described above to a dataset of 80 effect size estimates obtained from studies examining the influence on plant biomass of inoculation with mycorrhizal fungi, which are soilinhabiting root symbionts of plants that have the potential to improve plant growth. The dataset and $\mathrm{R}$ code to reproduce the following analyses are available at the Open Science Framework (https://osf.io/3d8u5/). Note that the dataset is used for illustration purposes only and is actually part of a much larger database [29], including over 4000 estimates. Here, we focus on a subset of the data containing only estimates for corn, i.e., the plant Zea mays, inoculated with one of two different genera of arbuscular mycorrhizal (AM) fungi, either Funneliformis or Rhizophagus. The subset was selected to avoid some of the additional complexities in the actual data structure (e.g., many plant species and fungi, phylogenetic relatedness, nesting and non-independence due to multiple estimates obtained from the same study and/or a shared control condition). The outcome measure used for the meta-analysis is the log response ratio [30], with positive values reflecting an increase in mean biomass in plants receiving the mycorrhizal inoculation compared to non-inoculated plants.

A random-effects model fitted to the data using REML estimation yields an aggregated effect size estimate of $\hat{\mu}=0.59$ (with $95 \%$ CI: 0.37 to 0.80 ). After exponentiation, we therefore estimate that mycorrhizal inoculation increases plant biomass on average by a factor of 1.80 (i.e., $80 \%$ ) (95\% CI: 1.45 to 2.23 ). 
However, there appears to be considerable heterogeneity in the effect sizes, with $\tau^{2}$ being estimated at 0.523 in this model.

A large number of variables may systematically influence plant response to inoculation, but we focus here on 4 potential moderators. Besides the mycorrhizal fungus (FUN) used ( $k$ for Funneliformis: $29 ; k$ for Rhizophagus: 51 ), the dataset includes information about whether phosphorus fertilizer was added (FP; $k$ for no: $54 ; k$ for yes: 26 ), whether nitrogen fertilizer was added (FN; $k$ for no: $22 ; k$ for yes: 58 ), and whether or not the background soil was sterilized prior to mycorrhizal inoculation (STER; $k$ for no: $16 ; k$ for yes: 64 ). Due to the observational nature of these data, the four factors were only partially crossed, with some combinations being much more prevalent than others (and some not represented at all). Computing correlations (phi coefficients) after dummy-coding each factor yielded values in the range of -.14 to .53 . See Table 2.1 for the full correlation matrix.

Table 1: Correlation matrix (phi coefficients) of the four dichotomous moderator variables.

\begin{tabular}{ccccc}
\hline & FUN & FP & FN & STER \\
FUN & 1 & -0.14 & 0.53 & 0.27 \\
FP & -0.14 & 1 & 0.31 & 0.01 \\
FN & 0.53 & 0.31 & 1 & 0.53 \\
STER & 0.27 & 0.01 & 0.53 & 1 \\
\hline
\end{tabular}

Table 2.2 shows the values of the $A I C c$ and model probabilities (i.e., $w_{r}$ ) for the $2^{4}=16$ possible models (considering only main effects and no interactions) when using REML estimation for the model fitting and computing the maximized restricted log-likelihood with $l_{R E M L r}$. The model with the lowest $A I C c$ includes moderators $\mathrm{FN}$ and $\mathrm{FP}$, with an associated model probability of 0.47 . Therefore, based on model selection via information criteria, we would consider moderators $\mathrm{FN}$ and FP as relevant.

Table 2.3 shows the results when testing each moderator in a series of univariate models, when testing each moderator in the context of the full model (i.e., with all four predictors included simultaneously), and when testing each moderator using multimodel inference using model averaged parameter estimates and corresponding standard errors along with their relative variance importance values based on all 16 models. From Table 2.2, we can see how the coefficient and standard error of the FP moderator is obtained for each of these approaches (see model 8 for the univariate model approach, model 6 
Chapter 2. Using information-theoretic approaches for model selection in meta-analysis

Table 2: Value of the AICc (based on $l l_{R E M L r}$ ) for the 16 models fitted to the data examining the influence of mycorrhizal inoculation on plant biomass

\begin{tabular}{crcccc}
\hline Model & Moderator $(\mathrm{s})$ & $A I C c$ & $w_{r}$ & $b_{\mathrm{FP}}$ & $\mathrm{SE}\left[b_{\mathrm{FP}}\right]$ \\
\hline 1 & $\mathrm{FP}+\mathrm{FN}$ & 203.210 & 0.47 & -1.00 & 0.207 \\
2 & $\mathrm{FP}+\mathrm{FN}+\mathrm{STER}$ & 205.161 & 0.18 & -0.97 & 0.209 \\
3 & $\mathrm{FUN}+\mathrm{FP}+\mathrm{FN}$ & 205.507 & 0.15 & -0.92 & 0.224 \\
4 & $\mathrm{FUN}+\mathrm{FP}+\mathrm{STER}$ & 207.540 & 0.05 & -0.71 & 0.206 \\
5 & $\mathrm{FUN}+\mathrm{FP}$ & 207.566 & 0.05 & -0.66 & 0.208 \\
6 & $\mathrm{FUN}+\mathrm{FP}+\mathrm{FN}+\mathrm{STER}$ & 207.611 & 0.05 & $-0.90^{(a)}$ & 0.226 \\
7 & $\mathrm{FP}+\mathrm{STER}$ & 208.534 & 0.03 & -0.75 & 0.209 \\
8 & $\mathrm{FP}$ & 212.524 & 0.00 & $-0.71^{(b)}$ & 0.220 \\
9 & $\mathrm{FUN}$ & 213.047 & 0.00 & 0.00 & 0.000 \\
10 & $\mathrm{FUN}+\mathrm{STER}$ & 214.157 & 0.00 & 0.00 & 0.000 \\
11 & $\mathrm{FUN}+\mathrm{FN}$ & 215.739 & 0.00 & 0.00 & 0.000 \\
12 & $\mathrm{STER}$ & 216.281 & 0.00 & 0.00 & 0.000 \\
13 & $\mathrm{FUN}+\mathrm{FN}+\mathrm{STER}$ & 216.765 & 0.00 & 0.00 & 0.000 \\
14 & $\mathrm{FN}$ & 217.803 & 0.00 & 0.00 & 0.000 \\
15 & $\mathrm{FN}+\mathrm{STER}$ & 218.329 & 0.00 & 0.00 & 0.000 \\
16 & - & 218.485 & 0.00 & 0.00 & 0.000 \\
\hline Avg & & & & $-0.93^{(c)}$ & 0.248 \\
\hline
\end{tabular}

(a) estimated model coefficient for moderator FP from the model including all moderators simultaneously; $(b)$ estimated coefficient for moderator FP from the univariate model; $(c)$ model-averaged parameter estimate (and corresponding standard error) for moderator FP.

when testing moderators in the context of the full model, and the value in the last row for the multimodel inference approach). Univariate testing leads to the conclusion that all four moderators are related to the effect sizes. When testing the moderators in the context of the full model and when using the multimodel inference approach, we come to a very different conclusion, only finding support for the relevance of FP as a potential moderator.

Clearly, there is substantial support for the importance of FP, with the predictor appearing in the top 8 models as ranked by the $A I C c$ (the model probabilities for these 8 models add up to 0.99 , which is therefore also the relative variable importance for this moderator). Similarly, all testing methods identify this variable as a relevant predictor. Support for the relevance of the other moderators is weaker, although FN is part of the top three models as ranked by 


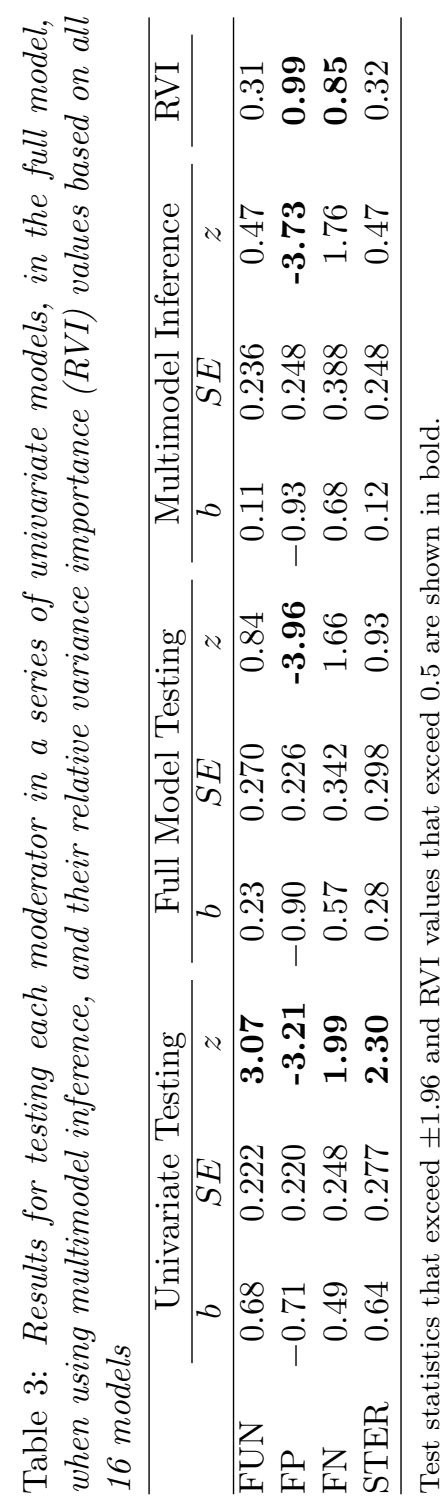


the $A I C c$, with a relative variable importance of 0.85 . Also, univariate testing (just barely) finds this moderator to be significant. The remaining moderators, FUN and STER, are not found to be significant by full model testing or multimodel inference. Furthermore, their relative variance importance are 0.31 and 0.32 , respectively, both of which suggest that these moderators are unrelated to the effect sizes.

The example illustrates that conclusions regarding the relevance of particular moderators can depend on the method used for model selection. To determine whether a particular approach is preferable (in terms of being more likely to select the true model), we conducted a simulation study.

\subsection{Simulation Study}

Given the choice among the different approaches (and the resulting potential for conflicting conclusions), we conducted a Monte Carlo simulation study to compare the accuracy of the various methods for identifying the true model under ML and REML estimation. We also examined the relevance of using $l l_{R E M L f}$ versus $l l_{R E M L r}$ for computing the restricted log likelihood when using the various information criteria for model selection. In the present section, we describe the methods used for the simulation study and the results obtained.

\subsubsection{Methods}

Assume that each study included in the meta-analysis compared two experimental groups with respect to a quantitative dependent variable. We will use the mean difference as the effect size measure for the meta-analysis, which is given by $y_{i}=\bar{x}_{i 1}-\bar{x}_{i 2}$, where $\bar{x}_{i 1}$ and $\bar{x}_{i 2}$ denote the observed means of the dependent variable in the first (e.g., treated) and second (e.g., non-treated) group. Then $y_{i} \sim N\left(\theta_{i}, \sigma_{i}^{2}\left(1 / n_{i 1}+1 / n_{i 2}\right)\right)$, where $\theta_{i}=\mu_{i 1}-\mu_{i 2}$ is the true effect in the $i$ th study, $\mu_{i 1}$ and $\mu_{i 2}$ denote the true means of the two experimental groups, $n_{i 1}$ and $n_{i 2}$ the group sizes, and $\sigma_{i}^{2}$ the true variance of the dependent variable in an individual study. Without loss of generality, we set $\mu_{i 2}=0, \sigma_{i}^{2}=1$, and assume $n_{i 1}=n_{i 2} \equiv n_{i}$.

In each iteration of the simulation, we first simulated the sample sizes of the individual studies by drawing $k$ values from $\chi_{d f=4}^{2}$, a chi-square distribution with 4 degrees of freedom, and then letting $n_{i}=\bar{n}\left(\left(\chi_{d f=4}^{2}+6\right) / 20\right)$ (rounded to the nearest integer), so that $\bar{n}$ denotes the average (total) sample size of each 
study included in the meta-analysis. The sample size distributions generated in this manner have a minimum of $\bar{n}(3 / 10)$, a mean of $\bar{n} / 2$, and are right-skewed as typically encountered in practice.

Next, we simulated the values of the moderator variables by drawing $k$ sets of $p$ values from a multivariate standard normal distribution, where all $p$ variables (i.e., the true and false moderators) were correlated with each other with correlation equal to $\rho$. When $\rho=0$, the simulated moderator variables were uncorrelated, while values of $\rho \neq 0$ correspond to the more commonly encountered situation where moderator variables are correlated with each other.

Finally, given the sample sizes and the moderator values, we then simulated the $k$ observed effect sizes from $y_{i} \sim N\left(\sum_{j=1}^{m} \beta_{j} x_{i j}, \tau^{2}+2 / n_{i}\right)$ for a given value of $\tau^{2}$, so that moderators $j=\{1, \ldots, m\}$ are the true and $j=\{m+1, \ldots, p\}$ are the false moderators. To keep the number of conditions manageable, we set $\beta_{j}=\beta$ for $j=1$ to $m$. Therefore, each of the $m$ true moderators exerted the same amount of influence on the effect sizes. On the other hand, the remaining $p-m$ false moderators did not influence the effect sizes.

Within each iteration, we therefore obtained $k$ values of $y_{i}$, the corresponding sampling variances $v_{i}=2 / n_{i}$, and the values of the $p$ moderator variables corresponding to each of the $k$ studies. The various model selection approaches described earlier were then used in an attempt to identify the true model. Therefore, we fitted each of the $2^{p}$ possible models to the data using ML and REML estimation and recorded the corresponding AIC, BIC, and AICc values. For REML estimation, both $l l_{R E M L f}$ and $l l_{R E M L r}$ were used for computing the restricted log likelihood and the resulting information criteria. We then recorded whether the true model was correctly selected according to the minimum AIC, BIC, and AICc criteria.

For the testing approaches, we recorded whether the set of significant and nonsignificant moderators corresponded to the set of true and false moderators in the true model, respectively. If all of the true moderators and none of the false moderators were significant, then this was considered a correct identification of the true model. The same approach was used when using the RVI of the variables as the selection method (using either 0.5 or 0.8 as the cutoff for considering a variable as important).

The following factors were examined in the simulation study: $k(20,30,40$, $60,80), \bar{n}(15,30,60,120), \rho(0,0.3,0.6), \beta(0,0.1,0.2,0.3,0.4), \tau^{2}(0$, $\left.0.1^{2}, 0.2^{2}, 0.3^{2}, 0.4^{2}\right), p(4,6,8,10)$ and $m$ (either $m$ was set equal to $p / 2$ 
or held constant at $m=2$ ). All factors were fully crossed, so that a total of $5 \times 4 \times 3 \times 5 \times 5 \times 4 \times 2=12,000$ conditions were examined (note that when $\beta=0$, then the true model is actually the 'empty model' that does not include any moderators, regardless of the value of $m$ ). For each condition, we simulated 1000 meta-analyses and then estimated the probability of identifying the true model by computing the proportion of iterations in which the true model was correctly identified by each of the methods described above.

For the design of the simulation study above, it can be shown that the total amount of heterogeneity in the true effects is equal to $\operatorname{Var}\left[\theta_{i}\right]=m(1+(m-$ 1) $\rho) \beta^{2}+\tau^{2}$. Furthermore, note that, on average, $n_{i}=\bar{n} / 2$, so that, on average, $\bar{v}=4 / \bar{n}$. Based on these values, we can compute $I^{2}=\operatorname{Var}\left[\theta_{i}\right] /\left(\operatorname{Var}\left[\theta_{i}\right]+\bar{v}\right)$, that is, how much of the total amount of variability in the effect size estimates can, on average, be attributed to heterogeneity in the true effects. Therefore, when $\beta=0$, the values of $I^{2}$ corresponding to the $\bar{n}$ and $\tau^{2}$ values chosen for the simulation study range from 0 to .38 (with a median of .13) for $\bar{n}=15$ and from 0 to .83 (with a median of .55) for $\bar{n}=120$. When $\beta>0$, the resulting $I^{2}$ values increase in accordance with $\beta, m, \rho$, and $\tau^{2}$. Values of $I^{2}$ around $.25, .50$, and .75 are typically considered to reflect low, moderate, and high amounts of heterogeneity [31], but when multiple strong moderators are exerting an influence on the effect sizes, values above .90 are not uncommon. Therefore, the values of the factors above were chosen in accordance with these considerations.

Furthermore, the simulation design implies that the amount of heterogeneity that can be accounted for by any individual true moderator is equal to $R^{2}=\beta^{2} /\left(m(1+(m-1) \rho) \beta^{2}+\tau^{2}\right)$. Therefore, as $m, \rho$, and $\tau^{2}$ increase, the explanatory power of each individual moderator decreases. However, the total amount of heterogeneity that can be accounted for by all true moderators increases with $m$ and $\rho$ and is simply equal to 1 when $\tau^{2}=0$.

The simulation study was programmed in $\mathrm{R}$ [32]. Due to the large number of conditions and the highly intensive nature of the computations (especially when fitting all $2^{p}$ models), the simulation study was programmed to make use of multicore processing using parallelization. The simulation was run on a cluster computer using 126 cores simultaneously for the computations. Even making use of such computing power, completion time for the entire set of conditions was a bit over 5 days (roughly 16,000 core hours in total). 


\subsubsection{Results}

Given the large number of conditions and selection methods examined in the simulation, summarizing the findings is challenging. To obtain a broad overview, we start by presenting kernel density plots of the probabilities of identifying the true model across all conditions for each of the methods in Figure 2.1 (40 different methods in total). The two lines in each plot distinguish the 2,400 conditions where the true model was the empty model (grey lines) from the 9,600 conditions where there was a non-zero association between the moderators and the effect sizes (black lines). The gray and black triangles in the plots correspond to the respective median probabilities. To make comparisons between these two classes of conditions and across methods easier, the densities were rescaled to have a maximum of 1 .

Selection via univariate or full model testing showed adequate performance when the true model was the empty model (no plots are shown for $l l_{R E M L r}$ because these methods are not affected by the way the REML likelihood is computed). In many such conditions, these methods correctly identified the empty model in at least $50 \%$ of the cases and typically even with higher probabilities (the median probabilities were close to $75 \%$ ). On the other hand, when there was an association between the moderators and the effect sizes, univariate testing had very low chances of identifying the true model in most conditions. Full model testing showed more promising performance. Here, the density was bimodal and appears to be composed of two 'sub-densities', the first peaking just above $0 \%$, which corresponds to conditions where identification of the true model is particularly difficult (i.e., various combinations of low $k$, low $\bar{n}$, high $\rho$, low $\beta$, high $\tau^{2}$, and high $p$ ). The other part of the density peaks around $80 \%$, but is quite left-skewed.

Several general conclusion can be reached about the methods based on information criteria. First, their performance was quite similar when computed based on $l l_{M L}$ or $l l_{R E M L f}$. Second, using the BIC was better at correctly identifying the empty model compared to the AIC, with the AICc typically falling in-between these two criteria at least in terms of the median performance. Moreover, all multimodel inference methods and all methods based on $l l_{R E M L r}$ performed exceptionally well in identifying the true model when none of the moderators were related to the effect sizes. Third, when the true model was not the empty model, performance of the various methods varied quite a bit, although we also typically see the bimodal shape in the densities as described above. In fact, for some methods, the bimodality was even more 

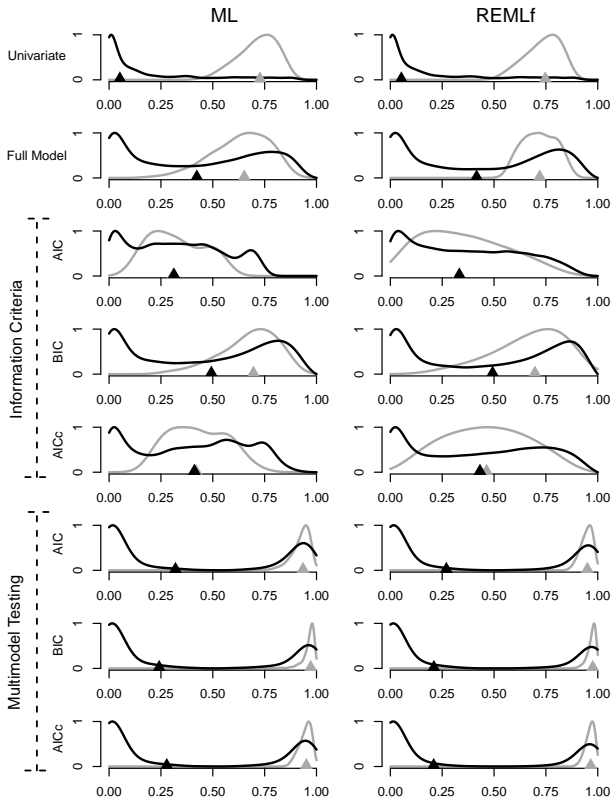

REMLr
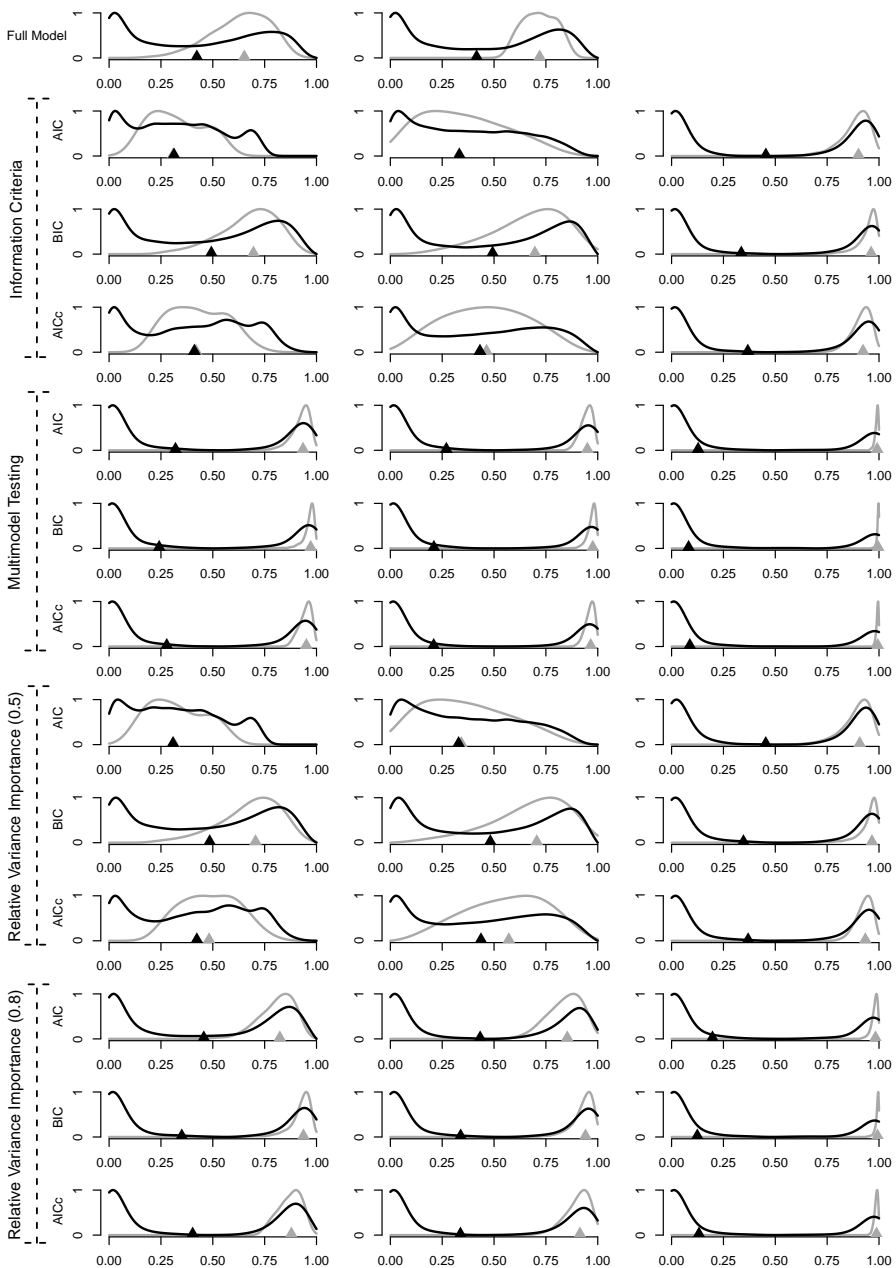

Figure 2.1: Kernel density plots of the probabilities of identifying the true model across all 12,000 conditions for each of the methods. Grey lines represent conditions where the true model was the empty model (i.e., $\beta=0$ ), whereas black lines represent conditions where there was a non-zero association between the moderators and the effect sizes (i.e., $\beta>0$ ). The triangles ( $\boldsymbol{\Delta}$ and $\boldsymbol{\Delta}$ ) indicate the corresponding median probabilities. 
pronounced. In other words, depending on the condition, there was then either a very low or a very high probability of correctly identifying the set of moderators that were truly related to the effect sizes.

To more directly compare the model selection methods against each other, we determined the method that obtained the highest probability of identifying the true model for each condition. However, a particular method may turn out to be the best for a particular condition simply due to simulation error. Moreover, the best method may outperform other methods just by a small margin, which could be considered practically irrelevant. Therefore, for each condition, we determined the set of methods that were no more than 5 percentage points worse than the best method (e.g., if the best method had a .38 probability of identifying the true model in a particular condition, then all methods that had at least a .33 probability were among the set of best methods for this condition). ${ }^{1}$ We then computed the proportion of conditions in which each method was among the best method (again separately for conditions where $\beta=0$ and where $\beta>0$ ). The results are shown in Figure 2.2.

When the true model was the empty model, univariate and full model testing were never among the best methods in any of the conditions. On the other hand, some of the information criteria methods were quite often among the best methods, especially when computed based on the $l l_{R E M L r}$ function. In that case, using multimodel inference and the RVI with a cutoff value of 0.8 was always or almost always among the best performing methods for identifying the empty model, regardless of whether the AIC, BIC, or AICc was used. On the other hand, when selecting based on the minimum of the information criteria or an RVI with a cutoff value of 0.5 , we again see that the BIC performed best, followed by the AICc and the AIC.

When the true model was not the empty model, univariate and full model testing were among the best methods for up to $20 \%$ of the conditions. However, the information-theoretic approaches always outperformed selection via univariate or full model testing, falling among the best methods for $26 \%$ to up to $49 \%$ of the conditions. While the specific information criterion and likelihood function played a relatively minor role in these results, selection via minimum information criteria or an RVI with a cutoff value of 0.5 tended to be among the best methods more often than selection via multimodel inference or when using an RVI with a cutoff value of 0.8 .

\footnotetext{
${ }^{1}$ The .05 margin is admittedly arbitrary, but slight variations in the margin did not materially affect the conclusions.
} 


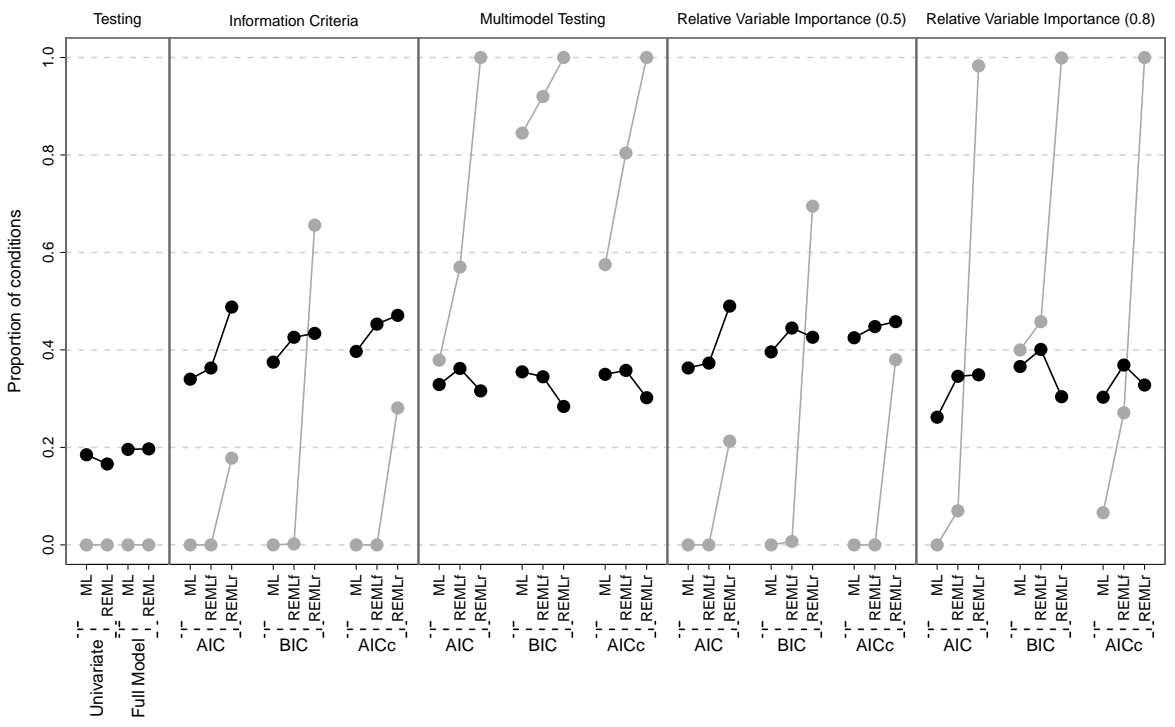

Figure 2.2: Proportion of conditions where each method was among the best methods (i.e., no worse than 5 percentage points than the best method). Grey lines represent conditions where the true model was the empty model (i.e., $\beta=0$ ), whereas black lines represent conditions where there was a non-zero association between the moderators and the effect sizes (i.e., $\beta>0$ ).

To determine the factors that are most relevant for accounting for the differences between the various selection methods, we structured the results from the simulation study as a dataset with 12,000 (for conditions) $\times 40$ (for methods $)=480,000$ rows and then conducted a two-way analysis of variance (ANOVA) with the (arcsine square-root transformed) probabilities of identifying the true model as the outcome variable (this data set is also available at OSF, https://osf.io/3d8u5/). The selection method, all design factors of the simulation, and their interactions with the selection method were included as predictors in the model. Table 2.4 presents the $\eta^{2}$ values of the main effects (along the diagonal) and the two-way interactions (the off-diagonal elements). The most influential factor was the size of the coefficient of the 
Table 4: $\eta^{2}$ values derived from the two-way ANOVA predicting the probabilities of selecting the true model based on the model selection method, all design factors, and their two-way interactions. The diagonal and off-diagonal values display the $\eta^{2}$ values of the main effects and two-way interactions, respectively.

\begin{tabular}{c|cccccccccc} 
Method & 0.27 & & & & & & & \\
$k$ & 0.10 & 0.47 & & & & & & \\
$\bar{n}$ & 0.07 & 0.01 & 0.42 & & & & & \\
$\rho$ & 0.05 & 0.00 & 0.00 & 0.18 & & & & \\
$\beta$ & 0.51 & 0.23 & 0.17 & 0.10 & 0.85 & & & \\
$\tau^{2}$ & 0.02 & 0.00 & 0.07 & 0.00 & 0.06 & 0.30 & & \\
$p$ & 0.04 & 0.00 & 0.00 & 0.00 & 0.02 & 0.00 & 0.24 & \\
$m$ & 0.01 & 0.01 & 0.00 & 0.00 & 0.02 & 0.00 & 0.02 & 0.05 \\
\hline & Method & $k$ & $\bar{n}$ & $\rho$ & $\beta$ & $\tau^{2}$ & $p$ & $m$
\end{tabular}

moderators (i.e., $\beta$ ), which is also responsible for distinguishing conditions where the empty model was the true model from conditions where moderators were actually present, followed by the number of effect sizes $(k)$ and the sample size of the primary studies $(\bar{n})$ (in supplementary Figure S1, we provide a plot of the probabilities of identifying the true model as a function of the various design factors for each of the 40 methods). Furthermore, the size of the coefficient showed strong interactions with the selection method, the number of effect sizes, and the sample size of the primary studies.

Based on these findings, we constructed Figure 2.3, which shows the performance of six representative methods as a function of the coefficient size when $k$ is equal to $20,40,60$, and 80 . We included selection via univariate and full model testing as reference benchmarks and used the AICc criterion combined with the $l l_{R E M L r}$ function for each of the information-theoretic approaches (Figures S2 and S3 provided as part of the supplementary materials are analogous figures using the AIC and BIC criteria, but show very similar patterns).

When the true model was the empty model (i.e., $\beta=0$ ), we again see the superior performance of the information criteria methods compared to univariate or full model testing. While the latter two methods were able to identify the true model on average in about $70 \%$ of the cases, using multimodel inference and an RVI with a cutoff of 0.8 yielded essentially $100 \%$ correct identification rates regardless of $k$, closely followed by using the minimum AICc or an RVI with a cutoff of 0.5 for model selection. 

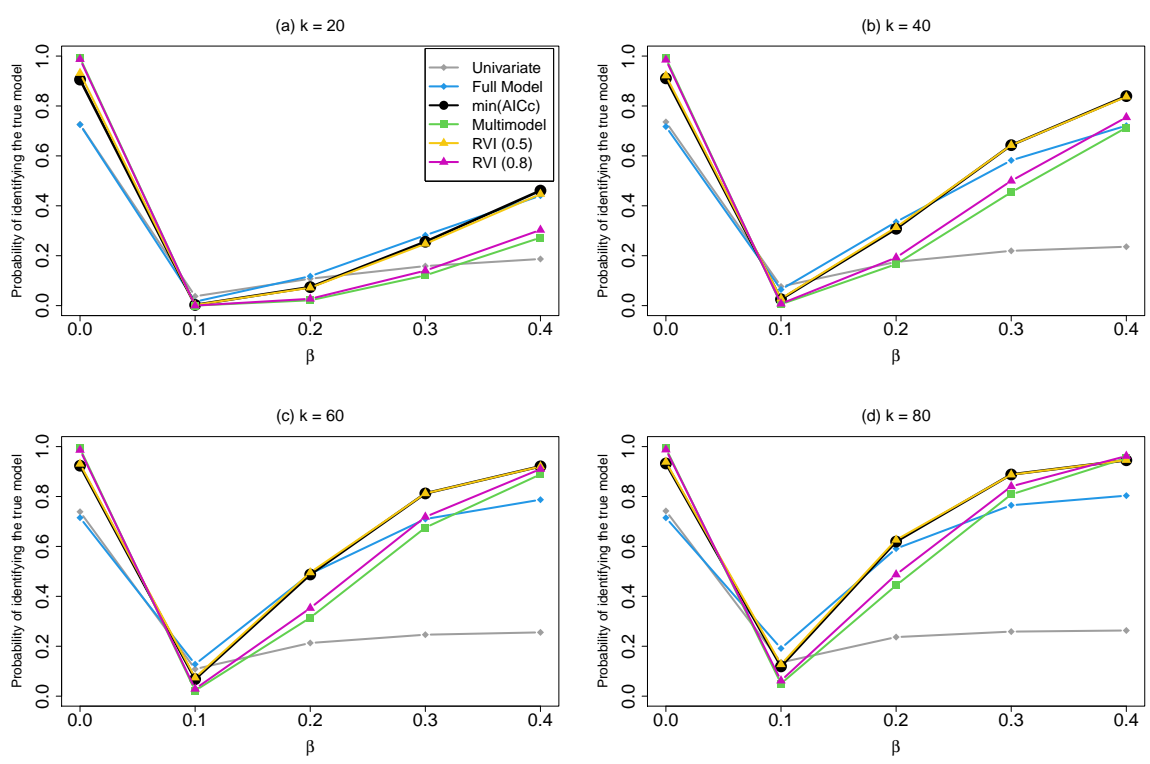

Figure 2.3: Probabilities of identifying the true model of 6 model selection methods as a function of $\beta$ and $k$. The methods shown are selection via univariate and full model testing and the information-theoretic approaches using the AICc criterion combined with the $l l_{R E M L r}$ function. Each line represents the probabilities of a model selection method averaged over the remaining factors.

When there is a weak association between the effect sizes and the moderators (i.e., $\beta=0.1$ ), all methods yielded very low probabilities (i.e., $\leq 0.10$ ), with little improvements as $k$ increased. Selection via full model testing may have a slight advantage over the remaining methods in this case especially when $k$ is large. On the other hand, while larger coefficients and values of $k$ led to increases in the probabilities for all methods (except for univariate testing, whose performance flattened out at around 20\%), selection via the minimum AICc or an RVI with a cutoff of 0.5 outperformed full model testing especially when $k$ and $\beta$ were large. In fact, while all information criteria methods converged to $100 \%$ rates with increases in $k$ and $\beta$, this does not appear to be the case for full model testing, which seems to reach a maximum identification rate around $80 \%$. Supplementary Figures S4 to S7 provide analogous figures to Figure 2.3, but separately for each value of $\bar{n}$, showing that this maximum 
is also reached even when the sample size of the primary studies increases.

\subsection{Discussion}

In this paper, we describe methods for model selection in meta-regression with particular emphasis on information-theoretic approaches. In doing so, we hope to introduce researchers unfamiliar with such methods to a new set of tools that may be useful in applied research. However, one should only consider applying novel methods if evidence suggests that there are actual advantages to using these approaches over more conventional methods for model selection, such as testing moderator variables one at a time in a series of meta-regression models (i.e., what we denote 'univariate testing') or testing moderator variables in a single meta-regression model (i.e., 'full model testing'). We therefore also conducted a simulation study to examine the performance of the various model selection methods for identifying the set of moderators that are actually related to the effect sizes.

Across a wide variety of conditions, the results show that the informationtheoretic approaches often outperform the more conventional methods. This was especially apparent for conditions where none of the moderators were truly related to the effect sizes (i.e., the 'empty model' was the true model), but could also be seen when there was a relationship between some of the moderators and the effect sizes. Only when the relationship was very weak was there a slight advantage to using a full model testing approach, although differences between methods were small and all methods had rather low probabilities of identifying the true model in such conditions.

Of all methods evaluated, univariate testing performed especially poorly even when the relationship between moderators and effect sizes was strong and the number of studies was large. This finding is not surprising, given that correlated moderator variables were simulated in many conditions. Univariate testing will then often find that 'false' moderators are significant simply because they happen to be correlated (i.e., confounded) with the true moderators (a phenomenon also known as omitted-variable bias). At the same time, when testing a true moderator without other relevant moderators included in the model, at least part of the heterogeneity that could have been accounted for by the other moderators will then be subsumed into the the random effects term used to model residual heterogeneity. This in turn will decrease the power to detect the true moderator. Hence, too many false and too few true moderators will be correctly identified when using univariate testing, leading 
to the poor performance of this model selection strategy. Although concerns about univariate testing of moderators have been raised before [e.g., 33], these concerns warrant repetition, given that univariate testing is still the dominant approach in meta-regression analyses [7].

Full model testing can circumvent these problems, at least if we assume that all potentially relevant moderators are included in the model (or that those omitted are not correlated with the ones included in the model). In that case, the correlation among the moderators is correctly taken into consideration, avoiding omitted-variable bias. Consequently, this model strategy also fared much better in the simulation study, but the results also indicate that the probability of identifying the true model did not converge to $100 \%$ even when both the number of studies and the size of the regression coefficient increased.

There is a simple explanation for this finding. Even when the power to detect the true moderators is $100 \%$ so that all true moderators will be correctly identified, each false moderator tested incurs a certain chance of committing a Type I error. The more such false moderators are tested, the higher the probability that at least one of them will turn out to be significant. Given the design of the simulation study, there were either $f=2,3,4$, or 5 false moderators in conditions where the number of true moderators was set to half of the number of moderators tested or there were either $f=2,4,6$, or 8 false moderators in conditions where the number of true moderators was fixed at 2 . The probability that at least one of the false moderators turns out significant is $1-(1-\alpha)^{f}$, which we can compute for each value of $f$ above (with $\alpha=.05$ ). Averaging the resulting values yields approximately 0.19 . Hence, we should see that the probability of correctly identifying the true model converges to $1-.19=.81$, which is exactly what Figure 2.3 suggests (see panel $(\mathrm{d})$ for $k=80$ and $\beta=0.4)$.

An obvious solution to this problem is to apply a multiple testing correction for each of the moderators tested in the context of the full model. Although this will also reduce power to detect true moderators, $100 \%$ power should be restored with sufficiently large $k$ and $\beta$. There will still be a small probability that at least one false moderator is selected and hence a perfect model identification probability cannot be achieved, but the discrepancy should be minor. However, we did not explore this strategy in the simulation study, as the use of multiple testing corrections in the context of meta-regression analyses is rare $[7]$.

In contrast, all methods based on information criteria achieved perfect or near perfect model identification probabilities when $k$ and $\beta$ increased. This also 
applies to the multimodel testing approach, which seems counterintuitive given that we did not use a multiple testing correction in the context of this strategy either. However, this model selection strategy goes beyond full model testing by also using model-averaged parameter estimates and standard errors for doing so. The way the model-averaged parameter estimates are computed - by assigning zero to the coefficient for a particular moderator variable in models where the moderator does not appear - will automatically lead to a shrinkage effect when models without a particular moderator receive large Akaike weights [10]. This effect can also be seen in the illustrative example (cf. Table 2.3) especially for the coefficients corresponding to the mycorrhizal fungus (FUN) and soil sterilization (STER) moderators, given that almost $70 \%$ of the total weight (i.e., $(1-\mathrm{RVI}) \times 100 \%)$ is placed on models in which these moderators do not appear. In this sense, multimodel testing shares some properties with other penalization techniques, such as ridge regression [34] and the lasso [35], although these methods have not been extended to the metaregression context.

Simply selecting a model based on the minimum of a particular information criterion or using the RVI values with a certain cutoff for variable selection works equally well as the multimodel testing approach when $k$ and $\beta$ are large. For all these strategies, an increasingly large weight will then be placed on the model that corresponds to the true model, leading to perfect model identification probabilities. In less favorable circumstances, the best method seems to depend on whether one favors a method that has better chances of detecting that none of the moderators are actually related to the effect sizes (in which case multimodel testing or using the RVIs with a cutoff of 0.8 appear preferable) or whether one would like to optimize one's chances that the method will correctly sort out which moderators are and which ones are not related to the effect sizes (in which case selection based on the minimum of an information criterion or using the RVIs with a cutoff of 0.5 would be the better choice).

An additional noteworthy finding of the simulation study is that model selection via information criteria computed based on the REML function appears to be a valid strategy. In principle, this finding goes against the common wisdom that REML functions (and hence information criteria) computed based on models with different fixed effects are not comparable [e.g., 12, 13]. The present findings are however in line with those of [14], who also presented results to the contrary, but in a different modeling context. However, when using information criteria based on REML estimation, one has to carefully consider how exactly the restricted likelihood will be computed, that is, whether the $\frac{1}{2} \ln \left|\boldsymbol{X}^{\prime} \boldsymbol{X}\right|$ term should be included in the likelihood function or not. While 
the findings are somewhat mixed and the right approach might depend on the strategy and information criterion used, the results clearly indicate that omission of the the term is important to obtain high probabilities of correct model identification when the empty model is actually the true model.

Regardless of the chosen strategy, a practical issue that frequently arises in meta-regression analyses is missing data. In particular, while some moderator variables are easy to extract (e.g., the publication year of a study) and will be essentially complete, missing data on other moderator variables is a common occurrence. A comparison of models containing different subsets of moderator variables will then be hampered by the fact that the models will be based on different subsets of the data, which makes their likelihoods and hence the information criteria incomparable. On the other hand, using only the set of studies with complete information on all moderator variables of interest will typically lead to a substantial reduction in the number of studies included in the analysis. Note that this will also automatically happen when using full model testing, as 'listwise deletion' is the default behavior in all software for meta-regression that we are aware of. In fact, we suspect that the prevalence of univariate testing may at least in part stem from the desire of authors to maximize the number of studies included in each meta-regression model. However, as the current results show, univariate testing cannot be recommended as a general model selection strategy. Alternatively, one could consider methods for imputing missing moderator values [36, 37], although very little work has examined the performance of such methods in the meta-analytic context so far [but see 38]. How to combine imputation methods with model selection strategies including the ones discussed in the present paper could therefore be the subject of future research.

We are fully aware of cautions in the literature against simply using all possible models as candidates for the true model instead of conducting an analysis based on a smaller set of candidate hypotheses and their corresponding models [e.g., 8, 10]. Even though we used this approach in the simulation study, this should be seen as an abstraction done for the purposes of simplifying the simulation study. Also, potential moderators should always be a priori specified [6] (ideally with a hypothesis why and how they might be related to the effect sizes) and hence the $p$ moderators examined in the simulation study could be considered to be a selection based on a larger number of potential moderators.

Furthermore, we note that the 'true model' was included in the set of candidate models in our simulation study, although this is unlikely to correspond to 
reality, which is more complex than any statistical model we might formulate $[8,10]$. Hence, in practice, the true model is not going to be part of the candidate set. However, this scenario is difficult to simulate and would require that we quantify to what extent each model approximates some true data generating mechanism. At the same time, by allowing for (residual) heterogeneity, random- and mixed-effects models in meta-analysis already include a term that is meant to capture any influences on the effect sizes that the model is unable to account for. Conditions where $\tau^{2}>0$ are therefore scenarios where even the true model from the candidate set could just be considered to be the best approximation to the actual data generating mechanism, however complex it may be.

In conclusion, the present article provides some initial evidence that conventional methods for model selection, such as univariate and full-model testing, may be outperformed by information-theoretic approaches. The latter are more often among the set of best methods across all of the conditions simulated and can have higher probabilities for identifying the true model under particular scenarios. We recommend that authors of meta-analyses involving meta-regression analyses consider the use of these methods, especially as an alternative to univariate testing. 
Chapter 2. Using information-theoretic approaches for model selection in meta-analysis

\section{References}

[1] H. Cooper, L. V. Hedges, and J. C. Valentine, eds. The handbook of research synthesis and meta-analysis. 2nd. New York: Russell Sage Foundation, 2009.

[2] C. S. Berkey et al. "A random-effects regression model for meta-analysis". In: Statistics in Medicine 14.4 (1995), pp. 395-411.

[3] H. C. van Houwelingen, L. R. Arends, and T. Stijnen. "Advanced methods in meta-analysis: Multivariate approach and meta-regression". In: Statistics in Medicine 21.4 (2002), pp. 589-624.

[4] S. W. Raudenbush. "Analyzing effect sizes: Random-effects models". In: The handbook of research synthesis and meta-analysis. Ed. by H. Cooper, L. V. Hedges, and J. C. Valentine. 2nd. New York: Russell Sage Foundation, 2009, pp. 295-315.

[5] S. G. Thompson and S. J. Sharp. "Explaining heterogeneity in metaanalysis: A comparison of methods". In: Statistics in Medicine 18.20 (1999), pp. 2693-2708.

[6] S. G. Thompson and J. P. T. Higgins. "How should meta-regression analyses be undertaken and interpreted?" In: Statistics in Medicine 21.11 (2002), pp. 1559-1573.

[7] E. Tipton, J. E. Pustejovsky, and H. Ahmadi. "Current practices in meta-regression in psychology, education, and medicine". In: Research Synthesis Methods 10.2 (2019), pp. 180-194.

[8] K. P. Burnham and D. R. Anderson. Model selection and multimodel inference: A practical information-theoretic approach. 2nd. New York: Springer, 2002.

[9] M. J. Whittingham et al. "Why do we still use stepwise modelling in ecology and behaviour?" In: Journal of Animal Ecology 75.5 (2006), pp. $1182-1189$.

[10] D. R. Anderson. Model based inference in the life sciences: A primer on evidence. 2nd. New York: Springer, 2007.

[11] C. Chatfield. "Model uncertainty, data mining and statistical inference". In: Journal of the Royal Statistical Society, Series A 158.3 (1995), pp. 419-466.

[12] J. C. Pinheiro and D. M. Bates. Mixed-effects models in $S$ and S-PLUS. New York: Springer, 2000. 
[13] G. Verbeke and G. Molenberghs. Linear mixed models for longitudinal data. New York: Springer, 2000.

[14] M. J. Gurka. "Selecting the best linear mixed model under REML". In: American Statistician 60.1 (2006), pp. 19-26.

[15] P. Shi and C.-L. Tsai. "Regression model selection: A residual likelihood approach". In: Journal of the Royal Statistical Society, Series B 64.2 (2002), pp. 237-252.

[16] G. C. Fernandez. Model selection in PROC MIXED: A user-friendly SAS macro application. Paper presented at the SAS Global Forum. Orlando, FL, Apr. 2007.

[17] M. Borenstein. "Effect sizes for continuous data". In: The handbook of research synthesis and meta-analysis. Ed. by H. Cooper, L. V. Hedges, and J. C. Valentine. 2nd. New York: Russell Sage Foundation, 2009, pp. 221-235.

[18] J. L. Fleiss and J. A. Berlin. "Effect sizes for dichotomous data". In: The handbook of research synthesis and meta-analysis. Ed. by H. Cooper, L. V. Hedges, and J. C. Valentine. 2nd. New York: Russell Sage Foundation, 2009, pp. 237-253.

[19] W. Viechtbauer. "Bias and efficiency of meta-analytic variance estimators in the random-effects model". In: Journal of Educational and Behavioral Statistics 30.3 (2005), pp. 261-293.

[20] J. Nocedal and S. J. Wright. Numerical optimization. 2nd. New York: Springer, 2006.

[21] W. Viechtbauer et al. "A comparison of procedures to test for moderators in mixed-effects meta-regression models". In: Psychological Methods 20.3 (2015), pp. 360-374.

[22] H. Akaike. "A new look at the statistical model identification". In: IEEE Transactions on Automatic Control 19.6 (1974), pp. 716-723.

[23] G. Schwarz. "Estimating the dimension of a model". In: Annals of Statistics 6.2 (1978), pp. 461-464.

[24] C. M. Hurvich and C.-L. Tsai. "Regression and time series model selection in small samples". In: Biometrika 76.2 (1989), pp. 297-307.

[25] C. M. Hurvich and C.-L. Tsai. "Bias of the corrected AIC criterion for underfitted regression and time series models". In: Biometrika 78.3 (1991), pp. 499-509. 
Chapter 2. Using information-theoretic approaches for model selection in meta-analysis

[26] D. A. Harville. "Maximum likelihood approaches to variance component estimation and to related problems". In: Journal of the American Statistical Association 72.358 (1977), pp. 320-338.

[27] H. D. Patterson and R. Thompson. "Recovery of inter-block information when block sizes are unequal". In: Biometrika 58.3 (1971), pp. 545-554.

[28] P. M. Lukacs, K. P. Burnham, and D. R. Anderson. "Model selection bias and Freedman's paradox". In: Annals of the Institute of Statistical Mathematics 62.1 (2010), pp. 117-125.

[29] V. B. Chaudhary et al. "MycoDB, a global database of plant response to mycorrhizal fungi". In: Scientific Data 3 (2016), p. 160028.

[30] L. V. Hedges, J. Gurevitch, and P. S. Curtis. "The meta-analysis of response ratios in experimental ecology". In: Ecology 80.4 (1999), pp. 11501156 .

[31] Julian PT Higgins et al. "Measuring inconsistency in meta-analyses". In: Bmj 327.7414 (2003), pp. 557-560.

[32] R Core Team. R: A Language and Environment for Statistical Computing. R Foundation for Statistical Computing. Vienna, Austria, 2020.

[33] M. W. Lipsey. "Those confounded moderators in meta-analysis: Good, bad, and ugly". In: The Annals of the American Academy of Political and Social Science 587 (2003), pp. 69-81.

[34] A. E. Hoerl and R. W. Kennard. "Ridge regression: Biased estimation for nonorthogonal problems". In: Technometrics 12.1 (1970), pp. 55-67.

[35] R. Tibshirani. "Regression shrinkage and selection via the lasso". In: Journal of the Royal Statistical Society, Series B 58.1 (1996), pp. 267288.

[36] T. D. Pigott. "Methods for handling missing data in research synthesis". In: The handbook of research synthesis. Ed. by H. Cooper and L. V. Hedges. New York: Russell Sage Foundation, 1994, pp. 163-176.

[37] T. D. Pigott. "Missing predictors in models of effect size". In: Evaluation and the Health Professions 24.3 (2001), pp. 277-307.

[38] E Hance Ellington et al. "Using multiple imputation to estimate missing data in meta-regression". In: Methods in Ecology and Evolution 6.2 (2015), pp. 153-163. 


\section{Supplementary Materials}

1

10

$\sqrt{+\square} \square$

要: $\square$

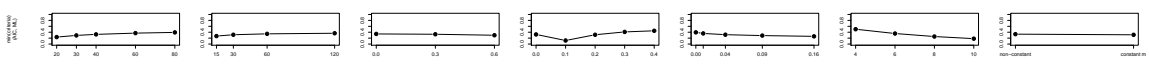

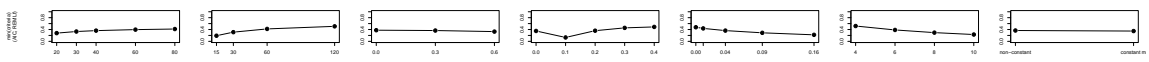

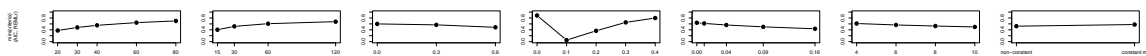

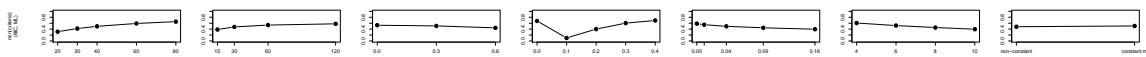

II

$\| \stackrel{+\square !}{\square \square}$

$1 \longdiv { \square ! } \square$

$\sqrt{\square ! \square} \square \square$

$1 1 \longdiv { \square \square } + \square$

最:

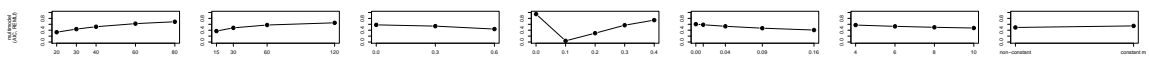

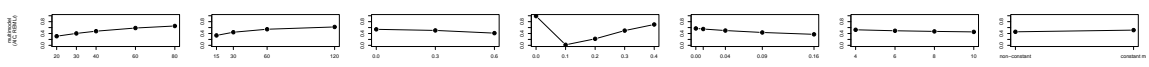

居吅

展吅

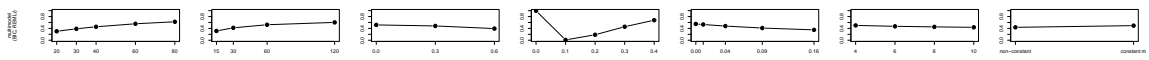

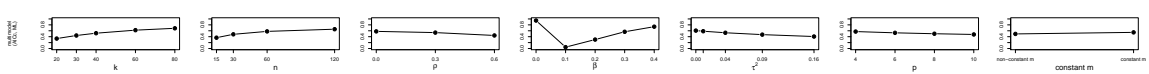




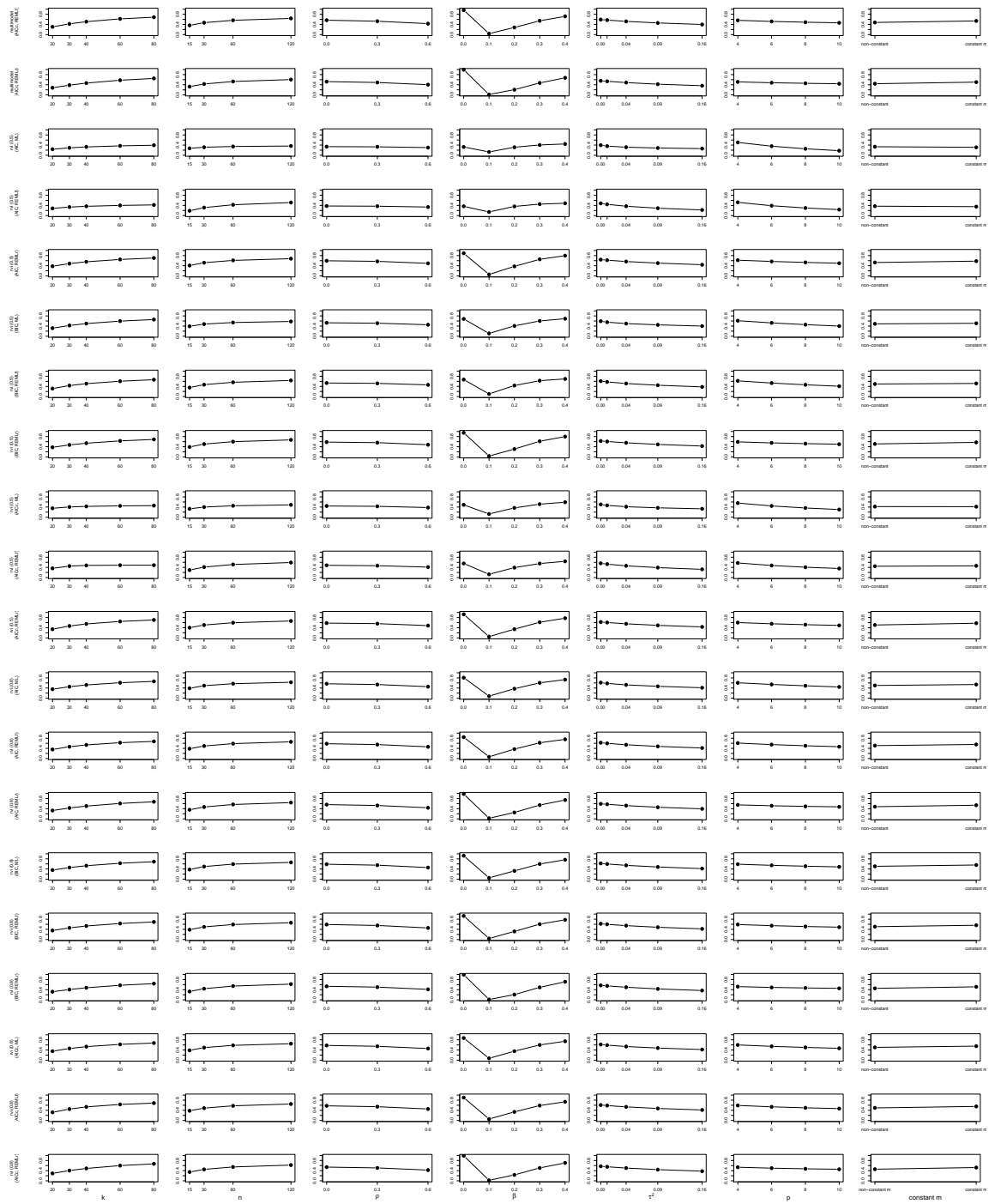

Figure S1: Probabilities of identifying the true model for each of the 40 model selection methods as functions of the factors used in the simulation. The values in the panels represent the probabilities averaged over the remaining factors. 

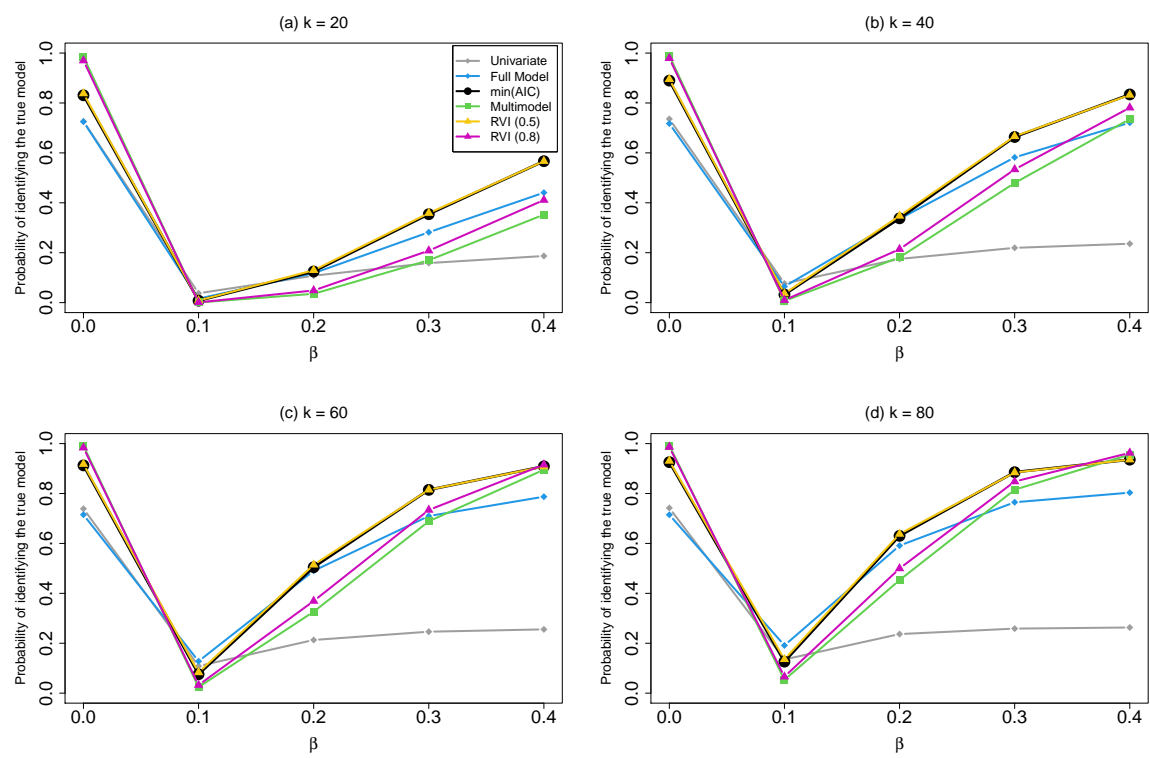

Figure S2: Probabilities of identifying the true model of 6 model selection methods as a function of $\beta$ and $k$. The methods shown are selection via univariate and full model testing and the information-theoretic approaches using the AIC criterion combined with the $l l_{R E M L r}$ function. Each line represents the probabilities of a model selection method averaged over the remaining factors. 
Chapter 2. Using information-theoretic approaches for model selection in meta-analysis
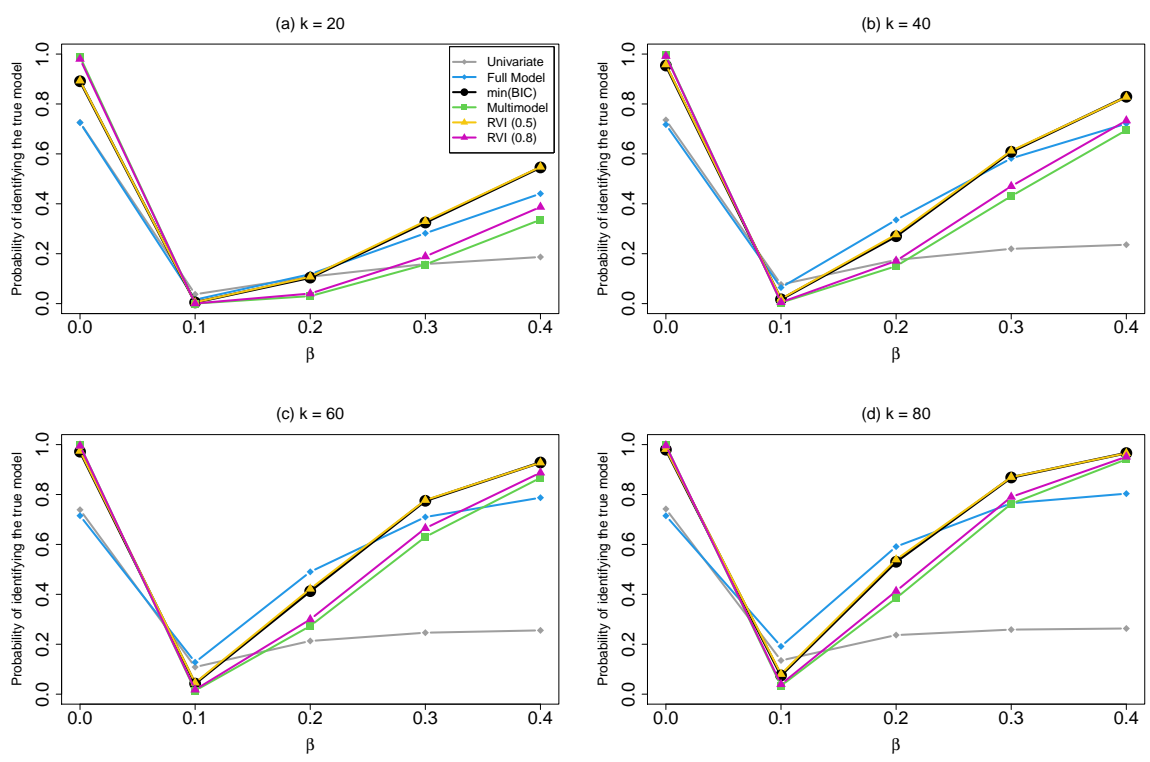

Figure S3: Probabilities of identifying the true model of 6 model selection methods as a function of $\beta$ and $k$. The methods shown are selection via univariate and full model testing and the information-theoretic approaches using the BIC criterion combined with the $l l_{R E M L r}$ function. Each line represents the probabilities of a model selection method averaged over the remaining factors. 

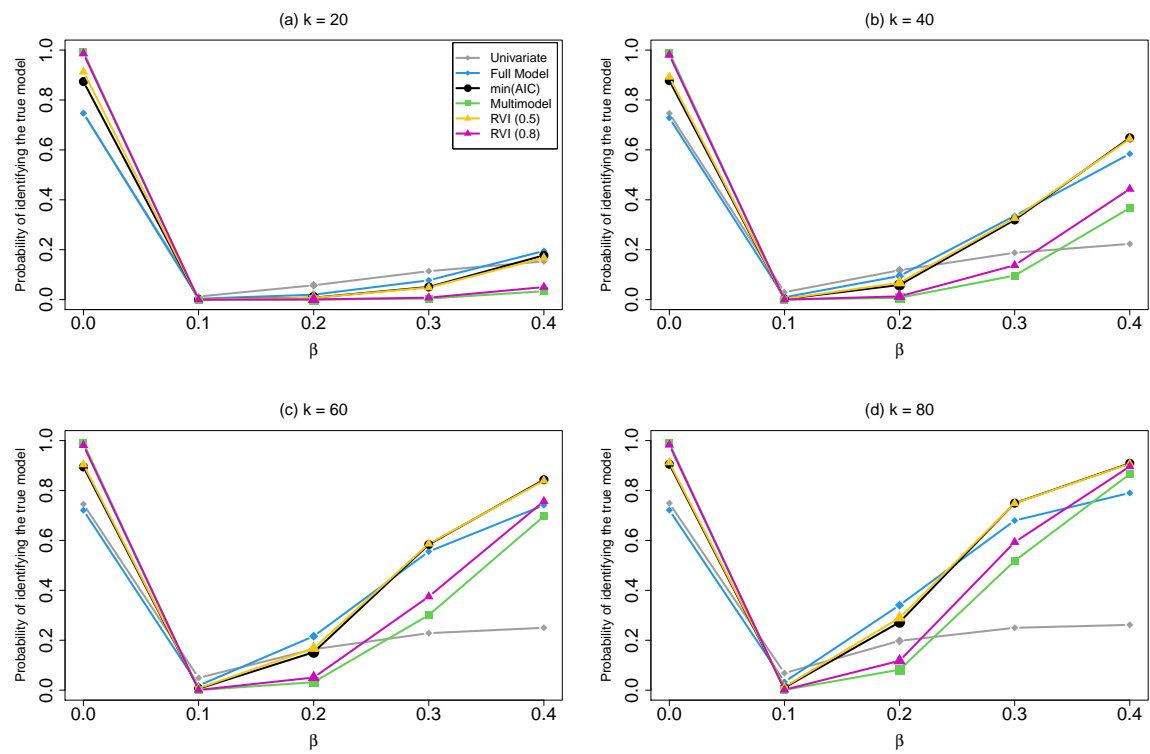

Figure S4: The probabilities of identifying the true model of 6 model selection methods as functions of two factors, $k$ and $\beta$, used in the simulation when $n=15$. The methods shown are selection via univariate and full model testing and the information-theoretic approaches using the AICc criterion combined with the $l l_{R E M L r}$ function. Each line represents the probabilities of a model selection method averaged over the remaining factors. 
Chapter 2. Using information-theoretic approaches for model selection in meta-analysis
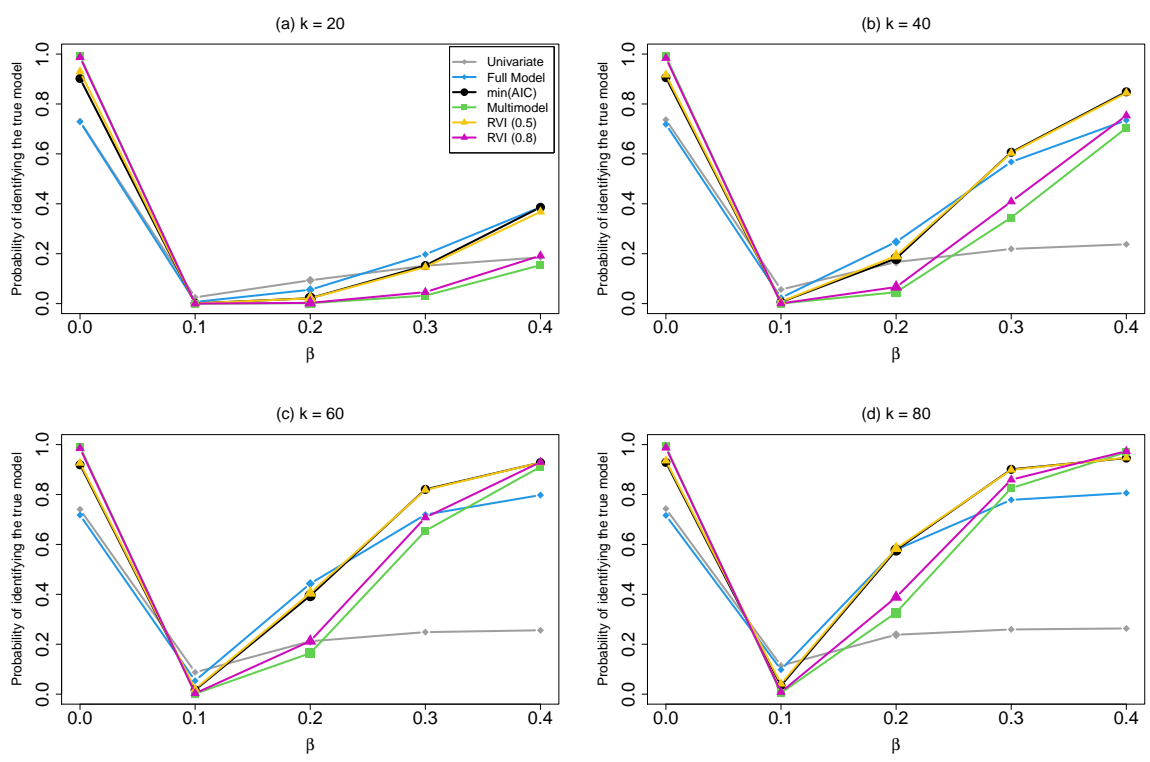

Figure S5: The probabilities of identifying the true model of 6 model selection methods as functions of two factors, $k$ and $\beta$, used in the simulation when $n=30$. The methods shown are selection via univariate and full model testing and the information-theoretic approaches using the AICc criterion combined with the $l l_{R E M L r}$ function. Each line represents the probabilities of a model selection method averaged over the remaining factors. 

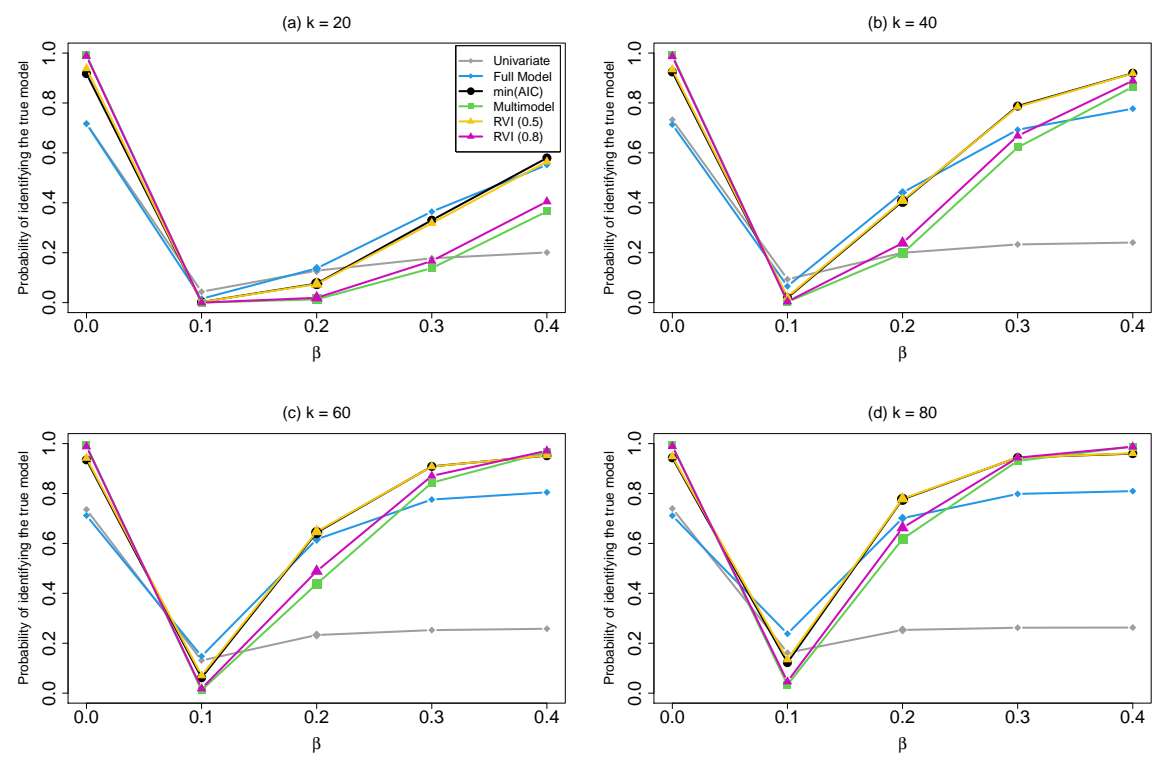

Figure S6: The probabilities of identifying the true model of 6 model selection methods as functions of two factors, $k$ and $\beta$, used in the simulation when $n=60$. The methods shown are selection via univariate and full model testing and the information-theoretic approaches using the AICc criterion combined with the $l l_{R E M L r}$ function. Each line represents the probabilities of a model selection method averaged over the remaining factors. 
Chapter 2. Using information-theoretic approaches for model selection in meta-analysis
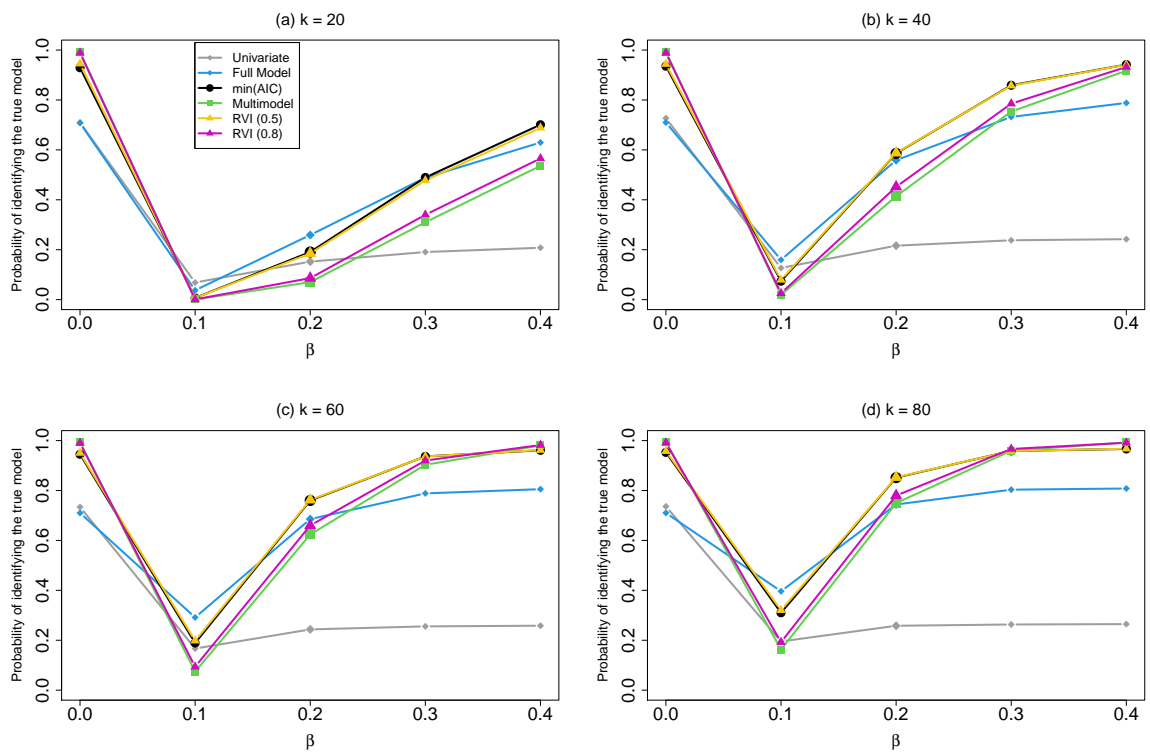

Figure S7: The probabilities of identifying the true model of 6 model selection methods as functions of two factors, $k$ and $\beta$, used in the simulation when $n=120$. The methods shown are selection via univariate and full model testing and the information-theoretic approaches using the AICc criterion combined with the $l l_{R E M L r}$ function. Each line represents the probabilities of a model selection method averaged over the remaining factors. 


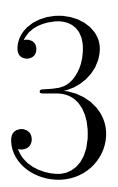

Phylogenetic multilevel meta-analysis: A simulation study on the importance of modeling the phylogeny

Ozan Cinar, Shinichi Nakagawa, and Wolfgang Viechtbauer. "Phylogenetic multilevel meta-analysis: A simulation study on the importance of modeling the phylogeny". In: ((Submitted to) Ecology Letters). 


\section{Abstract}

Meta-analyses in ecology and evolution typically include multiple estimates from the same study and based on multiple species. The resulting dependencies in the data can be addressed by using a phylogenetic multilevel meta-analysis model. However, the complexity of the model poses challenges for accurately estimating model parameter. We therefore carried out a simulation study to investigate the performance of models with different degrees of complexities. While the overall mean was estimated with little to no bias irrespective of the model, only the model that accounted for the multilevel structure and that incorporates both a non-phylogenetic and a phylogenetic variance component provided confidence intervals with approximately nominal coverage rates. We therefore suggest that meta-analysts in ecology and evolution use the phylogenetic multilevel meta-analysis model as the de facto standard when analyzing multi-species datasets. 


\subsection{Introduction}

Meta-analysis encompasses an array of methods for synthesizing information from primary studies and evaluating the consistency of their results $[1,2,3$, 4]. Although these methods have been mostly developed in the medical and social sciences $[3,5,6]$, ecologists and evolutionary biologists have successfully adopted these techniques for conducting research syntheses in their respective fields $[7,8,9]$. However, meta-analyses in ecology and evolution typically have several features that require special attention so that trustworthy evidence can be obtained.

To start, meta-analyses in these fields often incorporate data from multiple species which share an evolutionary history, known as phylogeny $[10,11,12]$. As a result, the samples (and the effect sizes obtained from these samples) are not independent which violates the independence assumption underlying conventional meta-analytic models. For example, the standard fixed- and randomeffects models [see 2, 13], often used for ecological meta-analyses [14], assume independence among the effect sizes and therefore do not account for the phylogeny $[12,15]$. This issue was first addressed by two meta-analytic methods proposed by Adams [16] and Lajeunesse [17] which incorporate phylogeny into the fixed- and random-effects models, respectively.

Chamberlain et al. [12] empirically investigated how the inclusion of phylogeny affects the estimate of the overall mean based on data from 30 meta-analyses in ecology and evolution. They showed that the estimate of the overall mean did not change considerably in most of the meta-analyses, especially when using a random-effects model for the analysis. However, a substantial portion of the meta-analyses, which reported significant results before, produced non-significant results when the phylogeny was incorporated into the model. Therefore, including phylogeny might be an important factor to reduce Type I error rates and to obtain an accurate reflection of the uncertainty of metaanalytic estimates.

Although Chamberlain et al. [12] is the most extensive study to date to examine the effects of phylogeny in meta-analysis, there are two potential limitations. First, their work was based on available meta-analyses. To investigate the issue of phylogeny more broadly, we require a simulation study to explore a wider parameter space and under controlled conditions. Second, Chamberlain et al. [12] did not address the fact that ecological and evolutionary studies usually report multiple effect sizes per study, which leads to yet another source of non-independence in a meta-analysis $[14,15]$. Although past and current 
meta-analyses have sometimes avoided this issue by selecting a single effect size from each study or by collapsing multiple effect sizes into one, these procedures can lead to a severe loss of information [14].

As an alternative, Hadfield and Nakagawa [18] proposed a mixed-effects model that accounts for the multilevel structure in the data via a study-level random effect (i.e., multiple effect sizes per study are nested within this random effect). In the same model, they include two additional random effects to estimate the non-phylogenetic and the phylogenetic variance. This way, among-species variance can be decomposed into two components, the one resulting from species similarities due to evolutionary history and the other from species similarities due to shared ecology and other factors [19]. Although the model by Hadfield and Nakagawa [18] addresses two major statistical issues in ecological and evolutionary meta-analyses, the complexity of the model poses certain challenges.

Partitioning the among-species variance into its two components is a challenging endeavor, because both components are modeled using random effects at the species level, with the only difference that the phylogenetic component assumes that the random effects for different species are correlated according to a phylogenetic correlation matrix - which in turn is derived from a phylogenetic tree that is constructed based on the similarities and differences of species in terms of their (usually) genetic (but sometimes also physical) characteristics [20]. This raises concerns about the identifiability of the corresponding variance components and potential bias in their estimates, issues that have also been raised outside the meta-analytic context when analyzing the data of primary studies that include multiple species [21].

Even if the parameters can be estimated unbiasedly, the complexity of the model poses a threat to the convergence of the optimization algorithms [22]. Accordingly, Nakagawa and Santos [14] suggested that model fitting may only be feasible with larger datasets, which would limit the applicability of the model in practice. To avoid these problems, some ecological and evolutionary meta-analyses were carried out using a more parsimonious model by removing the non-phylogenetic random effect and have therefore dealt with the species variance only via the phylogenetic component [e.g., 23, 24]. However, the consequences of doing so, and the performance of the more complex model, has yet to be evaluated in a simulation study.

Here we aim to investigate the performance of models for conducting a phylogenetic multilevel meta-analysis in a comprehensive simulation study. We simulate studies that report multiple effect sizes and use several models that 
vary in their complexity, starting from a simple model (including only a random effect at the effect-size level) to the most complex model which incorporates a study-level random effect and two among-species random effects. Further, we generate specific conditions to test the performance of the most complex model when the phylogenetic relationships are weak and to examine the consequences of removing the non-phylogenetic component. Before we fully explain our simulation design, we first introduce the different meta-analytic models in further detail.

\subsection{Meta-Analytic Models}

To conduct a meta-analysis, the phenomenon of interest (e.g., the size of a treatment effect or the strength of the association between two variables) needs to be quantified in terms of an effect size estimate for each study to be included in the analysis. Note that we use the term study broadly here, as a single study may contribute multiple effect size estimates (e.g., for multiple species, subgroups, different treatments), but for the moment we assume that each study contributes a single estimate to the meta-analysis.

The specific effect size measure to be used in a meta-analysis depends on the phenomenon of interest and the information reported in the primary studies [14]. For example, raw or standardized mean differences and response ratios [25] are typically used to quantify group differences or treatment effects based on quantitative variables, correlation coefficients (or Fisher r-to-z transformed values thereof) reflect the (linear) relationship between two variables, while (log-transformed) odds/risk ratios and risk differences (calculated from $2 \times 2$ contingency tables) indicate group differences (e.g., treated vs. untreated, exposed vs. non-exposed) with respect to dichotomous dependent variables (e.g., cured vs. not cured, diseased vs. not diseased). For all of these measures, we can also derive an equation that allows us to compute the sampling variances of the effect size estimates, that is, the variability in each estimate that would be expected under repeated sampling of new study units under identical circumstances [3, 26, 27].

Regardless of the specific measure used in a meta-analysis, let $y_{i}$ denote the effect size estimate for the $i$ th study (with $i=1, \ldots, N_{\text {studies }}$ ) and $v_{i}$ the corresponding sampling variance. The most basic model that can be considered for synthesizing the estimates is the fixed-effects model, which is given by

$$
y_{i}=\mu+e_{i},
$$




$$
\mathbf{e} \sim N(\mathbf{0}, \mathbf{V}),
$$

where $\mu$ is the overall mean, $e_{i}$ is the sampling error for the $i$ th study, e is a $1 \times N_{\text {studies }}$ column vector with the $e_{i}$ values (which are assumed to be normally distributed with mean 0 and variance $\left.v_{i}\right), \mathbf{0}$ is a column vector of zeros, and $\mathbf{V}$ is an $N_{\text {studies }} \times N_{\text {studies }}$ matrix with the $v_{i}$ values along the diagonal.

The fixed-effects model assumes that the studies included in a meta-analysis share a single common true effect. This assumption, however, is rarely met in multi-population and multi-species meta-analyses of ecology and evolution studies $[11,28]$. The random-effects model addresses this potential 'heterogeneity' among the true effects by adding a random effect corresponding to each estimate and is given by

$$
\begin{aligned}
& y_{i}=\mu+u_{i}+e_{i} \\
& \mathbf{u} \sim N\left(\mathbf{0}, \sigma_{u}^{2} \mathbf{I}_{u}\right),
\end{aligned}
$$

where $u_{i}$ is the random effect corresponding to the $i$ th estimate, $\mathbf{u}$ is a $1 \times$ $N_{\text {studies }}$ column vector with the $u_{i}$ values (which are assumed to be normally distributed with mean 0 and variance $\sigma_{u}^{2}$ ), and $\mathbf{I}_{u}$ is an $N_{\text {studies }} \times N_{\text {studies }}$ identity matrix.

Although the models above are suitable for conducting a meta-analysis in many circumstances, they do not account for the multilevel structure that arises when (at least some of) the studies provide multiple effect size estimates (e.g., when the same experiment was conducted several times under varying circumstances within the same study) and they do not account for phylogenetic dependence (when studies are conducted with multiple species that differ in similarity due to differences in their shared evolutionary history).

To address the first issue, we can use a multilevel meta-analytic model [14, 29] which includes a random effect at the effect size level (as in model 3.3), but which now captures variability in the true effects within studies, and a random effect at the study level, which captures between-study variability. Let $y_{i j}$ denote the $j$ th effect in the $i$ th study (with $j=1, \ldots, N_{i}$, where $N_{i}$ is the number of effect sizes reported in the $i$ th study), $v_{i j}$ the corresponding sampling variance, and let $N_{\text {total }}=\sum_{i=1}^{N_{\text {studies }}} N_{i}$ denote the total number of effects. The model is then given by

$$
y_{i j}=\mu+u_{i j}+s_{i}+e_{i j}
$$




$$
\mathbf{s} \sim N\left(\mathbf{0}, \sigma_{s}^{2} \mathbf{I}_{s}\right)
$$

where $u_{i j}$ is a random effect corresponding to the $j$ th effect size in the $i$ th study, $s_{i}$ is a random effect at the study level, $\mathbf{u}$ is now a $1 \times N_{\text {total }}$ column vector with the $u_{i j}$ values, $\mathbf{s}$ is a $1 \times N_{\text {studies }}$ column vector with the $s_{i}$ values (which are assumed to be normally distributed with mean 0 and variance $\sigma_{s}^{2}$ ), and $\mathbf{I}_{u}$ and $\mathbf{I}_{s}$ are $N_{\text {total }} \times N_{\text {total }}$ and $N_{\text {studies }} \times N_{\text {studies }}$ identity matrices, respectively. Finally, e is now defined as a $1 \times N_{\text {total }}$ column vector with the $e_{i j}$ values and $\mathbf{V}$ is the corresponding (diagonal) variance-covariance matrix with dimensions $N_{\text {total }} \times N_{\text {total }}$.

To deal with multiple species (i.e., when the effect size estimates were computed based on a set of $N_{\text {species }}$ different species), we will need an additional index. Hence, let $y_{i j k}$ denote the $j$ th effect in the $i$ th study as before, but now let $k=1, \ldots, N_{\text {species }}$ be the index that indicates for which species a particular effect size estimate was computed. Model 3.5 can then be extended to account for species-level variability as follows:

$$
\begin{gathered}
y_{i j k}=\mu+u_{i j}+s_{i}+n_{k}+e_{i j}, \\
\mathbf{n} \sim N\left(\mathbf{0}, \sigma_{n}^{2} \mathbf{I}_{n}\right),
\end{gathered}
$$

where $n_{k}$ is a species-specific random effect, $\mathbf{n}$ is a $1 \times N_{\text {species }}$ column vector with the $n_{k}$ values (which are assumed to be normally distributed with mean 0 and between-species variance $\sigma_{n}^{2}$ ), and $\mathbf{I}_{n}$ has dimensions $N_{\text {species }} \times N_{\text {species }}$. Note that $n_{k}$ is a crossed random effect [e.g., 30] and not nested within studies and we do not need subscript $k$ for $u_{i j}$ or $e_{i j}$ since subscripts $i$ and $j$ are sufficient to uniquely identify each effect size estimate in the dataset.

Model 3.7, however, does not account for phylogeny. For this, we can further extend the model by including an additional set of species-level random effects [18], but instead of assuming independence for different species (as for the $n_{k}$ values), we allow these random effects to be correlated according to a phylogenetic correlation matrix, which in turn is derived from a phylogenetic tree based on some model of evolution (such as Brownian motion) prior to the analysis [e.g., 17, 20, 31, 32]. The model is then given by

$$
\begin{gathered}
y_{i j k}=\mu+u_{i j}+s_{i}+n_{k}+p_{k}+e_{i j}, \\
\mathbf{p} \sim N\left(\mathbf{0}, \sigma_{p}^{2} \mathbf{A}\right),
\end{gathered}
$$

where $p_{k}$ denotes the phylogenetic random effect for the $k$ th species, $\mathbf{p}$ is a $1 \times N_{\text {species }}$ column vector with the $p_{k}$ values (which are assumed to follow 
a multivariate normal distribution with mean 0 and variance-covariance matrix $\sigma_{p}^{2} \mathbf{A}$, where $\mathbf{A}$ is the $N_{\text {species }} \times N_{\text {species }}$ phylogenetic correlation matrix). Hence, the model includes non-phylogenetic species-level random effects (i.e., the $n_{k}$ values) to account for heterogeneity in the effects sizes due to differences between species unrelated to phylogeny (e.g., the influence of differences in the environments they live in) and the phylogenetic random effects (i.e., the $p_{k}$ values) that capture dependencies in the effect sizes according to the similarities between species due to phylogenetic relatedness.

A concern with model 3.9 arises when the phylogenetic relationships are weak. In that case $\mathbf{A}$ starts to resemble $\mathbf{I}_{n}$, and hence $\sigma_{p}^{2}$ and $\sigma_{n}^{2}$ are confounded and may not be uniquely identifiable. This in turn may lead to bias in the estimates of the variance components. This concern, or the complexity of model 3.9 in general, has led some researchers to adopt a more parsimonious model in their ecological and evolutionary meta-analyses where the non-phylogenetic variance component is removed. This leads to the model

$$
y_{i j k}=\mu+u_{i j}+s_{i}+p_{k}+e_{i j},
$$

where all terms are as explained before. Whether such a simplified version is an adequate substitute for model 3.9 is currently unknown.

All of the models described above can be fitted within a Bayesian or likelihood framework [18]. For the latter, the metafor package [33] for $\mathrm{R}$ [34] is particularly attractive as it is freely available and was written specifically for the purposes of conducting meta-analyses. Maximum likelihood (ML) or restricted maximum likelihood (REML) estimation can be used for model fitting (the latter usually being the preferred choice; [35]), which will provide estimates of the variance components included in a particular model, the estimate of $\mu$ (i.e., $\hat{\mu})$, and its corresponding standard error (i.e., $\mathrm{SE}[\hat{\mu}])$. Likelihood ratio tests and profile likelihood confidence intervals provide inferences for the variance components. A Wald-type $95 \%$ confidence interval for $\mu$ can be obtained with $\hat{\mu} \pm 1.96 \mathrm{SE}[\hat{\mu}]$.

\subsection{Simulation}

We explored the consequences of using the various models described in the previous section based on a simulation study. 


\begin{tabular}{ccccccccc}
$N_{\text {studies }}$ & $N_{\text {species }}$ & $\sigma_{u}^{2}$ & $\sigma_{s}^{2}$ & $\sigma_{n}^{2}$ & $\sigma_{p}^{2}$ & $\alpha$ & Conditions & True model \\
\hline 20 & 40 & $0,0.05,0.30$ & 0 & 0 & 0 & 1 & 3 & Model 3 \\
20 & 40 & $0.05,0.30$ & $0.05,0.30$ & 0 & 0 & 1 & Model 5 \\
20 & 40 & $0.05,0.30$ & $0.05,0.30$ & $0.05,0.30$ & 0 & $0.5,1,2$ & 24 & Model 7 \\
20 & 40 & $0.05,0.30$ & $0.05,0.30$ & $0.05,0.30$ & $0.05,0.30$ & $0.5,1,2$ & 48 & Model 9 \\
50 & 100 & $0,0.05,0.30$ & 0 & 0 & 0 & 1 & 3 & Model 3 \\
50 & 100 & $0.05,0.30$ & $0.05,0.30$ & 0 & 0 & 1 & 4 & Model 5 \\
50 & 100 & $0.05,0.30$ & $0.05,0.30$ & $0.05,0.30$ & 0 & $0.5,1,2$ & 24 & Model 7 \\
50 & 100 & $0.05,0.30$ & $0.05,0.30$ & $0.05,0.30$ & $0.05,0.30$ & $0.5,1,2$ & 48 & Model 9
\end{tabular}

Table 3.1: Overview of the conditions examined in the simulation study. The first two columns show the number of studies and species, respectively. The next four columns indicate the true values of the variance components. The $\alpha$ column represent the power parameter. All values were crossed within a particular row of the table. The last two columns respectively represent the number of conditions generated in each row and the model that corresponds to the true data generating mechanism for the conditions in a particular row.

\subsubsection{Simulation Setup}

In our setup, the primary studies considered in a meta-analysis could provide one or multiple effect size estimates for one or multiple species. We set $\left(N_{\text {studies }}, N_{\text {species }}\right)$ either to $(20,40)$ or $(50,100)$ to examine the difference between a smaller versus larger meta-analysis. Furthermore, we set $\sigma_{u}^{2}, \sigma_{s}^{2}, \sigma_{n}^{2}$, and $\sigma_{p}^{2}$ to either $0,0.05$, or 0.3 (plus an additional parameter $\alpha$ to be defined below to either $0.5,1$, or 2 ) to define a particular condition within the simulation study. Table 3.1 provides an overview of the 158 conditions that were studied in this manner. Note that we used a 'conditional factorization' of the four variance components to keep the number of conditions manageable and to generate scenarios where one of the models described in Eqs. 3.3, 3.5, 3.7, and 3.9 corresponds to the true data generating mechanism. Within a particular condition, the following steps were repeated 1000 times.

First, the number of effect sizes provided by the studies (i.e., the $N_{i}$ values) were simulated from a right-skewed distribution, as typically observed in practice. For this, we generated $N_{\text {studies }}$ random values from a $\operatorname{Beta}(1.5,3)$ distribution, which were then multiplied by 39 , rounded to the closest integer, and increased by 1 . Therefore, the number of estimates per study could vary between 1 and 40 (with a mean, median, and mode of approximately 14, 13, and 9 , respectively).

In the next step, we simulated the species indices (i.e., the $k$ values) by generating $N_{\text {total }}$ random values from a $\operatorname{Beta}(2,2)$ distribution, which were multiplied by $N_{\text {species }}-1$, rounded to the closest integer, and then increased by 1 . Hence, 
(a) $\alpha=0.5$

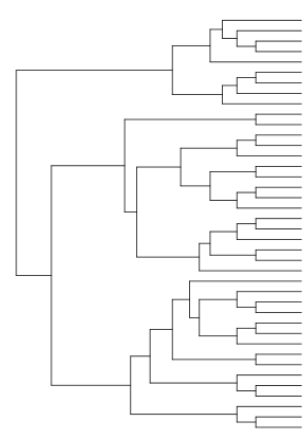

(b) $\alpha=1$

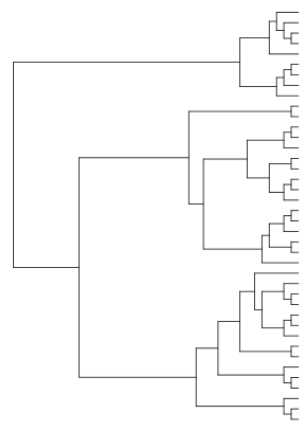

(c) $\alpha=2$

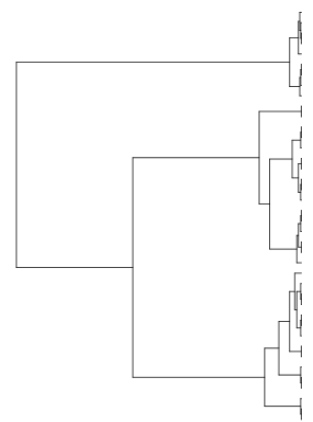

Figure 3.1: An example of a simulated phylogenetic tree for 40 species modified with different values of the power parameter $\alpha$ (i.e., $0.5,1$, and 2 ).

the number of times that the various species were studied followed a symmetric unimodal distribution (with mean/median/mode equal to $\left.\left(N_{\text {species }}+1\right) / 2\right)$. In order to guarantee that all species appear at least once in the meta-analysis, a randomly chosen $N_{\text {species }}$ random numbers generated this way were replaced with the integers from 1 to $N_{\text {species }}$.

Next, we generated a phylogenetic tree for the species using the rtree() function from the $\mathrm{R}$ package ape [36], which uses a simple recursive random splitting algorithm to simulate a phylogeny [21]. The branch lengths were then computed using the compute.brlen() function from the same package based on the method by Grafen [37], using the power parameter $\alpha$ to essentially adjusts the 'height' of branch lengths at the tips of the phylogenetic tree, leading to phylogenetic relationships that are generally stronger when branches are shorter at the tips or weaker when branches are longer at the tips. Figure 3.1 shows an example of such a simulated tree for 40 species modified by different $\alpha$ values. Finally, the correlation matrix that represents the phylogenetic relationships (denoted by $\mathbf{A}$ in Eq. 3.10) was calculated from the tree by using the vcv () function based on a Brownian model of evolution (i.e., $\mathbf{A}_{k, k^{\prime}}=1-b_{k, k^{\prime}}$, where $b_{k, k^{\prime}}$ is the branch length for a pair of species to their most recent common ancestor).

We then generated the four sets of random effects, corresponding to the variance components $\sigma_{u}^{2}, \sigma_{s}^{2}, \sigma_{n}^{2}$, and $\sigma_{p}^{2}$, either as independent draws from normal 
distributions for the first three components or from a multivariate normal distribution for the last one. In conditions where a particular variance component is equal to 0 , the corresponding random effects are then just a series of $0 \mathrm{~s}$ of the appropriate length. To complete the data generating step, the sampling variances (i.e., the $v_{i j}$ values) were simulated from a right-skewed $\operatorname{Beta}(2,20)$ distribution (and hence had a value of .091 on average) which were then used to generate the $N_{\text {total }}$ sampling errors from a normal distribution with mean 0 and variance $v_{i j}$. We then summed up the random effects and sampling errors as shown in Eqs. 3.9, setting $\mu=0$ without loss of generality.

After generating the data, we fitted the four models shown in equations 3.3, 3.5, 3.7, and 3.9, using REML estimation as implemented in the rma.mv() function from the metafor package. For model 3.3, we simply treated each estimate as a separate study (one can also think of this as model 3.5 without the addition of the study-level random effect). For each model, we then saved the estimate of $\mu$, the variance component estimates, the bounds of the $95 \%$ Wald-type confidence interval for $\mu$, and the model fitting time. In case any one of the four models did not converge within a particular iteration (with the default settings of the rma.mv() function), the iteration was discarded and a new iteration was run to guarantee that a 1000 successful model fits were available for all four models.

After the 1000 iterations, we computed the mean of the $\hat{\mu}$ values for each model, the mean of the variance component estimates, the proportion of iterations where 0 was included in the confidence interval (i.e., the empirical coverage rate), the mean confidence interval width, the convergence rate, and the mean model fitting time. The simulation was run on a workstation with an Intel Xeon E5-2630v4 processor utilizing 15 cores in parallel. Completion time for the simulation was approximately 7 days (roughly 2520 core hours in total).

We generated two other sets of conditions to investigate specific questions. In the first set, we examined conditions where the phylogenetic relationships could also be weaker than in the main scenarios to test the performance of model 3.9 under such conditions. These conditions were generated by setting $\alpha$ to $(0.1,0.2,0.3,0.4,0.5,1,2)$ when $\left(N_{\text {studies }}, N_{\text {species }}\right)=(50,100)$, the estimate- and study-level variance components were both large $(0.3)$, and the levels of the remaining variance components were factorized with values of 0.05 and 0.3 (for a total of 28 different conditions). In the second set, we compared the performance of model 3.9 and the simplified model 3.11 (that leaves out the non-phylogenetic species-level variance component). For this, we set $\left(N_{\text {studies }}, N_{\text {species }}\right)=(50,100), \sigma_{u}^{2}=0.05, \sigma_{s}^{2}=0.05$, and $\alpha=1$, and then 
generated different conditions by factorizing different values of only $\sigma_{n}^{2}$ and $\sigma_{p}^{2}$, where the former was set to values from 0 to 0.3 with increments of 0.05 , whereas the latter was set to either $0,0.05$, or 0.3 (for a total of 21 different conditions).

\subsubsection{Results}

Figure $3.2 \mathrm{a}$ displays boxplots based on the mean $\hat{\mu}$ value for each of the four models across the 158 conditions, separated by which model was the true data generating mechanism. Generally, the means were clustered tightly around 0 , indicating little to no bias in $\hat{\mu}$, although in a small set of conditions there was some slight positive bias in the estimates of the overall mean. These conditions were characterized by non-zero values for all four variance components (i.e., when model 3.9 was the true model $),\left(N_{\text {studies }}, N_{\text {species }}\right)=(20,40)$, a weak phylogenetic relationship $(\alpha=0.5)$, and a large phylogenetic variance $\left(\sigma_{p}^{2}=\right.$ $0.3)$.

In contrast to the results for the overall mean, the coverage rates of the $95 \%$ confidence interval for $\mu$ differed markedly across models (Figure 3.2b). For conditions where model 3.3 was the true data generating mechanism, all models achieved coverage rates close to or slightly above the nominal $95 \%$ confidence level regardless of the specific conditions. As the other variance components were introduced into the data, however, the coverage rates of models that did not account for these additional sources of variability started to decrease, at times severely so. Only model 3.9 was able to achieve rates close to the nominal level across the majority of conditions, although the rates also fell somewhat below the nominal level for certain conditions when all variance components were larger than zero.

Given that estimates of $\mu$ were relatively unbiased for all models, the closer to nominal coverage rates of model 3.9 would be expected to be a consequence of wider confidence interval (that consequently have a better chance of capturing the true value of $\mu$ ). Figure $3.2 \mathrm{c}$ confirms this, showing the mean confidence interval widths for the various models across the various conditions. However, what is particularly noteworthy is that the use of model 3.9 under conditions where actually a simpler model is the true data generating mechanism only leads to a relatively minor increase in the mean interval width.

Figure 3.3 displays the bias in the variance component estimates of model 3.9 under the 28 different conditions generated by varying $\alpha, \sigma_{n}^{2}$, and $\sigma_{p}^{2}$ (while holding $\sigma_{u}^{2}$ and $\sigma_{s}^{2}$ constant at 0.3 ). The results show no bias in the estimates 

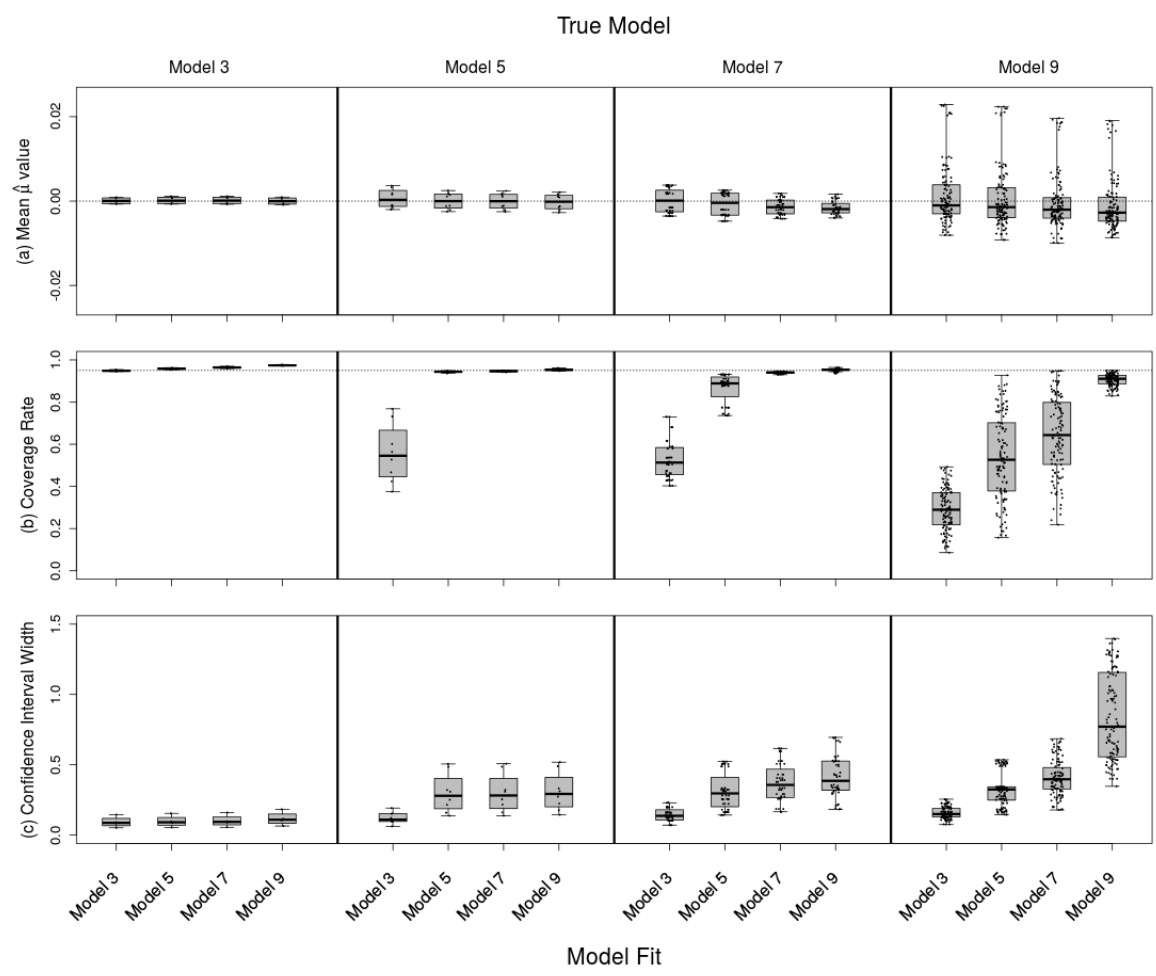

Figure 3.2: Boxplots based on the (a) mean $\hat{\mu}$ value, (b) coverage rates of the $95 \%$ confidence interval for $\mu$, and (c) mean confidence interval widths for each of the four models across the 158 conditions, separated by which model was the true data generating mechanism.

of $\sigma_{u}^{2}$ and $\sigma_{s}^{2}$. Furthermore, the model is able to estimate $\sigma_{n}^{2}$ and $\sigma_{p}^{2}$ with little to no bias, except when the strength of the phylogenetic relationships decreased. As expected, under such conditions, the model struggles to provide unbiased estimates of the non-phylogenetic and phylogenetic species-level variance components.

Figure 3.4a shows the coverage rates of the confidence interval for $\mu$ for models 3.9 and 3.11 as the size of the non-phylogenetic species-level variance component (i.e., $\sigma_{n}^{2}$ ) was systematically increased. While model 3.9 provided rates 

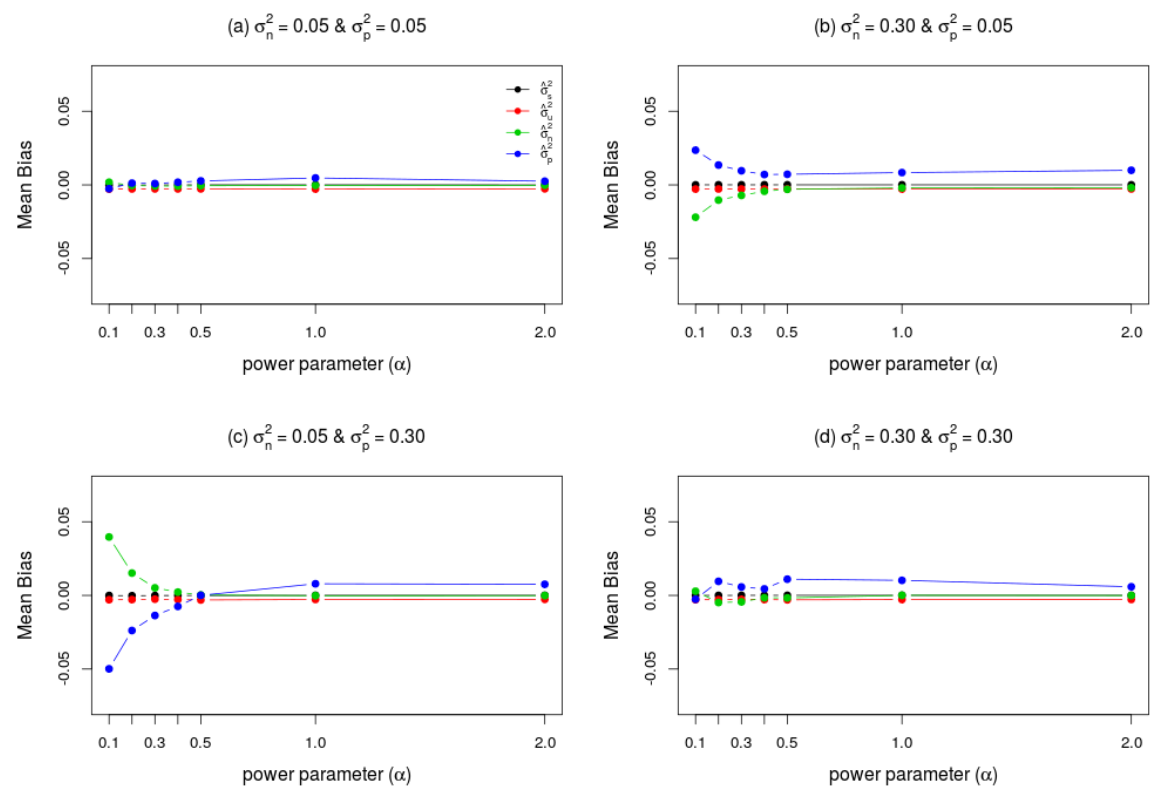

Figure 3.3: Mean bias of the variance component estimates of model 3.9 under different combinations of the power parameter $(\alpha)$ and the non-phylogenetic and phylogenetic variance components $\left(\sigma_{n}^{2}\right.$ and $\sigma_{p}^{2}$, respectively).

close to or somewhat below the nominal level, the rates for model 3.11 were often equal to $100 \%$ and hence the confidence interval for the overall mean tended to be too wide (i.e., was overly conservative). Furthermore, Figure 3.4b demonstrates that the bias in the phylogenetic variance component of model 3.11 inflated rapidly as the true value of $\sigma_{n}^{2}$ increased (the value of $\sigma_{p}^{2}$ had no noteworthy influence on the size of the bias and hence we averaged these results over the three possible values of $\sigma_{p}^{2}$ ). In contrast, model 3.9 estimated these two variance components essentially without bias under these scenarios.

Model fitting times differed between the various models (Table 3.2), with model 3.9 requiring the most amount of time on average, regardless of the true data generating mechanism. The most challenging conditions for the more complex models were those scenarios where model 3.3 corresponded to the true data generating mechanism. In this case, a single fit of model 3.9 took around 26 seconds on average when $\left(N_{\text {studies }}, N_{\text {species }}\right)=(50,100)$. In these conditions, 

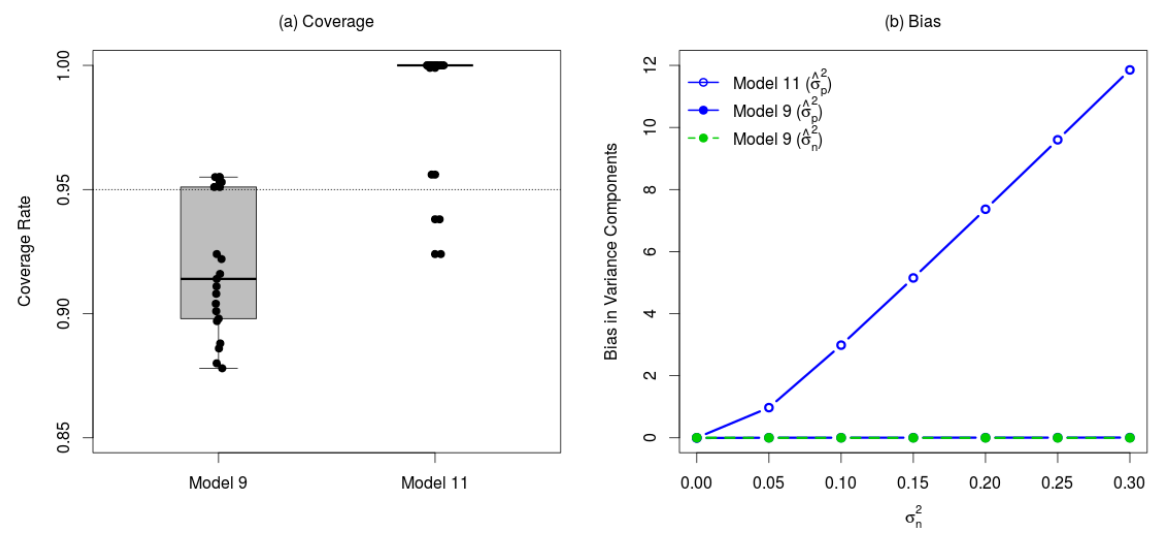

Figure 3.4: Comparison of models 3.9 and 3.11 as the size of the non-phylogenetic species-level variance component (i.e., $\sigma_{n}^{2}$ ) was systematically increased. (a) Coverage rates of the $95 \%$ confidence intervals for $\mu$, (b) bias in the non-phylogenetic and phylogenetic variance components.

\begin{tabular}{ccccc} 
& \multicolumn{4}{c}{ (a) $\left(N_{\text {studies }}, N_{\text {Species }}\right)=(20,40)$} \\
& \multicolumn{4}{c}{ True Model } \\
\cline { 2 - 5 } Model Fit & Model 3 & Model 5 & Model 7 & Model 9 \\
\hline \multirow{2}{*}{ Model 3 } & 0.939 & 0.668 & 0.700 & 0.700 \\
& $(100.00 \%)$ & $(100.00 \%)$ & $(100.00 \%)$ & $(100.00 \%)$ \\
Model 5 & 2.653 & 1.104 & 1.151 & 1.162 \\
& $(99.81 \%)$ & $(100.00 \%)$ & $(100.00 \%)$ & $(100.00 \%)$ \\
Model 7 & 2.484 & 1.876 & 0.868 & 0.858 \\
& $(97.53 \%)$ & $(100.00 \%)$ & $(100.00 \%)$ & $(100.00 \%)$ \\
Model 9 & 3.316 & 3.053 & 2.288 & 1.463 \\
& $(96.56 \%)$ & $(99.73 \%)$ & $(99.99 \%)$ & $(99.99 \%)$ \\
\hline
\end{tabular}

\begin{tabular}{ccccc} 
& \multicolumn{4}{c}{ (b) $\left(N_{\text {studies }}, N_{\text {Species }}\right)=(50,100)$} \\
True Model \\
\cline { 2 - 5 } Model Fit & Model 3 & Model 5 & Model 7 & Model 9 \\
\hline \multirow{2}{*}{ Model 3 } & 1.589 & 1.313 & 1.307 & 1.294 \\
& $(100.00 \%)$ & $(100.00 \%)$ & $(100.00 \%)$ & $(100.00 \%)$ \\
Model 5 & 3.934 & 1.986 & 1.999 & 1.959 \\
& $(99.78 \%)$ & $(100.00 \%)$ & $(100.00 \%)$ & $(100.00 \%)$ \\
Model 7 & 19.823 & 14.752 & 7.364 & 7.393 \\
& $(96.86 \%)$ & $(100.00 \%)$ & $(100.00 \%)$ & $(100.00 \%)$ \\
Model 9 & 25.980 & 23.540 & 18.641 & 11.005 \\
& $(95.63 \%)$ & $(99.60 \%)$ & $(100.00 \%)$ & $(100.00 \%)$ \\
\hline
\end{tabular}

Table 3.2: Average model fitting times in seconds and convergence rates (in parentheses) of all models under the different data generating mechanisms.

convergence rates were also the lowest, although even model 3.9 then converged in approximately $96 \%$ of the iterations.

\subsection{Discussion}

Meta-analyses in the fields of ecology and evolution typically need to address the fact that multiple effect size estimates can be extracted from at least some of the studies and that the estimates are based on various species that are related to each other due to their shared evolutionary history. In this paper, 
we investigated the performance of the phylogenetic multilevel meta-analytic model proposed by Hadfield and Nakagawa [18] and Nakagawa and Santos [14] that captures these intricacies along with some simpler models. Despite the concerns we raised in the introduction, the model can successfully estimate the overall mean and its uncertainty. It also provides approximately unbiased estimates of all variance components, including the non-phylogenetic and phylogenetic species-level variances, as long as there are at least moderately strong phylogenetic relationships among the species. In addition, despite its complexity, the model does not appear to suffer from convergence problems and model fitting does not require excessive computational times.

\subsubsection{Estimating the Overall Mean and its Uncertainty}

Not only the phylogenetic multilevel meta-analytic model, but also the simpler models that leave out certain variance components provide essentially unbiased estimates of the overall mean, regardless of the nature of the true model that underlies the data (Figure 3.2a). However, the uncertainty in the overall mean will only be estimated accurately when the fitted model includes the variance components that do contribute to the heterogeneity and dependencies among the underlying true effects. Fitting underspecified models typically led to severe undercoverage of the confidence interval for the overall mean and hence anticonservative inferences. In fact, subtracting the coverage rates shown in Figure $3.2 \mathrm{~b}$ from 1 yields the Type I error rates for the test of the overall mean, which could go as high as $91 \%$ when using a simple random-effects model that ignores the multilevel structure and the species-level variance components.

These findings are in line with those by Chamberlain et al. [12], who demonstrated, based on 30 published meta-analyses, that the inclusion of phylogeny into a random-effects model usually only led to minor changes in the pooled effect size, but had a more substantial impact on the statistical significance of the finding (turning significant findings into non-significant ones in the majority of cases where changes occurred).

Our findings can also be used to alleviate concerns with using the phylogenetic multilevel meta-analytic model when it is actually an overspecified model (i.e., when the actual data generating mechanism is simpler). In those cases, the mean confidence interval width of the model was just barely wider than that of the simpler models, indicating little to no loss in efficiency by fitting an overly complex model (Figure 3.2c). The superfluous variance components then converge towards 0 (or close to it), which appears to be slightly more 
challenging for the optimization algorithm, leading to longer model fitting times and occasional convergence problems, but not to any worrisome degree (Table 3.2). Moreover, in practice, for any particular dataset, convergence problems can typically be resolved by selecting a different optimizer or making changes to the settings for the optimization routine, so the convergence rates as given only apply to the default settings.

At the same time, we should point out that the coverage rate of the model did fall slightly below the nominal $95 \%$ level in the majority of conditions when all variance components were in fact non-zero (see Figure 3.2b, rightmost panel). This undercoverage stems from using an overly simple Wald-type confidence interval using critical values based on a standard normal distribution that ignores the uncertainty in the estimates of the variance components (especially in the study and the two species-level components when $N_{\text {studies }}$ and $N_{\text {species }}$ are low). A similar issue, but for a simpler model with only between- and withinstudy variance components (i.e., model 3.5 in our simulation) was also recently pointed out by Song et al. [38]. Improved methods based on the t-distribution, with various approximations for the degrees of freedom, have been proposed and studied extensively in the context of the standard random-effects model [e.g., 39] and mixed-effects models in general [e.g., 40], but these methods have not been generalized to the present context. As a simple approximation, using the smaller of $N_{\text {studies }}-1$ and $N_{\text {species }}-1$ as the degrees of freedom for a confidence interval based on a t-distribution is likely to bring the coverage rate quite close to the nominal rate in the majority of conditions.

\subsubsection{Including and Testing the Phylogenetic Effect}

Phylogenies play a central role in the context of phylogenetic comparative studies $[32,41,42]$. An important step in such studies is testing the significance of the 'phylogenetic signal' in some trait of interest. This test is often performed through a statistic such as $\lambda$ [43] or $K$ [41]. Although model 3.9 does not parameterize the phylogenetic effect in this manner, one can derive information from its output that shows its relationship to the $\lambda$ statistic. In particular, Pagel's $\lambda$ is a multiplicative factor that is applied to the off-diagonal values of the correlation matrix that represents the phylogenetic relationships (i.e., the A matrix). For example, the variance-covariance matrix for three species would be given by

$$
\sigma^{2}\left[\begin{array}{ccc}
1 & \lambda a_{12} & \lambda a_{13} \\
& 1 & \lambda a_{23} \\
& & 1
\end{array}\right]
$$


while the decomposition of the species-level heterogeneity in model 3.9 implies the variance-covariance matrix

$$
\begin{aligned}
& \sigma_{n}^{2}\left[\begin{array}{ccc}
1 & & \\
& 1 & \\
& & 1
\end{array}\right]+\sigma_{p}^{2}\left[\begin{array}{ccc}
1 & a_{12} & a_{13} \\
& 1 & a_{23} \\
& & 1
\end{array}\right] \\
& \quad=\left(\sigma_{n}^{2}+\sigma_{p}^{2}\right)\left[\begin{array}{ccc}
1 & \left(\frac{\sigma_{p}^{2}}{\sigma_{n}^{2}+\sigma_{p}^{2}}\right) a_{12} & \left(\frac{\sigma_{p}^{2}}{\sigma_{n}^{2}+\sigma_{p}^{2}}\right) a_{13} \\
& 1 & \left(\frac{\sigma_{p}^{2}}{\sigma_{n}^{2}+\sigma_{p}^{2}}\right) a_{23} \\
& &
\end{array}\right]
\end{aligned}
$$

and hence $\sigma^{2}=\sigma_{n}^{2}+\sigma_{p}^{2}$ and $\lambda=\sigma_{p}^{2} /\left(\sigma_{n}^{2}+\sigma_{p}^{2}\right)$ [see also 19, 32]. Hence, $\sigma_{p}^{2} /\left(\sigma_{n}^{2}+\sigma_{p}^{2}\right)$ indicates the degree of phylogenetic signal in the overall variance sourced from the species. A likelihood ratio test of $H_{0}: \sigma_{p}^{2}=0$ can be easily performed by comparing $X^{2}=-2\left(l_{7}-l l_{9}\right)$ against a chi-squared distribution with one degree of freedom, where $l l_{7}$ and $l l_{9}$ are the (restricted) log likelihoods of models 3.7 and 3.9 , respectively.

\subsubsection{Estimating the Non-Phylogenetic and Phylogenetic Variance Components}

Given the informative nature of these two variance components, it is essential to estimate their true values accurately. We found that model 3.9 was usually able to estimate these variance components unbiasedly, but should note that the model struggles to separate the non-phylogenetic and phylogenetic species effects when phylogenetic relationships are weak. In essence, the two sources of variability then start to collapse into one, with a total variance of $\sigma_{n}^{2}+\sigma_{p}^{2}$. This total variance is then distributed in approximately equal parts into the two estimates, which explains the apparent low bias when (coincidentally) $\sigma_{n}^{2}=\sigma_{p}^{2}$ (Figure 3.3a and d). However, when $\sigma_{n}^{2} \neq \sigma_{p}^{2}$, the bias in the two estimates becomes quite apparent (Figure $3.3 \mathrm{~b}$ and $\mathrm{c}$ ). Therefore, we would caution against the use of model 3.9 when phylogenetic relationships are weak. As a rough guideline, for $\alpha=0.5$, the mean correlation in the $\mathbf{A}$ matrix (excluding the diagonal) is around 0.2 and hence a lower mean correlation would call into question the trustworthiness of the estimates of $\sigma_{n}^{2}$ and $\sigma_{p}^{2}$.

Some meta-analyses in ecology and evolution have used model 3.11 to reduce model complexity [e.g., 23, 24]. Our results indicate that this approach cannot be recommended. As we increased the value of $\sigma_{n}^{2}$ in the actual data, the bias in the phylogenetic variance component inflated massively as a result of leaving 
the non-phylogenetic variance component out of the model (Figure 3.4b). As a result, the relevance of the phylogeny could be greatly overestimated. In addition, the confidence interval for the overall mean then becomes extremely conservative with coverage rates at or very close to $100 \%$. This in turn implies a loss of efficiency for estimating the size of the overall mean and a loss of power for testing $H_{0}: \mu=0$.

\subsubsection{Caveats and Conclusions}

For the simulation study, we used a 'generic' effect size measure, that is, we directly simulated the sampling errors from a normal distribution and treated the sampling variances (i.e., the $v_{i j}$ values) as exactly known. These conditions only apply asymptotically to measures typically used in practice (e.g., standardized mean differences, response ratios, r-to-z transformed correlation coefficients, log-transformed risk/odds ratios). The present results therefore reflect the performance of the various models under idealized conditions (i.e., assuming that the sample sizes of the individual studies are sufficiently large, such that the sampling distributions of the estimates are indeed approximately normal and that any inaccuracies in the estimated sampling variances are negligible). The advantage of using a generic measure is that we were able to identify problems that are inherent to certain models and not (potentially) a consequence of violations to the model assumptions. On the other hand, it remains to be determined how well the phylogenetic multilevel model performs when the effect sizes are generated based on the exact distributional assumptions underlying specific measures.

Hence, at least for the moment, the present results suggest that model 3.9 is the most appropriate tool for conducting a multi-species meta-analysis in ecology and evolution. For the vast majority of conditions examined, it provides approximately unbiased estimates of the variance components and the overall mean and a confidence interval for the latter that has a close to nominal coverage rate. Therefore, we recommend that meta-analysts in ecology and evolution use the phylogenetic multilevel model as the de facto standard when analyzing multi-species datasets. 
Chapter 3. Phylogenetic multilevel meta-analysis: A simulation study on the importance of modeling the phylogeny

\section{References}

[1] Gene V Glass. "Primary, secondary, and meta-analysis of research". In: Educational researcher 5.10 (1976), pp. 3-8.

[2] Larry Hedges and Ingram Olkin. Statistical models for meta-analysis. New York: Academic Press, 1985.

[3] H. Cooper, L. V. Hedges, and J. C. Valentine, eds. The handbook of research synthesis and meta-analysis. 2nd. New York: Russell Sage Foundation, 2009.

[4] Alistair M Senior et al. "Heterogeneity in ecological and evolutionary meta-analyses: its magnitude and implications". In: Ecology 97.12 (2016), pp. 3293-3299.

[5] Matthias Egger, George Davey Smith, and Keith O'Rourke. "Introduction: rationale, potentials, and promise of systematic reviews". In: Systematic reviews in health care: meta-analysis in context (2001), pp. 119.

[6] Alexander J Sutton and Julian PT Higgins. "Recent developments in meta-analysis". In: Statistics in medicine 27.5 (2008), pp. 625-650.

[7] Jessica Gurevitch, Peter S Curtis, and Michael H Jones. "Meta-analysis in ecology". In: Advances in ecological research (2001).

[8] Julia Koricheva, Jessica Gurevitch, and Kerrie Mengersen. Handbook of meta-analysis in ecology and evolution. Princeton University Press, 2013.

[9] Jessica Gurevitch et al. "Meta-analysis and the science of research synthesis". In: Nature 555.7695 (2018), p. 175.

[10] Göran Arnqvist and David Wooster. "Meta-analysis: synthesizing research findings in ecology and evolution". In: Trends in Ecology 8 Evolution 10.6 (1995), pp. 236-240.

[11] Jessica Gurevitch and Larry V Hedges. "Statistical issues in ecological meta-analyses". In: Ecology 80.4 (1999), pp. 1142-1149.

[12] S. A. Chamberlain et al. "Does phylogeny matter? Assessing the impact of phylogenetic information in ecological meta-analysis". In: Ecology Letters 15.6 (2012), pp. 627-36.

[13] Larry V Hedges and Jack L Vevea. "Fixed-and random-effects models in meta-analysis." In: Psychological methods 3.4 (1998), p. 486.

[14] Shinichi Nakagawa and Eduardo SA Santos. "Methodological issues and advances in biological meta-analysis". In: Evolutionary Ecology 26.5 (2012), pp. 1253-1274. 
[15] Daniel WA Noble et al. "Nonindependence and sensitivity analyses in ecological and evolutionary meta-analyses". In: Molecular Ecology 26.9 (2017), pp. 2410-2425.

[16] Dean C. Adams. "Phylogenetic meta-analysis". In: Evolution 62.3 (Mar. 2008), pp. 567-572. DOI: 10.1111/j.1558-5646.2007.00314.x.

[17] Marc J Lajeunesse. "Meta-analysis and the comparative phylogenetic method". In: The American Naturalist 174.3 (2009), pp. 369-381.

[18] Jarrod D Hadfield and Shinichi Nakagawa. "General quantitative genetic methods for comparative biology: phylogenies, taxonomies and multitrait models for continuous and categorical characters". In: Journal of evolutionary biology 23.3 (2010), pp. 494-508.

[19] Michael Lynch. "Methods for the analysis of comparative data in evolutionary biology". In: Evolution 45.5 (1991), pp. 1065-1080.

[20] J. Felsenstein. Inferring phylogenies. 2nd. Sunderland, MA: Sinauer Associates, 2004.

[21] Emmanuel Paradis. Analysis of phylogenetics and evolution with R. 2nd. New York: Springer, 2012.

[22] Douglas Bates et al. "Parsimonious mixed models". In: arXiv preprint arXiv:1506.04967 (2015).

[23] László Zsolt Garamszegi, Gábor Markó, and Gábor Herczeg. "A meta-analysis of correlated behaviours with implications for behavioural syndromes: mean effect size, publication bias, phylogenetic effects and the role of mediator variables". In: Evolutionary Ecology 26.5 (2012), pp. $1213-1235$.

[24] Fhionna R Moore, David M Shuker, and Liam Dougherty. "Stress and sexual signaling: a systematic review and meta-analysis". In: Behavioral ecology 27.2 (2015), pp. 363-371.

[25] L. V. Hedges, J. Gurevitch, and P. S. Curtis. "The meta-analysis of response ratios in experimental ecology". In: Ecology 80.4 (1999), pp. 11501156 .

[26] Shinichi Nakagawa and Innes C Cuthill. "Effect size, confidence interval and statistical significance: a practical guide for biologists". In: Biological reviews 82.4 (2007), pp. 591-605.

[27] Michael Borenstein et al. Introduction to meta-analysis. John Wiley \& Sons, 2011. 
Chapter 3. Phylogenetic multilevel meta-analysis: A simulation study on the importance of modeling the phylogeny

[28] Julian PT Higgins, Simon G Thompson, and David J Spiegelhalter. "A re-evaluation of random-effects meta-analysis". In: Journal of the Royal Statistical Society: Series A (Statistics in Society) 172.1 (2009), pp. 137159.

[29] S. Konstantopoulos. "Fixed effects and variance components estimation in three-level meta-analysis". In: Research Synthesis Methods 2.1 (2011), pp. $61-76$.

[30] B. Fernández-Castilla et al. "A demonstration and evaluation of the use of cross-classified random-effects models for meta-analysis". In: Behavior Research Methods 51.3 (2019), pp. 1286-1304.

[31] Joseph Felsenstein. "Phylogenies and the comparative method". In: The American Naturalist 125.1 (1985), pp. 1-15.

[32] Rob P Freckleton, Paul H Harvey, and Mark Pagel. "Phylogenetic analysis and comparative data: a test and review of evidence". In: The American Naturalist 160.6 (2002), pp. 712-726.

[33] Wolfgang Viechtbauer. "Conducting meta-analyses in $\mathrm{R}$ with the metafor package". In: Journal of Statistical Software 36.3 (2010), pp. 1-48.

[34] R Core Team. R: A Language and Environment for Statistical Computing. R Foundation for Statistical Computing. Vienna, Austria, 2020.

[35] H. D. Patterson and R. Thompson. "Recovery of inter-block information when block sizes are unequal". In: Biometrika 58.3 (1971), pp. 545-554.

[36] Emmanuel Paradis and Klaus Schliep. "ape 5.0: An environment for modern phylogenetics and evolutionary analyses in R". In: Bioinformatics 35 (2019), pp. 526-528.

[37] Alan Grafen. "The phylogenetic regression". In: Phil. Trans. R. Soc. Lond. B 326.1233 (1989), pp. 119-157.

[38] Chao Song et al. "An assessment of statistical methods for non-independent data in ecological meta-analyses". In: Ecology ("in press"), e03184.

[39] J. Sanchez-Meca and F. Marin-Martinez. "Confidence intervals for the overall effect size in random-effects meta-analysis". In: Psychological Methods 13.1 (2008), pp. 31-48.

[40] Steven G Luke. "Evaluating significance in linear mixed-effects models in R". In: Behavior Research Methods 49.4 (2017), pp. 1494-1502. 
[41] Simon P Blomberg, Theodore Garland Jr, and Anthony R Ives. "Testing for phylogenetic signal in comparative data: behavioral traits are more labile". In: Evolution 57.4 (2003), pp. 717-745.

[42] Anthony R Ives, Peter E Midford, and Theodore Garland Jr. "Withinspecies variation and measurement error in phylogenetic comparative methods". In: Systematic Biology 56.2 (2007), pp. 252-270.

[43] Mark Pagel. "Inferring the historical patterns of biological evolution". In: Nature 401.6756 (1999), p. 877. 



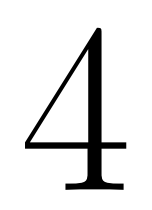

\section{A comparison of methods for gene-based testing that account for linkage disequilibrium}

Ozan Cinar and Wolfgang Viechtbauer. "A comparison of methods for gene-based testing that account for linkage disequilibrium". In: ((Submitted to) Statistics in Medicine). 


\section{Abstract}

Controlling the Type I error rate while retaining sufficient power is a major concern in genome-wide association studies, which nowadays often examine more than a million single-nucleotide polymorphisms (SNPs) simultaneously. Methods such as the Bonferroni correction can lead to a considerable decrease in power due to the large number of tests. Shifting the focus to higher functional structures (e.g., genes) can reduce the loss of power. This requires the combination of $p$-values of SNPs that belong to the same structural unit to test their joint null hypothesis. However, standard methods for this purpose (e.g., Fisher's method) do not account for the dependence among the tests due to linkage disequilibrium (LD). In this paper, we describe various adjustments to these methods that take LD into consideration and evaluated their performance in a simulation study based on data from the HapMap project. The results illustrate the importance of incorporating LD information into the methods. Furthermore, some methods are more successful in controlling the Type I error rate than others. Among them, Brown's method was the most robust technique with respect to the characteristics of the genes and outperformed the Bonferroni method in terms of power in many scenarios. 


\subsection{Introduction}

Genome-wide association (GWA) studies are commonly used to investigate the contribution of genetic variants to the risk of developing certain diseases [1]. In a typical GWA study, large quantities of single-nucleotide polymorphisms (SNPs) are genotyped to examine their association with some phenotype of interest (e.g., the presence or absence of a disease) or their interaction with some environmental factor $[2,3]$. However, the availability of genotype information for such a large number of SNPs will either lead to a high number of Type I errors or requires stringent corrections for multiple testing, which in turn inflates the number of Type II errors [4].

In particular, the probability of falsely rejecting an individual null hypothesis (e.g., that a SNP is unrelated to the outcome) is set a priori to a specific value by the researcher. This pointwise error rate (or error rate per hypothesis) is conventionally set to $\alpha_{p}=0.05$. However, the familywise error rate, $\alpha_{s}=$ $1-\left(1-\alpha_{p}\right)^{k}$ (i.e., the probability of falsely rejecting at least one of $k$ true null hypotheses) quickly increases when testing a large number of independent hypotheses. A common method to control the familywise error rate is the Bonferroni correction [5] that sets the pointwise error rate to $\alpha_{p} / k$, which in turn keeps $\alpha_{s}$ below the desired Type I error rate [6]. Considering that nowadays around a million SNPs are genotyped in a typical GWA study [1], the commonly used significance threshold of $5 \times 10^{-8}$ in such studies is loosely based on the Bonferroni correction [4, 7].

As a consequence of the decreased significance threshold, rejection of a null hypothesis becomes more difficult, whether it be a true null hypothesis or not. Therefore, the Bonferroni correction also increases the Type II error rate (i.e., the probability of failing to reject a false null hypothesis), which in turn decreases power. Although other multiple testing correction methods have been developed that lead to less severe reductions in power $[8,9,10,11$, $12,13]$, the reduction can still be severe due to the large number of SNPs considered in a typical GWA study [14].

A promising approach for mitigating this severe loss of power is to shift the focus of the analyses to higher functional structures such as genes (known as gene-based testing) or sets of genes that belong to common pathways [15]. As a result, the number of hypotheses tested declines dramatically (e.g., to 25,000-30,000 when testing at the gene level) and hence power is not as severely impacted when a correction for multiple testing is then applied. Furthermore, by aggregating signals from multiple SNPs, gene-based testing can 
be more appropriate for understanding the genetic structure of complex diseases $[16,17]$.

To test the significance of a gene as a whole, we need to test the joint null hypothesis of the SNPs that belong to the gene by combining their individual $p$-values into an overall $p$-value. A wide variety of methods have been described in the literature for combining independent tests of hypotheses [18, 19, 20, 21, 22, 23]. Among these, Fisher's method [24] may be the best-known one, which also has high relative efficiency asymptotically when compared to other methods [25, 26]. However, Fisher's method, like many other methods for combining tests of hypotheses, assumes that the $p$-values are independent of each other. This assumption is known to be violated in the present context as SNPs are often in linkage disequilibrium (LD), that is, the alleles at different loci exhibit non-random associations [27]. As a consequence, Fisher's method does not provide nominal results, typically leading to an inflation in the Type I error rate [28].

Several attempts have been made to adjust methods for combining $p$-values such that they take dependence into consideration. Brown [29] proposed an adjustment to Fisher's method for combining the results of dependent tests that has been used for gene-based testing [28, 30], whereas several other authors have described the use of principal component analysis (PCA) on the LD correlation matrix to estimate the effective number of tests $[31,32,33$, $34,35]$, which in turn can be combined with various multiple testing correction procedures. In addition, several authors have applied permutation tests or other permutation-type procedures to account for the dependence $[16,36]$. However, while permutation tests are often considered a 'gold standard' approach, such methods are computationally very demanding especially in GWA studies. Furthermore, proper permutation tests require access to the raw data which can be another limitation. A promising way to mimic the results of permutation tests (without needing the raw data and requiring a fraction of the time) is to generate pseudo replicates of the test statistics assuming they follow a multivariate normal distribution under the null hypothesis. These pseudo test statistics are then converted into SNP-level $p$-values which can be used to generate an empirical distribution of the combined $p$-value under the null hypothesis that takes the degree of LD into consideration [16, 37].

The statistical properties (i.e., Type I error rate and power) of these methods have been examined in previous research [4, 13, 28, 36, 38, 39, 40]. However, there are still several points that have not been considered so far. First, none of the studies have performed an extensive comparison among all methods on a 
genome-wide scale simultaneously. Furthermore, PCA-based approaches have only been combined with the Bonferroni correction (or with Tippett's method; see below), although they can also be used to modify other tests (e.g., Fisher's method) to account for dependence among the $p$-values. In addition, it is unknown whether the statistical properties of the correlations (e.g., their central tendency or spread) used to quantify the degree of LD might affect the performance of the methods. Moreover, some theoretical properties of the methods have not received sufficient attention. Most importantly, Brown's generalization of Fisher's method only applies to one-sided tests [29]. This property is especially problematic in GWA studies, since tests of the association between the SNPs and the phenotype of interest are typically two-sided [41]. An extension of Brown's method to two-sided tests has been described; however, its performance in the present context has yet to be investigated [42].

In this article, we review a variety of methods for combining $p$-values that can be used for gene-based testing and describe how LD can be incorporated into these methods. While doing so, an important goal is to provide a more complete description of how methods for combining $p$-values and adjustment techniques can be combined. For example, we will describe how an estimate of the effective number of tests can be used to adjust Fisher's method. Furthermore, we describe how all methods for combining $p$-values can be adjusted with an empirical distribution obtained using a pseudo-permutation approach. We also discuss the generalization of Brown's method to two-sided tests. Finally, we compare the Type I error rate and power of the methods based on a genome-wide Monte Carlo simulation study using LD matrices derived from the International HapMap Project [43].

\subsection{Materials and Methods}

For a collection of $i=1, \ldots, k$ SNPs that belong to a gene (or pathway), let $p_{1}, \ldots, p_{k}$ denote the $p$-values obtained when testing the association of each SNP with some phenotype of interest (or the interaction of each SNP with some other variable). We use $H_{0 i}$ to denote the null hypothesis corresponding to the $i$ th SNP. Since we are only interested in testing for association regardless of directionality, we assume that the $p$-values are derived from two-sided tests. Moreover, we assume that the tests have nominal properties, so that $p_{i} \sim$ Uniform $(0,1)$ when $H_{0 i}$ is true.

Instead of considering each of the $p$-values and null hypotheses individually, the goal is to combine the information from the individual tests into one that tests 
the gene as a whole. To be precise, the goal is to test the joint null hypothesis that none of the SNPs in the gene are associated with the phenotype (i.e., $H_{0 i}$ is true for all tests) against the alternative that at least one SNP is associated. We will now describe a variety of methods for this purpose.

\subsubsection{The Bonferroni Method}

The Bonferroni correction [5] is a method that was originally developed to control the familywise error rate when conducting multiple hypothesis tests. In order to apply the correction, the threshold for significance is adjusted by dividing the pointwise error rate, $\alpha_{p}$, by the number of simultaneous tests, $k$. Alternatively, we can adjust the individual $p$-values by multiplying them with $k$. Any test whose adjusted $p$-value is then equal to or less than $\alpha_{p}$ is declared significant [8].

Although not typically described in this manner, the Bonferroni method can also be used as a method for combining $p$-values. In particular, if any one of the adjusted $p$-values is significant, then the joint null hypothesis is automatically rejected. In the context of GWA studies, this means that if at least one SNP is significantly associated with the phenotype of interest, then the gene that this SNP belongs to is considered significant. Accordingly, the combined $p$-value for a gene is given by

$$
p=\min \left(1, \min \left(p_{1}, \ldots, p_{k}\right) \times k\right),
$$

where $\min (1, \ldots)$ simply ensures that the combined $p$-value cannot exceed 1 .

Contrary to popular belief, the Bonferroni correction does not make any assumptions about the degree of dependence among the $p$-values [44]. In other words, regardless of the degree of dependence among the tests from which the $p$-values are derived, the method guarantees that the Type I error rate is no larger than the desired nominal rate. This makes the methods particularly interesting for gene-based testing, where we know that the tests are likely to be dependent due to LD.

\subsubsection{Methods Assuming Independence}

In this subsection, we will describe methods that assume that the tests, and hence the $p$-values to be combined, are independent. Adjustments thereof will be considered later. 


\section{Tippett's Method}

Tippett's method [45], also known as the Dunn-Šidák correction for multiple testing [46, 47], follows from the fact that the familywise Type I error rate for $k$ independent tests, $\alpha_{s}=1-\left(1-\alpha_{p}\right)^{k}$, will equal a desired nominal rate, $\alpha$, if we set $\alpha_{p}=1-(1-\alpha)^{1 / k}$. Hence, the joint null distribution can be rejected if $\min \left(p_{i}\right) \leq 1-(1-\alpha)^{1 / k}$. Analogously, we can use

$$
p=1-\left(1-\min \left(p_{1}, \ldots, p_{k}\right)\right)^{k}
$$

as the $p$-value for the gene. As opposed to the Bonferroni method, which is slightly conservative even when all tests are independent, the method provides exact control of the Type I error rate, but only under independence.

\section{Binomial Test}

Under the joint null hypothesis, $r \sim \operatorname{Binomial}\left(k, \alpha_{p}\right)$ where $r$ denotes the number of tests that are significant at $\alpha_{p}$. Therefore, we can reject the joint null hypothesis if

$$
p=\sum_{x=r}^{k}\left(\begin{array}{l}
k \\
x
\end{array}\right) \alpha_{p}^{x}\left(1-\alpha_{p}\right)^{k-x}
$$

is equal to or less than the desired Type I error rate [21]. Intuitively, we can interpret this method as a test of "excess significance" of the SNPs within a gene. For example, the chances of finding $r=10$ or more significant SNPs at $\alpha_{p}=0.05$ in a gene with 100 independent SNPs is approximately $p=0.028$, which would be significant at $\alpha=0.05$.

\section{Fisher's Method}

Assuming that $p_{i} \sim \operatorname{Uniform}(0,1)$ under the null hypothesis of no association, it is easy to show that $-2 \ln \left(p_{i}\right)$ is chi-square distributed with 2 degrees of freedom. Hence, the combined test statistic

$$
X^{2}=-2 \sum_{i=1}^{k} \ln \left(p_{i}\right)
$$


follows a chi-squared distribution with $2 k$ degrees of freedom under the joint null hypothesis [24]. The $p$-value for the gene can therefore be computed with $p=1-F\left(X^{2}, 2 k\right)$, where $F(\cdot, 2 k)$ denotes the cumulative distribution function of a chi-square distribution with $2 k$ degrees of freedom.

\section{Stouffer's Method}

Let $\Phi(\cdot)$ denote the cumulative distribution function of the standard normal distribution and $\Phi^{-1}(\cdot)$ its inverse. Since $\Phi^{-1}\left(1-p_{i}\right)$ follows a standard normal distribution under $H_{0 i}, z=\sum_{i=1}^{k} \Phi^{-1}\left(1-p_{i}\right) / \sqrt{k} \sim \operatorname{Normal}(0,1)$ under the joint null hypothesis [20]. The $p$-value for a gene is then computed with $p=$ $1-\Phi(z)$.

\subsubsection{Incorporating Linkage Disequilibrium}

Except for the Bonferroni method, all methods described in the previous section assume that the tests are independent. Therefore, under this assumption (and the assumptions stated at the beginning of this section), these methods are guaranteed to have a Type I error rate equal to the desired $\alpha$ level (for the binomial test, the Type I error rate is $\leq \alpha$ due to the discrete nature of the binomial distribution). On the other hand, when the independence assumption is violated, the true Type I error rate may deviate from $\alpha$ in either direction, but usually leading to inflation (i.e., the joint null is rejected too often). In comparison, the Bonferroni method makes no assumptions about the degree of dependence among the tests and is guaranteed to have a rejection rate that is no larger than $\alpha$, but can be quite conservative under dependence. We will therefore now consider adjustments to the methods that account for dependence among the tests and that can bring their Type I error rate closer to $\alpha$.

\section{Effective Number of Tests}

One potential approach to adjust the previous methods is to quantify the degree of dependence between the tests, estimate the effective number of independent tests based on this information, and incorporate this estimate into the methods described above. 
The degree of dependence between the tests is closely related to the strength of the association between the SNPs. The latter can be quantified with various statistics (e.g., $D, D^{\prime}, r$, or $r^{2}$ ) expressing the degree of LD between pairs of SNPs [41]. We can use one of these measures to construct a $k \times k$ association matrix for all SNPs, sometimes called an "LD map". The effective number of tests can then be estimated based on this association matrix. A variety of approaches have been described in the literature for this purpose $[31,32,33$, $34,35]$. A common feature of all methods is that they start by applying PCA to the association matrix. We use $\lambda_{i}$ to denote the $i$ th eigenvalue extracted from the PCA.

The method proposed by Cheverud [31] and Nyholt [32] estimates the effective number of tests with

$$
k_{\mathrm{eff}}^{\mathrm{CN}}=1+(k-1)\left(1-\frac{\operatorname{Var}(\lambda)}{k}\right),
$$

where $\operatorname{Var}(\lambda)$ is the variance of the $k$ eigenvalues. On the other hand, Li and Ji [33] suggested the formula

$$
k_{\mathrm{eff}}^{\mathrm{LJ}}=\sum_{i=1}^{k} f\left(\left|\lambda_{i}\right|\right),
$$

where

$$
f(x)=I(x \geq 1)+(x-\lfloor x\rfloor)
$$

and $\lfloor\cdot\rfloor$ is the floor function. According to the method by Gao [34], we first sort the eigenvalues in decreasing order, letting $\lambda_{(1)}$ denote the largest and $\lambda_{(k)}$ the smallest eigenvalue. Then the effective number of tests is defined as

$$
k_{\mathrm{eff}}^{\mathrm{GAO}}=\min (x) \text { such that } \frac{\sum_{i=1}^{x} \lambda_{(i)}}{\sum_{i=1}^{k} \lambda_{(i)}}>C,
$$

where $C$ is a user-defined parameter and usually chosen to be 0.995 . Finally, Galwey [35] proposed to estimate the effective number of tests with

$$
k_{\mathrm{eff}}^{\mathrm{GAL}}=\frac{\left(\sum_{i=1}^{k} \sqrt{\lambda_{i}^{\prime}}\right)^{2}}{\sum_{i=1}^{k} \lambda_{i}^{\prime}},
$$

where $\lambda_{i}^{\prime}=\max \left(0, \lambda_{i}\right)$. 
All of the methods described above have the following desirable properties. When applied to an identity matrix (i.e., when there is no association between any pair of SNPs), then $k_{\text {eff }}=k$, so that the effective number of tests is equal to the number of SNPs. An exception to this property can occur with $k_{\text {eff }}^{\mathrm{GAO}}$. Depending on the value of $C$ and the number of tests, it can happen that the effective number of tests is then estimated to be less than $k$ (i.e., when $k(1-C)>1$ then $\left.k_{\text {eff }}^{G A O}<k\right)$. On the other hand, when all of the SNPs are perfectly associated (i.e., the correlation matrix is equal to a $k \times k$ matrix of 1 's), then $k_{\text {eff }}=1$. In essence, the same test is then repeated $k$ times, yielding identical results, so that effectively only a single test has been carried out. However, the methods differ in how association matrices that fall in between these two extremes are handled, yielding varying estimates of the effective number of tests between 1 and $k$.

Once $k_{\text {eff }}$ has been estimated with one of these approaches, it can be used to adjust each of the methods for combining $p$-values described earlier. For the Bonferroni and Tippett's methods, we substitute $k_{\text {eff }}$ for $k$ so that

$$
p=\min \left(1, \min \left(p_{1}, \ldots, p_{k}\right) \times k_{\mathrm{eff}}\right) .
$$

and

$$
p=1-\left(1-\min \left(p_{1}, \ldots, p_{k}\right)\right)^{k_{\text {eff }}}
$$

are then the $p$-values for the gene. For the binomial test, we first define $\tilde{r}=$ $\left\lfloor r \times \frac{k_{\text {eff }}}{k}\right\rfloor$ as the adjusted (i.e., effective) number of significant SNPs within the gene. Then the $p$-value for the gene is computed with

$$
p=\sum_{x=\tilde{r}}^{k_{\mathrm{eff}}}\left(\begin{array}{c}
k_{\mathrm{eff}} \\
x
\end{array}\right) \alpha_{p}^{x}\left(1-\alpha_{p}\right)^{k_{\mathrm{eff}}-x} .
$$

Use of the floor function for computing $\tilde{r}$ may be conservative, but we consider this preferable over rounding and the risk of a too liberal test. Fisher's method can be adjusted by replacing the degrees of freedom of the chi-square distribution with $2 k_{\text {eff }}$ and adjusting the test statistic with $\tilde{X}^{2}=\frac{k_{\text {eff }}}{\underline{k}} \times X^{2}$. Hence, the $p$-value for the gene is then computed with $p=1-F\left(\tilde{X}^{2}, 2 k_{\text {eff }}\right)$. Finally, for Stouffer's method, we let $\tilde{z}=\sqrt{\frac{k_{\text {eff }}}{k}} \times z$ denote the adjusted test statistic and hence $p=1-\Phi(\tilde{z})$ is then the $p$-value for the gene. 


\section{Methods Based on Empirically-Derived Null Distributions}

Another approach to account for dependence is to make use of permutation testing $[4,28]$. The idea is to empirically derive the null distribution of the test statistic of interest by reshuffling the data in such a way that relevant features of the data structure are preserved except for the actual association being tested. For example, when testing for the association between each SNP and case-control status, reshuffling the status variable breaks any existing associations, but keeps the LD structure of the SNPs intact. Hence, any dependence among the $p$-values to be combined using one of the methods described earlier is automatically incorporated into the null distribution. The $p$-value for a gene is then computed from the percentile of the actually observed test statistic under the empirical null distribution. Note that in the present case, the test statistic of interest is actually a $p$-value itself, so letting $p_{j}$ denote the combined $p$-value based on the $j$ th permutation of the data (with $j=1, \ldots, s$ ) and $p_{\text {obs }}$ the observed combined $p$-value, the $p$-value for a gene is then given by $p=\sum_{j=1}^{s} I\left(p_{j} \leq p_{\text {obs }}\right) / s$.

Permuting the data in the manner described above requires access to the raw data, so that the phenotype variable can be reshuffled and the test of association can be conducted for each SNP. In addition, repeatedly computing the test of association for each SNP within a gene can be computationally demanding. We can reduce the computational burden and eliminate the dependence upon the raw data by directly generating $p$-values based on an association matrix that reflects the degree of LD among the SNPs [16] which may be obtained from a reference population and not necessarily the given data.

In particular, let $R$ denote the $\mathrm{LD}$ association matrix constructed from the correlations among the SNPs. We can then quickly generate a large number $(s)$ of samples from a multivariate normal distribution with a true mean vector equal to zeros and covariance matrix $R$. Let $Z$ denote the $s \times k$ matrix of these values and $P=2(1-\Phi(|Z|))$ the matrix of two-sided $p$-values obtained by applying $\Phi(\cdot)$ element-wise. For each row in $P$, we then apply one of the methods for combining $p$-values, yielding $p_{j}$. The $p$-value for a gene is then again computed as described above.

\section{Methods Derived under Dependence}

The last set of methods we will consider are modifications of Fisher's and Stouffer's method so that dependence among the tests is directly taken into 
consideration.

Brown's Method. The first adjustment is based on Brown [29] who proposed a modification of Fisher's method for combining the results of correlated one-sided $z$-tests. If the $p$-values are not independent, $X^{2}$ has expected value $\mathrm{E}\left(X^{2}\right)=2 k$ and variance $\operatorname{Var}\left(X^{2}\right)=4 k+2 \sum_{i=1}^{k-1} \sum_{j>i}^{k} \operatorname{Cov}\left(-2 \ln \left(p_{i}\right),-2 \ln \left(p_{j}\right)\right)$, where the covariance between two $-2 \ln (\cdot)$-transformed $p$-values is given by

$$
\begin{aligned}
& \operatorname{Cov}\left(-2 \ln \left(p_{i}\right),-2 \ln \left(p_{j}\right)\right) \\
& =4 \int_{-\infty}^{+\infty} \ln \left(1-\Phi\left(z_{i}\right)\right) \times \ln \left(1-\Phi\left(z_{j}\right)\right) f\left(z_{i}, z_{j}\right) \mathrm{d} z_{i} \mathrm{~d} z_{j}-4,
\end{aligned}
$$

where $\left(z_{i}, z_{j}\right)$ is assumed to follow a bivariate standard normal distribution with correlation equal to the correlation among the two SNPs and $f\left(z_{i}, z_{j}\right)$ denotes the joint probability density function of this distribution. The covariance term can be computed using numerical integration, although Brown [29] also proposed a closed-form approximation that avoids this step. Next, we assume that $X^{2}$ follows a scaled chi-squared distribution, i.e., $X^{2} \sim c \chi_{f}^{2}$ (or equivalently, $X^{2} / c \sim \chi_{f}^{2}$ ), where $\chi_{f}^{2}$ denotes a chi-squared distributed random variable with $f$ degrees of freedom, and then approximate this distribution by equating its first two moments to the expected value and variance of $X^{2}$ as calculated above. That is, for $X^{2} \sim c \chi_{f}^{2}$, it follows that $\mathrm{E}\left(X^{2}\right)=c f$ and $\operatorname{Var}\left(X^{2}\right)=2 c^{2} f$, which implies $f=2\left(\mathrm{E}\left(X^{2}\right)\right)^{2} / \operatorname{Var}\left(X^{2}\right)$ and $c=\operatorname{Var}\left(X^{2}\right) / 2 \mathrm{E}\left(X^{2}\right)$. The $p$-value for a gene is then computed with $p=1-F\left(X^{2} / c, f\right)$, where $F(\cdot, f)$ denotes the cumulative distribution function of a chi-square distribution with $f$ degrees of freedom.

As given above, the method is only applicable to one-sided tests. However, in GWA studies, the association between the phenotype and the SNPs is typically examined with two-sided tests. We can easily extend Brown's method to twosided tests by computing the covariance with

$$
\begin{aligned}
& \operatorname{Cov}\left(-2 \ln \left(p_{i}\right),-2 \ln \left(p_{j}\right)\right) \\
& =4 \int_{-\infty}^{+\infty} \ln \left(2\left(1-\Phi\left(\left|z_{i}\right|\right)\right)\right) \times \ln \left(2\left(1-\Phi\left(1-\left|z_{j}\right|\right)\right)\right) f\left(z_{i}, z_{j}\right) \mathrm{d} z_{i} \mathrm{~d} z_{j}-4,
\end{aligned}
$$

with $\left(z_{i}, z_{j}\right)$ and $f\left(z_{i}, z_{j}\right)$ as defined above [42]. The remaining steps of the 
method are unchanged.

Strube's Method. Finally, Stouffer's method can also be generalized to consider the dependence among tests [48]. To do so, we assume (as in Brown's method) that the test statistics that generated the $p$-values follow a multivariate normal distribution where the correlations among the test statistics are given by the correlations among the SNPs. We then compute

$$
z=\frac{\sum_{i=1}^{k} \Phi^{-1}\left(1-p_{i}\right)}{\sqrt{\operatorname{Var}\left(\sum_{i=1}^{k} \Phi^{-1}\left(1-p_{i}\right)\right)}},
$$

where $\operatorname{Var}\left(\sum_{i=1}^{k} \Phi^{-1}\left(1-p_{i}\right)\right)=k+2 \sum_{i=1}^{k-1} \sum_{j>i}^{k} \operatorname{Cov}\left(\Phi^{-1}\left(1-p_{i}\right), \Phi^{-1}(1-\right.$ $\left.\left.p_{j}\right)\right)$ ). The challenge is again the computation of the covariance term, which in this case is given by

$$
\begin{aligned}
& \operatorname{Cov}\left(\Phi^{-1}\left(1-p_{i}\right), \Phi^{-1}\left(1-p_{j}\right)\right) \\
& =\int_{-\infty}^{+\infty} \int^{-1}\left(1-2\left(1-\Phi\left(\left|z_{i}\right|\right)\right)\right) \times \Phi^{-1}\left(1-2\left(1-\Phi\left(\left|z_{j}\right|\right)\right)\right) f\left(z_{i}, z_{j}\right) \mathrm{d} z_{i} \mathrm{~d} z_{j}
\end{aligned}
$$

and which can again be computed using numerical integration. Then the combined $p$-value is calculated with $p=1-\Phi(z)$.

\subsubsection{Illustrative Example}

The methods for combining $p$-values described in the previous section can yield conflicting conclusions. In particular, while the (unadjusted) Bonferroni method controls the Type I error rate even under dependence, it may fail to detect significant associations when combining non-independent $p$-values due to its conservative behaviour in such contexts. In this section, we present an example to illustrate this point.

Van Assche and colleagues [49] reported the results of a candidate gene study based on a sample of 982 Caucasian adolescents, analyzing 4947 SNPs clustered in 263 genes known to be involved in neurotransmission. The outcome of interest was the (log-transformed) score on the Center for Epidemiologic Studies Depression Scale [50]. The association between each SNP and the 
Chapter 4. A comparison of methods for gene-based testing that account for linkage disequilibrium

Table 4.1: Combined $p$-values for the GRID2IP and ARNTL2 genes based on the methods presented in section 2 (combined $p$-values that show non-significant associations are accentuated in italic).

\begin{tabular}{|c|c|c|c|c|c|c|c|}
\hline GRID2IP & Unadjusted & $\begin{array}{c}\text { Cheverud- } \\
\text { Nyholt }\end{array}$ & $\mathrm{Li}$ and $\mathrm{Ji}$ & Gao & Galwey & $\begin{array}{c}\text { Empirically } \\
\text { Derived }\end{array}$ & $\begin{array}{c}\text { Under } \\
\text { Dependence }\end{array}$ \\
\hline$k=23$ & & $k_{\mathrm{eff}}^{\mathrm{CN}}=20$ & $k_{\text {eff }}^{L J}=15$ & $k_{\mathrm{eff}}^{\mathrm{GAO}}=18$ & $k_{\mathrm{eff}}^{\mathrm{GAL}}=13$ & & \\
\hline Bonferroni & 0.068 & 0.060 & 0.045 & 0.054 & 0.039 & 0.052 & \\
\hline Tippett & 0.066 & 0.058 & 0.044 & 0.052 & 0.038 & 0.051 & \\
\hline Binomial & $<0.001$ & $<0.001$ & $<0.001$ & $<0.001$ & $<0.001$ & $<0.001$ & \\
\hline Fisher & $<0.001$ & $<0.001$ & $<0.001$ & $<0.001$ & $<0.001$ & 0.002 & 0.001 \\
\hline Stouffer & $<0.001$ & $<0.001$ & $<0.001$ & $<0.001$ & $<0.001$ & 0.002 & $<0.001$ \\
\hline ARNTL2 & Unadjusted & $\begin{array}{c}\text { Cheverud- } \\
\text { Nyholt }\end{array}$ & $\mathrm{Li}$ and $\mathrm{Ji}$ & Gao & Galwey & $\begin{array}{c}\text { Empirically } \\
\text { Derived }\end{array}$ & $\begin{array}{c}\text { Under } \\
\text { Dependence }\end{array}$ \\
\hline$k=24$ & & $k_{\mathrm{eff}}^{\mathrm{CN}}=22$ & $k_{\text {eff }}^{L J}=14$ & $k_{\mathrm{eff}}^{\mathrm{GAO}}=18$ & $k_{\mathrm{eff}}^{\mathrm{GAL}}=14$ & & \\
\hline Bonferroni & 0.112 & 0.103 & 0.065 & 0.084 & 0.065 & 0.082 & \\
\hline Tippett & 0.106 & 0.098 & 0.063 & 0.081 & 0.063 & 0.082 & \\
\hline Binomial & $<0.001$ & 0.001 & 0.004 & 0.002 & 0.004 & 0.011 & \\
\hline Fisher & $<0.001$ & $<0.001$ & 0.003 & 0.001 & 0.003 & 0.020 & 0.016 \\
\hline Stouffer & 0.001 & 0.001 & 0.009 & 0.003 & 0.009 & 0.030 & 0.023 \\
\hline
\end{tabular}

outcome was tested using an additive model [41]. The resulting $p$-values were then combined within each gene using Brown's method. The results showed that a small number of genes were significantly associated with the phenotype at $\alpha=0.05$.

For illustration purposes, we obtained the combined $p$-values for two genes (GRID2IP and ARNTL2) with all of the methods described above. LD maps were calculated with the genetics package in $\mathrm{R}$ [51], using the allelic correlation to measure the degree of association between the SNPs within each gene. The combined $p$-values were then obtained using the poolr package in $\mathrm{R}$ [52]. Empirical null distributions were generated as described earlier using $s=10^{6}$ samples.

Table 4.1 presents the combined $p$-values for the two genes. Heat maps corresponding to the LD structure for these two genes (and the individual $p$-values for the SNPs) are provided in Figure S1 as part of the supplementary materials. The table shows that the Bonferroni method fails to detect a significant association between both genes and the phenotype, whereas other approaches (including Brown's method) suggest a significant association. Interestingly, adjusting the Bonferroni method with two of the PCA-based methods (i.e., $k_{\text {eff }}^{\mathrm{LJ}}$ and $k_{\text {eff }}^{\mathrm{GAL}}$ ) leads to a significant finding at least for GRID2IP.

While this example demonstrates that conclusions can differ depending on the method used, we do not know which of the conclusions drawn above are correct. 
In other words, the (non-significant) results of the Bonferroni method may be Type II errors (which are then avoided by using other methods) or they may be true negatives (with other methods then leading to Type I errors). Therefore, to further examine the performance of the various methods when we do know whether the null hypothesis is true or not, we conducted a simulation study.

\subsubsection{Simulation Study}

We based the simulation on HapMap phase II+III data [43] so that the results are representative of real genotype and LD information. Since genetic recombination breaks down disequilibria among the SNPs over time, LD tends to be weaker in older populations [53]. We therefore used information from the TSI (Italian) sample from a somewhat younger population to avoid LD maps overwhelmed with negligible pairwise LD values. The sample contained $n=102$ individuals and 1,421,526 SNPs with their chromosome and position information.

We focus on autosomal chromosomes and excluded the sex chromosomes. Furthermore, insertions and deletions (INDELs) in the data [54] were removed. SNPs were assigned to genes using the biomaRt package in $\mathrm{R}$ through the Ensembl database $[55,56,57]$. SNPs that were not assigned to a gene were excluded while SNPs that were assigned to multiple genes (due to overlapping genes) were kept in the study. After the assignment of SNPs to genes, the data included 915,259 SNPs in 30,910 genes. The number of SNPs per gene ranged from 2 to 3178 with a mean (SD) of 29.61 (68.68) and a median of 12 . Missing genotypes were then imputed using the MaCH software [58]. Next, LD maps (using allelic correlations) were computed for these genes using the LD() function of the genetics package in $\mathrm{R}$ [51]. For LD maps that were not positive definite, the nearest positive definite correlation matrices were obtained with the nearPD() function of the Matrix package [59]. Finally, genotypes were coded in an additive manner (i.e., 0/1/2 coding), corresponding to the number of minor alleles at a locus [28].

For each gene, we then examined the Type I error rate of the various methods by (a) simulating a dichotomous phenotype variable (e.g., case-control status) for the $n=102$ individuals based on a Bernoulli distribution with $\pi=0.50$, (b) testing the association between each SNP within the gene and the phenotype variable using the Cochran-Armitage trend test [60], (c) combining the resulting $p$-values with each method described earlier, (d) repeating this process 1000 times, and (e) calculating the proportion of times that the gene is 
declared significant at $\alpha=0.05$ according to each method. For methods that make use of empirical distributions, we generated these distributions as described earlier using $s=100,000$ samples. Since the Cochran-Armitage trend test does not require that the Hardy-Weinberg equilibrium (HWE) assumption holds [41], we did not filter out SNPs that violate this assumption.

To examine the power of the methods, the same steps as described above were repeated, but the probability of having case status was now made a logistic function of one or multiple SNPs within the gene, that is, for the $j$ th individual in the data set, we set the probability of having case status equal to

$$
\pi_{j}=\frac{\exp \left(\beta_{0}+\sum_{i=1}^{k} x_{i j} \beta_{i}\right)}{1+\exp \left(\beta_{0}+\sum_{i=1}^{k} x_{i j} \beta_{i}\right)},
$$

where $x_{i j}$ denotes the number of minor alleles for the $i$ th SNP of the $j$ th individual, $\beta_{i}$ determines how strongly the $i$ th SNP is related to case-control status, and

$$
\beta_{0}=-\frac{\sum_{j=1}^{n} \sum_{i=1}^{k} x_{i j} \beta_{i}}{n},
$$

so that approximately half of the $n$ individuals were assigned to case status and the other half were controls.

This part of the simulation involved several scenarios with different features. In the first set of conditions, a single SNP within a given gene was chosen in each iteration and the corresponding $\beta_{i}$ value was set to either 0.2 or 0.5 (two different conditions). In the remaining conditions, we either allowed $5 \%$ or $20 \%$ of the SNPs within each gene to be associated with the phenotype. We again examined two different effect sizes $(0.2$ or 0.5$)$ and either set $\beta_{i}$ to the effect size value for each selected SNP (non-distributed effect) or distributed the effect over all selected SNPs (e.g., $\beta_{i}=0.2 / 10$ for an effect size of 0.2 and 10 selected SNPs). Finally, the selected SNPs were either positioned on a compact region of the gene (for this, a single SNP was randomly chosen among the first $k \times(1-.05)$ or $k \times(1-.20)$ SNPs and all consecutive SNPs were then also selected) or were dispersed throughout the gene (for this, significant SNPs were equally spaced throughout the gene). Hence, in addition to the first two conditions where a single SNP was associated with the phenotype with either an effect size of 0.2 or 0.5 , we examined another 16 conditions, as all factors $(5 \%$ vs $20 \%$ of SNPs selected, effect size of 0.2 vs 0.5 , non-distributed vs distributed effect, non-compact vs compact SNP selection) were fully crossed.

To examine how sample size impacts the Type I error rate and power of the 
methods, the same steps were repeated but in each iteration, a bootstrap sample of size 102, 500, or 1000 was generated from the original data (together with the non-bootstrap conditions, we therefore examined 4 different sample size conditions). We included a condition with a bootstrap sample size of 102 to examine whether the performance of the methods differed whether the original data or a bootstrap sample of the same size was used. In total, we therefore examined a total of $\left(1_{\text {Type I error }}+2_{\text {Single SNP }}+16_{\text {Multiple SNPs }}\right) \times 4_{\text {Sample Size }}=76$ different conditions.

The simulation was carried out using $\mathrm{R}$ [61] and was run on a cluster computer, making use of 144 cores (12 Intel Xeon E5-2650 2.20GHz CPUs with 12 cores each) using parallel/multicore processing. Total computation time for the simulation was approximately 20,000 core hours.

\subsection{Results}

Figure 4.1 shows boxplots of the Type I error rates of all methods observed on all 30,910 genes applied to the original HapMap dataset (i.e., based on the non-bootstrapped data). Individual genes are indicated as points when their rate was more than 1.5 times the interquartile range above the third or below the first quartile. The mean rejection rates (and SDs) are also indicated in the figure.

None of the unadjusted methods could achieve on average a nominal rejection rate of $\alpha=.05$. As expected, the Bonferroni method tended to be conservative, as was Tippett's method, which produced very similar results to the former method throughout the entire simulation study and which will therefore not be further discussed (note that rates above .05 for the Bonferroni method which occurred for about $1.5 \%$ of the genes - reflect simulation error, since we know that the method guarantees that the Type I error rate is equal to or less than $\alpha$ regardless of the degree of dependence). On the other hand, the remaining methods were generally liberal, at times dramatically so, with the binomial test at least providing an average rejection rate closest to the nominal level.

The adjustments for addressing the dependence did bring the rejection rates closer to the nominal level with varying degrees of success. In fact, when adjusted with the Li \& Ji method, the Bonferroni method had a nominal average rejection rate, although this came at the cost of increased variability in the Type I error rates and the occurrence of rates well above the nominal 


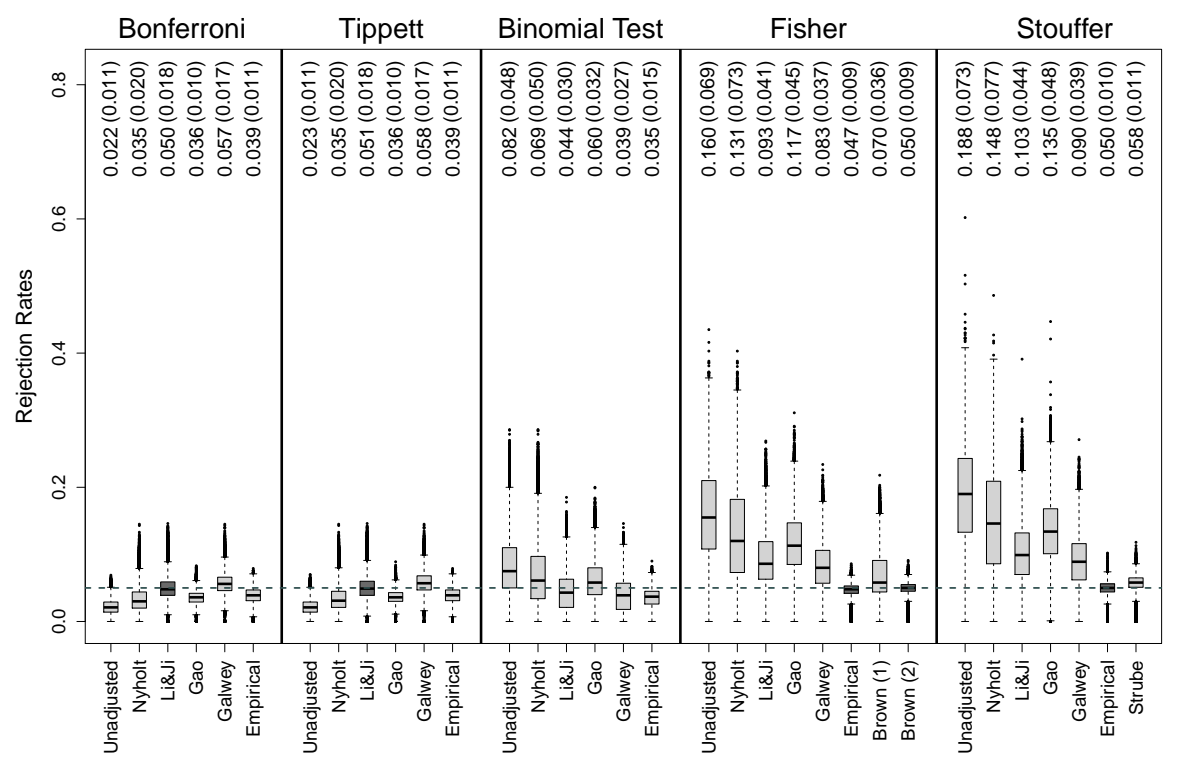

Figure 4.1: Type I error rates of methods for gene-based testing when applied to the original HapMap data. The numbers above the boxplots show the mean (SD) rejection rates of the methods. The horizontal grey dashed line corresponds to the nominal rejection rate of $\alpha=0.05$.

level for particular genes. For the binomial test, the average rates fluctuated around the nominal level, being slightly conservative with the Li \& Ji and Galwey adjustments and slightly liberal with the Nyholt and Gao adjustments. In contrast, none of the PCA-based adjustments could bring the average Type I error rates of the Fisher and Stouffer methods sufficiently close to $\alpha=.05$.

Using empirically-derived null distributions produced rejection rates that were on average reasonably close to the nominal level, especially for Fisher's and Stouffer's methods. Moreover, the Type I error rates of individual genes had much lower variability than the rates obtained with the PCA-based adjustments. This was also true for the Bonferroni method and the binomial test, but these methods were slightly conservative on average when adjusted in this manner.

The (two-sided) generalization of Fisher's method to dependent tests (i.e., 
Brown's method) yielded a nominal rejection rate on average. Furthermore, the variability (i.e., SD) of the rates for individual genes was lowest compared to all other methods. Quite importantly, (mis)application of the one-sided version of Brown's method (since the $p$-values for the SNPs were computed from two-sided tests) resulted in worse performance (further references to Brown's method will therefore pertain to the two-sided version unless otherwise stated). On the other hand, the generalization of Stouffer's method to dependent tests (i.e., Strube's method) performed reasonably well, although its Type I error rate was on average slightly inflated.

Figures S2-S4 show the Type I error rates of the methods based on bootstrap samples of sizes 102, 500, and 1000, respectively. A comparison of Figures 4.1 and S2 (both with $n=102$ ) shows that the performance of the methods was similar regardless of whether they were applied to the original data or to bootstrap samples of the same size. The only exception to this was Stouffer's method, which became slightly more conservative for the bootstrapped data. Also, the Type I error rates of the methods were not fundamentally altered when applied to larger sample sizes. Using empirical distributions in combination with Fisher's and Stouffer's methods and Brown's method generally resulted in adequate control of the Type I error rate on average and comparatively low variability in the rates for individual genes.

To examine whether the performance of the methods was affected by certain characteristics of the genes, we examined their Type I error rates as a function of the (log transformed) number of SNPs in the genes, the average correlation in the LD maps, the degree of variability (i.e., standard deviation) of the correlations, the square-root of the mean squared correlations (SRMSC), the average minor allele frequencies (MAF) of the SNPs in the genes, and the standard deviation (SD) of the MAFs. The SRMSC was of particular interest as it distinguishes genes whose SNPs are independent (SRMSC equal to 0) from genes with SNPs in strong LD regardless of the directionality of the association (SRMSC close to 1). We used locally estimated scatterplot smoothing to visualize the relationship between these characteristics and the rejection rates for each method.

Figure 4.2 shows that the Type I error rate of many methods was affected by the number of SNPs in the genes. In particular, the Bonferroni method became increasingly conservative as the number of SNPs increased. Interestingly, this dependence on $k$ was essentially removed when using the adjustment of Li \& $\mathrm{Ji}$ and, to a slightly lesser extent, the adjustment of Gao. In contrast, the binomial test and Fisher's method became increasingly liberal as a function of 

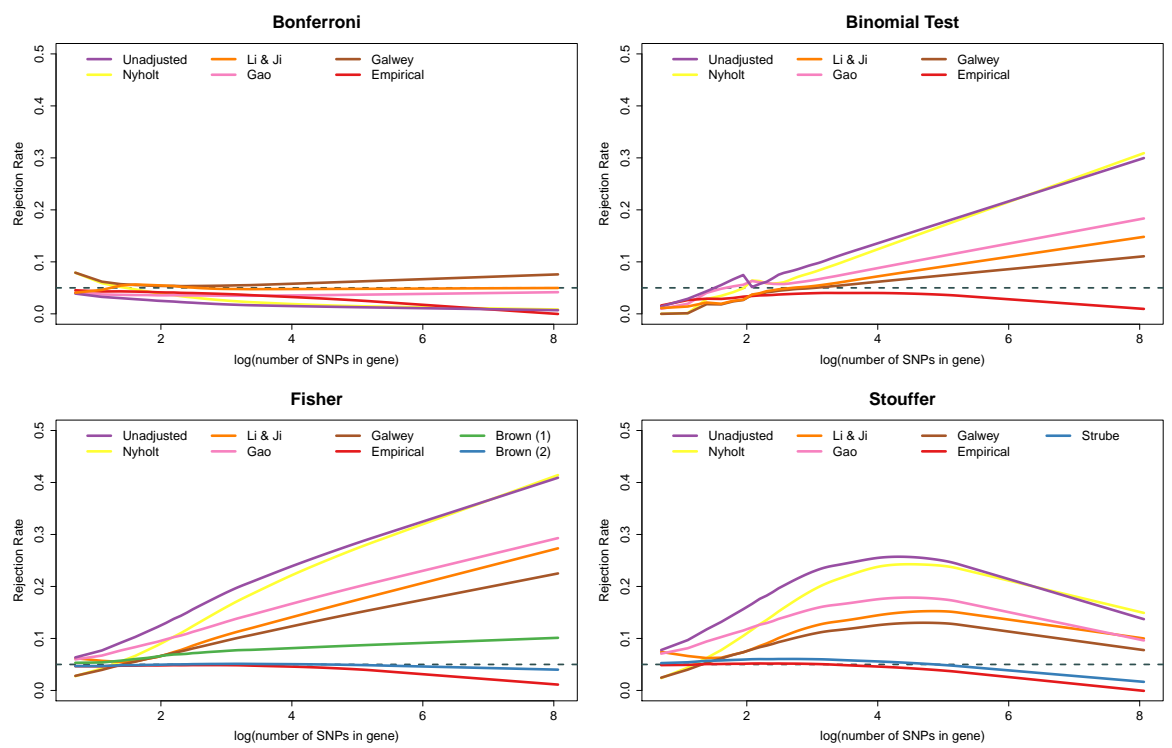

Figure 4.2: Type I error rates of methods for gene-based testing as a function of the number of SNPs in the genes (log-transformed). The horizontal grey dashed line corresponds to the nominal rejection rate of $\alpha=0.05$.

$k$, whereas Stouffer's method displayed non-monotonic behaviour. The PCAbased adjustments helped to reduce the inflation in the Type I error rates of these methods, but could not eliminate the dependence on $k$. Furthermore, all methods adjusted based on empirical distributions became increasingly conservative as the number of SNPs increased. Finally, Brown's method yielded essentially nominal rates regardless of $k$, except for very large genes, where the method became slightly conservative.

Figure 4.3 shows the Type I error rates as a function of the SRMSC values. As expected, the figure points out the increasingly conservative behavior of the Bonferroni method as the SNPs within the genes become more dependent, while Fisher's and Stouffer's methods then become liberal. The conservative behavior of the binomial test under independence is also expected (due to the discrete nature of the binomial distribution, the Type I error rate of the test will not exceed $\alpha=.05$, but will often fall well below it). More surprising is the fact that the Type I error rate of the method was essentially nominal for 

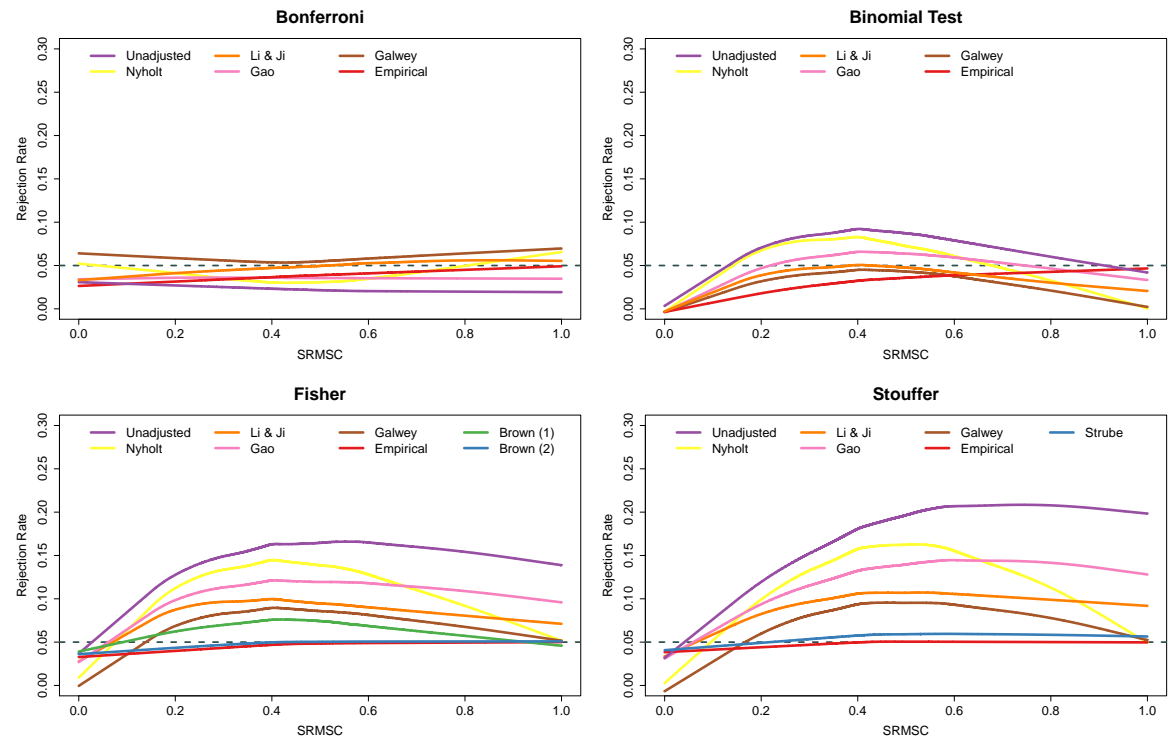

Figure 4.3: Type I error rates of methods for gene-based testing as a function of the square-root mean squared correlation (SRMSC). The horizontal grey dashed line corresponds to the nominal rejection rate of $\alpha=0.05$.

genes with very strong LD. To understand this phenomenon, consider a gene with $k$ SNPs in perfect LD. In that case, all (two-sided) $p$-values are identical and hence either none or all $k$ SNPs are significant. Since the latter will happen (under the joint null) with probability $\alpha$, the test will exhibit nominal performance under this extreme scenario. With respect to the adjustments, the various PCA-based approaches again had the effect of counteracting the conservativeness of the Bonferroni method, while leading to a reduction in the Type I error rates of the other methods. For all methods, adjusting with the use of empirical distributions was most successful when LD is strong, while Brown's method, although slightly conservative under independence, performed well over the range of SRMSC values. Strube's method performed similarly, but with some slight inflation for larger SRMSC values.

Figures S5 and S6 show the Type I error rates of the methods as a function of the average correlation and the SD of the correlations of the SNPs within the genes. Here, we again find that changes in these characteristics have essentially 
no impact on the performance of Brown's method, as well as when using empirical distributions in combination with Fisher's and Strube's methods. Interestingly, the one-sided version of Brown's method performed similarly to the two-sided one when the average $\mathrm{LD}$ was larger than $\approx 0.3$.

Figures S7 and S8 display the Type I error rates of the methods as a function of the average MAFs and their SD within the genes. The performance of Brown's method and Fisher's method with the empirical distribution adjustment was again not affected substantially by these factors except that these methods were slightly conservative when the average MAF was below 0.1 within the genes. Stouffer's method adjusted based on empirical distributions and its generalization to dependence was also relatively robust to both factors.

Figure 4.4 illustrates the power of the methods (averaged over genes) for the 54 conditions where the joint null hypothesis was false. Each panel corresponds to one of three sample size conditions (i.e., 102, 500, and 1000), while the x-axis indicates the condition, starting with the two 'single SNP' conditions (with effect sizes of 0.2 vs 0.5 ) followed by the 16 'multiple SNPs' conditions (with either $5 \%$ or $20 \%$ of SNPs selected, an effect sizes of 0.2 or 0.5 , a non-distributed or distributed effect, and either non-compact or compact SNP positions). We only show the power rates for the (unadjusted) Bonferroni method and those method and adjustment combinations that could control the Type I error rate on average (i.e., the Bonferroni method with the Li \& Ji adjustment, Brown's method, and use of empirical distributions in combination with Fisher's and Stouffer's methods).

As expected, power increased with the sample size, with the effect size, when a higher percentage of SNPs was associated with the phenotype, and when the effect was not distributed across these SNPs. Whether the selected SNPs fell into a compact region of the gene or were distributed throughout had comparatively little influence on the results. The (unadjusted) Bonferroni method typically had lower power compared to the other methods especially for lower sample sizes. Also, Stouffer's method adjusted with the use of empirical distributions tended to have slightly lower power compared to the other adjusted methods. While differences between the other methods were often negligible, a slight power advantage could be observed for the Bonferroni method adjusted with the $\mathrm{Li} \&$ Ji correction when a single SNP or a low percentage of them contained a strong signal. Otherwise, Brown's method tended to have a slight power advantage. 

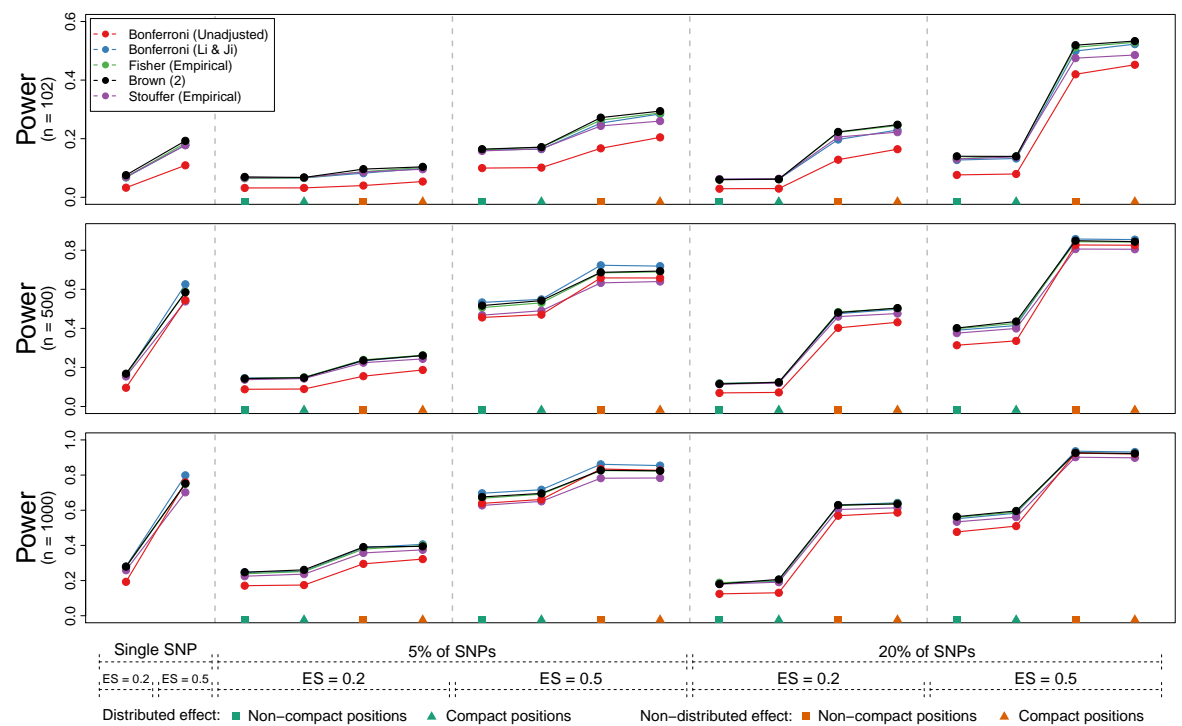

Figure 4.4: Power comparison of five methods for gene-based testing for three different sample sizes $(n=102,500$, and 1000). The points represent the average rejection rate of a method under a specific scenario as indicated by the labels/symbols under the $\mathrm{x}$-axis (number/percent of SNPs that are associated with the phenotype, the effect size, whether the effect was distributed over the significant SNPs, and whether significant SNPs were compactly positioned within the genes).

\subsection{Discussion}

In this paper, we described some common methods for gene-based testing that combine the $p$-values of individual SNPs within genes (or that are clustered within some other higher-level functional structure) to test the joint null hypothesis that none of the SNPs within a gene are associated with the phenotype of interest. Along the way, we described a variety of adjustment techniques to incorporate LD information into this process. To examine and compare the Type I error rates and power of the methods, we conducted an extensive simulation study based on HapMap data. While the (unadjusted) Bonferroni method guarantees that the Type I error rate is never larger than the chosen significance level for all genes, the results show that this comes at the cost of a decrease in power for detecting genes that contain SNPs associated with the 
phenotype of interest.

Other methods for gene-based testing require adjustments based on the LD structure to ensure that their Type I error rate is close to the nominal level on average. Doing so can increase the power for detecting 'significant genes', but this in turn can lead to an inflated Type I error rate for some of the individual genes. We would consider this an acceptable risk under two conditions. First, the variability in the rates for individual genes should be low (to avoid excessively inflated Type I error rates for particular genes). Moreover, the method should provide adequate control of the Type I error rate regardless of the characteristics of the genes.

Among the various methods examined, the extension of Brown's method to two-sided tests comes closest to fulfilling these requirements. It had a nominal Type I error rate on average and the lowest variability in the rates for individual genes (also when compared against the unadjusted Bonferroni method). The highest Type I error rate observed across all 30,910 genes was .091, but this value might reflect at least in part simulation error, as the Bonferroni method also had inflated rates for $470(1.5 \%)$ of the genes, with a maximum rate equal to .069. To further examine this, we repeated the simulation for these 470 genes using 1,000,000 iterations (see Figure S9 for boxplots of the Type I error rates of the Bonferroni and Brown's method). Now, only 34 of these genes still had a Type I error rate above .05 with the Bonferroni method, with a maximum rate of .056. In contrast, the highest Type I error rate of Brown's method was then .058, although (as expected) a higher number (188 out of these 470 genes) still had a rate above .05. Finally, the results based on all 30,910 genes showed that the Bonferroni method became increasingly conservative for genes with a larger number of SNPs or SNPs that were is stronger LD, while the performance of Brown's method was essentially independent of the various gene characteristics examined (except for some slight conservativeness when the degree of LD was very weak).

Another consideration in this context is the relative performance of the methods depending on whether the 'signal' is concentrated in a single SNP or distributed over a larger number of them. The Bonferroni method - which focuses on the lowest $p$-value among the SNPs within a gene - might be at an advantage under the former scenario, while methods that can aggregate signals across multiple SNPs (such as Fisher's and Stouffer's methods and versions thereof adjusted to account for dependence) would be expected to be more powerful in the latter case. However, under the conditions studied, the (unadjusted) Bonferroni method was never able to outperform Brown's method even when 
only a single SNP was strongly associated with the phenotype. Only when combined with the adjustment by $\mathrm{Li} \& \mathrm{Li}$ did the Bonferroni method show a slight power advantage under this scenario. Brown's method may therefore be particularly advantageous when studying complex diseases where relatively small associations are likely to be spread across many SNPs and multiple genes $[28,62]$.

We also considered how an estimate of the effective number of tests can be used to adjust other methods besides the Bonferroni or Tippett methods (to which such adjustments are typically applied). However, none of these generalizations yielded nominal Type I error rates on average. On the other hand, combining the Bonferroni method with the estimate of Li \& Ji [33] did perform adequately and, as mentioned above, may be of interest when the signal is concentrated in a single SNP. Our findings are in line with those by Wen and Lu [39] who showed that the method by Li \& Ji performs better than other effective number of tests adjustments.

Finally, we explored methods that mimic 'proper' permutation tests by using pseudo replicates of the $p$-values to construct the empirical distributions needed for such tests. This approach greatly reduces the computation time (and can even be used when the raw data are not available) and produces results that are quite similar to those of conventional permutation techniques. [13, 16, 28, 36]. However, the results of our simulation study show that the performance of this approach depends on the method used for combining the $p$-values. Moreover, the Type I error rate of these pseudo permutation tests either tended to be slightly conservative or, when the Type I error rate was nominal on average, they offered no power advantage over Brown's method.

In conclusion, the present results indicate that the two-sided version of Brown's method is a potentially attractive alternative to the use of the Bonferroni correction and other methods for gene-based testing. It is generally able to control the Type I error rate and can lead to increased power, especially when associations are spread across multiple SNPs and genes. Those are the circumstances characterized by complex diseases where shifting the focus to higher functional structures may in fact be particularly advantageous.

Acknowledgments Parts of this work were carried out on the Dutch national e-infrastructure with the support of SURF Cooperative. 
Chapter 4. A comparison of methods for gene-based testing that account for linkage disequilibrium

\section{References}

[1] T. A. Manolio. "Genomewide Association Studies and Assessment of the Risk of Disease". In: New England Journal of Medicine 363.2 (2010), pp. 166-176. DOI: https://doi.org/10.1056/nejmra0905980.

[2] S. E. Baranzini et al. "Pathway and network-based analysis of genomewide association studies in multiple sclerosis". In: Hum Mol Genet 18.11 (2009), pp. 2078-90. DOI: 10.1093/hmg/ddp120.

[3] S. Jiao et al. "Powerful Set-Based Gene-Environment Interaction Testing Framework for Complex Diseases". In: Genetic Epidemiology 39.8 (2015), pp. 609-618. DOI: https://doi.org/10.1002/gepi.21908.

[4] R. C. Johnson et al. "Accounting for Multiple Comparisons in a GenomeWide Association Study (GWAS)". In: BMC Genomics 11.1 (2010), p. 724. DOI: https://doi.org/10.1186/1471-2164-11-724.

[5] J. M. Bland and D. G. Altman. "Multiple Significance Tests: The Bonferroni Method". In: British Medical Journal 310.6973 (1995), p. 170. DOI: https://doi.org/10.1136/bmj .310.6973.170.

[6] J. P. Shaffer. "Multiple Hypothesis Testing". In: Annual Review of Psychology 46 (1995), pp. 561-584. DOI: https://doi .org/10.1146/ annurev.ps.46.020195.003021.

[7] J. Huang et al. "1000 Genomes-Based Imputation Identifies Novel and Refined Associations for the Wellcome Trust Case Control Consortium Phase 1 Data". In: European Journal of Human Genetics 20.7 (2012), pp. 801-805. DOI: https://doi.org/10.1038/ejhg.2012.3.

[8] R. J. Simes. "An Improved Bonferroni Procedure for Multiple Tests of Significance". In: Biometrika 73.3 (1986), pp. 751-754. DOI: https : // doi.org/10.1093/biomet/73.3.751.

[9] Y. Hochberg. "A Sharper Bonferroni Procedure for Multiple Tests of Significance". In: Biometrika 75.4 (1988), pp. 800-802. DOI: https : // doi.org/10.1093/biomet/75.4.800.

[10] S. Holm. "A Simple Sequentially Rejective Multiple Test Procedure". In: Scandinavian Journal of Statistics 6 (1979), pp. 65-70.

[11] G. Hommel. "A Stagewise Rejective Multiple Test Procedure Based on a Modified Bonferroni Test". In: Biometrika 75.2 (1988), pp. 383-386. DOI: https://doi.org/10.1093/biomet/75.2.383. 
[12] Y. Benjamini and Y. Hochberg. "Controlling the False Discovery Rate: A Practical and Powerful Approach to Multiple Testing". In: Journal of the Royal Statistical Society, Series B (Methodological) (1995), pp. 289-300. DOI: https://doi.org/10.1111/j.2517-6161.1995.tb02031.x.

[13] K. N. Conneely and M. Boehnke. "So many correlated tests, so little time! Rapid adjustment of P values for multiple correlated tests". In: Am J Hum Genet 81.6 (2007), pp. 1158-68. DOI: 10.1086/522036.

[14] S. R. Narum. "Beyond Bonferroni: less Conservative Analyses for Conservation Genetics". In: Conservation Genetics 7.5 (2006), pp. 783-787. DOI: https://doi.org/10.1007/s10592-006-9189-7.

[15] B. Lehne, C. M. Lewis, and T. Schlitt. "From SNPs to Genes: disease Association at the Gene Level". In: PLoS One 6.6 (2011), e20133. DOI: https://doi.org/10.1371/journal . pone.0020133.

[16] J. Z. Liu et al. "A Versatile Gene-Based Test for Genome-Wide Association Studies". In: The American Journal of Human Genetics 87.1 (2010), pp. 139-145. DOI: https://doi.org/10.1016/j.ajhg.2010.06.009.

[17] Jaeyoon Chung et al. "Comparison of methods for multivariate genebased association tests for complex diseases using common variants". In: European Journal of Human Genetics (2019), p. 1. DOI: https://doi. org/10.1038/s41431-018-0327-8.

[18] E. S. Pearson. "The Probability Integral Transformation for Testing Goodness of Fit and Combining Independent Tests of Significance". In: Biometrika 30.1/2 (1938), pp. 134-148. DOI: https : // doi .org/10. $2307 / 2332229$.

[19] H. O. Lancaster. "The Combination of Probabilities Arising from Data in Discrete Distributions". In: Biometrika 36.3/4 (1949), pp. 370-382. DOI: https://doi.org/10.1093/biomet/36.3-4.370.

[20] S. A. Stouffer et al. The American Soldier: Adjustment During Army Life (Studies in Social Psychology in World War II. Vol. 1. Princeton: Princeton University Press, 1949.

[21] B. Wilkinson. "A Statistical Consideration in Psychological Research". In: Psychological Bulletin 48.2 (1951), pp. 156-158. DOI: https://doi. org/10.1037/h0059111.

[22] T Liptak. "On the combination of independent tests". In: Magyar Tud Akad Mat Kutato Int Kozl 3 (1958), pp. 171-197. 
Chapter 4. A comparison of methods for gene-based testing that account for linkage disequilibrium

[23] B. J. Becker. "Combining significance levels". In: The handbook of research synthesis. Ed. by H. Cooper and L. V. Hedges. New York: Russell Sage Foundation, 1994, pp. 215-230.

[24] R. A. Fisher. Statistical Methods for Researchers (4th. ed.) Edinburgh, UK: Oliver and Boyd, 1932.

[25] R. C. Littell and J. L Folks. "Asymptotic Optimality of Fisher's Method of Combining Independent Tests". In: Journal of the American Statistical Association 66.336 (1971), pp. 802-806. DOI: https : //doi .org/10 . 1080/01621459.1971.10482347.

[26] R. C. Littell and J. L Folks. "Asymptotic Optimality of Fisher's Method of Combining Independent Tests II". In: Journal of the American Statistical Association 68.341 (1973), pp. 193-194. DoI: https://doi.org/ $10.1080 / 01621459.1973 .10481362$.

[27] M. Slatkin. "Linkage Disequilibrium-Understanding the Evolutionary Past and Mapping the Medical Future". In: Nature Reviews Genetics 9.6 (2008), pp. 477-485. DOI: https://doi.org/10.1038/nrg2361.

[28] V. Moskvina et al. "Evaluation of an Approximation Method for Assessment of Overall Significance of Multiple Dependent Tests in a Genomewide Association Study". In: Genetic Epidemiology 35.8 (2011), pp. 861-866. DOI: https://doi.org/10.1002/gepi. 20636.

[29] M. B. Brown. "400: A Method for Combining Non-Independent, OneSided Tests of Significance". In: Biometrics (1975), pp. 987-992. DOI: https://doi.org/10.2307/2529826.

[30] Hong Zhang et al. "TFisher Tests: Optimal and Adaptive Thresholding for Combining p-Values". In: arXiv preprint arXiv:1801.04309 (2018).

[31] J. M. Cheverud. "A Simple Correction for Multiple Comparisons in Interval Mapping Genome Scans". In: Heredity 87.1 (2001), pp. 52-58. DOI: https://doi.org/10.1046/j.1365-2540.2001.00901.x.

[32] D. R. Nyholt. "A simple correction for multiple testing for single-nucleotide polymorphisms in linkage disequilibrium with each other". In: Am J Hum Genet 74.4 (2004), pp. 765-9. DOI: $10.1086 / 383251$.

[33] J. Li and L. Ji. "Adjusting multiple testing in multilocus analyses using the eigenvalues of a correlation matrix". In: Heredity (Edinb) 95.3 (2005), pp. 221-7. DOI: $10.1038 / \mathrm{sj}$.hdy .6800717. 
[34] X. Gao, J. Starmer, and E. R. Martin. "A multiple testing correction method for genetic association studies using correlated single nucleotide polymorphisms". In: Genet Epidemiol 32.4 (2008), pp. 361-9. DOI: 10 . 1002/gepi. 20310.

[35] N. W. Galwey. "A new measure of the effective number of tests, a practical tool for comparing families of non-independent significance tests". In: Genet Epidemiol 33.7 (2009), pp. 559-68. DOI: 10.1002/gepi. 20408.

[36] D. Y. Lin. "An Efficient Monte Carlo Approach to assessing Statistical Significance in Genomic Studies". In: Bioinformatics 21.6 (2005), pp. 781-787. DOI: https : // doi . org/10 . 1093/bioinformatics / bti053.

[37] M. X. Li et al. "GATES: a rapid and powerful gene-based association test using extended Simes procedure". In: The American Journal of Human Genetics 88.3 (2011), pp. 283-93. DOI: https://doi.org/10.1016/j. ajhg.2011.01.019.

[38] J. Chapman and J. Whittaker. "Analysis of Multiple SNPs in a Candidate Gene or Region". In: Genetic Epidemiology 32.6 (2008), pp. 560566. DOI: https://doi.org/10.1002/gepi. 20330.

[39] S. H. Wen and Z. S. Lu. "Factors Affecting the Effective Number of Tests in Genetic Association Studies: A Comparative Study of Three PCABased Methods". In: Journal of Human Genetics 56.6 (2011), pp. 428435. DOI: https://doi.org/10.1038/jhg.2011.34.

[40] G. Alves and Y. K. Yu. "Accuracy Evaluation of the Unified P-Value from Combining Correlated P-Values". In: PLoS One 9.3 (2014), e91225. DOI: https://doi.org/10.1371/journal . pone.0103662.

[41] N. M. Laird and C. Lange. The Fundamentals of Modern Statistical Genetics. New York: Springer, 2010.

[42] James J Yang et al. "An efficient genome-wide association test for multivariate phenotypes based on the Fisher combination function". In: $B M C$ bioinformatics 17.1 (2016), p. 19.

[43] The International HapMap Consortium. "The International HapMap Project". In: Nature 426.6968 (2003), pp. 789-796. DOI: https ://doi. org/0.1038/nature02168.

[44] J. J. Goeman and A. Solari. "Multiple Hypothesis Testing in Genomics". In: Statistics in Medicine 33.11 (2014), pp. 1946-1978. DOI: https : // doi.org/10.1002/sim.6082. 
Chapter 4. A comparison of methods for gene-based testing that account for linkage disequilibrium

[45] L. H. C. Tippett. The Methods of Statistics. London: Williams \& Norgate, 1931.

[46] O. J. Dunn. "Estimation of the Means of Dependent Variables". In: Annals of Mathematical Statistics 29.4 (1958), pp. 1095-1111. DOI: https: //doi.org/10.1214/aoms/1177706443.

[47] Z. Šidák. "Rectangular Confidence Regions for the Means of Multivariate Normal Distributions". In: Journal of the American Statistical Associations 62.318 (1957), pp. 626-633. DOI: https://doi.org/10.2307/ 2283989.

[48] M. J. Strube. "Combining and Comparing Significance Levels from Nonindependent Hypothesis Tests". In: Psychological Bulletin 97.2 (1985), pp. 334-341. DOI: https://doi .org/10.1037/0033-2909.97.2.334.

[49] Evelien Van Assche et al. "Gene-Based Interaction Analysis Shows GABA Ergic Genes Interacting with Parenting in Adolescent Depressive Symptoms". In: Journal of Child Psychology and Psychiatry 58.12 (2017), pp. 1301-1309. DOI: 10.1111/jcpp. 12766.

[50] L. S. Radloff. "The CES-D scale: A self-report depression scale for research in the general population". In: Applied Psychological Measurement 1.3 (1977), pp. 385-401. DOI: https : / / doi .org/10 . 1177 / 014662167700100306.

[51] Gregory Warnes et al. genetics: Population Genetics. R package version 1.3.8.1. 2013.

[52] Ozan Cinar and Wolfgang Viechtbauer. poolr: Methods for Pooling PValues from (Dependent) Tests. R package version 0.9-6. 2020.

[53] E. Koch, M. Ristroph, and M. Kirkpatrick. "Long Range Linkage Disequilibrium Across the Human Genome". In: PLoS One 8.12 (2013), e80754. DOI: https://doi.org/10.1371/journal.pone.0080754.

[54] R. E. Mills et al. "An Initial Map of Insertion and Deletion (INDEL) Variation in the Human Genome". In: Genome Research 16.9 (2006), pp. 1182-1190. DOI: https://doi.org/10.1101/gr.4565806.

[55] Tim Hubbard et al. "The Ensembl genome database project". In: Nucleic Acids Research 30.1 (2002), pp. 38-41. DOI: https://doi.org/10.1093/ nar/30.1.38.

[56] S. Durinck et al. "BioMart and Bioconductor: A Powerful Link Between Biological Databases and Microarray Data Analysis". In: Bioinformatics 21.16 (2005), pp. 3439-3440. DOI: https : // doi .org/10.1093/ bioinformatics/bti525. 
[57] S. Durinck et al. "Mapping Identifiers for the Integration of Genomic Datasets with the R/Bioconductor Package biomaRt". In: Nature Protocols 4.8 (2009), pp. 1184-1191. DOI: https://doi.org/10.1038/nprot. 2009.97.

[58] Y. Li et al. "MaCH: using Sequence and Genotype Data to Estimate Haplotypes and Unobserved Genotypes". In: Genetic Epidemiology 434.8 (2010), pp. 816-834. DOI: https://doi.org/10.1002/gepi. 20533.

[59] Douglas Bates et al. "Parsimonious mixed models". In: arXiv preprint arXiv:1506.04967 (2015).

[60] A. Ziegler and I. R. König. A Statistical Approach to Genetic Epidemiology: Concepts and Applications, with an E-Learning Platform. 2nd. Weinheim, Germany: Wiley, 2010.

[61] R Core Team. R: A Language and Environment for Statistical Computing. R Foundation for Statistical Computing. Vienna, Austria, 2020.

[62] B. M. Neale and P. C. Sham. "The future of association studies: Genebased analysis and replication". In: The American Journal of Human Genetics 75.3 (2004), pp. 353-362. DOI: https://doi.org/10.1086/ 423901. 
Chapter 4. A comparison of methods for gene-based testing that account for linkage disequilibrium

\section{Supplementary Materials}

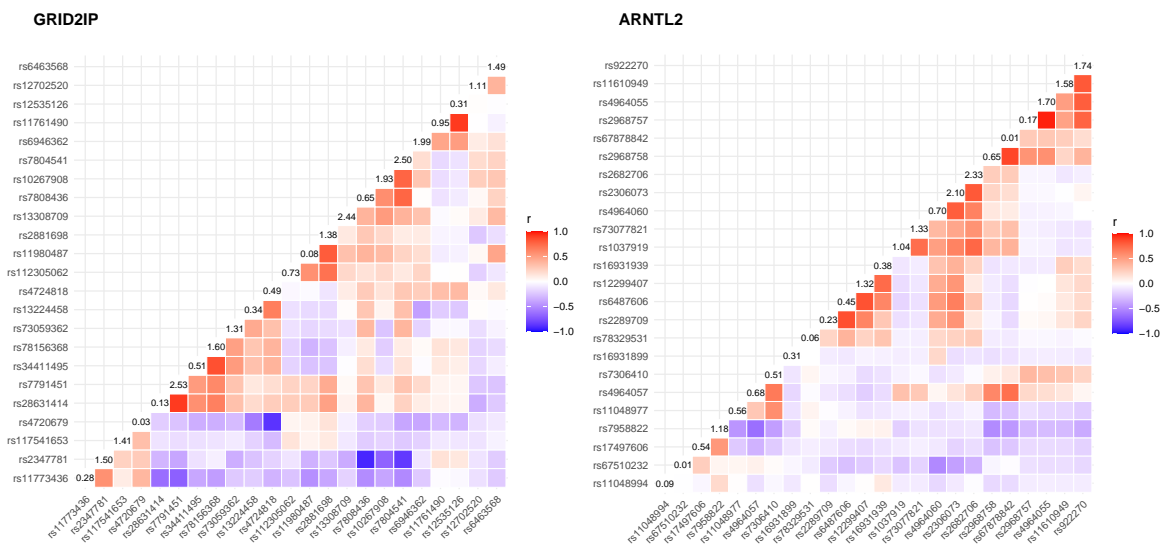

Figure S1: LD maps for the GRID2IP and ARNTL2 genes. The values along the diagonals of the heatmaps show the individual $-\log _{10}(p)$-transformed values of the SNPs. 


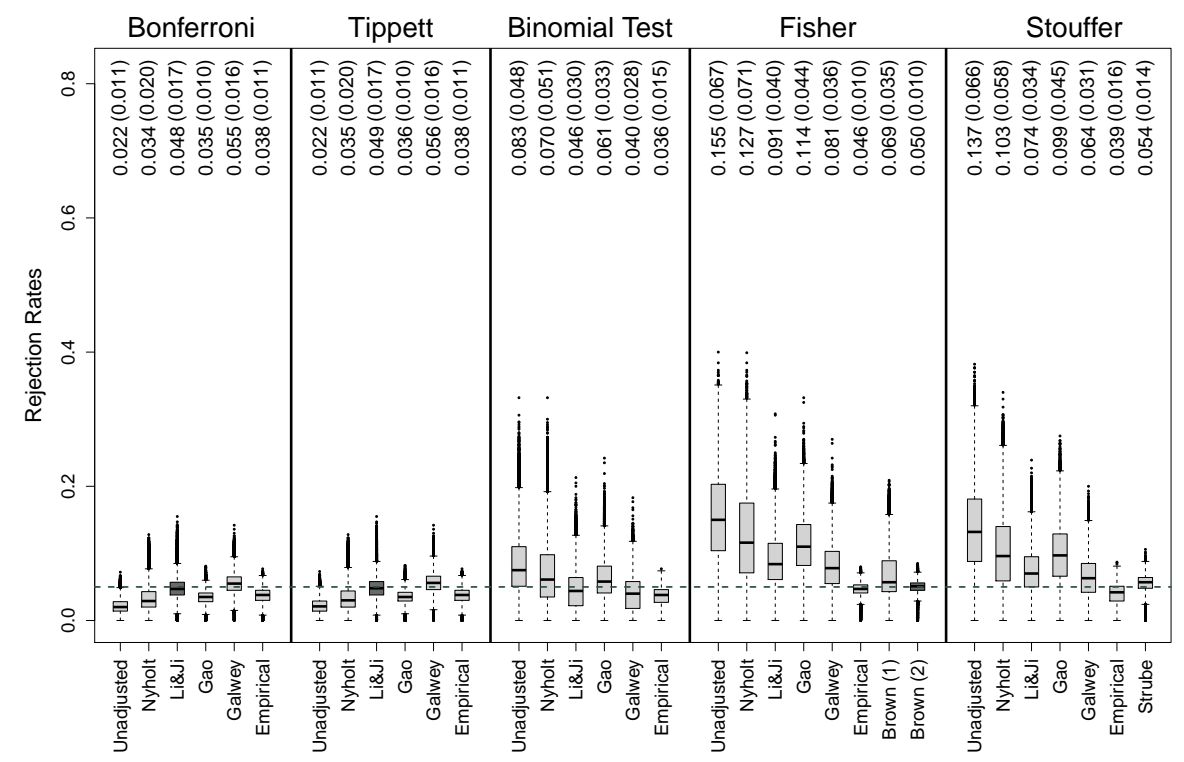

Figure S2: Type I error rates of methods for gene-based testing when applied to the bootstrap samples of sizes 102. The numbers above the boxplots show the mean (SD) rejection rates of the methods. The horizontal grey dashed line corresponds to the nominal rejection rate of $\alpha=0.05$. 
Chapter 4. A comparison of methods for gene-based testing that account for linkage disequilibrium

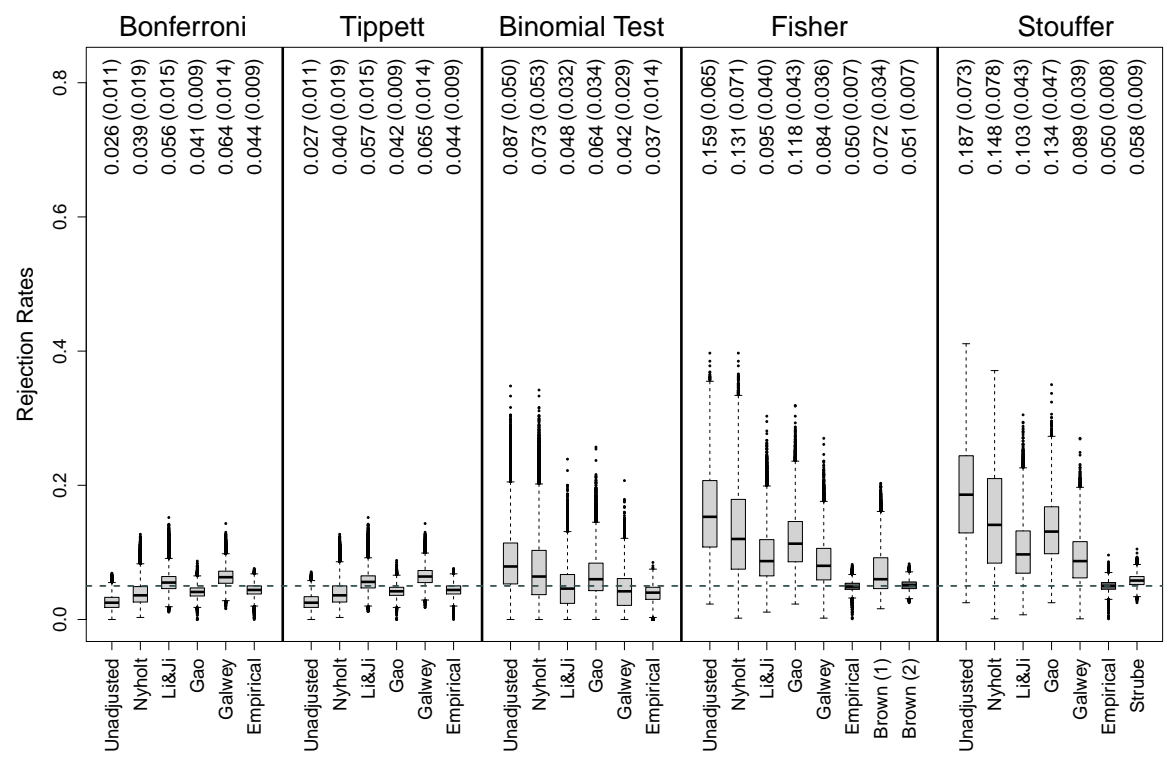

Figure S3: Type I error rates of methods for gene-based testing when applied to the bootstrap samples of sizes 500 . The numbers above the boxplots show the mean (SD) rejection rates of the methods. The horizontal grey dashed line corresponds to the nominal rejection rate of $\alpha=0.05$. 


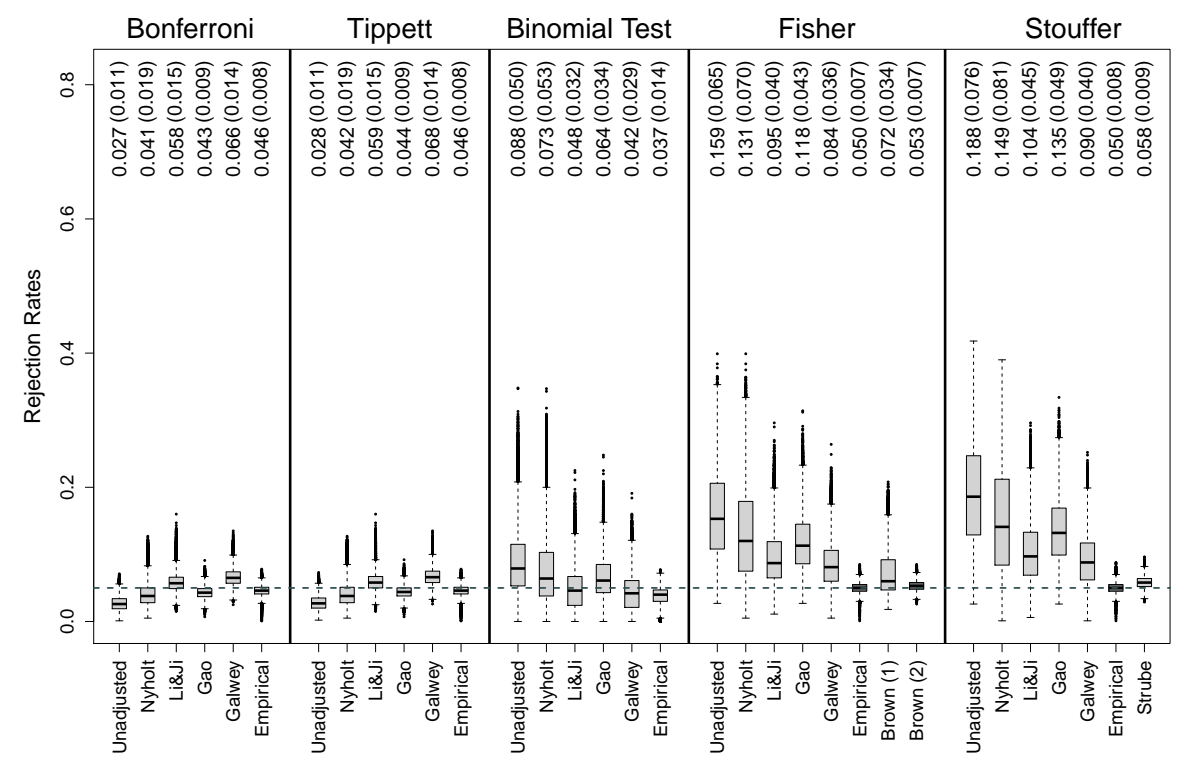

Figure S4: Type I error rates of methods for gene-based testing when applied to the bootstrap samples of sizes 1000 . The numbers above the boxplots show the mean (SD) rejection rates of the methods. The horizontal grey dashed line corresponds to the nominal rejection rate of $\alpha=0.05$. 
Chapter 4. A comparison of methods for gene-based testing that account for linkage disequilibrium
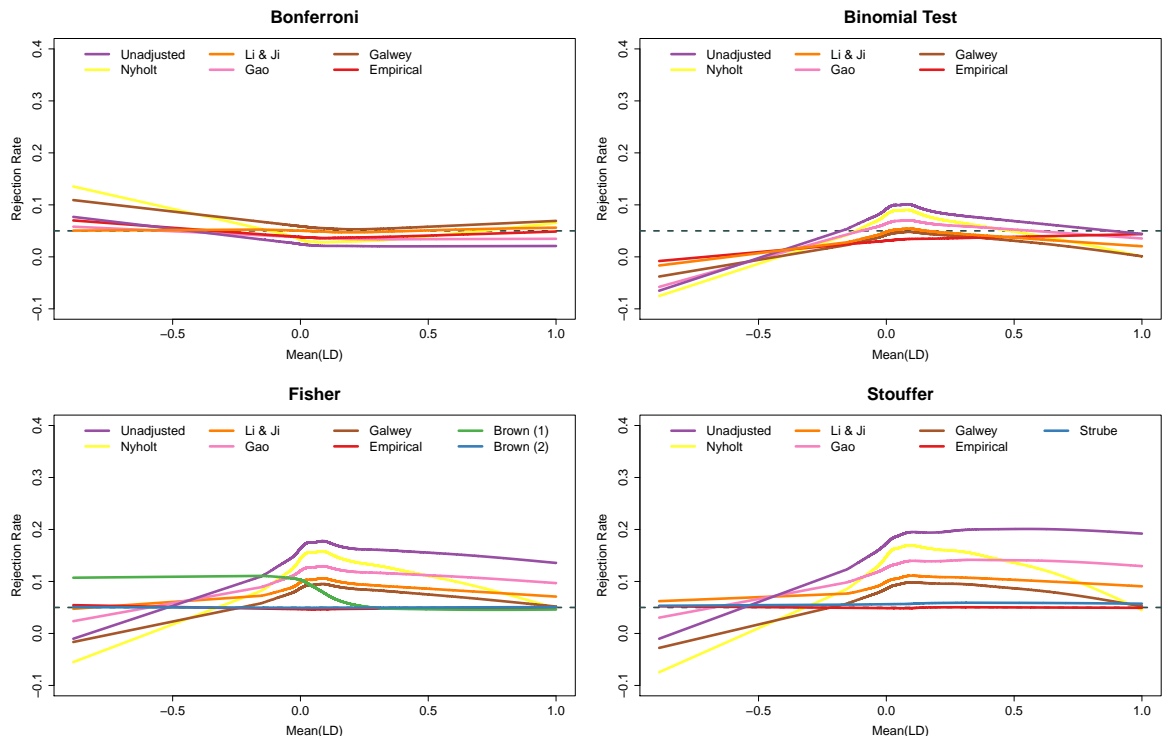

Figure S5: Type I error rates of methods for gene-based testing as a function of the average correlation of the SNPs. The horizontal grey dashed line corresponds to the nominal rejection rate of $\alpha=0.05$. 

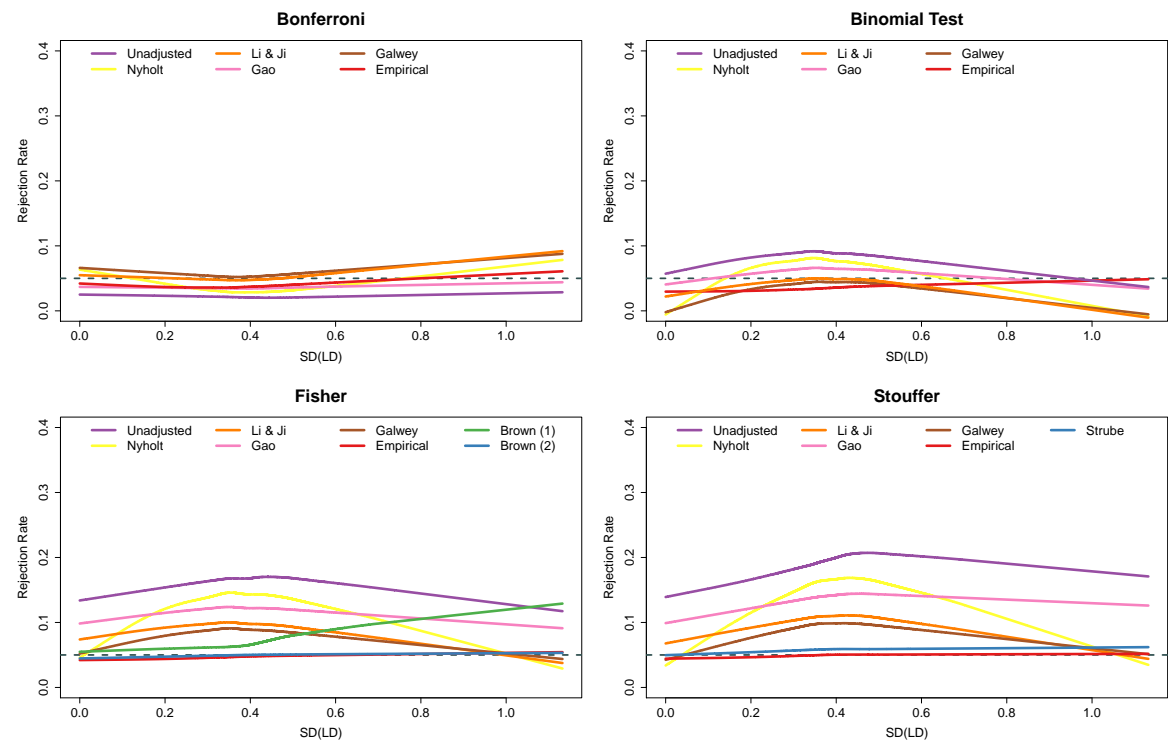

Figure S6: Type I error rates of methods for gene-based testing as a function of the and the standard deviation of the correlations of the SNPs. The horizontal grey dashed line corresponds to the nominal rejection rate of $\alpha=0.05$. 
Chapter 4. A comparison of methods for gene-based testing that account for linkage disequilibrium
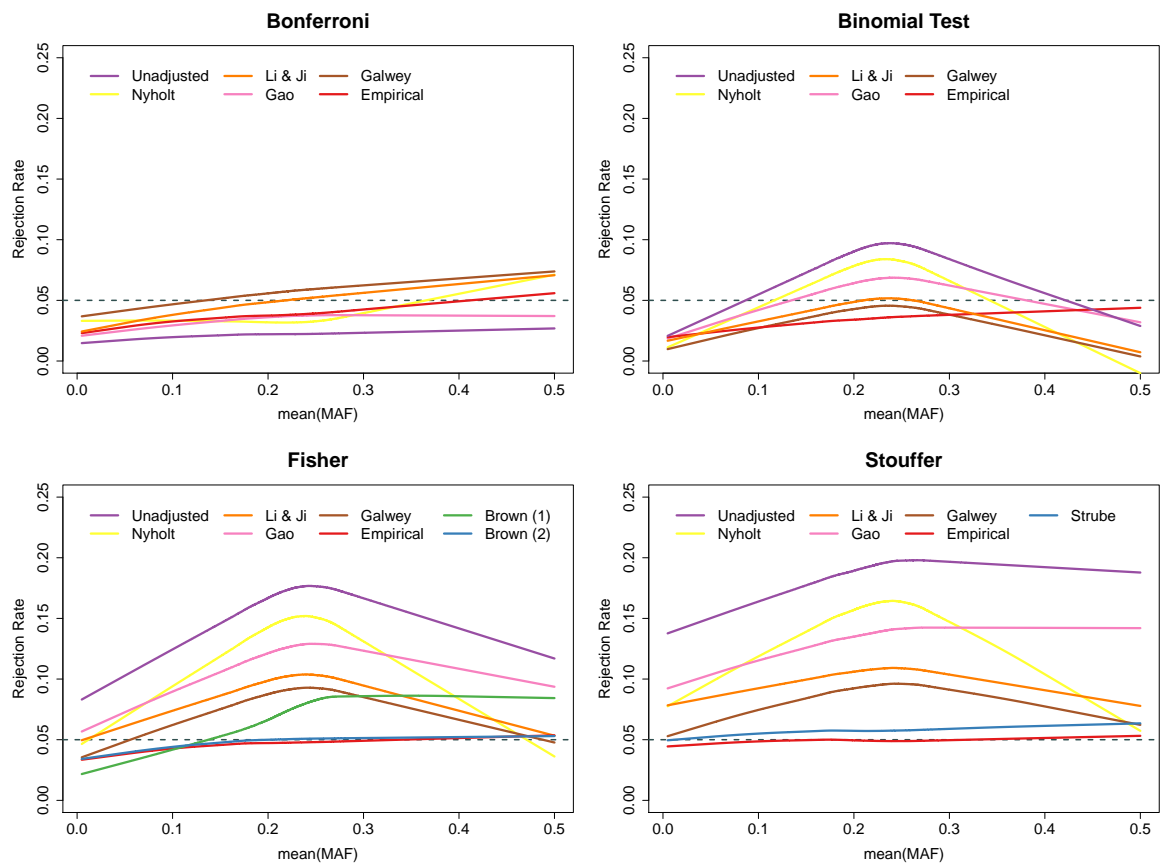

Figure S7: Type I error rates of methods for gene-based testing as a function of the average MAF statistics of the SNPs. The horizontal grey dashed line corresponds to the nominal rejection rate of $\alpha=0.05$. 

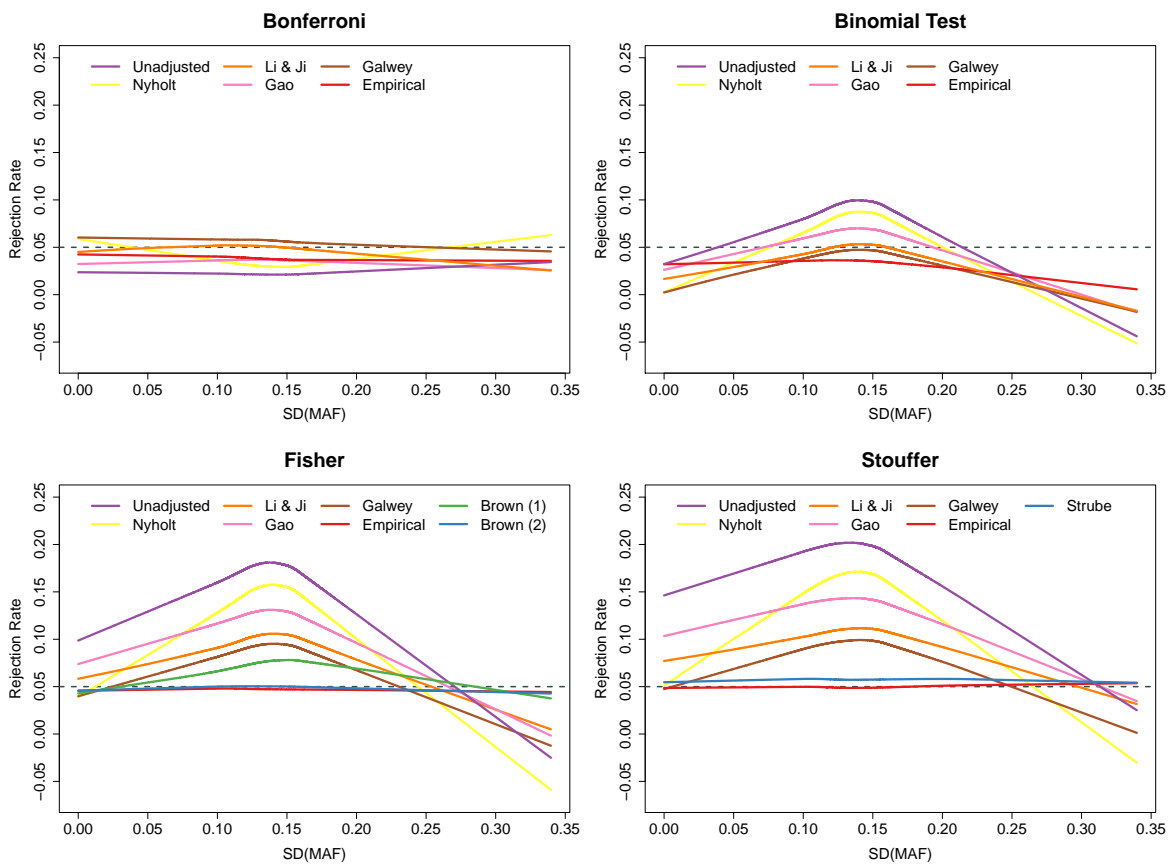

Figure S8: Type I error rates of methods for gene-based testing as a function of the standard deviation of the MAF statistics of the SNPs. The horizontal grey dashed line corresponds to the nominal rejection rate of $\alpha=0.05$. 
Chapter 4. A comparison of methods for gene-based testing that account for linkage disequilibrium

Average Rejection Rates (over $10^{\wedge} 6$ iterations)

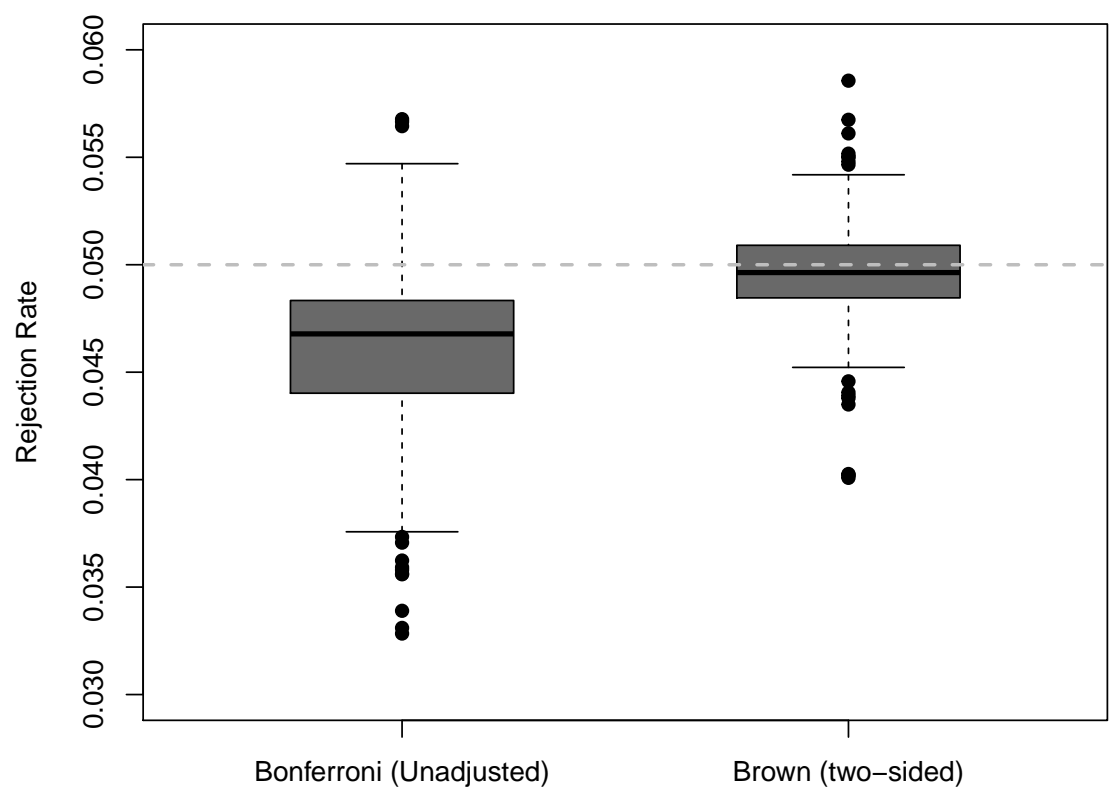

Figure S9: Type I error rates of the Bonferroni and Brown's method applied to 470 genes that had an inflated Type I error rate with the Bonferroni method under the original simulation (with 1,000 iterations) when repeating the simulation using 1,000,000 iterations. 


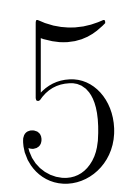

\section{The poolr Package for Combining Independent and Dependent $p$-Values}

Ozan Cinar and Wolfgang Viechtbauer. "The poolr Package for Combining Independent and Dependent $p$-Values". In: ((Being revised for resubmission to) Journal of Statistical Software). 


\section{Abstract}

The poolr package provides an implementation of a variety of methods for pooling (i.e., combining) $p$-values, including Fisher's method, Stouffer's method, the inverse chi-square method, the binomial test, the Bonferroni method, and Tippett's method. More importantly, the methods can be adjusted to account for dependence among the tests from which the $p$-values have been derived. All methods can be adjusted based on an estimate of the effective number of tests or by using an empirically-derived null distribution based on pseudo replicates that mimics a proper permutation test. For the Fisher, Stouffer, and inverse chi-square methods, the test statistics can also be directly generalized to account for dependence, leading to Brown's method, Strube's method, and the generalized inverse chi-square method. In this paper, we describe the various methods, discuss their implementation in the poolr package, illustrate their use based on several examples, and compare the poolr package with several other packages that can be used to combine $p$-values. 


\subsection{Introduction}

"When a number of quite independent tests of significance have been made, it sometimes happens that although few or none can be claimed individually as significant, yet the aggregate gives an impression that the probabilities are on the whole lower than would often have been obtained by chance. It is sometimes desired, taking account only of these probabilities, and not of the detailed composition of the data from which they are derived, which may be of very different kinds, to obtain a single test of the significance of the aggregate, based on the product of the probabilities individually observed." Fisher [1, p. 99]

The quote above, taken from the 4th edition of Fisher's Statistical Methods for Research Workers, reveals a longstanding interest by statisticians and researchers in methods for combining the results of multiple significance tests. The goal is to combine the $p$-values of several significance tests - which are thematically related to each other in some way - into a single $p$-value that tests the significance of the collection as a whole. Numerous methods for this purpose have been described in the literature [for some reviews, see 2, 3, 4].

Some well-known methods include those described by Fisher [1], Stouffer et al. [5], and Wilkinson [6]. However, all of these methods assume that the $p$-values to be combined are derived from independent tests. This assumption is known to be violated in many situations. For example, in genome-wide association studies (GWAS), a million or more single-nucleotide polymorphisms (SNPs) are nowadays tested for their association with some phenotype of interest. The $p$-values from SNPs belonging to the same gene or biological pathway can be combined to shift the focus of the study to higher biological structures [7]. However, SNPs show non-random associations known as linkage disequilibrium (LD) [8], leading to dependence among the tests and hence $p$-values. Ignoring this dependence when combining the $p$-values could lead to overly conservative or to overly liberal Type I error rates [9].

Methods for combining $p$-values can be modified to account for the dependence structure among the tests. For example, Brown [10] proposed using a Satterthwaite approximation for the distribution of the test statistic of Fisher's method that takes the degree of dependence into consideration. Stouffer's method can also be easily generalized to dependent tests [11]. Furthermore, several authors $[12,13,14,15,16]$ have described methods to estimate the effective number of independent tests using principal component analysis (PCA) of a correlation 
matrix that reflects the dependence among the tests. Methods that assume independence can then be modified based on such an estimate to take the degree of dependence into consideration. Finally, it is also possible to combine dependent tests using empirically-derived null distributions using resampling procedures $[17,18]$. Although the latter is often considered a gold-standard technique, especially in the field of genomics, the increased computational burden is an important drawback [19].

At the moment, there are various options available in $\mathrm{R}$ for combining $p$-values of independent and dependent tests. A large number of methods for combining independent $p$-values are implemented in the metap package [20]. Three of these methods (Fisher's, Stouffer's, and the logit-transformation method by [21]) are also available via the combine.test() function of the survcomp package [22], while the aggregation package [23] also provides Fisher's method in addition to the methods by Tippett [24] and Lancaster [25]. Independent $p$-values can also be combined with the Fisher and Stouffer methods using the metap () function of the gap package [26].

For dependent tests, EmpiricalBrownsMethod [27] and CombinePValue [28] provide generalizations of Fisher's method analogous to Brown [10], but avoid the numerical integration required by Brown's method in various ways. However, both implementations require access to the raw data, which limits their applicability to scenarios where only the $p$-values and some kind of correlation matrix reflective of the dependence structure is available. The TFisher package also provides an implementation of Brown's method, and can work directly with a set of $p$-values and a corresponding correlation matrix, but the accompanying manuscript [29] does not provide any technical details about this aspect of the package. Finally, a method for combining (possibly dependent) $p$-values was recently described by Wilson [30], which is implemented in the harmonicmeanp package. We will examine all of these alternative packages in more detail further below.

The primary aim of this paper, however, is to present a new package called poolr, which provides a variety of methods for combining independent, and more importantly, dependent $p$-values. The package contains six 'base methods' which can be adjusted based on four different PCA-based approaches that estimate the effective number of tests. Furthermore, the package can generate empirical null distributions for the base methods to calculate a combined $p$-value that accounts for the dependence structure. Finally, the package includes implementations of Brown's method, Strube's generalization of the Stouffer method, and the generalized inverse chi-square method. 
The paper is structured as follows. In Section 5.2, we describe the methods that are implemented in poolr. The functions and their arguments are then explained in Section 5.3. An illustrative application of the poolr package is provided in Section 5.4, followed by a comparison, in Section 5.5, of the methods implemented in poolr with those in packages that also deal with the combination of the results of independent and dependent tests. Finally, Section 5.6 concludes the paper with a discussion of some miscellaneous topics.

\subsection{Methods}

The problem to be addressed can be succinctly stated as follows. Let $p_{i}$ denote the one- or two-sided $p$-value corresponding to the $i$ th null hypothesis, $H_{0 i}$, where $i=1, \ldots, k$. We assume that the tests being used to generate the $p_{i}$ values have nominal properties (i.e., the Type I error rates are equal to the chosen $\alpha$ value). Therefore, we assume that $p_{i} \sim \operatorname{Uniform}(0,1)$ when $H_{0 i}$ is true. The goal is to combine the $p$-values into a single $p$-value to test the joint null hypothesis that $H_{0 i}$ is true for all $k$ tests (i.e., $H_{0}: \cap_{i=1}^{k} H_{0 i}$ ) against the alternative $H_{1}$ : at least one $H_{0 i}$ is false. We will denote the combined $p$-value with $p_{c}$ and use $\alpha_{c}$ to denote the desired Type I error rate to which $p_{c}$ is compared to determine whether the joint null should be rejected or not.

\subsubsection{Base methods}

Six 'base methods' are available in poolr for this task. All but one of these methods assume that the $p$-values to be combined are independent of each other. These base methods can be adjusted to consider the degree of dependence among the tests. We will start by introducing the base methods and then continue with describing the possible adjustments.

\section{Fisher's method}

Fisher's method [1] is one of the most commonly used techniques for combining a set of $p$-values. Assuming that $p_{i} \sim \operatorname{Uniform}(0,1)$, it can be shown that $-2 \ln \left(p_{i}\right)$ is chi-square distributed with 2 degrees of freedom (df). A combined test statistic can then be calculated with

$$
X^{2}=-2 \sum_{i=1}^{k} \ln \left(p_{i}\right) .
$$


Since the sum of independent chi-square distributed random variables is also chi-square distributed (with df equal to the sum of the df values of the summands), $X^{2}$ follows a chi-square distribution with $\mathrm{df}=2 k$ under the joint null hypothesis. Therefore, the combined $p$-value is given by $p_{c}=1-F\left(X^{2}, 2 k\right)$ where $F(\cdot, f)$ denotes the cumulative distribution function of a chi-square distribution with $\mathrm{df}=f$.

\section{Stouffer's method}

Stouffer's method [5] is another well-known method for pooling $p$-values. Let $\Phi(\cdot)$ denote the cumulative distribution function of a standard normal distribution and $\Phi^{-1}(\cdot)$ its inverse. Given that the $p$-values are uniformly distributed under the null hypothesis, $z_{i}=\Phi^{-1}\left(1-p_{i}\right)$ follows a standard normal distribution, and thus

$$
z=\sum_{i=1}^{k} z_{i} / \sqrt{k}
$$

is also standard normal under the joint null. Note that we use $1-p_{i}$ in the computation of $z_{i}$ such that this will yield a large positive value of $z_{i}$ when $p_{i}$ is small. The combined $p$-value can then be calculated with $p_{c}=1-\Phi(z)$.

\section{Inverse chi-square method}

Analogous to Stouffer's 'inverse-normal' method, in the inverse chi-square method (not to be confused with Fisher's method) we transform the $p$-values using the inverse of the cumulative distribution function of a chi-square distribution with one degree of freedom, which we denote by $F^{-1}(\cdot, 1)$. Hence

$$
X^{2}=\sum_{i=1}^{k} F^{-1}\left(1-p_{i}, 1\right)
$$

follows a chi-square distribution with $\mathrm{df}=k$ and the combined $p$-value is then given by $p_{c}=1-F\left(X^{2}, k\right)$. 


\section{Binomial test}

The binomial test [6] follows naturally after noting that rejection versus nonrejection of a true null hypothesis can be considered to be a Bernoulli distributed random variable with the rejection probability denoted by $\alpha$. Hence, when all $k$ null hypotheses are true, the number of tests that lead to rejection, say $r$, follows a Binomial distribution, that is, $r \sim \operatorname{Binomial}(k, \alpha)$. Therefore, we can compute the combined $p$-value with

$$
p_{c}=\sum_{x=r}^{k}\left(\begin{array}{l}
k \\
x
\end{array}\right) \alpha^{x}(1-\alpha)^{k-x},
$$

which gives the probability of obtaining $r$ or more rejections ('successes') under the joint null. Hence, if $p_{c} \leq \alpha_{c}$, we reject the joint null. We can therefore regard the binomial test as a method for examining whether there is an 'excess of significant results' among the set of $k$ tests when assuming that the joint null hypothesis is true.

Note that $\alpha$ (i.e., the threshold for declaring an individual test as significant or not) can be different from $\alpha_{c}$ (the desired Type I error rate for the combined test). In fact, due to the discrete nature of $p_{c}$ as computed with (5.4), the Type I error rate of the binomial test is bounded by $\alpha_{c}$ from above, that is, its actual Type I error rate may be lower than $\alpha_{c}$ (i.e., $\operatorname{Pr}\left(p_{c} \leq \alpha_{c}\right) \leq \alpha_{c}$ for the binomial test, with strict equality holding for the Fisher, Stouffer, and inverse chi-square methods). ${ }^{1}$

\section{Bonferroni method}

The last two methods to be described are more commonly thought of as multiple testing correction techniques, but are equally applicable in the present context. The first is the well-known Bonferroni correction [e.g., 31], arguably one of the most commonly applied multiple testing correction techniques in research. As used for that purpose, the hypothesis-wise Type I error rate (i.e., $\alpha$ ) is divided by the number of simultaneous tests (i.e., $k$ ). Then $\alpha / k$ is used

\footnotetext{
${ }^{1}$ Also note that when $\alpha_{c}<\alpha^{k}$, we can never reject the joint null with the binomial test. This may be a non-issue when testing a single joint null hypothesis, but in some situations (e.g., genetics), we often test many joint null hypotheses (e.g., for multiple genes), in which case $\alpha_{c}$ may be set to a low value to correct for multiple testing. It may then be impossible to reject a particular joint null hypothesis for which $k$ is too low.
} 
as the significance threshold for the individual tests, which can be shown to control the family-wise Type I error rate (i.e., the probability of committing at least one Type I error across all $k$ tests is then at most $\alpha$ ). Equivalently, we can multiply the $p$-value of each test by $k$ and use $\alpha$ as the significance threshold (i.e., tests for which $p_{i} \times k \leq \alpha$ are significant).

Although the Bonferroni method is typically formulated as a multiple testing correction, it is also possible to use it as a method for combining $p$-values. The motivation is that the joint null hypothesis should be rejected if at least one of the individual null hypotheses is rejected [cf. 32]. For this, we only need to consider the smallest $p$-value of the tests whose $p$-values are to be combined. Therefore, the combined $p$-value is then computed with

$$
p_{c}=\min \left(1, \min \left(p_{1}, \ldots, p_{k}\right) \times k\right),
$$

which can be compared with $\alpha_{c}$ to test the joint null hypothesis.

In contrast to the previous methods (and contrary to common belief), the Bonferroni method does not assume independence among the tests [31]. Hence, it controls the Type I error rate (in the sense that $\operatorname{Pr}\left(p_{c} \leq \alpha_{c}\right) \leq \alpha_{c}$ under the joint null) whether the tests are independent or not. However, the method is slightly conservative under independence (e.g., for $\alpha_{c}=.05$, the actual Type I error rate converges to $\left.\lim _{k \rightarrow \infty} 1-(1-.05 / k)^{k}=1-e^{-.05} \approx 0.0488\right)$ and it can be much more conservative when the tests are dependent.

The use of the Bonferroni correction as a method for combining $p$-values shows that all multiple testing correction methods (that directly operate on the $p$ values) can also be used as methods for combining $p$-values. As long as one of the corrected $p$-values leads to a rejection, the joint null can also be rejected. Therefore, one could consider other multiple testing correction methods that are less conservative than the Bonferroni method. The one that immediately comes to mind is Holm's method [33], which is also valid under any type of dependence structure among the tests and is less conservative than the Bonferroni correction [31].

Holm's procedure is a sequential 'step-down' variant of the Bonferroni correction. It starts by multiplying the smallest $p$-value with the number of simultaneous tests. If this corrected $p$-value is larger than $\alpha$, then none of the null hypotheses are rejected, whereas testing continues with the second smallest $p$-value if the first step does lead to a rejection (and so on). As a method for combining $p$-values (i.e., for testing the joint null hypothesis), Holm's proce- 
dure is therefore identical to the Bonferroni method, since only the smallest $p$-value (multiplied by $k$ ) needs to be considered.

Other multiple testing correction methods such as those described by Simes [32], Hochberg [34], Hommel [35], Benjamini and Hochberg [36], and Benjamini and Yekutieli [37] can also be used as methods for combining $p$-values in the same manner and, compared to the Bonferroni/Holm method, can potentially lead to different conclusions when testing a joint null hypothesis. However, the methods by Benjamini and Hochberg [36] and Benjamini and Yekutieli [37] are not meant to control the family-wise Type I error rate, but the false discovery rate, and the other methods will only control the family-wise Type I error rate under independence or, in some cases, under the assumption of 'positive dependence through stochastic ordering' (PDS) [31].

\section{Tippett's method}

Tippett's method (also known as the Dunn-Šidák correction, based on [38] and [39]) is another multiple testing correction [24]. As used for this purpose, the hypothesis-wise error rate is set to $1-(1-\alpha)^{1 / k}$, which controls the family-wise Type I error rate at $\alpha$ (i.e., it does not exhibit the slightly conservative behavior of the Bonferroni method), but is only guaranteed to do so under independence. The joint null hypothesis would therefore be rejected if $\min \left(p_{i}\right) \leq 1-(1-\alpha)^{1 / k}$. Correspondingly, the combined $p$-value based on Tippett's method is given by

$$
p_{c}=1-\left(1-\min \left(p_{1}, \ldots, p_{k}\right)\right)^{k},
$$

which should be compared with $\alpha_{c}$ to test the significance of the joint null hypothesis.

\subsubsection{Adjustments}

In the previous sections, we described six base methods for combining $p$-values that are available in the poolr package. Five of these methods are only guaranteed to control the Type I error rate when the $p$-values to be combined are independent. The Bonferroni correction is the only exception as it does not make assumptions about the dependence structure among the $p$-values. However, its Type I error rate can be quite conservative when the tests are dependent. Therefore, for all methods, we can apply certain adjustments that may help to bring their Type I error rates closer to the nominal level even when the tests are dependent. 
For these adjustments, we assume that the $p$-values to be combined are derived from $k$ hypothesis tests with test statistics $t_{1}, \ldots, t_{k}$ that follow, under the joint null hypothesis, a multivariate normal distribution with means equal to zero, variances equal to unity, and correlation matrix $\mathrm{R}_{t}$. For example, suppose we conduct $k$ one-sample z-tests of the null hypotheses $H_{0 i}: \mu_{i}=\mu_{0 i}$ for $i=1, \ldots, k$ based on $n$ observations of the random variables $X_{1}, X_{2}, \ldots, X_{k}$, which themselves are multivariate normal. Then the scenario outlined above is exactly fulfilled since the joint null distribution of the test statistics is then also multivariate normal. Moreover, the correlations among the $k$ random variables to be tested are exactly identical to the correlations among the test statistics of the z-tests.

The same scenario arises in other contexts, at least asymptotically. For example, when conducting one- or two-sample t-tests with interchanging variables, the test statistics converge in distribution to multivariate normality. The same applies when fitting $k$ regression models with interchanging variables (each testing the association between some predictor and one of $k$ different response variables, or vice-versa, when testing the association between one of $k$ different predictors and some response variable). In fact, under the regularity conditions of the multivariate central limit theorem [e.g., 40], the interchanging variables do not have to follow a multivariate normal distribution for the test statistics to converge to multivariate normality. Hence, the scenario covers not only linear but also other types of regression models (e.g., logistic, Poisson). Moreover, while $\mathrm{R}_{t}$ is not exactly known in any of these examples, the correlations among the interchanging variables can be collected in a $k \times k$ correlation matrix denoted by $\mathrm{R}_{x}$, which is a consistent estimate of $\mathrm{R}_{t}$ (in which case the multidimensional central limit theorem still holds; [41]).

Hence, in what follows, we assume that the dependence among the tests and hence $p$-values arises through the correlations among a set of interchanging elements across the analyses, as reflected by the correlation matrix $\mathrm{R}_{x}$. The latter can be computed from the data at hand or from reference databases in some fields [18].

\section{Effective number of tests}

When conducting $k$ tests that are dependent in the manner described above, we can think of this as a scenario where we are effectively conducting a smaller number of independent tests. For example, suppose three SNPs are tested for their association with some phenotype of interest. If the SNPs are in perfect 
linkage equilibrium (and hence there is no correlation among the genotypes at the three locations), we are conducting three independent tests. On the other hand, if the SNPs are in perfect LD, then we are actually conducting the same test three times, leading to three identical $p$-values, and hence the effective number of tests is one. Depending on the degree of LD among the SNPs, the effective number of tests will therefore lie somewhere between these two extremes. A variety of methods have been proposed based on this concept to quantify the degree of dependence among multiple tests.

All of these methods start by applying principal component analysis (PCA) to $\mathrm{R}_{x}$, the matrix with the correlations among the interchanging elements $[12$, $16,15,14,13]$. Let $\lambda_{i}$ denote the $i$ th eigenvalue thereof such that $\lambda_{1} \geq \lambda_{2} \geq$ $\ldots \geq \lambda_{k}$. Then Cheverud [12] and Nyholt [13] propose to estimate the effective number of tests with

$$
m_{C N}=1+(k-1)\left(1-\frac{\operatorname{Var}(\lambda)}{k}\right),
$$

where $\operatorname{Var}(\lambda)$ is the observed sample variance among the $k$ eigenvalues. Alternatively, Li and $\mathrm{Ji}[14]$ suggest to calculate the effective number of tests with

$$
m_{L J}=\sum_{i=1}^{k} h\left(\left|\lambda_{i}\right|\right),
$$

where $h(x)=I(x \geq 1)+(x-\lfloor x\rfloor)$ and $\lfloor\cdot\rfloor$ is the floor function. Another approach, described by Gao, Starmer, and Martin [15], estimates the effective number of tests with

$$
m_{G A O}=\underset{x \in\{1, \ldots, k\}}{\operatorname{argmin}}\left(\frac{\sum_{i=1}^{x} \lambda_{i}}{\sum_{i=1}^{k} \lambda_{i}}>C\right),
$$

where $C$ is a user-defined parameter which is usually set to 0.995 (i.e., $m_{G A O}$ is the number of principal components needed such that $C \times 100 \%$ of the total variance is accounted for). Finally, according to Galwey [16], the effective number of tests should be computed with

$$
m_{G A L}=\frac{\left(\sum_{i=1}^{k} \sqrt{\lambda_{i}}\right)^{2}}{\sum_{i=1}^{k} \lambda_{i}} .
$$

Although on first sight the calculations appear to be rather different, the meth- 
ods have some common properties. When $\mathrm{R}_{x}$ is an identity matrix, then $\lambda_{i}=1$ for $i=1, \ldots, k$ and hence $m=k$ (where $m$ denotes one of the estimates of the effective number of tests described above). The only exception to this is $m_{G A O}$, as it may yield an estimate less than $k$, depending on $C$ and $k$ (e.g., when $C=0.995$ and $k>200$, then $m_{G A O}<k$ ). On the other hand, when all correlations in $\mathrm{R}_{x}$ are equal to 1 , then $\lambda_{1}=k$ and $\lambda_{i}=0$ for $i=2, \ldots, k$ and hence $m=1$ for all methods. However, differences in the value of $m$ can arise for intermediate cases, as we will illustrate later on.

Once $m$ is calculated with one of the methods described above, the methods that were described in Section 5.2.1 can be adjusted based on this quantity. In particular, the test statistic for Fisher's method is then computed with

$$
\tilde{X}^{2}=\frac{m}{k} \times X^{2},
$$

where $X^{2}$ is given by (5.1). Then $\tilde{X}^{2}$ is assumed to follow a chi-square distribution with $\mathrm{df}=2 \mathrm{~m}$ and hence the combined $p$-value is now calculated with $p_{c}=1-F\left(\tilde{X}^{2}, 2 m\right)$. Similarly, for the inverse chi-square method, we also use (5.11), except that $X^{2}$ is then given by (5.3) and the combined $p$-value is calculated with $p_{c}=1-F\left(\tilde{X}^{2}, m\right)$. The test statistic for Stouffer's method is adjusted with

$$
\tilde{z}=\sqrt{\frac{m}{k}} \times z,
$$

where $z$ is given by (5.2). The combined $p$-value is then calculated with $p_{c}=$ $1-\Phi(\tilde{z})$. For the binomial method, we first compute $\tilde{r}=\left\lfloor\frac{r \times m}{k}\right\rfloor$ and then obtain the combined $p$-value with

$$
p_{c}=\sum_{x=\tilde{r}}^{m}\left(\begin{array}{c}
m \\
x
\end{array}\right) \alpha^{x}(1-\alpha)^{m-x} .
$$

Instead of using the floor function, one could also compute $\tilde{r}$ using rounding, but we prefer the former for its conservativeness. Finally, for the Bonferroni and Tippett methods, we simply replace $k$ with $m$ and hence the combined $p$-values are calculated with

$$
p_{c}=\min \left(1, \min \left(p_{1}, \ldots, p_{k}\right) \times m\right)
$$

and

$$
p_{c}=1-\left(1-\min \left(p_{1}, \ldots, p_{k}\right)\right)^{m} \text {, }
$$


respectively.

The adjustments are made in such a way so that they simplify to the base methods when $m=k$. Moreover, when $m=1$, the same test is effectively conducted $k$ times, leading to $k$ identical $p$-values (i.e., $p \equiv p_{1}=\ldots=p_{k}$ ). In this case, the 'combined' $p$-value based on the adjusted methods will be identical to $p$, except for the binomial method, which either yields a $p$-value of 1 (when $p>\alpha$, so that $\tilde{r}=0$ ) or equal to $\alpha$ (when $p \leq \alpha$, so that $\tilde{r}=1$ ).

\section{Correlation among dependent $p$-values}

Although PCA is typically applied to $\mathrm{R}_{x}$, one can make the argument that this matrix is not the most direct reflection of the dependence structure among the $p$-values. In addition, one also needs to consider the type and sidedness of the tests conducted. For example, suppose for $n$ observations of the random variables $X_{1}, X_{2}, \ldots, X_{k}$, we conduct $k$ one-sample t-tests of the null hypotheses $H_{0 i}: \mu_{i}=\mu_{0 i}$ for $i=1, \ldots, k$. When conducting two-sided tests, then simulations conducted by the authors indicate that the correlations among the $p$-values are roughly approximated by $\left|\mathrm{R}_{x}\right|^{3}$ under the joint null hypothesis. On the other hand, when testing one-sided, the correlations among the $p$-values are approximately given by $\mathrm{R}_{x}$. The same approximations also appear to hold when conducting two-sample t-tests for a set of $k$ variables and for regression models (either with a single response variable and $k$ different predictors or vice-versa).

In fact, under the stated assumptions, we can derive the correlations among the $p$-values directly. In particular, assume $\left[t_{i}, t_{j}\right]^{\prime} \sim \operatorname{MVN}\left([0,0]^{\prime},\left[\begin{array}{cc}1 & \rho_{i j} \\ \rho_{i j} & 1\end{array}\right]\right)$, where $\rho_{i j}$ is the element in the $i$ th row and $j$ th column of $\mathrm{R}_{t}$. Making use of the 'law of the unconscious statistician' [42], the correlation among the one-sided $p$-values, $p_{i}=1-\Phi\left(t_{i}\right)$ and $p_{j}=1-\Phi\left(t_{j}\right)$, is then given by

$$
\operatorname{Cor}\left(p_{i}, p_{j}\right)=12 \int_{-\infty}^{+\infty}\left(1-\Phi\left(t_{i}\right)\right) \times\left(1-\Phi\left(t_{j}\right)\right) f\left(t_{i}, t_{j}\right) d t_{i} d t_{j}-3
$$

since $\mathrm{E}\left[p_{i}\right]=\mathrm{E}\left[p_{j}\right]=1 / 2$ and $\operatorname{Var}\left[p_{i}\right]=\operatorname{Var}\left[p_{j}\right]=1 / 12$. For two-sided $p$ values, $p_{i}=2\left(1-\Phi\left(\left|t_{i}\right|\right)\right)$ and $p_{j}=2\left(1-\Phi\left(\left|t_{j}\right|\right)\right)$, the correlation is given 
by

$$
\operatorname{Cor}\left(p_{i}, p_{j}\right)=12 \int_{-\infty}^{+\infty} \int^{+} 2\left(1-\Phi\left(\left|t_{i}\right|\right)\right) \times 2\left(1-\Phi\left(\left|t_{j}\right|\right)\right) f\left(t_{i}, t_{j}\right) d t_{i} d t_{j}-3 .
$$

For example, if $\rho_{i j} \in(0,0.3,0.6,0.9,0.95)$, then $\operatorname{Cor}\left(p_{i}, p_{j}\right)$ is equal to $(0.000$, $0.288,0.582,0.892,0.946)$ for one-sided and $(0.000,0.056,0.250,0.701,0.831)$ for two-sided tests. The approximations given earlier (i.e., $\mathrm{R}_{x}$ and $\left|\mathrm{R}_{x}\right|^{3}$ ) are therefore not far off (i.e., $\left|\rho_{i j}\right|^{3}=0.000,0.027,0.216,0.729,0.857$ ).

Hence, it may be advantageous to construct a matrix based on the correlations among the $p$-values using these 'exact' computations before computing the effective number of tests as described above. For this purpose, poolr provides appropriate lookup tables for (5.16) and (5.17) that obviate the need for the numerical integration described above (which can be time consuming when $k$ is large, since $k(k-1) / 2$ correlations need to be computed).

\section{Empirically-derived null distributions using pseudo replicates}

Another approach to account for the dependence among the $p$-values is to make use of resampling methods such as permutation tests [e.g., 43, 44]. In fact, in certain research areas (e.g., genomics), permutation tests are often regarded as a gold-standard technique $[45,19]$.

The basic idea behind a permutation test is to reshuffle the data in such a way that relevant features of the data structure are preserved (e.g., the correlation among multiple predictors or response variables) except for the association to be tested. Based on the reshuffled data, we then compute the test statistic of interest. By repeating this process a large number of times, an empirical distribution of the test statistic under the null hypothesis is generated. The proportion of values in this empirical distribution that are at least as extreme as the observed test statistic (i.e., the one obtained with the original data) then yields the $p$-value of the permutation test.

As a simple example of how permutation tests can be used to combine $p$-values, suppose we test the association between the case-control status of a group of subjects with each of $k$ SNPs in a gene using the Cochran-Armitage trend test [e.g., 46, 47] with the goal of testing the significance of the gene as a whole. The smallest $p$-value among the $k$ SNPs could then serve as the test statistic 
of interest. For the permutation test, we randomly reshuffle the case-control status of the subjects (preserving the degree of LD among the SNPs), computing the smallest $p$-value of the trend tests for each rearrangement. The permutation-based combined $p$-value of the $k$ tests is then given by the proportion of times that the smallest $p$-value under the permutation distribution is equal to or smaller than the smallest observed $p$-value (i.e., closer to 0 is more 'extreme' for this test statistic).

Using the smallest $p$-value as the test statistic of interest is a common approach in permutation testing [e.g., 46, 47]. It is easy to see that this is equivalent to using either the Bonferroni or Tippett's method as the test statistic of interest (for the permutation test, the two methods yield an identical combined $p$ value, since they are both monotonic functions of $\left.\min \left(p_{1}, \ldots, p_{k}\right)\right)$. However, since no single method for combining $p$-values is optimal (i.e., none of the methods is a uniformly most powerful test; [48]), one could also consider using the combined $p$-value from one of the other base methods as the test statistic of interest (e.g., for GWAS, [19] actually recommend to base the permutation procedure on Fisher's method).

Permutation-based methods are attractive, as they can be regarded as nonparametric, valid under broad assumptions, and in some sense 'exact' [49]. If implemented correctly, the test also automatically reflects relevant properties of the data such as the correlations among the predictors or response variables [50]. An important drawback is the computational burden of the method. An exact permutation test requires computing the test statistic of interest under every possible rearrangement of the data. However, the number of rearrangement is often so large as to make this computationally infeasible. Instead, a large number of random rearrangements are typically used. Regardless, to obtain a stable approximation of the $p$-value that would have been obtained with an exact permutation test, we need to generate the test statistic under a large number of random rearrangements, which can still be a substantial computational burden [19], especially when permutation tests need to be repeatedly conducted. For example, in GWAS, one might easily test 20,000 or more genes in this manner. Unless heavily parallelized, these computations can take days or even weeks to complete. Moreover, since the $p$-values for the genes would usually be corrected for multiple testing, it is necessary to estimate tail-area $p$-values accurately, possibly requiring $10^{6}-10^{7}$ iterations per gene (e.g., using a Bonferroni correction for multiple testing in 20,000 genes, the difference between $.00001 \times 20,000=0.2$ and $.000001 \times 20,000=0.02$ is highly relevant). 
We can greatly speed up this process by using an empirically-derived null distribution that is not based on the original data, but by using pseudo replicates [e.g., 17, 18]. The general logic of this procedure is as follows. As described earlier, we assume that the joint null distribution of the test statistics of the $k$ tests that were conducted can be approximated by a multivariate normal distribution with a mean vector of zeros and that $\mathrm{R}_{x}$ (the matrix with the correlations among the interchanging elements across the tests) serves as an estimate of the variance-covariance matrix of these test statistics. Hence, let $\mathrm{Z}$ denote an $(s \times k)$ matrix of $s$ replicates of random data generated from such a $k$-dimensional multivariate normal distribution and either $\mathrm{P}=1-\Phi(\mathrm{Z})$ or $\mathrm{P}=2 \times(1-\Phi(|\mathrm{Z}|))$ - depending on whether one- or two-sided tests were originally conducted - the matrix with the corresponding $p$-values, with $\Phi(\cdot)$ applied element-wise. An empirically-derived null distribution can then be obtained by applying a particular base method to the values in each row of $\mathrm{P}$, yielding $p_{c}^{1}, \ldots, p_{c}^{s}$. The combined $p$-value is then given by

$$
p_{c}=\frac{\sum_{l=1}^{s} \mathrm{I}\left(p_{c}^{l} \leq p_{c}^{\mathrm{obs}}\right)+1}{s+1}
$$

where $p_{c}^{\text {obs }}$ is the combined $p$-value obtained from the observed $p$-values and $\mathrm{I}(\cdot)$ is the indicator function. We add 1 in the numerator and denominator to avoid the possibility of obtaining a combined $p$-value equal to zero and to yield a test with the correct size [51].

In comparison to a 'proper' permutation test, this approach requires only a fraction of the computation time. A further advantage of this method is that it does not require access to the raw data, only the $p$-values of the individual tests and the correlation matrix $\mathrm{R}_{x}$ (and in some situations, the latter can be approximated based on reference datasets or other external information).

\section{Generalized methods derived under dependence}

Finally, it is also possible to modify the Fisher, Stouffer, and inverse chisquare methods so that the dependence structure is directly considered in the construction of the respective test statistic and the calculation of the combined $p$-value. The first method is based on Brown [10], who described a modification of Fisher's method for pooling the results of $k$ one-sided one-sample z-tests of the null hypotheses $H_{0 i}$ : $\mu_{i} \leq \mu_{0 i}$ for the random variables $X_{1}, X_{2}, \ldots, X_{k}$ that are assumed to have a joint multivariate normal distribution. This is in fact an example of the scenario outlined earlier, since the joint null distribution of 
the test statistics is then also multivariate normal and the correlations among the $k$ random variables to be tested are exactly identical to the correlations among the test statistics of the z-tests (i.e., $\mathrm{R}_{x}=\mathrm{R}_{t}$ ).

Under the joint null hypothesis, the $X^{2}$ value of Fisher's method then has expected value $E\left(X^{2}\right)=2 k$ and variance $\operatorname{Var}\left(X^{2}\right)=4 k+2 \sum_{i=1}^{k-1} \sum_{j>i}^{k} \operatorname{Cov}\left(-2 \ln \left(p_{i}\right),-2 \ln \left(p_{j}\right)\right)$, where the covariance term is given by

$$
\begin{aligned}
\operatorname{Cov}(-2 & \left.\ln \left(p_{i}\right),-2 \ln \left(p_{j}\right)\right)= \\
& \\
& 4 \int_{-\infty}^{+\infty} \int_{\infty} \ln \left(1-\Phi\left(t_{i}\right)\right) \times \ln \left(1-\Phi\left(t_{j}\right)\right) f\left(t_{i}, t_{j}\right) d t_{i} d t_{j}-4,
\end{aligned}
$$

which can be computed using numerical integration under the stated assumptions. Brown [10] then proposed to use a Satterthwaite approximation to the distribution of $X^{2}$ based on a scaled chi-square distribution, that is, assume that $X^{2} \sim c \chi_{f}^{2}$ (or equivalently, $X^{2} / c \sim \chi_{f}^{2}$ ), where $\chi_{f}^{2}$ is a chi-square random variable with $\mathrm{df}=f$. In that case, $X^{2}$ has expected value $c f$ and variance $2 f c^{2}$ and we can equate these quantities to $E\left(X^{2}\right)$ and $\operatorname{Var}\left(X^{2}\right)$, respectively. Solving these equations for $c$ and $f$ yields $f=2\left(\mathrm{E}\left(X^{2}\right)\right)^{2} / \operatorname{Var}\left(X^{2}\right)$ and $c=\operatorname{Var}\left(X^{2}\right) /\left(2 \mathrm{E}\left(X^{2}\right)\right)$. Following this, the combined $p$-value can then be calculated with $p_{c}=1-F\left(X^{2} / c, f\right)$.

As noted by Yang et al. [52], for two-sided $p$-values, the only change required is that we compute the covariance with

$$
\begin{aligned}
\operatorname{Cov}( & \left.-2 \ln \left(p_{i}\right),-2 \ln \left(p_{j}\right)\right)= \\
\quad & \\
& +\int_{-\infty}^{+\infty} \int_{\infty} \ln \left(2\left(1-\Phi\left(\left|t_{i}\right|\right)\right)\right) \times \ln \left(2\left(1-\Phi\left(\left|t_{j}\right|\right)\right)\right) f\left(t_{i}, t_{j}\right) d t_{i} d t_{j}-4 .
\end{aligned}
$$

The rest of the procedure remains unchanged. While Brown [10], Kost and McDermott [53], and Yang et al. [52] have provided closed-form approximations to (5.19) and (5.20) to avoid the numerical integration step, the poolr package makes use of lookup tables for (5.19) and (5.20) that were precomputed using Gaussian quadrature to high precision (see below for details). In this way, we provide an 'exact' implementation of Brown's method that is computationally efficient.

The next base method whose test statistic can be directly calculated under 
dependence is Stouffer's method, which was generalized to dependent tests by Strube [11]. For this, we compute the combined test statistic with

$$
z=\frac{\sum_{i=1}^{k} z_{i}}{\sqrt{\operatorname{Var}\left(\sum_{i=1}^{k} z_{i}\right)}}
$$

and $p_{c}=1-\Phi(z)$ as before. The difficulty then lies in computing the denominator, since $\operatorname{Var}\left(\sum_{i=1}^{k} z_{i}\right)=k+2 \sum_{i=1}^{k-1} \sum_{j>i}^{k} \operatorname{Cov}\left(\Phi^{-1}\left(1-p_{i}\right), \Phi^{-1}(1-\right.$ $\left.\left.p_{j}\right)\right)$. For one-sided tests, Strube [11] suggested to approximate $\operatorname{Cov}\left(\Phi^{-1}(1-\right.$ $\left.\left.p_{i}\right), \Phi^{-1}\left(1-p_{j}\right)\right)$ by $\rho_{i j}$, which follows directly from the scenario outlined earlier, since

$$
\begin{aligned}
& \operatorname{Cov}\left(\Phi^{-1}\left(1-p_{i}\right), \Phi^{-1}\left(1-p_{j}\right)\right)= \\
& \quad \int_{-\infty}^{+\infty} \int^{-1}\left(1-\left(1-\Phi\left(t_{i}\right)\right)\right) \times \\
& \quad \Phi^{-1}\left(1-\left(1-\Phi\left(t_{j}\right)\right)\right) f\left(t_{i}, t_{j}\right) d t_{i} d t_{j}=\rho_{i j} .
\end{aligned}
$$

For two-sided tests, the covariance is then given by

$$
\begin{gathered}
\operatorname{Cov}\left(\Phi^{-1}\left(1-p_{i}\right), \Phi^{-1}\left(1-p_{j}\right)\right)= \\
\quad \iint_{-\infty}^{+\infty} \Phi^{-1}\left(1-2\left(1-\Phi\left(\left|t_{i}\right|\right)\right)\right) \times \\
\quad \Phi^{-1}\left(1-2\left(1-\Phi\left(\left|t_{j}\right|\right)\right)\right) f\left(t_{i}, t_{j}\right) d t_{i} d t_{j}
\end{gathered}
$$

which can be evaluated using numerical integration.

However, two aspects of Strube's method when applied to two-sided tests require further attention. First, (5.23) is an improper integral with an infinite discontinuity when either $t_{i}$ or $t_{j}$ is equal to 0 , which raises the concern that (5.23) is divergent. Although we cannot prove this analytically, we can however demonstrate via simulation methods that (5.23) converges to a finite and fixed value and hence (5.23) must be convergent. ${ }^{2}$ Second, Strube's method

\footnotetext{
${ }^{2}$ For this, we generate a large number of random $\left(t_{i}, t_{j}\right)$ pairs from a bivariate normal distribution with known correlation, transform these values into $z_{i}$ and $z_{j}$, and then compute their covariance. For an increasing number of samples, the covariance between $z_{i}$ and $z_{j}$ converges to a finite and fixed value that is identical to the covariance derived via numerical integration of (5.23).
} 
makes the implicit assumption that the $z_{i}$ values are multivariate normal, so that the numerator in (5.21) is also normal. For one-sided tests, this is unobjectionable, at least for the assumed scenario where the test statistics are multivariate normal (in which case the transformation of the one-sided $p$-values to the $z_{i}$ values simply yields back the original test statistics). However, for two-sided tests, the $z_{i}$ values are not multivariate normal (although each $z_{i}$ is marginally normal), even when the test statistics were so to begin with. As a result, the numerator of (5.21) and hence $z$ is not normal. Asymptotically (i.e., for sufficient large $k$ ), normality of $z$ may still hold, but demonstrating this for particular cases requires further assumptions underlying versions of the central limit theorem for dependent random variables [e.g., 54, 55]. For example, for tests based on data with a spatial or temporal configuration, this would require that the degree of dependence decays with the distance between the data points at a sufficiently fast rate. However, characterizing the specific conditions under which the asymptotic normality of $z$ can be safely assumed would require further work. For these reasons, we would caution against the use of Strube's method for combining the results of two-sided tests at the moment.

As an alternative, one can generalize the inverse chi-square method in an analogous manner as was done by Brown for Fisher's method. In particular, for the test statistic (5.3), it generally holds that $E\left(X^{2}\right)=k$ and $\operatorname{Var}\left(X^{2}\right)=2 k+2 \sum_{i=1}^{k-1} \sum_{j>i}^{k} \operatorname{Cov}\left(F^{-1}\left(1-p_{i}, 1\right), F^{-1}\left(1-p_{j}, 1\right)\right)$ under the joint null hypothesis. For one- and two-sided tests, the covariance is given by

$$
\begin{aligned}
& \operatorname{Cov}\left(F^{-1}\left(1-p_{i}, 1\right), F^{-1}\left(1-p_{j}, 1\right)\right)= \\
& \quad \iint_{-\infty}^{+\infty} F^{-1}\left(1-\left(1-\Phi\left(t_{i}\right)\right), 1\right) \\
& \quad \times F^{-1}\left(1-\left(1-\Phi\left(t_{j}\right)\right), 1\right) f\left(t_{i}, t_{j}\right) d t_{i} d t_{j}-1
\end{aligned}
$$


and

$$
\begin{aligned}
& \operatorname{Cov}\left(F^{-1}\left(1-p_{i}, 1\right), F^{-1}\left(1-p_{j}, 1\right)\right)= \\
& \quad \iint_{-\infty}^{+\infty} F^{-1}\left(1-2\left(1-\Phi\left(\left|t_{i}\right|\right)\right), 1\right) \times \\
& \quad F^{-1}\left(1-2\left(1-\Phi\left(\left|t_{j}\right|\right)\right), 1\right) f\left(t_{i}, t_{j}\right) d t_{i} d t_{j}-1,
\end{aligned}
$$

respectively, which we can again evaluate numerically under the assumption of multivariate normality of the original test statistics. As in Brown's method, we use a Satterthwaite approximation with $f=2\left(\mathrm{E}\left(X^{2}\right)\right)^{2} / \operatorname{Var}\left(X^{2}\right)$ and $c=$ $\operatorname{Var}\left(X^{2}\right) /\left(2 \mathrm{E}\left(X^{2}\right)\right)$ and then compute the combined $p$-value with $p_{c}=1-$ $F\left(X^{2} / c, f\right)$.

\subsection{Implementation}

This section describes the functions in the poolr package which can be used to apply the methods presented in the previous section. The development version of the package is hosted on GitHub (https://github.com/ozancinar/poolr) and can be installed via the remotes package and then loaded as follows. ${ }^{3}$

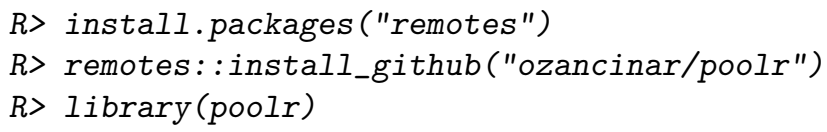

The poolr package is primarily used via six functions that implement the 'base methods' described in Section 5.2.1, namely:

- fisher(): for Fisher's method,

- stouffer(): for Stouffer's method,

- invchisq(): for the inverse chi-square method,

- binotest (): for the binomial test,

- bonferroni(): for the Bonferroni method,

\footnotetext{
${ }^{3}$ The package is also available via CRAN and can be installed in the usual manner (i.e., install.packages("poolr")), but we focus here on the development version available via GitHub, as it incorporates a few (minor) updates. The CRAN version will eventually updated accordingly.
} 
- tippett (): for Tippett's method.

The functions feature a consistent syntax. The following are the arguments of the fisher () function as an illustration.

$R>\operatorname{args}($ fisher)

function ( $\mathrm{p}$, adjust $=$ "none", $\mathrm{R}, \mathrm{m}$, size $=10000$, threshold, side $=2$, batchsize, nearpd $=$ TRUE, $\ldots$ )

NULL

We will explain the purpose of the various arguments in the following sections.

\subsubsection{Combining independent $p$-values}

Independent $p$-values can be combined by supplying a vector of $p$-values to the $\mathrm{p}$ argument of the base functions. For example:

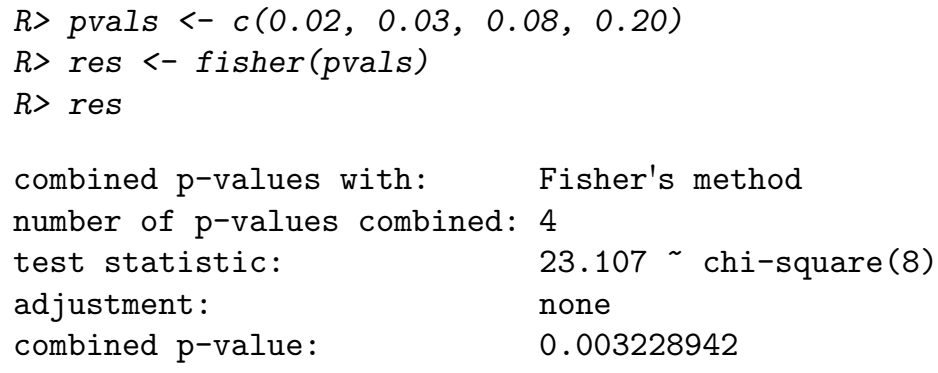

The results are stored as a list in an object of class poolr that is printed with the S3 method print.poolr(). The output displays the combined $p$-value along with additional information about the methods used. The information shown in the third line varies with respect to the base function. The fisher(), stouffer(), and invchisq() functions display the respective test statistic and its assumed distribution under the joint null from which the combined $p$-value is derived, whereas the output of the binotest () function shows the number of significant individual $p$-values. ${ }^{4}$ The remaining functions, bonferroni() and tippett(), simply indicate the minimum individual $p$-value. If only the

\footnotetext{
${ }^{4}$ For binotest (), the default value of $\alpha$ (i.e., the significance level of the individual tests) is .05. A different value of $\alpha$ can be passed to the function via the ... argument (e.g., binotest $(p$, alpha $=.01)$ ).
} 
combined $p$-value is of interest, it is stored as list element $\mathrm{p}$. Hence, for these toy data, the combined $p$-value for all six methods can be obtained with:

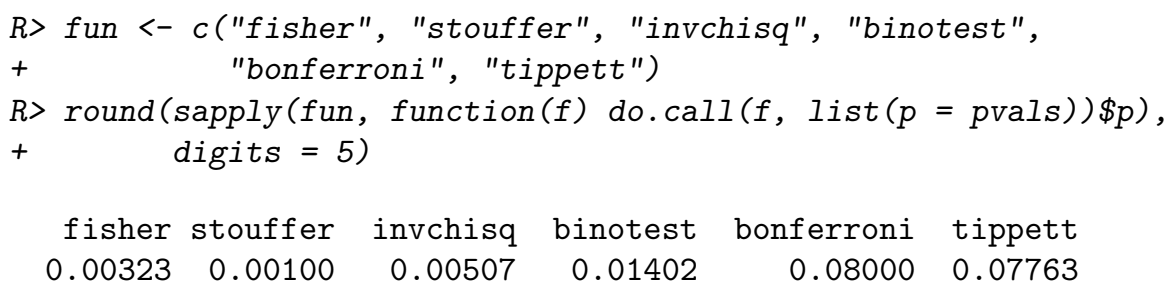

As the example shows, conclusions can differ (e.g., for $\alpha_{c}=.05$ ) depending on the method used for combining the $p$-values.

Note that one should not conclude based on this example that the Bonferroni and Tippett methods are always less powerful than the other methods. Among other things, the power of the methods depends on whether the 'signal' is concentrated in one or a few tests or distributed across all or many of them. In the former case, the Bonferroni and Tippett methods will tend to be more powerful, which we can illustrate with a simple simulation where we repeatedly generate normally distributed test statistics with mean 0 for all but one of $k$ tests, turn these test statistics into (two-sided) $p$-values, apply each method, and obtain its empirical rejection rate:

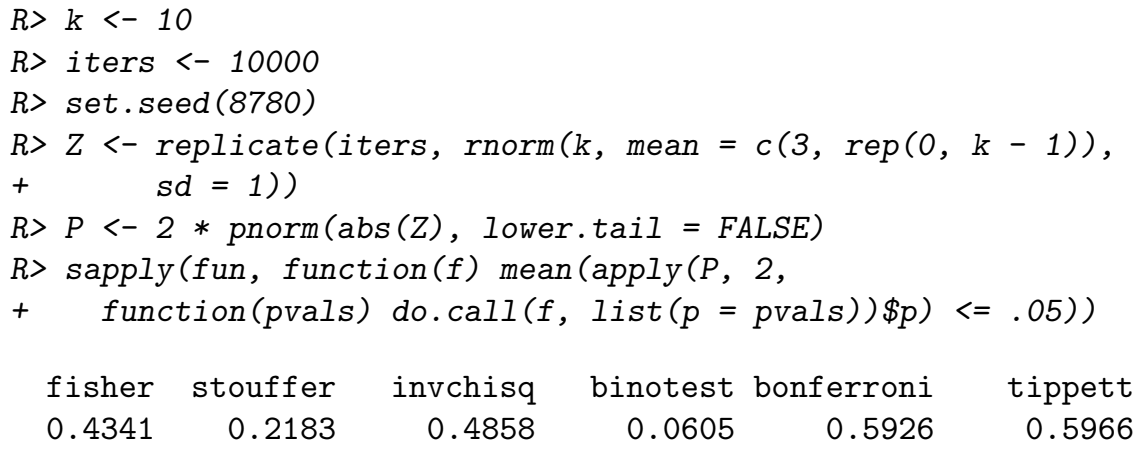

On the other hand, when the null hypothesis is false for all of the tests, then Fisher's, Stouffer's, and the inverse chi-square method will tend to have higher power:

$R>Z<-\operatorname{replicate}($ iters, $\operatorname{rnorm}(k, \operatorname{mean}=\operatorname{rep}(1, k), s d=1)$ )

$R>P<-2 *$ pnorm (abs $(Z)$, lower.tail = FALSE) 


$$
\begin{aligned}
& R>\text { sapply(fun, function(f) mean(apply( } P, 2 \text {, } \\
& +\quad \text { function(pvals) do.call }(f, \operatorname{list}(p=p \text { vals })) \$ p)<=.05) \text { ) } \\
& \text { fisher stouffer invchisq binotest bonferroni tippett } \\
& \begin{array}{llllll}
0.5549 & 0.5114 & 0.5467 & 0.2393 & 0.3006 & 0.3053
\end{array}
\end{aligned}
$$

\subsubsection{Combining dependent $p$-values}

Under dependence, the base functions can be modified using the adjustment techniques described in Section 5.2.2. In the following sections, we will demonstrate the use of these techniques on a set of correlated $p$-values generated below. We will assume that there are $k=5$ correlated tests and the test statistics derived from these tests follow a multivariate normal distribution with zero means and unit variances (i.e., the joint null hypothesis is true) and that the test statistics have a constant correlation of 0.7 .

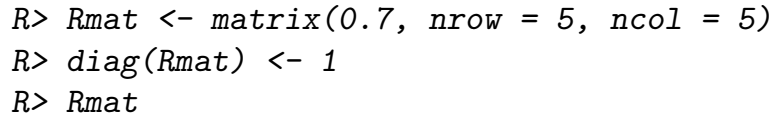

$\begin{array}{lrrrrr} & {[, 1]} & {[, 2]} & {[, 3]} & {[, 4]} & {[, 5]} \\ {[1,]} & 1.0 & 0.7 & 0.7 & 0.7 & 0.7 \\ {[2,]} & 0.7 & 1.0 & 0.7 & 0.7 & 0.7 \\ {[3,]} & 0.7 & 0.7 & 1.0 & 0.7 & 0.7 \\ {[4,]} & 0.7 & 0.7 & 0.7 & 1.0 & 0.7 \\ {[5,]} & 0.7 & 0.7 & 0.7 & 0.7 & 1.0\end{array}$

We can then simulate the test statistics and corresponding (two-sided) $p$-values with:

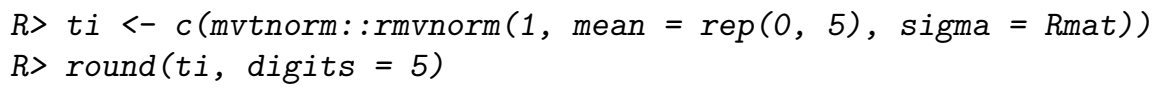

Coincidentally, when (incorrectly) assuming independence, all methods except the ones by Bonferroni and Tippett yield a significant combined $p$-value for these data: 


$$
\begin{aligned}
& R>\operatorname{round}(\operatorname{sapply}(f u n, \text { function }(f) \operatorname{do} \operatorname{call}(f, \text { list }(p=p v a l s)) \$ p) \text {, } \\
& + \text { digits }=5 \text { ) } \\
& \text { fisher stouffer invchisq binotest bonferroni tippett } \\
& \begin{array}{llllll}
0.03770 & 0.02142 & 0.04782 & 0.02259 & 0.18416 & 0.17109
\end{array}
\end{aligned}
$$

Adjusting based on the effective number of tests

As a first type of adjustment, we can estimate the effective number of tests $(m)$ and incorporate this information into the methods for combining the $p$-values. For this, we set argument adjust to "nyholt", "liji", "gao", or "galwey" corresponding to (5.7), (5.8), (5.9), and (5.10), respectively. ${ }^{5}$ Argument $\mathrm{R}$ is then used to specify the correlation matrix of the test statistics. The estimate of $m$ is provided in the output along with a reference to the article where the method was first described. Below is an example where we adjust Fisher's method using an estimate of the effective number of tests based on $\mathrm{Li}$ and $\mathrm{Ji}$ [14]:

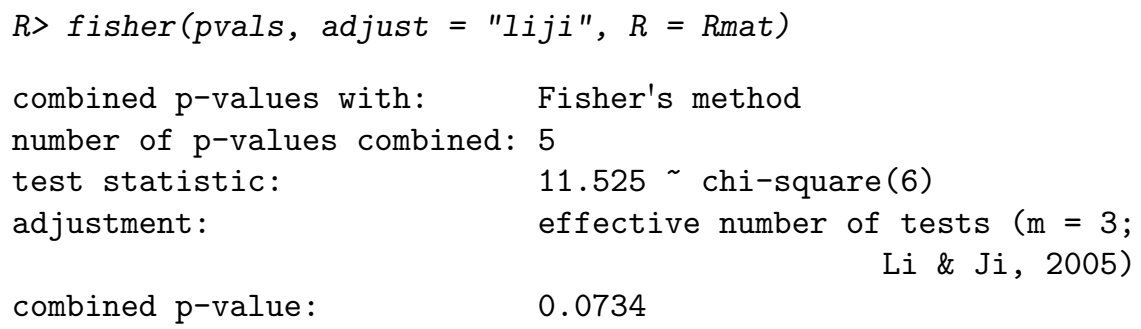

The base functions employ the meff() function of the poolr package to estimate the effective number of tests during this process. This function can also be called directly if only the estimate of $m$ is of interest. For example, we can quickly compare the estimates of $m$ across the different estimation methods with:

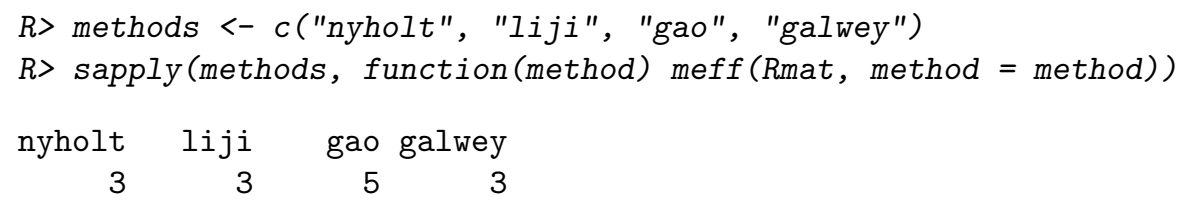

${ }^{5}$ When using the Gao adjustment, $C=0.995$ by default, but a different value of $C$ can be passed to the function via the ... argument (e.g., fisher (pvals, adjust = "gao", R = Rmat, $\mathrm{C}=0.95)$ ). 
All estimates agree here except for the method by Gao, Starmer, and Martin [15], which gives the somewhat surprising estimate of $m=k$.

For clarity, we should note that Rmat is treated here synonymously as the correlation matrix among the test statistics, $\mathrm{R}_{t}$, and as the correlation matrix of the interchanging elements across the five tests, $\mathrm{R}_{x}$ (e.g., when running five regression models, each testing the association between some predictor of interest and one of five different response variables). Assuming that the size of the sample (based on which the test statistics were obtained) was sufficiently large, the distinction between these two correlation matrices is negligible. However, this will not always be true and we will consider in more detail the relationship between these two types of correlation matrices in the discussion section.

Furthermore, in the example above, we used the correlation matrix among the test statistics $\left(\mathrm{R}_{t}\right)$ for estimating $m$. Earlier, we discussed the possibility of using the correlations among the $p$-values instead. For the multivariate normal case, the mvnconv() function can be used to obtain these correlations (based on equations 5.16 and 5.17).

The function has arguments mvnconv $(R$, side $=2$, target, cov2cor $=$ FALSE). The correlation matrix of the test statistics is passed to the function via the $R$ argument, while argument side specifies the sidedness of the tests (i.e., 1 or 2 for one- or two-sided tests, respectively). The target argument is a character string to specify for which target statistic the covariance matrix should be obtained, and logical argument cov2cor can be set to TRUE if the covariance matrix should be converted into the corresponding correlation matrix. For the present purposes, we would like to convert Rmat into the correlation matrix of the two-sided $p$-values (based on equation 5.17), so we set target $=$ "p" and cov2cor = TRUE:

$R>$ mvnconv(Rmat, target $=" p ", \operatorname{cov} 2 \operatorname{cor}=$ TRUE $)$

$$
[, 1] \quad[, 2] \quad[, 3] \quad[, 4] \quad[, 5]
$$

$[1,] \quad \begin{array}{lllllll}1.0000000 & 0.3589436 & 0.3589436 & 0.3589436 & 0.3589436\end{array}$

$\left[\begin{array}{llllllll}{[2,]} & 0.3589436 & 1.0000000 & 0.3589436 & 0.3589436 & 0.3589436\end{array}\right.$

$\left[\begin{array}{llllllll}{[3,]} & 0.3589436 & 0.3589436 & 1.0000000 & 0.3589436 & 0.3589436\end{array}\right.$

$\left[\begin{array}{llllllll}4,] & 0.3589436 & 0.3589436 & 0.3589436 & 1.0000000 & 0.3589436\end{array}\right.$

$\left[\begin{array}{llllllll}{[5,]} & 0.3589436 & 0.3589436 & 0.3589436 & 0.3589436 & 1.0000000\end{array}\right.$

The resulting correlation matrix can then be used in combination with any of the base functions and methods for estimating the effective number of tests. For example: 


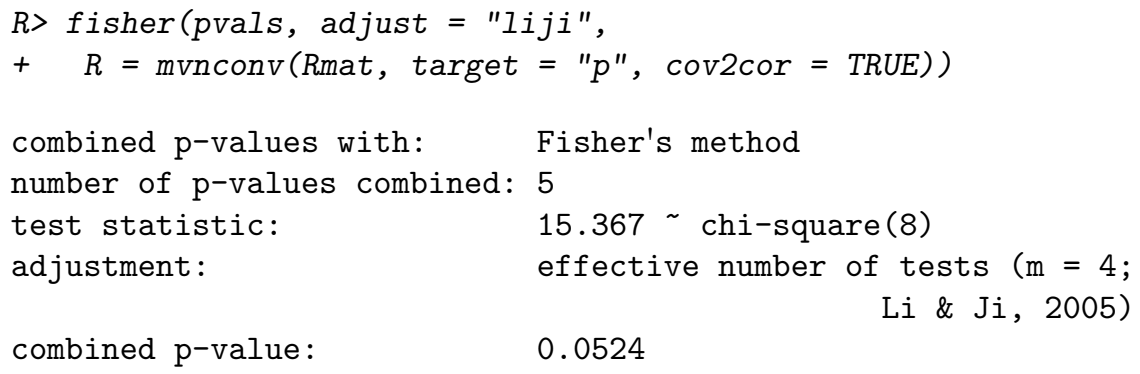

Finally, to provide additional flexibility, the base functions also have an argument called $\mathrm{m}$ which allows the user to specify an estimate of the effective number of tests (e.g., as obtained with some other method not implemented in the poolr package). Returning to the earlier example where we used Rmat directly as input, these two approaches are therefore synonymous (output omitted):

$R>$ fisher (pvals, adjust = "liji", $R=$ Rmat)

$R>$ fisher (pvals, $m=\operatorname{meff}($ Rmat, method $=$ "liji"))

Adjusting based on empirically-derived null distributions

When setting adjust = "empirical", the combined $p$-value is obtained via empirically-derived null distributions as described earlier. Argument $\mathrm{R}$ is then used to specify the correlation matrix of the test statistics, while argument size specifies the size of the null distribution that should be generated $(s=$ 10,000 by default). The latter is done by the empirical () function, which uses (a slightly simplified version of) mvtnorm: :rmvnorm() to generate the $s \times k$ matrix of pseudo replicates of the test statistics, converts them to one- or twosided $p$-values (depending on the side argument, which is set to 2 by default), and then applies the respective base method to each row. For example, we can apply this approach to Fisher's method with:

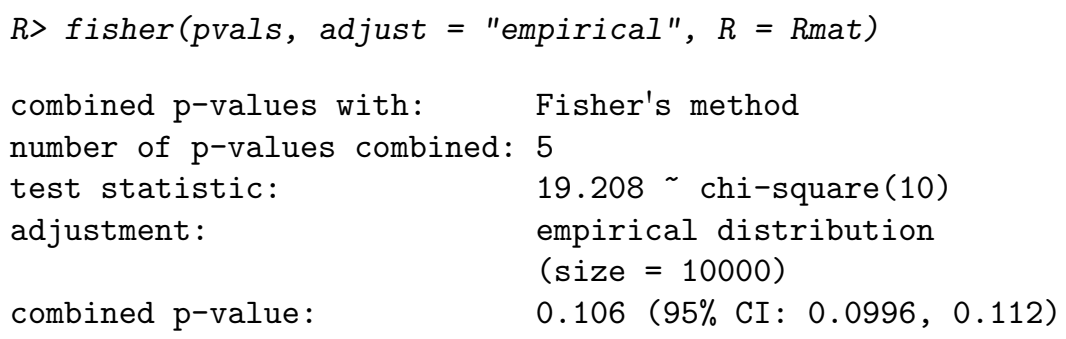


Under the assumption that the combined $p$-value represents an estimate of the 'true' combined $p$-value that would have been obtained under an infinite number of replications, the function also provides a confidence interval for the combined $p$-value based on the Clopper and Pearson [56] method (using stats: :binom.test() for the computations).

Although generating the null distribution using pseudo replicates is much faster than performing a 'proper' permutation test using the raw data, it is still computationally more demanding than the other adjustment techniques. The main factor that affects the computation time is the size of the empirical distribution generated. To reduce the computational burden, Liu et al. [18] proposed a stepwise algorithm that starts with a relatively small null distribution and only proceeds to generate a larger distribution when the combined $p$-value is small. The poolr package also provides the possibility to use such a stepwise algorithm via the size and threshold arguments of the base functions. In particular, for $j=1, \ldots$, length (size),

1. estimate the combined $p$-value based on size $[j]$,

2. if the combined $p$-value is $\geq$ than threshold $[j]$, stop (and report the combined $p$-value), otherwise go back to 1 .

By setting size to a vector with increasing values (e.g., size $=c(1000$, $100000,10000000)$ ) and threshold to decreasing values (e.g., threshold $=c(.10, .01,0))$, one can quickly obtain a fairly accurate estimate of the combined $p$-value if it is far from significant (e.g., $\geq .10$ ), but hone in on a more accurate estimate that is closer to 0 . Note that the last value of threshold should be 0 (and is forced to be inside of the functions), so that the algorithm is guaranteed to terminate (hence, one can also leave out the last value of threshold, so threshold $=c(.10, .01)$ would also work in the example above). For example:

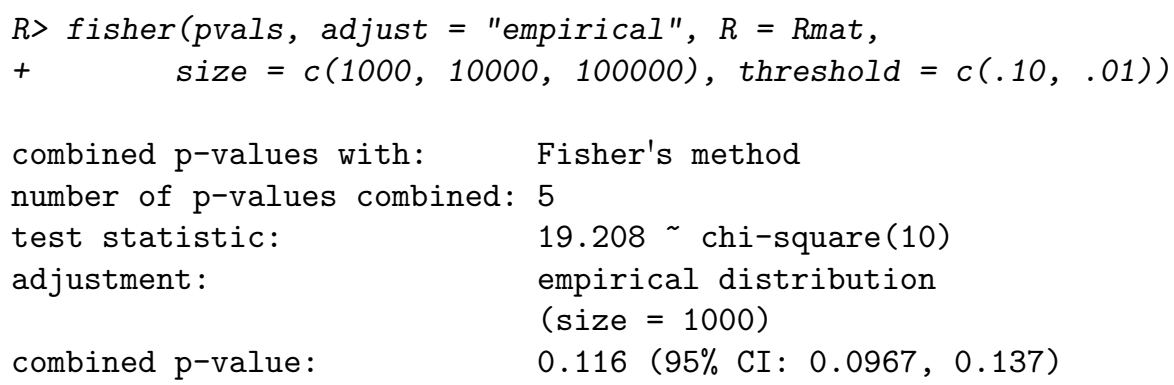


Since the combined $p$-value is just a bit larger than .10 (the first threshold) in this example, we have decreased the computational burden, since only 1000 values of the null distribution needed to be generated. While the difference may be hardly noticeable in the present case, when applying such methods repeatedly under circumstances where many combined $p$-values are likely to be insignificant (e.g., for a large number of genes in the GWAS setting), this approach can help to reduce the total computation time considerably. For example: ${ }^{6}$

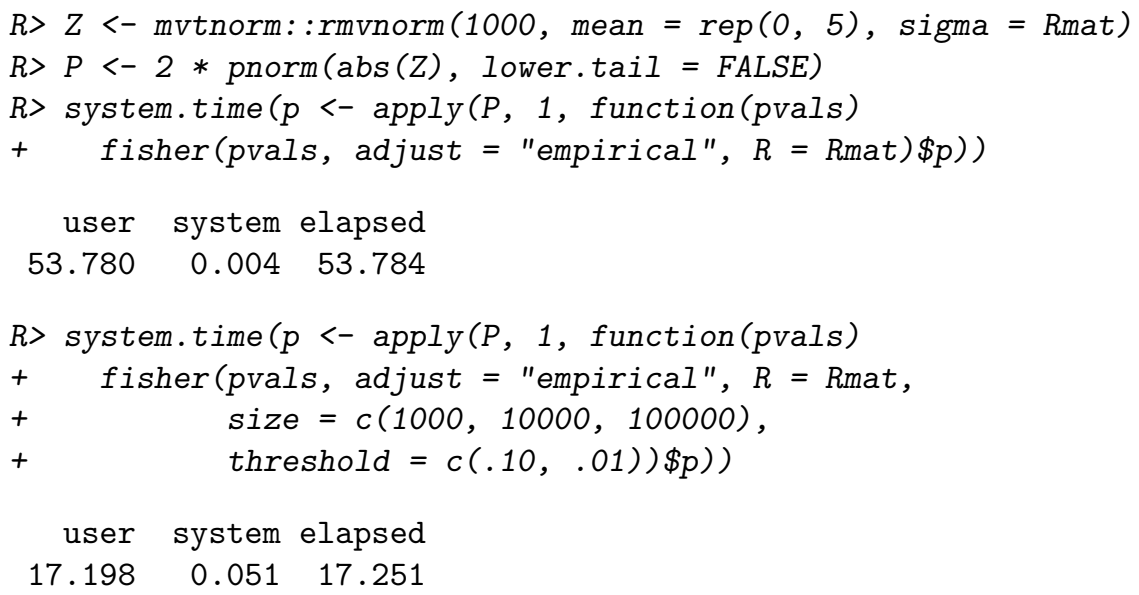

Generating the null distribution requires simulating a size $\times k$ matrix of pseudo test statistics. By default, this is done in a single step, so this entire matrix needs to be held in memory temporarily. When size and $k$ are very large, doing so can lead to memory allocation problems. In cases where this problem occurs, it can be avoided by dividing the process that generates the null distribution into multiple batches with smaller sizes, which can be done by setting the batchsize argument to some value smaller than size. Doing so reduces memory usage, but leads to an increase in the computation time:

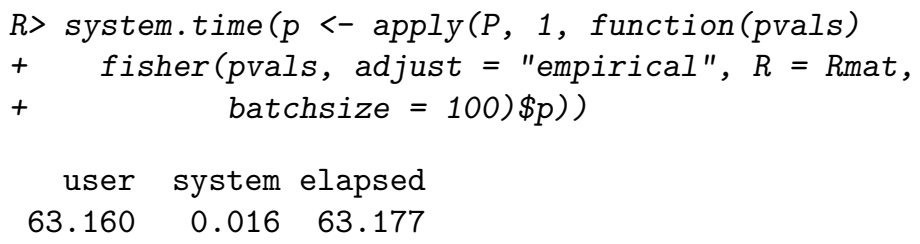

${ }^{6}$ All timings reported in this paper were obtained by running the code on an Intel Xeon E5-2630v4 processor. 
Note that size does not need to be an even multiple of batchsize (the size of the last batch will be adjusted appropriately such that the null distribution will always be of the requested size).

\section{Adjusting based on generalized methods}

As discussed earlier, the Fisher, Stouffer, and inverse chi-square methods can also be directly generalized to account for dependence. To make use of these methods, we set adjust $=$ "generalized" in the respective base functions. Moreover, $\mathrm{R}_{t}$ needs to be converted into a matrix that contains the covariances among the appropriate transformation of the test statistics before it can be passed to the functions via the $\mathrm{R}$ argument. In principle, this step requires repeatedly solving double integrals numerically, which is computationally very demanding. This problem can be easily sidestepped via a well-known memoization technique, namely the use of a lookup table that contains pre-computed values of these covariances for the various 'target' statistics. The dataset mvnlookup in the poolr package contains such a table:

\begin{tabular}{|c|c|c|c|c|c|c|c|c|}
\hline rhos & m2lp_1 & m2lp_2 & $z_{-} 1$ & $z_{-} \_2$ & chisq1_1 & chisq1_2 & $p_{-} 1$ & p_2 \\
\hline 1.000 & 4.0000 & 4.0000 & 1.000 & 1.0000 & 2.0000 & 2.0000 & 0.0833 & 8833 \\
\hline 0.999 & 3.9949 & 3.9908 & 0.999 & 0.9811 & 1.9971 & 1.9956 & 0.0832 & 0.0830 \\
\hline 0.998 & 3.9901 & 3.9823 & 0.998 & 0.9735 & 1.9944 & 1.9915 & 0.0831 & 0.0826 \\
\hline 0.997 & 3.9854 & 3.9738 & 0.997 & 0.9669 & 1.9917 & 1.9875 & 0.0831 & 0.0823 \\
\hline 0.996 & 3.9806 & 3.9653 & 0.996 & 0.9610 & 1.9891 & 1.9836 & 0.0830 & 0.0819 \\
\hline 0.995 & 3.9758 & 3.9568 & 0.995 & 0.9555 & 1.9864 & 1.9796 & 0.0829 & 0.0816 \\
\hline
\end{tabular}

Columns m2lp_1 and m2lp_2 correspond to (5.19) and (5.20), columns $z_{-} 1$ and $z_{-} 2$ to $(5.22)$ and (5.23), and columns chisq_1 and chisq_2 to (5.24) and (5.25), respectively. The last two columns are the covariances among oneand two-sided $p$-values, which we already made use of earlier (or rather, the correlations).

The values in the lookup table were computed using Gauss-Legendre quadrature with the help of the pracma package [57]. For this, we used a very fine grid with dimensions $1000 \times 1000$ on the interval $[-5,5]$ for both test statistics. Total construction time of the lookup table was about 45 hours using 18 cores in parallel on a workstation with two Intel Xeon E5-2630v4 processors. Values were cross-checked using adaptIntegrate() from the cubature package [58] and via simulations. 
Instead of using the table directly, mvnconv() conveniently allows the transformation of an entire $\mathrm{R}_{t}$ matrix into the required covariance matrix. The $\mathrm{R}_{t}$ matrix is passed to the function via the $\mathrm{R}$ argument, target should be set to either "m2lp", "z", or "chisq1" for functions fisher(), stouffer(), and invchisq(), respectively, and argument side to 1 or 2 depending on the sidedness of the tests (with 2 being the default). For example:

$R>\operatorname{mvnconv}($ Rmat, target $=" m 21 p ")$

$$
[, 1] \quad[, 2] \quad[, 3] \quad[, 4] \quad[, 5]
$$

$[1,] \quad \begin{array}{lllll}4.0000 & 1.9286 & 1.9286 & 1.9286 & 1.9286\end{array}$

$[2,] \quad \begin{array}{llllll}1.9286 & 4.0000 & 1.9286 & 1.9286 & 1.9286\end{array}$

$[3,] \quad \begin{array}{llllll}1.9286 & 1.9286 & 4.0000 & 1.9286 & 1.9286\end{array}$

$\left[\begin{array}{lllllll}4,] & 1.9286 & 1.9286 & 1.9286 & 4.0000 & 1.9286\end{array}\right.$

$\left[\begin{array}{llllll}5,] & 1.9286 & 1.9286 & 1.9286 & 1.9286 & 4.0000\end{array}\right.$

For convenience (and to reduce potential usage errors), when mvnconv() is used within a call to a base function with adjust = "generalized", the target argument is automatically set to the appropriate option. Hence, the following code will run the appropriate 'generalized' version of each test:

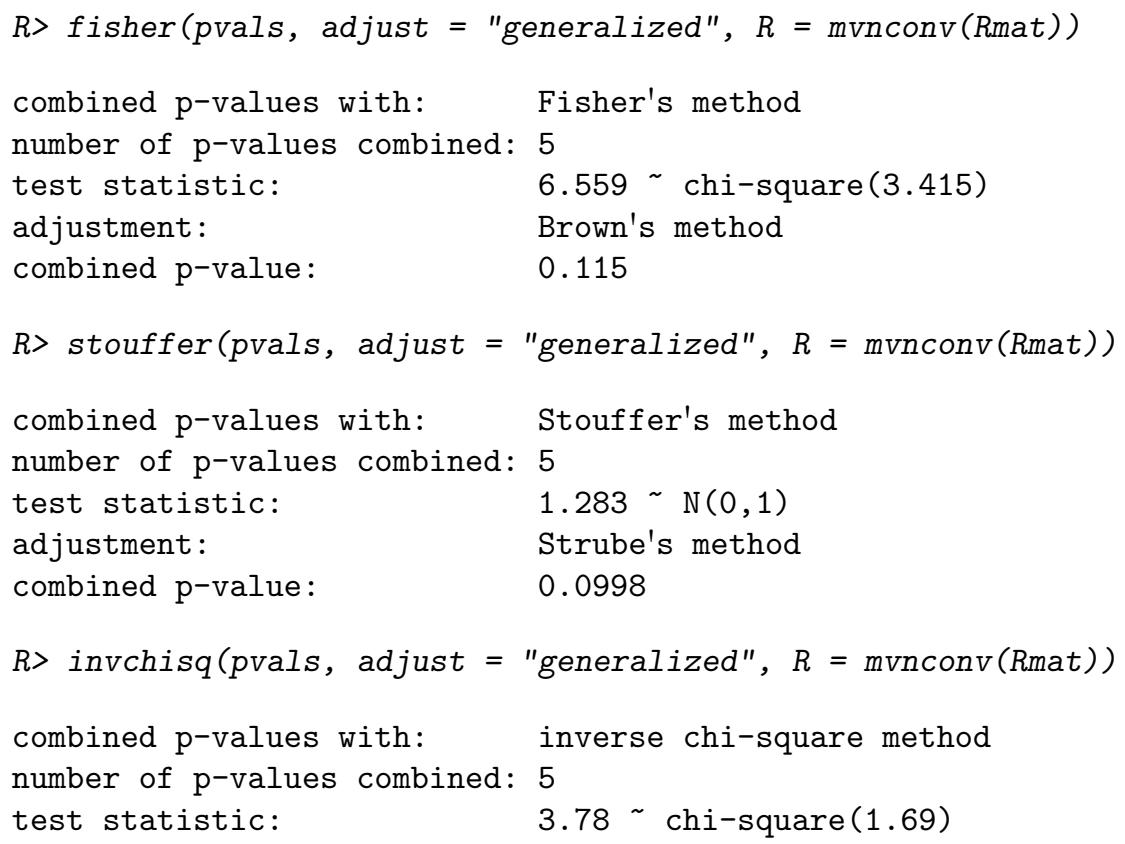


adjustment:

Satterthwaite approximation

combined p-value:

0.116

Although the results from Strube's method appear sensible here, we reiterate our caution about the use of this method for two-sided tests.

Note that all of the methods implemented in the poolr package are equally applicable to one-sided tests. For example, suppose that the test statistics simulated earlier were used to test one-sided hypotheses such that only positive values could lead to rejection. Then the one-sided p-values would be:

$R>$ pvals <- pnorm(ti, lower.tail = FALSE)

$R>$ round (pvals, digits $=5$ )

[1] $\begin{array}{lllllll}0.13228 & 0.15375 & 0.27697 & 0.02032 & 0.01842\end{array}$

Only two minor changes are now required to the code above. First, whenever mvnconv () is called, we need to set argument side $=1$, so that the appropriate column from the lookup table is used for converting the correlations to the covariances. For example:

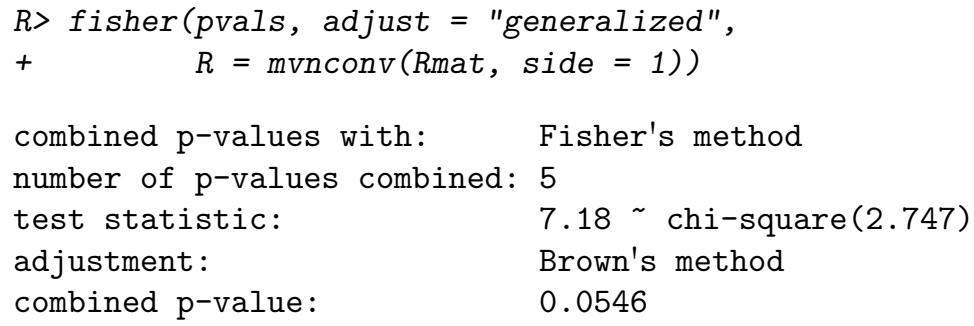

Moreover, when using adjust = "empirical" in any of the base functions, the side argument needs to be set in the same manner, so that the pseudo replicates of the test statistics are also appropriately converted into one-sided $p$-values. For example:

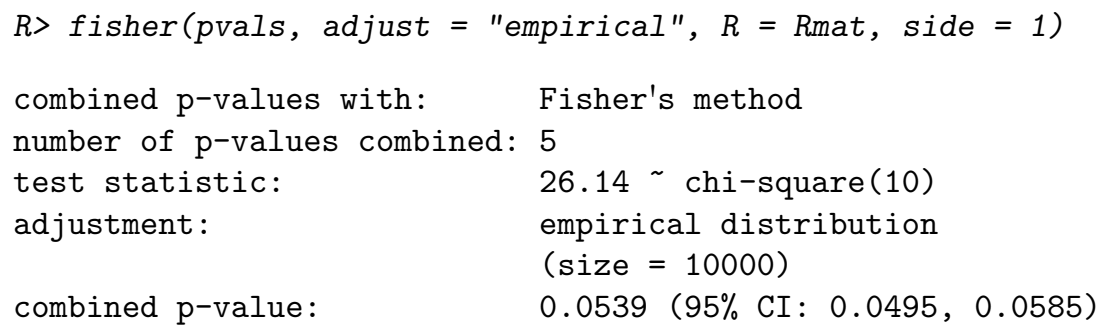




\subsection{Example}

The example above was based on simulated data. In this section, we illustrate the use of the poolr package using real data from a candidate-gene study by Van Assche et al. [59] who examined the association between 4947 SNPs and depressive symptoms, as measured with the Center of Epidemiologic Studies Depression Scale (CES-D Scale), in a sample of 982 adolescents. The $p$-values for the associations were obtained by fitting an additive model [46] to each SNP, with the CES-D as response variable. The SNPs were then grouped based on the gene to which they belonged (263 genes). Here, we focus on 23 SNPs that are part of the glutamate-related GRID2IP gene. The $p$-values of the first five SNPs in this gene were:

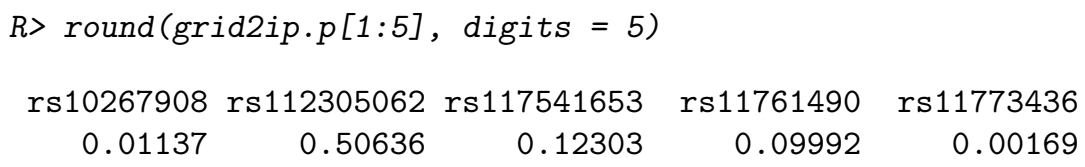

Object grid2ip.geno is a data frame with the genotypes for the SNPs, while grid2ip.pheno is a vector that contains the (log-transformed) CES-D values (note that these data frames only contain the data for the 886 adolescents with complete data on these 23 SNPs). Using the genetics package [60] for transforming the genotypes into the number of minor alleles in each SNP, the $23 p$-values can be recreated with:

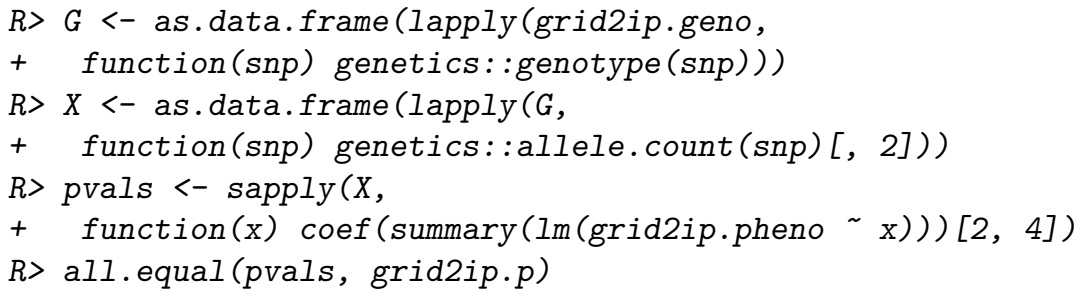

[1] TRUE

The corresponding $23 \times 23 \mathrm{LD}$ correlation matrix is stored in object grid2ip.ld. In Figure 5.1, we provide a heatmap of (the upper triangular part of) this correlation matrix. This correlation matrix can be recreated with:

$R>L D<-$ genetics: $: L D(G) \$ r$

$R>L D[$ lower.tri $(L D)]<-t(L D)$ [lower.tri $(L D)]$ 


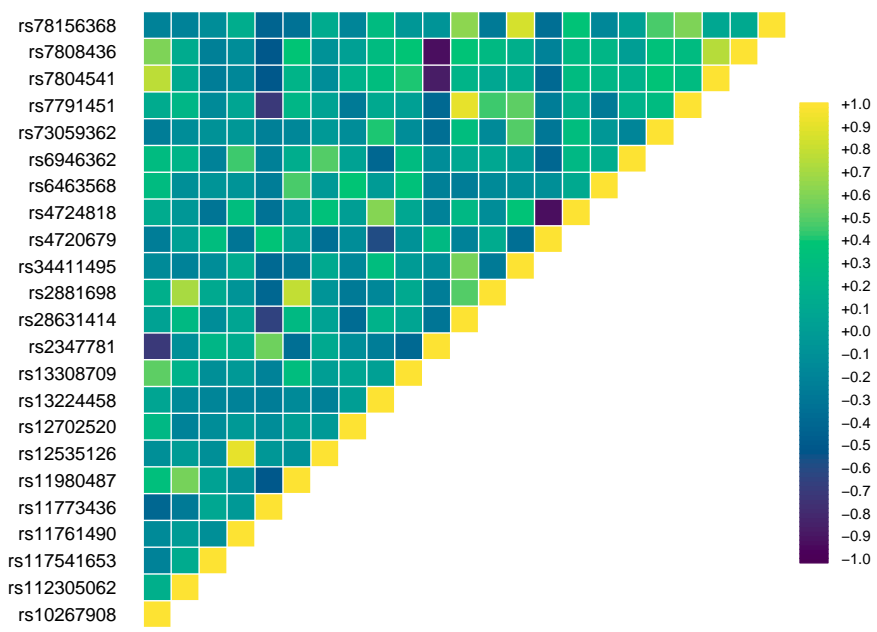

Figure 5.1: LD correlation matrix of 23 SNPs belonging to the GRID2IP gene.

$R>\operatorname{diag}(L D)<-1$

$R>$ all.equal (LD, grid2ip.ld)

\section{[1] TRUE}

Below we show how the combined $p$-value for this gene can be obtained by using Fisher's method along with all possible adjustments (only one effective number of tests adjustment is shown for brevity; the estimate of $m$ was 15 in this case). Note that the string passed to the adjust argument can also be abbreviated. Also, we use matrix LD (based on the interchanging elements) as an approximation to the correlations among the test statistics.

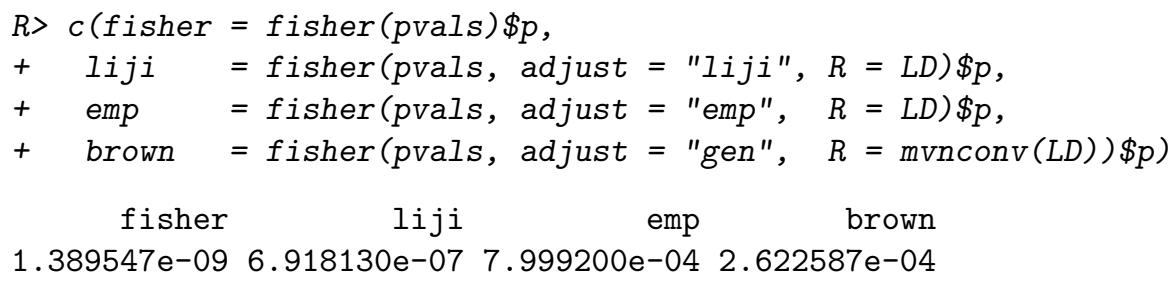

All of the combined $p$-values above agree that the gene is significantly associated with the phenotype of interest. For illustration purposes, we applied 

$p$-Values

all base methods and their adjustments to these data, yielding the results shown in Table 5.1 (results are given as $-\log _{10}\left(p_{c}\right)$-transformed values for easier comparison of very small values). Regardless of the base and adjustment method used, all combined $p$-values reached significance at $\alpha_{c}=.05$ (note: $\left.-\log _{10}(.05) \approx 1.301\right)$. However, we see considerable differences in the level of significance depending on the method used.

\begin{tabular}{lrrrrrrrr}
\hline method & base & nyholt & liji & gao & galwey & empirical & generalized & permutation \\
\hline fisher & 8.857 & 7.849 & 6.160 & 7.175 & 5.479 & 3.015 & 3.580 & 3.062 \\
stouffer & 8.781 & 7.761 & 6.051 & 7.079 & 5.362 & 3.134 & 3.948 & 3.176 \\
invchisq & 8.352 & 7.415 & 5.845 & 6.789 & 5.213 & 2.946 & 3.418 & 2.981 \\
binotest & 8.424 & 7.945 & 5.454 & 7.202 & 4.704 & 3.361 & & 3.381 \\
bonferroni & 1.411 & 1.472 & 1.597 & 1.517 & 1.659 & 1.518 & & 1.518 \\
tippett & 1.419 & 1.479 & 1.602 & 1.524 & 1.663 & 1.518 & & 1.518 \\
\hline
\end{tabular}

Table 5.1: Results ( $-\log _{10}\left(p_{c}\right)$-transformed values) when applying all base methods and adjustments to the 23 SNPs in the GRID2IP gene.

In the last column of the table, we also provide the combined $p$-values derived from conventional permutation tests using the raw data. For this, we tested the associations between the SNPs and the randomly reshuffled phenotype and then combined the resulting $p$-values with each of the base methods. After repeating this process $s=10^{6}$ times, we then computed the combined $p$-value for each base method with (5.18). In this example, the approach using pseudo replicates (also using size $=1 \mathrm{e}+06$ ) yielded almost identical results as the 'proper' permutation tests. However, while the latter took approximately 7 hours to complete, the former took less than 10 seconds per base method. ${ }^{7}$

\subsection{Comparison with other packages}

Several $\mathrm{R}$ packages and functions are already currently available to combine (dependent) $p$-values. In this section, we discuss their functionality and compare them to the poolr package based on the example data discussed in the previous section.

\footnotetext{
${ }^{7}$ In the permutation test, we used coef $(\operatorname{summary}(\operatorname{lm}(\ldots)))$ to extract $p$-values based on the permuted data in each iteration (similar to how we computed the observed $p$-values for the 23 SNPs earlier). The code underlying these functions carries out various pre- and post-processing steps that are not relevant for the present purposes. We can reduce the computation time considerably by using fastLmPure() from the RcppEigen package [61]. Doing so reduces the computation time to about 20 minutes.
} 
A first option is the p.adjust() function from the base package stats. This function provides a variety of methods for adjusting a set of $p$-values for multiple testing. As described earlier, this function can therefore also be used for combining $p$-values, although the Bonferroni correction and Holm's method are most relevant here, as they are the two methods available via this function that control the family-wise error rate under arbitrary assumptions. Using the example data, we can illustrate the equivalence of the Bonferroni and Holm methods and the implementation in the poolr package with:

$$
\begin{aligned}
& R>c(p . \text { adjust_bonferroni }=\min (p \text {.adjust }(p v a l s, \\
& +\quad \operatorname{method}=\text { "bonferroni")), } \\
& + \text { p.adjust_holm } \quad=\min (p . \operatorname{adjust}(p v a l s, \\
& +\quad \operatorname{method}=\text { "holm")), } \\
& + \text { poolr_bonferroni= bonferroni }(\text { pvals }) \$ p \text { ) } \\
& \begin{array}{rrr}
\text { p.adjust_bonferroni } & \text { p.adjust_holm } & \text { poolr_bonferroni }
\end{array}
\end{aligned}
$$

For researchers that want to use methods that were specifically developed for combining $p$-values, several packages are available (via CRAN and Bioconductor). The metap package [20] is the most comprehensive one, providing a wide variety of methods for combining independent $p$-values, including the ones part of the poolr package except for the Bonferroni method. Furthermore, the combine.test() function from the survcomp package [22] provides implementations of the Fisher and Stouffer methods and the logit-transformation method by George [21], whereas the aggregation package [23] provides functions for the Fisher, Tippett, and inverse chi-square methods (the latter being a special case of the method by [25]). Finally, the gap package [26] provides functions for Fisher's and Stouffer's methods. Below, we show how to apply these methods to the example data. ${ }^{8}$ The $-\log _{10}\left(p_{c}\right)$-transformed values provided in Table 5.2 demonstrate that results coincide across packages.

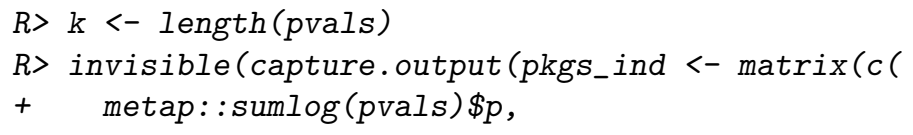

\footnotetext{
${ }^{8}$ Since gap::metap() always generates output via cat(), we use invisible(capture.output(...)) to suppress it. Also, for Stouffer's method, the function incorrectly divides the provided $p$-values by 2 , so we must double them first to obtain the correct result (it also incorrectly takes the absolute value of (5.2) before computing $p_{c}$, which does not affect results in the present case since $z$ is positive to begin with, but would have the effect of turning a set of really insignificant $p$-values into a significant combined $p$-value).
} 


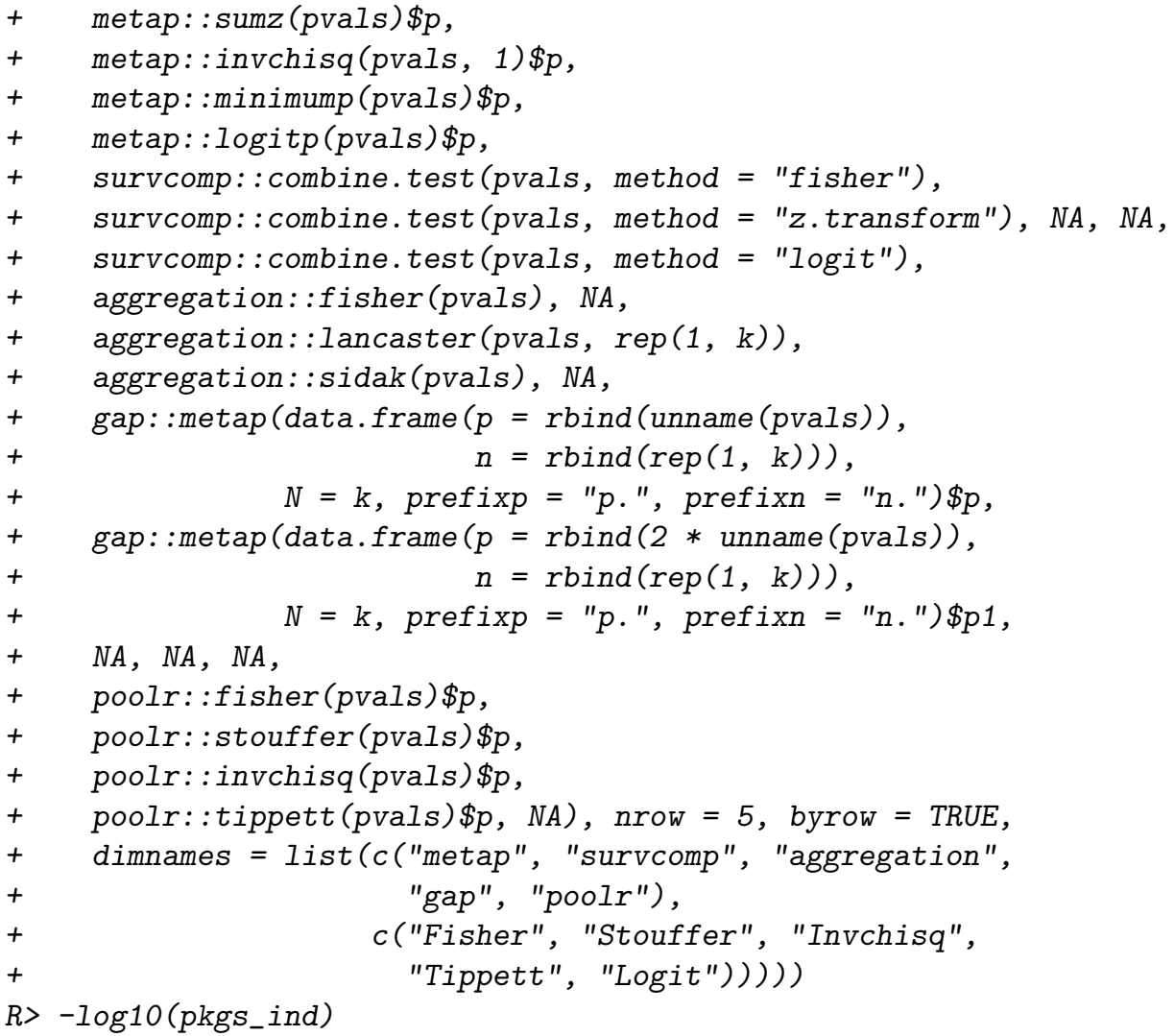

\begin{tabular}{lrrrrr}
\hline Package & Fisher & Stouffer & Invchisq & Tippett & Logit \\
\hline metap & 8.857127 & 8.781088 & 8.351928 & 1.419033 & 8.724881 \\
survcomp & 8.857127 & 8.781088 & & & 8.724881 \\
aggregation & 8.857127 & & 8.351928 & 1.419033 & \\
gap & 8.857127 & 8.781088 & & & \\
poolr & 8.857127 & 8.781088 & 8.351928 & 1.419033 & \\
\hline
\end{tabular}

Table 5.2: Results ( $-\log _{10}\left(p_{c}\right)$-transformed values) when applying various implementations of methods to the 23 SNPs in the GRID2IP gene that assume independence between the SNPs.

These results ignore the fact that the $23 p$-values to be combined are dependent. For combining dependent $p$-values, several other R packages are available. 
The CombinePValue package [28] provides an approach that is analogous to Brown's method, but (in addition to the actual $p$-values to be combined) requires the user to provide a matrix of $p$-values based on which the variancecovariance matrix of the $-2 \ln \left(p_{i}\right)$ will be estimated. In other words, instead of using analytic methods such as (5.19) and (5.20) to construct this variancecovariance matrix, it is estimated empirically. As described in the documentation, the matrix of $p$-values used for this step can be generated based on the raw data using resampling methods. Below, we construct such a matrix by repeatedly permuting the phenotype. Using the same variance-covariance matrix (after the $-2 \ln ()$ transformation) as input for $f$ isher () yields identical results. ${ }^{9}$

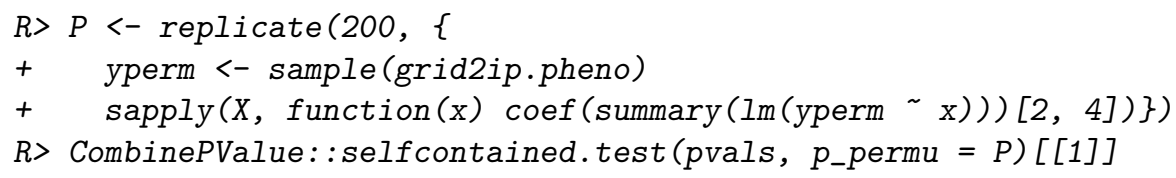

[1] $6.693278 \mathrm{e}-05$

$R>$ fisher(pvals, adjust $=$ "gen", $R=\operatorname{cov}(t(-2 * \log (P)))) \$ p$

\section{[1] $6.693278 \mathrm{e}-05$}

Ideally, one would want to increase the number of replicates, but doing so would be time intensive (e.g., for 10,000 replicates, the code above takes a bit over 4 minutes to complete, while fisher (pvals, adjust = "generalized", $\mathrm{R}=$ mvnconv (LD)) takes a fraction of a second). Moreover, if one generates the $p$-value matrix by resampling the raw data, one could just as well apply a proper permutation test directly as part of this process (instead of using this step only for generating the matrix that serves as input for the p_permu argument).

As another alternative, the EmpiricalBrownsMethod package [27] also implements a version of Brown's method and just like the CombinePValue package sidesteps the numerical integration but does so in a slightly different manner by required the user to input the raw data matrix that contains the interchanging elements. Each variable in this matrix is then transformed into percentiles using its empirical cumulative distribution function (as estimated via stats: : ecdf()) which in turn are $-2 \ln ()$-transformed. These

\footnotetext{
${ }^{9}$ Very minor discrepancies can occur in some cases because CombinePValue constrains $p$-values to fall in the interval $\left[10^{-6}, 1-10^{-6}\right]$, while no such restriction is applied in poolr.
} 
transformed values are then used to estimate the variance-covariance matrix of the $-2 \ln \left(p_{i}\right)$ values. Using the example data, one would therefore use this package as follows:

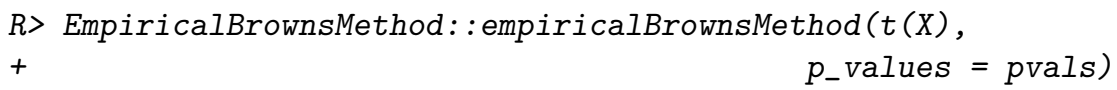

\section{[1] $3.288796 \mathrm{e}-09$}

If we manually apply the step that estimates the variance-covariance matrix, replace the diagonal values by 4 (which we know to be true and is also done by CombinePValue), and then use this matrix as input to fisher(), we obtain the same result:

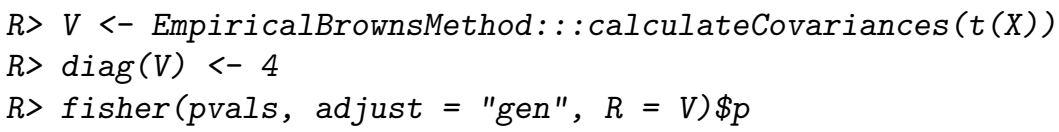

\section{[1] $3.288796 \mathrm{e}-09$}

However, this result seems 'too significant' compared to the combined $p$-value we obtained above when using the CombinePValue approach or Brown's method proper (recall that fisher (pvals, adjust = "gen", $\mathrm{R}=\operatorname{mvnconv}(\mathrm{LD})) \$ \mathrm{p}$ yields 0.000262$)$. There are two reasons for this discrepancy. First, in the present context, the variables in $\mathrm{X}$ (i.e., the number of minor alleles in each SNP) can only take on three unique values and so will the $-2 \ln ()$-transformed percentiles based on which the covariances are estimated. Hence, each covariance is estimated based on a $3 \times 3$ contingency table where the marginal distributions of the two variables are also often quite unbalanced. As a result, the covariances in $\mathrm{V}$ are much smaller than what they should be, at least when compared to $\operatorname{cov}(t(-2 * \log (P)))$ from above or when using the 'exact' covariances from mvnconv (LD, target $=$ "m2lp"). This leads to an undercorrection for dependence in Brown's method and hence to a combined $p$-value that is anticonservative. A way around this problem might be to simulate a data matrix from a multivariate normal distribution based on the same variance-covariance matrix as the original data and use this as input for the method: ${ }^{10}$

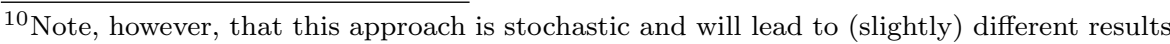
on repeated runs.
} 


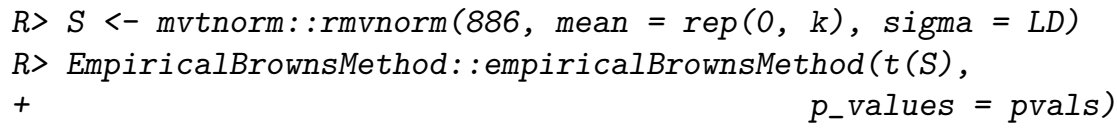

[1] $1.082403 e-05$

$R>V<-$ EmpiricalBrownsMethod: : calculateCovariances $(t(S))$

$R>\operatorname{diag}(V)<-4$

$R>$ fisher (pvals, adjust = "gen", $R=V$ ) $\$ p$

[1] $1.082403 e-05$

Although improved, this result still seems anticonservative. The reason for this is that the approach taken by empiricalBrownsMethod() implicitly assumes that the $p$-values to be combined are one-sided. We can demonstrate this by comparing the variance-covariance matrix obtained above with the one we obtain using mvnconv () when setting side $=1$ :

$R>\operatorname{round}($ unname $(V)$, digits $=4)[1: 4,1: 8]$

$\begin{array}{rrrrrrrrr} & {[, 1]} & {[, 2]} & {[, 3]} & {[, 4]} & {[, 5]} & {[, 6]} & {[, 7]} & {[, 8]} \\ {[1,]} & 4.0000 & 0.5769 & -0.6079 & -0.1057 & -1.2269 & 1.1555 & -0.0490 & 0.7288 \\ {[2,]} & 0.5769 & 4.0000 & 0.4935 & 0.0387 & -0.9727 & 2.2010 & 0.0343 & -0.6188 \\ {[3,]} & -0.6079 & 0.4935 & 4.0000 & -0.2153 & 0.3087 & 0.2160 & -0.2581 & -0.3923 \\ {[4,]} & -0.1057 & 0.0387 & -0.2153 & 4.0000 & -0.1508 & -0.2183 & 3.4120 & 0.0971\end{array}$

$R>\operatorname{mvnconv}(L D$, target $=" m 21 p "$, side $=1)[1: 4,1: 8]$

$[, 1] \quad[, 2] \quad[, 3] \quad[, 4] \quad[, 5] \quad[, 6] \quad[, 7] \quad[, 8]$

$\begin{array}{lllllllll}{[1,]} & 4.0000 & 0.6351 & -0.6006 & -0.4123 & -1.1635 & 1.1999 & -0.3036 & 0.8749\end{array}$

$\left[\begin{array}{llllllllll}{[2,]} & 0.6351 & 4.0000 & 0.4881 & -0.0326 & -0.8009 & 2.0748 & -0.0098 & -0.6245\end{array}\right.$

$\left[\begin{array}{lllllllll}{[3,]} & -0.6006 & 0.4881 & 4.0000 & -0.3099 & 0.3300 & 0.2184 & -0.3380 & -0.3752\end{array}\right.$

$\left[\begin{array}{lllllllll}{[4,]} & -0.4123 & -0.0326 & -0.3099 & 4.0000 & -0.0618 & -0.3005 & 3.5402 & -0.0876\end{array}\right.$

Clearly, the former is an approximation to the latter, while for two-sided $p$ values one should really use:

$R>\operatorname{mvnconv}(L D$, target $=" m 2 l p ")[1: 4,1: 8]$

$$
[, 1] \quad[, 2] \quad[, 3] \quad[, 4] \quad[, 5] \quad[, 6] \quad[, 7] \quad[, 8]
$$

$\left[\begin{array}{lllllllll}{[1,]} & 4.0000 & 0.1366 & 0.1440 & 0.0660 & 0.5922 & 0.4575 & 0.0352 & 0.2522\end{array}\right.$

$\left[\begin{array}{llllllllll}{[2,]} & 0.1366 & 4.0000 & 0.0821 & 0.0004 & 0.2642 & 1.2526 & 0.0000 & 0.1563\end{array}\right.$

$\left[\begin{array}{llllllllll}3,] & 0.1440 & 0.0821 & 4.0000 & 0.0367 & 0.0383 & 0.0170 & 0.0439 & 0.0544\end{array}\right.$

$\left[\begin{array}{lllllllllll}4,] & 0.0660 & 0.0004 & 0.0367 & 4.0000 & 0.0014 & 0.0345 & 3.2279 & 0.0028\end{array}\right.$ 
or more directly:

$R>$ fisher (pvals, adjust = "gen", $R=\operatorname{mvnconv}(L D)) \$ p$

[1] 0.0002622587

Hence, the EmpiricalBrownsMethod package, at least in its present form, should only be used for combining one-sided $p$-values and care must be taken when the interchanging variables have rather discrete distributions (e.g., as when using genotype data in GWAS).

In contrast to the previous two options, the TFisher package [29] provides an approximation to Brown's method that does not require access to the raw data. Although the manuscript does not explain how the covariances among the $-2 \log \left(p_{i}\right)$ values are approximated, the source code indicates that the package simply computes $4 \times \mathrm{R}^{2}$ (with the squaring done element-wise). Using the same approximation as input for the fisher() function yields the same combined $p$-value:

$R>1$ - TFisher: $p \cdot \operatorname{tpm}(\operatorname{sum}(-2 * \log ($ pvals $)), n=k, \operatorname{tau} 1=1, M=L D)$

[1] 0.0002822384

$R>$ fisher (pvals, adjust $=$ "gen", $\left.R=4 * L D^{\wedge} 2\right) \$ p$

\section{[1] 0.0002822384}

This result is quite close to the combined $p$-value obtained above with the 'exact' version of Brown's method. The explanation for this is provided by Figure 5.2, which shows that the simple approximation for the covariances used by TFisher is surprisingly accurate, deviating at most by -0.033 from the covariances computed based on (5.20). However, here we now have the opposite problem compared to EmpiricalBrownsMethod: Since it is not possible to change how the covariances are approximated, TFisher should only be used when combining two-sided $p$-values.

Finally, a rather different approach to combining $p$-values is implemented in the harmonicmeanp package [30]. In essence, the method uses the harmonic mean of the $p$-values, ${ }^{11}$

$$
\bar{p}=\frac{k}{\sum_{i=1}^{k} 1 / p_{i}},
$$

${ }^{11}$ We assume equal weights here; for the more general equation allowing different weights to be applied to the $p$-values, see Wilson [30]. 


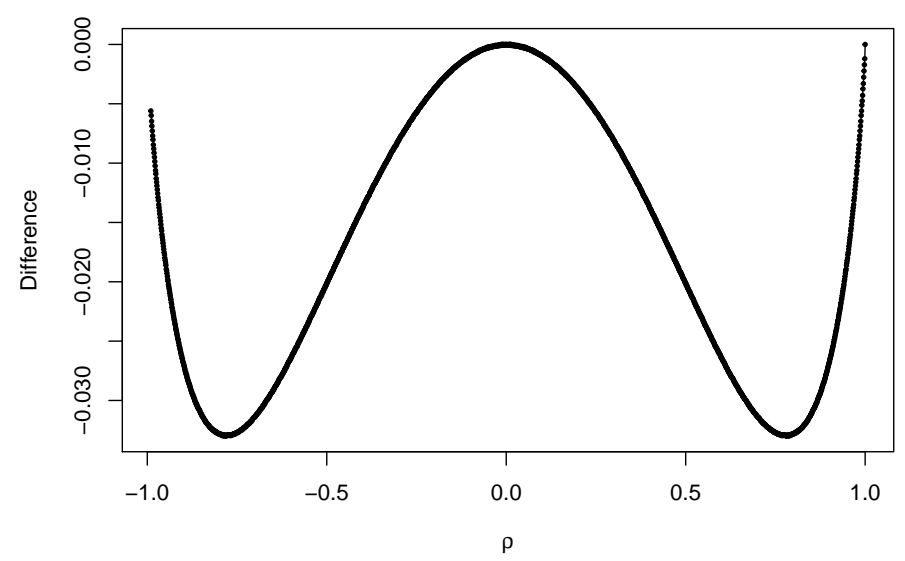

Figure 5.2: Difference between equation (5.20) and the covariances as approximated by the TFisher package as a function of $\rho$.

based on which an asymptotically exact combined $p$-value is computed using the Landau distribution (for details, see [30]). The method is robust to positive dependence among the $p$-values and does not require any further input other than the $p$-values to be combined:

$R>$ harmonicmeanp: :p.hmp (pvals, $L=k$ )

\section{p.hmp \\ 0.01302627}

Although significant, this result is more conservative than what we obtained with Brown's method. Further work is necessary to compare the methods implemented in poolr with the approach implemented in the harmonicmeanp package.

\subsection{Conclusion}

The poolr package provides a collection of methods for combining $p$-values that may be correlated due to non-independence among the tests from which 
they were derived. The package contains functions for a variety of 'base methods' for combining independent $p$-values along with several adjustments to these methods to account for dependence. Furthermore, the package provides logical extensions of various adjustments that have been described only incompletely in the literature and hence offers a more complete set of procedures.

For example, the base methods can be adjusted based on an estimate of the effective number of tests. However, only a subset of these adjustments have been described in the literature [e.g., 16]. In particular, the combination of this adjustment approach with the inverse chi-square method, the binomial method, and Stouffer's method is to our knowledge novel. Moreover, while the pseudo replication approach has been proposed in combination with the inverse chi-square method and the Bonferroni correction [17, 18], it can also be used in combination with any of the other methods. Finally, the use of the Satterthwaite approximation in combination with the inverse chi-square method is to our knowledge novel.

In this article, we illustrated the use of the poolr package with a dataset that was originally collected for a candidate-gene study. The same methods would be equally applicable to GWAS and other types of genetic studies (e.g., microarrays, DNA methylation analyses), studies using imaging techniques (e.g., functional magnetic resonance imaging), and essentially any situation where interest is not focused on the significance of individual tests (e.g., for SNPs, voxel), but of larger functional or structural units (e.g., genes, pathways, brain regions).

In applications of these types, researchers often want to compute a combined $p$-value for many such units. The total computation time can then become a major concern. Carrying out proper permutation tests for all units might in fact be computationally too demanding, at least for those without access to powerful hardware to parallelize the procedures. An obvious alternative approach - with a negligible computational burden - is to apply the Bonferroni method to the $p$-values within each unit, but this is often overly conservative. The methods implemented in the poolr package can be more powerful while still controlling the Type I error rate and at the same time are much less computationally demanding than permutation tests. Even the approach that mimics the latter can be carried out in seconds (as opposed to minutes or hours) through the use of pseudo replicates.

Moreover, an advantage of the methods implemented in poolr is that they do not require access to the raw data. The methods can be applied as long as the $p$-values are available and a matrix that reflects the correlations among 
the test statistics. How to obtain the latter however requires some thought. As we mentioned earlier, the correlations among the interchanging elements across analyses is often a good proxy. We can illustrate this point with a simple example. Suppose we test whether some continuous response variable of interest is associated with a number of dichotomous predictors. Let the correlation (i.e., the phi coefficient) between two of the predictors be:

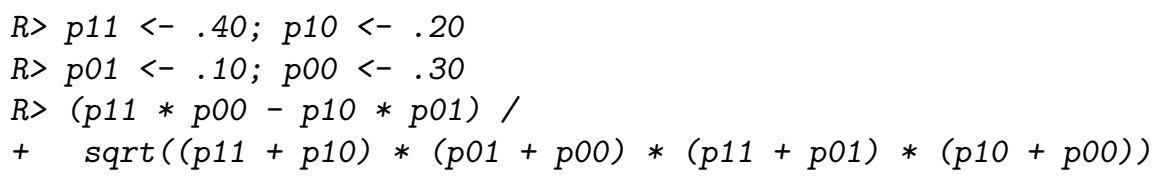

[1] 0.4082483

Now we repeatedly simulate data for $n=100$ individuals under this scenario and extract the test statistics. Their correlation is very close to that of the two predictors themselves:

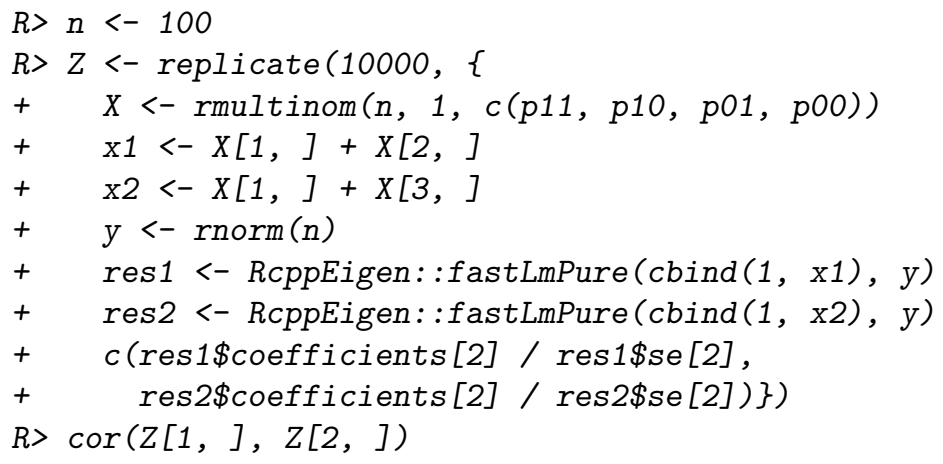

[1] 0.4112019

A further assumption underlying several of the methods is that the test statistics follow a multivariate normal distribution. In the example above, we know that the test statistics actually follow t-distributions with $\mathrm{df}=98$. However, from a practical point of view, this is hardly distinguishable from a normal distribution. To examine whether the assumption of joint multivariate normality is acceptable here, Figure 5.3 provides a filled contour plot of the joint distribution of the two test statistics (based on two-dimensional kernel density estimation obtained via MASS : $: \operatorname{kde} 2 \mathrm{~d}())$ and a QQ-plot of the Mahalanobis distances of the test statistics against the theoretical quantiles of a chi-square distribution with $\mathrm{df}=2$ (which, under the assumption of joint multivariate 
normality, is the asymptotic distribution of the Mahalanobis distances; [62]). Except for a few observations in the tail region, the assumption of multivariate normality appears to be an adequate approximation in this example.
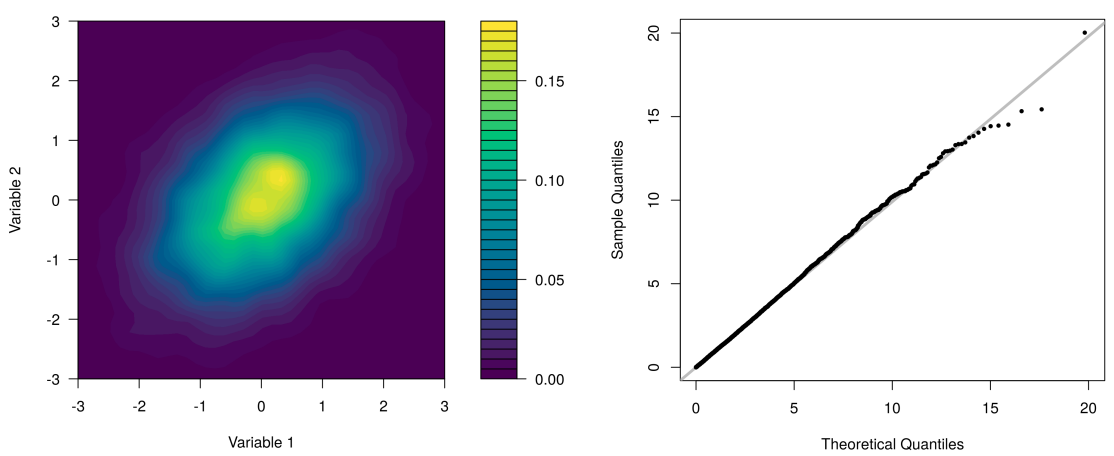

Figure 5.3: Filled contour plot showing the joint distribution of the test statistics and a QQ-plot of the Mahalanobis distances of the test statistics against the theoretical quantiles of a chi-square distribution with $\mathrm{df}=2$.

Simulations for other types of scenarios and models (e.g., with continuous but skewed predictors, with interchanging response variables as opposed to interchanging predictors, or for logistic regression models) suggest that the findings above hold with some generality as long as the sample size is sufficiently large. ${ }^{12}$ However, further work is required to delineate the specific circumstances under which (and how) the correlations among the interchanging elements can be mapped to the correlations among the test statistics.

For smaller sample sizes, the methods for converting the correlations among the test statistics into the covariances among the various target statistics (e.g., the $-2 \ln ()$-transformed $p$-values needed for Brown's method) may need to be adjusted [e.g., 53]. In future versions, we plan on expanding the package with additional lookup tables for other cases besides the multivariate normal one.

We may also consider expanding the package with additional base methods for combining $p$-values (and appropriate adjustments thereof to account for dependence). The choice of methods currently implemented was based on various

\footnotetext{
${ }^{12}$ For example, using logistic regression models with $\mathrm{x} 1$ and $\mathrm{x} 2$ as the response variables and $\mathrm{y}$ as the predictor in the simulation above yields essentially identical results.
} 
considerations, including the attention the various methods have received in the literature. The methods by Fisher and Stouffer are often discussed, especially as alternatives to 'proper' meta-analytic methods for combining evidence when only limited information about the studies/estimates to be combined is available [e.g., 63, 64, 65, 66]. Including the inverse chi-square method, these methods are also representative of the general class of methods that Loughin [67] describes as 'quantile combination methods', that is, they are all based on $\sum_{i=1}^{k} F^{-1}\left(p_{i}\right)$, where $F^{-1}(\cdot, 1)$ is the inverse of some cumulative distribution function (not necessarily for the chi-square distribution). In contrast, the Bonferroni and Tippett methods, and in a broad sense also the binomial test, belong to the class of 'order-statistic combination methods' that make use of the fact that the ranked $p$-values represent order statistics from a uniform distribution under the joint null hypothesis. The former two are particularly interesting due to their focus on the strongest 'signal' (which may be of interest in certain circumstances) while the latter has the intuitive appeal of being a method that looks for 'excess significance' in the set of $p$-values.

Our choices were also influenced by methods that have received heightened attention in the genetics literature, given that the development of the poolr package was motivated, at least in part, by our work in this area. Aside from Fisher's, the Bonferroni, and Tippett's methods, this in particular led to the inclusion of the inverse chi-square method [e.g., see 18, who discuss the use of this method in combination with the pseudo replication approach]. ${ }^{13}$ Although there are several other methods for combining $p$-values for general use proposed by Edgington [68] and George [21], they have not received as much attention as the aforementioned ones. Moreover, these two methods are currently only appropriate for combining independent $p$-values and are already available in the metap package.

Finally, we are fully aware of concerns about the overuse, overemphasis, or misinterpretation of $p$-values and potential dangers that may arise from drawing dichotomous conclusions based thereupon [e.g., see 69, and the corresponding supplementary materials]. Where possible, we therefore thoroughly support the call for increased emphasis on estimation in place of testing (with corresponding confidence intervals that reflect the uncertainty of the estimates) and the use of meta-analytic methods to combine such estimates [70]. However, in

\footnotetext{
${ }^{13}$ Also, popular tests in this area (such as the Cochran-Armitage trend test, the allelic association test, or tests based on a dominant, recessive, or heterozygote (dis)advantage model) all lead to one degree of freedom chi-square tests [47]. Then (5.3) is simply the sum of these chi-square statistics, which therefore suggests itself as a natural way of combining the information across multiple SNPs.
} 

p-Values

situations where we envision the use of the methods described in this paper (such as GWAS), there may not be any consistent directionality of the effects such that their combination using standard meta-analytic methods would be meaningful (e.g., the minor alleles at a locus may either be protective or harmful and this may differ across SNPs within a gene). Hence, methods based on the combination of $p$-values may still have their place and we believe that the poolr package provides a useful collection of methods for this purpose. 


\section{References}

[1] R. A. Fisher. Statistical Methods for Researchers (4th. ed.) Edinburgh, UK: Oliver and Boyd, 1932.

[2] B. J. Becker. "Combining significance levels". In: The handbook of research synthesis. Ed. by H. Cooper and L. V. Hedges. New York: Russell Sage Foundation, 1994, pp. 215-230.

[3] R. D. Cousins. Annotated Bibliography of Some Papers on Combining Significances or P-Values. Tech. rep. University of California, 2008.

[4] R. Rosenthal. "Combining Results of Independent Studies". In: Psychological Bulletin 85.1 (1978), pp. 185-193. DOI: 10.1037/0033-2909.85. 1.185.

[5] S. A. Stouffer et al. The American Soldier: Adjustment During Army Life (Studies in Social Psychology in World War II. Vol. 1. Princeton: Princeton University Press, 1949.

[6] B. Wilkinson. "A Statistical Consideration in Psychological Research". In: Psychological Bulletin 48.2 (1951), pp. 156-158. DoI: https://doi. org/10.1037/h0059111.

[7] B. Lehne, C. M. Lewis, and T. Schlitt. "From SNPs to Genes: disease Association at the Gene Level". In: PLoS One 6.6 (2011), e20133. DOI: https://doi.org/10.1371/journal.pone.0020133.

[8] M. Slatkin. "Linkage Disequilibrium-Understanding the Evolutionary Past and Mapping the Medical Future". In: Nature Reviews Genetics 9.6 (2008), pp. 477-485. DOI: https://doi.org/10.1038/nrg2361.

[9] G. Alves and Y. K. Yu. "Accuracy Evaluation of the Unified P-Value from Combining Correlated P-Values". In: PLoS One 9.3 (2014), e91225. DOI: https://doi.org/10.1371/journal.pone. 0103662.

[10] M. B. Brown. "400: A Method for Combining Non-Independent, OneSided Tests of Significance". In: Biometrics (1975), pp. 987-992. DOI: https://doi.org/10.2307/2529826.

[11] M. J. Strube. "Combining and Comparing Significance Levels from Nonindependent Hypothesis Tests". In: Psychological Bulletin 97.2 (1985), pp. 334-341. DOI: https://doi.org/10.1037/0033-2909.97.2.334.

[12] J. M. Cheverud. "A Simple Correction for Multiple Comparisons in Interval Mapping Genome Scans". In: Heredity 87.1 (2001), pp. 52-58. DOI: https://doi.org/10.1046/j.1365-2540.2001.00901.x. 
[13] D. R. Nyholt. "A simple correction for multiple testing for single-nucleotide polymorphisms in linkage disequilibrium with each other". In: Am J Hum Genet 74.4 (2004), pp. 765-9. DOI: $10.1086 / 383251$.

[14] J. Li and L. Ji. "Adjusting multiple testing in multilocus analyses using the eigenvalues of a correlation matrix". In: Heredity (Edinb) 95.3 (2005), pp. 221-7. DOI: $10.1038 / \mathrm{sj}$.hdy.6800717.

[15] X. Gao, J. Starmer, and E. R. Martin. "A multiple testing correction method for genetic association studies using correlated single nucleotide polymorphisms". In: Genet Epidemiol 32.4 (2008), pp. 361-9. DOI: 10 . 1002/gepi. 20310.

[16] N. W. Galwey. "A new measure of the effective number of tests, a practical tool for comparing families of non-independent significance tests". In: Genet Epidemiol 33.7 (2009), pp. 559-68. DOI: 10.1002/gepi. 20408.

[17] D. Y. Lin. "An Efficient Monte Carlo Approach to assessing Statistical Significance in Genomic Studies". In: Bioinformatics 21.6 (2005), pp. 781-787. DOI: https : // doi . org/10 . 1093/bioinformatics / bti053.

[18] J. Z. Liu et al. "A Versatile Gene-Based Test for Genome-Wide Association Studies". In: The American Journal of Human Genetics 87.1 (2010), pp. 139-145. DOI: https://doi.org/10.1016/j.ajhg.2010.06.009.

[19] V. Moskvina et al. "Evaluation of an Approximation Method for Assessment of Overall Significance of Multiple Dependent Tests in a Genomewide Association Study". In: Genetic Epidemiology 35.8 (2011), pp. 861-866. DOI: https://doi.org/10.1002/gepi. 20636.

[20] Michael Dewey. metap: Meta-Analysis of Significance Values. R package version 1.2. 2019.

[21] E. O. George. "Combining Independent One-Sided and Two-Sided Statistical Tests: Some Theory and Applications". PhD Dissertation. University of Rochester, 1977.

[22] M S Schroeder et al. "survcomp: An R/Bioconductor Package for Performance Assessment and Comparison of Survival Models". In: Bioinformatics 27.22 (2011), pp. 3206-3208. DOI: $10.1093 /$ bioinformatics / btr511.

[23] Lynn Yi and Lior Pachter. aggregation: p-Value Aggregation Methods. $\mathrm{R}$ package version 1.0.1. 2018. 
[24] L. H. C. Tippett. The Methods of Statistics. London: Williams \& Norgate, 1931.

[25] H. O. Lancaster. "The Combination of Probabilities: An Application of Orthonormal Functions". In: Australian Journal of Statistics 3.1 (1961), pp. 20-33. DOI: $10.1111 / \mathrm{j} .1467-842 \mathrm{X} .1961$.tb00058.x.

[26] J H Zhao. "gap: Genetic Analysis Package". In: Journal of Statistical Software 23.8 (2007), pp. 1-18. DOI: $10.18637 /$ jss.v023.i08.

[27] William Poole. EmpiricalBrownsMethod: Uses Brown's Method to Combine P-Values from Dependent Tests. R package version 1.14.0. 2019.

[28] H. Dai, J. S. Leeder, and Y. Cui. "A Modified Generalized Fisher Method for Combining Probabilities from Dependent Tests". In: Frontiers in Genetics 5 (2014), p. 32. DOI: 10.3389/fgene.2014.00032.

[29] Hong Zhang et al. "TFisher Tests: Optimal and Adaptive Thresholding for Combining p-Values". In: arXiv preprint arXiv:1801.04309 (2018).

[30] Daniel J Wilson. "The Harmonic Mean P-Value for Combining Dependent Tests". In: Proceedings of the National Academy of Sciences 116.4 (2019), pp. 1195-1200. DOI: 10.1101/171751.

[31] J. J. Goeman and A. Solari. "Multiple Hypothesis Testing in Genomics". In: Statistics in Medicine 33.11 (2014), pp. 1946-1978. DOI: https : // doi.org/10.1002/sim.6082.

[32] R. J. Simes. "An Improved Bonferroni Procedure for Multiple Tests of Significance". In: Biometrika 73.3 (1986), pp. 751-754. DoI: https : // doi.org/10.1093/biomet/73.3.751.

[33] S. Holm. "A Simple Sequentially Rejective Multiple Test Procedure". In: Scandinavian Journal of Statistics 6 (1979), pp. 65-70.

[34] Y. Hochberg. "A Sharper Bonferroni Procedure for Multiple Tests of Significance". In: Biometrika 75.4 (1988), pp. 800-802. DoI: https : // doi.org/10.1093/biomet/75.4.800.

[35] G. Hommel. "A Stagewise Rejective Multiple Test Procedure Based on a Modified Bonferroni Test". In: Biometrika 75.2 (1988), pp. 383-386. DOI: https://doi.org/10.1093/biomet/75.2.383.

[36] Y. Benjamini and Y. Hochberg. "Controlling the False Discovery Rate: A Practical and Powerful Approach to Multiple Testing". In: Journal of the Royal Statistical Society, Series B (Methodological) (1995), pp. 289-300. DOI: https://doi.org/10.1111/j.2517-6161.1995.tb02031.x. 
[37] Yoav Benjamini and Daniel Yekutieli. "The control of the false discovery rate in multiple testing under dependency". In: Annals of statistics (2001), pp. 1165-1188.

[38] O. J. Dunn. "Estimation of the Means of Dependent Variables". In: Annals of Mathematical Statistics 29.4 (1958), pp. 1095-1111. DOI: https: //doi.org/10.1214/aoms/1177706443.

[39] Z. Šidák. "Rectangular Confidence Regions for the Means of Multivariate Normal Distributions". In: Journal of the American Statistical Associations 62.318 (1957), pp. 626-633. DOI: https : //doi .org/10.2307/ 2283989 .

[40] A. W. van der Vaart. Asymptotic Statistics. New York: Cambridge University Press, 1998.

[41] W. K. Härdle and L. Simar. Applied Multivariate Statistical Analysis. 4th. New York: Springer, 2015.

[42] M. H. DeGroot and M. J. Schervish. Probability and Statistics. 4th. Boston: Pearson, 2011.

[43] P. Good. Permutation Tests: A Practical Guide to Resampling Methods for Testing Hypotheses. Springer Science \& Business Media, 2013.

[44] P. H. Westfall and S. S. Young. Resampling-Based Multiple Testing: Examples and Methods For P-Value Adjustment. New York: Wiley, 1993.

[45] R. C. Johnson et al. "Accounting for Multiple Comparisons in a GenomeWide Association Study (GWAS)". In: BMC Genomics 11.1 (2010), p. 724. DOI: https://doi.org/10.1186/1471-2164-11-724.

[46] N. M. Laird and C. Lange. The Fundamentals of Modern Statistical Genetics. New York: Springer, 2010.

[47] A. Ziegler and I. R. König. A Statistical Approach to Genetic Epidemiology: Concepts and Applications, with an E-Learning Platform. 2nd. Weinheim, Germany: Wiley, 2010.

[48] A. Birnbaum. "Combining Independent Tests of Significance". In: Journal of the American Statistical Association 49.267 (1954), pp. 559-547. DOI: 10. $2307 / 2281130$.

[49] M. D. Ernst. "Permutation Methods: A Basis for Exact Inference". In: Statistical Science 19.4 (2004), pp. 676-685. DOI: 10.1214/088342304000000396. 
[50] K. N. Conneely and M. Boehnke. "So many correlated tests, so little time! Rapid adjustment of P values for multiple correlated tests". In: Am J Hum Genet 81.6 (2007), pp. 1158-68. DOI: 10.1086/522036.

[51] B. Phipson and G. K. Smyth. "Permutation P-Values Should Never Be Zero: Calculating Exact P-Values When Permutations Are Randomly Drawn". In: Statistical Applications in Genetics and Molecular Biology 9.1 (2010). DOI: 10.2202/1544-6115. 1585.

[52] James J Yang et al. "An efficient genome-wide association test for multivariate phenotypes based on the Fisher combination function". In: $B M C$ bioinformatics 17.1 (2016), p. 19.

[53] J. T. Kost and M. P. McDermott. "Combining Dependent P-Values". In: Statistics and Probability Letters 60 (2002), pp. 183-190. DOI: 10.1016/ S0167-7152(02)00310-3.

[54] P. Billingsley. Probability and Measure. New York: Wiley, 1995.

[55] S. I. Resnick. A Probability Path. New York: Springer, 2014. DOI: 10. 1007/978-0-8176-8409-9.

[56] Charles J Clopper and Egon S Pearson. "The Use of Confidence or Fiducial Limits Illustrated in the Case of the Binomial". In: Biometrika 26.4 (1934), pp. 404-413. DOI: 10.1093/biomet/26.4.404.

[57] Hans W. Borchers. pracma: Practical Numerical Math Functions. R package version 2.2.9. 2019.

[58] Balasubramanian Narasimhan et al. cubature: Adaptive Multivariate Integration over Hypercubes. R package version 2.0.4. 2019.

[59] Evelien Van Assche et al. "Gene-Based Interaction Analysis Shows GABA Ergic Genes Interacting with Parenting in Adolescent Depressive Symptoms". In: Journal of Child Psychology and Psychiatry 58.12 (2017), pp. 1301-1309. DOI: 10.1111/jcpp.12766.

[60] Gregory Warnes et al. genetics: Population Genetics. R package version 1.3.8.1.2. 2019 .

[61] D. Bates and D. Eddelbuettel. "Fast and Elegant Numerical Linear Algebra Using the RcppEigen Package". In: Journal of Statistical Software 1.5 (2013), pp. 1-24. DOI: 10.18637/jss.v052.i05.

[62] M. J. R. Healy. "Multivariate Normal Plotting". In: Journal of the Royal Statistical Society, Series C 17.2 (1968), pp. 157-161. DOI: 10.2307 / 2985678. 
[63] N. M. Laird and F. Mosteller. "Some Statistical Methods for Combining Experimental Results". In: International Journal of Technology Assessment in Health Care 6.1 (1990), pp. 5-30.

[64] J. E. Schmid, G. G. Koch, and L. M. LaVange. "An Overview of Statistical Issues and Methods of Meta-Analysis". In: Journal of Biopharmaceutical Statistics 1.1 (1991), pp. 103-120.

[65] National Research Council. Combining Information: Statistical Issues and Opportunities. Washington, DC: National Academic Press, 1992.

[66] W. W. Piegorsch and A. John Bailer. "Combining Information". In: Wiley Interdisciplinary Reviews: Computational Statistics 1.3 (2009), pp. 354360 .

[67] T. M. Loughin. "A Systematic Comparison of Methods for Combining P-Values From Independent Tests". In: Computational Statistics 83 Data Analysis 47.3 (2004), pp. 467-485.

[68] Eugene S Edgington. "An Additive Method for Combining Probability Values from Independent Experiments". In: The Journal of Psychology 80.2 (1972), pp. 351-363. DOI: 10.1080/00223980.1972.9924813.

[69] Ronald L Wasserstein, Nicole A Lazar, et al. "The ASA's Statement on P-Values: Context, Process, and Purpose". In: The American Statistician 70.2 (2016), pp. 129-133. DOI: 10.1080/00031305.2016.1154108.

[70] G. Cumming. Understanding the New Statistics: Effect Sizes, Confidence Intervals, and Meta-Analysis. New York: Routledge, 2012. 
6

Discussion 
The combination of information is a powerful tool to reach more efficient and generalizable results and it intuitively fits with the cumulative nature of science. Information can be combined in various ways employing different types of statistical procedures. For example, researchers can synthesize information from primary studies and quantify the heterogeneity among them by means of a meta-analysis. Alternatively, information from multiple sources can be combined based on limited data by using methods for combining $p$-values. Although meta-analytic methods and methods for combining $p$-values are wellknown and have been well-studied, there is still more to examine to improve their use. In this thesis, some of these issues are examined based on simulation studies. Here, the findings of these studies are discussed and linked to each other and the literature. Furthermore, caveats and possible future directions are going to be considered.

\subsection{Model Selection in Meta-Regression}

The precision of a meta-analysis can be improved by associating the effect size of interest with the characteristics of the primary studies. This approach can also explain the between-study heterogeneity in the effect size that arises from differences in study characteristics $[1,2]$. Specifically, this improvement works by employing conventional regression-type methods that use covariates for the study characteristics (such as spatial [3] or treatment [4] differences) to associate them with the effect sizes; an analysis known as meta-regression where the covariates are known as "moderators".

Similar to regular regression analyses, the goal is to identify a model that provides a close approximation to the true data generation process underlying the data [1]. Therefore, model selection should be carefully handled to identify such a model. As a conventional strategy in traditional regression analyses, selection via univariate testing or full model testing can be employed in metaregression. However, univariate testing requires multiple testing adjustments to control the Type I error rate which is neither common nor well-studied in meta-regression. Alternatively, one can use information-theoretic techniques such as selection via information criteria, multimodel inference, and relative variable importance.

In Chapter 2, we examined the performance of model selection techniques in meta-regression by means of a simulation study. The results showed that model selection via testing performs poorly in general. Univariate testing had a very low probability of identifying the true model in almost all conditions 
examined in the simulation. In meta-regression, moderators are often correlated with each other and such correlations negatively impact the performance of univariate testing methods. That is, irrelevant moderators can be found to be significant due to their correlation with relevant moderators. Full model testing, on the other hand, proved to be more promising in identifying the true model. It could even outperform information-theoretic methods under certain circumstances, such as when the sample size is small. However, it should be noted that all of the techniques had little chances of identifying the true model under such circumstances and the more complex methods considered usually outperformed full model testing as the sample size or the strength of the association between moderators and effect sizes increased.

The results of Chapter 2 also brought new insights to a debate in the use of complex model selection techniques in meta-regression. The debate arises in estimating the likelihoods of the candidate models which can be made by maximum likelihood (ML) or restricted maximum likelihood (REML) procedures. Pinheiro and Bates [5] and Verbeke and Molenberghs [6] have argued that REML estimation is not appropriate to compare models with different fixed effects. In contrast, Gurka [7] presented a simulation study whose results showed that this is not entirely true as long as AIC or AICc criteria are employed for model selection. Chapter 2 showed that REML estimation can perform adequately in identifying the true model from candidate models that differ in their fixed effects. In addition, the reduced REML procedure provided a substantial benefit to the model selection techniques for identifying the true model when it is an "empty" model (i.e., when none of the moderators in the candidate set are truly related to the effect sizes).

A noteworthy finding of Chapter $\mathbf{2}$ is that multimodel inference particularly has a high probability of identifying the true model when it is an empty model. This adequate performance when the size of the coefficient of the moderators is small (or absent) could alternatively be explained by the approach that assigns 0 weights to the candidate models where the parameter is absent. Anderson [8] argued that this approach provides a shrinkage effect; it obtains an estimate that is always smaller than the estimate that would be derived by using only the models that include the parameter of interest, which in turn improves the model selection when the true model is empty. In this regard, Chapter 2 supports a previous study by Lukacs, Burnham, and Anderson [9] which reported that multimodel inference outperforms stepwise algorithms (a means of selection via testing) in identifying the true empty model. However, our study also emphasizes the importance of using the reduced REML procedure in such cases as other complex model selection techniques also performed well 
in identifying the true empty model with this procedure. For example, selection via information criteria only worked well when the likelihoods were derived by the reduced REML procedure in such cases.

When the true model includes significant moderators, however, multimodel inference was outperformed by some of the other information-theoretic techniques. In these cases, selection via information criteria and relative variable importance (with a cutoff value of 0.5) performed reasonably well regardless of the information criterion. Their advantage was more observable as the size of the coefficient of the moderators and the sample size increased. In addition, the reduced REML procedure also had a slight advantage over the other likelihood procedures, especially with selection via information criteria, when the true model is not empty. Here, it should be emphasized that the cutoff values used for selecting variables based on their relative variable importance are completely arbitrary, and the technique itself is not a principled one. Anderson [8] argued that such a ranking variable technique should be acknowledged more as an exploratory tool rather than a confirmation analysis. Yet, Chapter 2 showed that relative variable importance can be an effective exploratory tool (for some examples in ecology, see $[10,11,12,13]$ ).

\subsection{Combining Information from Dependent Sources}

Synthesizing information from correlated data appears in this dissertation in two contexts which will be discussed separately in the following sections. The common issue in both contexts in the presence of correlated data is that most conventional statistical methods assume independence and not addressing the correlation is likely to give rise to efficiency problems.

\subsubsection{Meta-Analysis in Ecology}

Ecology studies commonly examine different species, and meta-analyses in ecology need to synthesize information from these species. Conventional metaanalytic methods assume independence among the effect sizes. However, due to the shared evolutionary history among the species, known as a phylogeny, the effect sizes are correlated, violating the independence assumption. Furthermore, ecology studies commonly report multiple effect sizes which is another characteristic that should be handled carefully in meta-analyses. 
Phylogenies have been incorporated into statistical models with phylogenetic comparative methods, which in turn have been shown to decrease the Type I error rate $[14,15,16,17,18,19]$. Phylogeny has also been integrated into meta-analysis with methods proposed by Adams [20] and Lajeunesse [21] that employ fixed- and random-effects models, respectively. In a later study, Chamberlain et al. [22] showed that these models can prevent Type I errors by applying them on data sets from previous studies that ignore the phylogeny; however, neither model can handle multiple effect sizes reported by primary studies. In this regard, one can employ a multilevel mixed-effects model that can address both the phylogeny and multiple effect sizes. Yet, the performance of this more complex model has not so far been compared to conventional metaanalytic models. In Chapter 3, we conducted a simulation study to examine the effects of incorporating the phylogeny in a meta-analysis using a multilevel mixed-effects model.

The results of Chapter $\mathbf{3}$ showed that the point estimation of the overall mean does not change with regards to the complexity of the meta-analytic model. However, the uncertainty in this estimation can be captured most accurately only by the multilevel meta-analytic model that incorporates the phylogeny. Therefore, ignoring the phylogeny inflates the risk of a Type I error considerably. In this regard, Chapter $\mathbf{3}$ extends the argument made by Chamberlain et al. [22] to the multilevel modeling framework. Nevertheless, one potential danger arises when the number of effect sizes is small where all of the meta-analytic models overestimate the overall mean. Only the multilevel mixed-effects model that includes the phylogeny could capture the uncertainty in the overall mean estimation sufficiently well to avoid an inflated Type I error rate.

The significance of the phylogenetic signal can be tested through descriptive statistics such as $\lambda$ and $K$ in phylogenetic comparative studies [14, 23]. Analogously, a statistic of similar means can be derived from the most complex model using the two variance components it includes. The strength of the phylogenetic signal among the species can be investigated by using $\frac{\sigma_{p}^{2}}{\sigma_{n}^{2}+\sigma_{p}^{2}}$. Furthermore, the strength of the phylogenetic signal can be tested using likelihood ratio tests by employing the most complex model and a model that excludes $\sigma_{p}^{2}$. Lynch [24] also proposed a way to estimate the standard error of this ratio which could be useful to perform a Wald-type test to examine the significance of the phylogenetic signal. However, the distribution of this ratio is not known explicitly; thus, such a test may not guarantee a nominal rejection level until further examination. 
As aforementioned, the most complex model under evaluation in Chapter 3 includes two random-effects components for non-phylogenetic and phylogenetic variances. The results showed that the model could estimate the true values of these variance components with little or no bias. However, the bias in their estimation may increase substantially when there is a weak phylogeny. In a general sense, when the average correlation in the phylogeny (excluding the diagonal values) is lower than 0.2 , researchers should take the estimations of these variance components with caution. The results suggested that the most complex model can estimate the total variance from the non-phylogenetic and phylogenetic components correctly; however, both of them are estimated roughly as half of this total variance, which introduces bias to both estimations when their true values are not equal. Therefore, roughly equal estimations for these two variance components under a weak phylogeny may be an indicator to the researchers that the estimations are biased.

An important result of this study could warn researchers about a risk in common practice. The multilevel meta-analytic model is a complex statistical model; therefore, it may require sufficient data to adequately estimate the parameters. Some meta-analyses in ecology have tried to reduce the complexity of the meta-analytic model by removing the non-phylogenetic variance component (see [25, 26] for examples). However, the results of Chapter 3 showed that removing the non-phylogenetic variance component widens the confidence interval for the overall mean too much and the model becomes too conservative. Therefore, such a simplified model should be avoided. Furthermore, we did not witness severe convergence issues when fitting the most complex model. Especially when the true model that underlies the data does include non-zero variance components, the most complex model does not experience convergence issues, and can be fitted to the data in a reasonable amount of time. On the other hand, when the true model is a simpler one than the most complex model assumes, the model experiences little, but not severe, convergence issues (less than $5 \%$ of the time).

\subsubsection{Combining $p$-Values in Genetics}

Genome-wide association studies (GWAS) examine the contribution of mutations on individual bases on the human genome known as single-nucleotide polymorphisms (SNPs) on a disease of interest. In contemporary GWAS, the number of SNPs examined can easily be more than a million and conventional multiple testing procedures are too severe under such large numbers of simultaneous tests. As an alternative, gene-based testing that combines evidence 
from SNPs in gene regions is a common approach in GWAS. Gene-based testing can employ methods for combining $p$-values; however, an essential part of this task is to address the nonrandom associations among the SNPs known as linkage disequilibrium (LD). In Chapter 4, we reviewed the most common methods for combining $p$-values and adjustment techniques to incorporate the LD information into this process.

The results of the simulation in Chapter $\mathbf{4}$ showed that the unadjusted methods for combining $p$-values cannot keep the rejection rates at the nominal level. As expected, the Bonferroni and Tippett methods behaved overly conservative; therefore, they both could lead to a severe loss of power. On the other hand, the binomial test, Fisher's method, and Stouffer's method lead to too liberal results, inflating the Type I error rates. Therefore, all the unadjusted techniques should be avoided when combining dependent $p$-values.

Although some adjustment techniques may provide nominal rejection rates, not all seem promising in this regard. For example, estimating the effective number of tests does not guarantee nominal rejection rates. Most of these adjustments fail to reject the true null hypothesis at nominal levels. Conneely and Boehnke [27] argued that the effective number of tests is a single parameter that cannot sufficiently explain the dependence structure. Yet, the Li \& Ji adjustment can provide a nominal rejection rate with the Bonferroni and Tippett methods and performs adequately with the binomial test. Furthermore, the Galwey adjustment also provides rejection rates close to the nominal level (although slightly liberal) with the Bonferroni and Tippett methods. These two adjustments have also been shown to be promising by Wen and $\mathrm{Lu}$ [28]; therefore, they could be examined in additional studies to study their behavior in further contexts.

Permutation-based techniques are acknowledged as the gold standard to combine dependent $p$-values. However, the heavy computational burden of such techniques makes them difficult to apply in GWAS. One possible way to circumvent this problem is to mimic the permutation-based techniques using empirical methods. That is, it is possible to generate the null distribution of the combined $p$-value under the null hypothesis, assuming that the test statistics follow a multivariate normal distribution with a covariance matrix that is identical to the LD matrix. The performance of such empirical approaches have been shown to be similar to that of proper permutation tests in studies that use the Bonferroni and Fisher's methods [27, 29, 30, 31]. Chapter 4 shows that such empirical methods may behave conservatively with the Bonferroni, Tippett, and binomial test methods. However, Fisher's and Stouffer's methods 
provide rejection rates at the nominal level using the empirical methods. In addition, Chapter $\mathbf{5}$ shows that these empirical methods can mimic proper permutation tests when combined with all methods for combining $p$-values. Therefore, empirical methods can replace the permutation-based techniques as the former decreases the computer burden substantially. However, Fisher's and Stouffer's methods might be preferred as they are more likely to provide a nominal rejection rate than the remaining methods.

The last technique to adjust the methods for combining $p$-values is to compute the test statistic under dependence assuming multivariate normality. One wellknown example was shown by Brown [32] that derives the combined Fisher's test statistic under dependence for one-sided tests. An extension of Brown's method to two-sided tests has been proposed by Yang et al. [33], although it has not been widely applied yet, and its performance has not been examined to address the LD information. Chapter $\mathbf{4}$ and Chapter $\mathbf{5}$ present the combined test statistics for Fisher's, Stouffer's, and the inverse chi-square (only in the latter chapter) methods for both one- and two-sided tests using a Satterthwaite approximation. The simulation in Chapter 4 shows that the default Brown's method for one-sided tests cannot control the Type I error rate at the nominal level when the tests are two-sided. However, the correct extension of Brown's method for two-sided tests provides rejection rates closest to the nominal level. Furthermore, the simulation in this chapter shows that the extended Brown's method is robust to the LD characteristics in genes such as the number of SNPs in a gene or the strength of the correlations among the SNPs. In addition, this adjustment also provides the highest power among the methods that can control the rejection rate at the nominal level. Therefore, researchers should carefully use the correct form of Brown's method when combining $p$-values derived from two-sided tests.

Although the extension of Brown's method to two-sided tests performs adequately, the Stouffer method under dependence does not provide this advantage. Strube [34] showed a general extension to the Stouffer method, and in Chapter 4, we generalized this extension to two-sided tests. The combined test statistic for the Stouffer method follows a normal distribution with the assumptions mentioned above; therefore, it should be an exact method. However, when the tests are two-sided, after transforming the two-sided $p$-values to the normally distributed test statistics, the combined test statistic does not follow a normal distribution anymore. Thus, using the Satterthwaite approximation cannot guarantee a nominal rejection rate. The simulation in Chapter 4 shows that Strube's method leads to slightly liberal rejection rates when the $p$-values are derived from two-sided tests. 


\subsection{Tips for Analysts}

This dissertation includes studies that evaluate methods for model selection in meta-regression, for meta-analyzing studies with complex dependency structures, and for combining dependent $p$-values. Although the studies here are focused on ecology and genetics, the statistical methods under examination can be utilized in other contexts. Here, I summarize some potentially useful strategies that researchers can follow in their studies.

First, Chapter 2 examined model selection techniques in meta-regression. Meta-regression uses conventional regression-type methods, and moderators (i.e., covariates) to be included in the model are commonly selected with selection via testing methods. However, our study showed that it is best to avoid such techniques as information-theoretic techniques outperform them. Our results showed that multimodel selection has a very high chance to identify the true model when it is an empty model. Therefore, it might be a good strategy to check if multimodel testing suggests an empty model. However, it should also be noted that multimodel testing may miss the true model when it is not an empty model. Thus, it is best to employ other techniques, especially selection via information criteria and relative variable importance with a cutoff value of 0.5 , to see if they also indicate a non-empty model. From a likelihood perspective, the (reduced) REML procedure seems to have adequate performance, especially when the true model is empty. It also performs adequately when there are significant moderators; however, it is best to check if there is consistency between the model indicated by different likelihood procedures in such cases. Likewise, the information criterion employed does not seem to lead to substantial differences in such cases; thus, it might also be useful to check the consistency when different information criteria are used.

Next, Chapter 3 compared the performance of a complex multilevel model that addresses phylogeny in ecological meta-analyses to more conventional meta-analytic models. Our results showed that the model provides coverage rates closest to the nominal level. Therefore, it is suggested to employ this model in a case where the researcher suspects that there is correlation among the effect sizes of interest derived from multiple interchanging elements (such as species). One common hesitation in the use of this model arises from its complexity which may lead to convergence issues and demand a large sample size. Some researchers have used a simplified model that exclude some of the variance components (non-phylogenetic variance component in phylogeny studies) to avoid such issues. However, our results showed that such a simplified model is prone to behave in an overly conservative way and may increase 
the Type II error rate. Furthermore, we found that the complex model does not have convergence issues especially when the data have the structure assumed by this model. Therefore, it can be suggested to fit the complex multilevel model to the data when there is a suspicion of such a correlated structure. If it experiences convergence problems, then meta-analysts may suspect that a correlation structure (such as phylogeny) does not play a role in the data, and they may try to use less complex models. One disadvantage of the complex model arises when the phylogenetic signal is weak. In such cases, the model usually estimates the non-phylogenetic and phylogenetic variance components as half of the sum of these two variance components. Thus, researchers may want to check for this phenomenon, which could be an indication of biased estimates when the phylogenetic signal is weak.

Finally, Chapter 4 and Chapter $\mathbf{5}$ examined methods for combining dependent $p$-values in the context of genetics, and in GWAS more specifically. Conventional methods for combining $p$-values such as Fisher's method do not address dependency among the $p$-values. None of these methods can control the Type I error rate at the nominal level and should be avoided. Although it is possible to address the dependency with effective number of tests techniques, they also failed in controlling the Type I error rate in general. Only the Li \& Ji technique (and sometimes the Galwey technique) could be appropriate when combined with the Bonferroni (and Tippett) method. On the other hand, the methods that derive the empirical distribution under the null hypothesis seem to provide adequate results with the Fisher and Stouffer methods. Furthermore, our results showed that these empirical techniques can mimic proper permutation-based techniques which is a clear advantage as the former can derive the results much faster than the latter does. Finally, the generalization of Fisher's method under dependence, Brown's method, is the most robust technique. However, it should be highly emphasized that the default Brown's method can be applied only to one-sided tests; therefore, one should employ its extension to two-sided tests when $p$-values are derived from two-sided tests. All the methods examined in these two chapters are available in an open-source $\mathrm{R}$ software package called poolr which is freely available to use.

\subsection{Limitations and possible future studies}

Finally, I would like to point out some missing aspects of the studies presented in this thesis and raise some ideas for future possibilities. There are two main limitations and some smaller issues that would be interesting to address 
in the future. The first main issue applies to the meta-analytic studies in Chapter 2 and Chapter 3. In the former chapter, we generated standardized mean differences as the effect size, whereas in the latter chapter, we generated effect sizes directly from normal distributions. Given that standardized mean differences have an approximately normal distribution, it is possible to argue that both of these studies examine the same type of effect size. However, other types of effect sizes, such as odds ratios, are also common in meta-analyses. Other types of effect sizes may follow a normal distribution only approximately; therefore, the results of these two chapters may not be completely generalizable to studies that use different effect size types. Extending these studies using other types of effect sizes could be a beneficial addition to the literature to address this issue.

Second, we did not discuss or include missing data in these chapters. Missing values occur frequently and require careful attention to be addressed. Listwise deletion is commonly applied to deal with missing data in analyses; however, the loss of information that comes with this approach may severely harm the quality of the analysis. For example, Ellington et al. [35] presented the consequences that may arise with listwise deletion and suggest to use multiple imputation techniques in meta-analysis. However, missing value imputation techniques have not been studied extensively, especially in meta-regression; therefore, a future study on this issue could also be a good addition to the literature.

Missing data may also arise in GWAS in a different form. As shown in Chapter 4 and Chapter 5, adjusting the methods for combining $p$-values to control the dependency among the tests assumes that the correlation among the interchanging elements (i.e., LD) is known. However, this information may be missing from the analysts. In the absence of the correlation information, there are two possible ways to follow. First, one can derive the LD values from well-known genome databases such as ENSEMBL. A useful tool for this is an R package, LDlinkR [36], with which the users can connect to the ENSEMBL database and derive the LD values. As an alternative, one can try to estimate the correlation directly from the $p$-values themselves by assuming a compound symmetric correlation matrix. For example, Makambi [37] proposed a way to estimate the correlation among the $p$-values using the method-of-moments. Other methods, such as maximum likelihood estimation, may be more efficient in this regard; however, this is yet to be examined in a future study.

Apart from these points, there are a few more issues that deserve attention. First, univariate testing for model selection in meta-regression is found to be an 
insufficient technique in Chapter 2. However, it is known that this technique has a multiple testing issue, and we did not focus on this aspect. Therefore, the performance of this technique when its reports are adjusted by multiple testing corrections has yet to be examined. Next, combining dependent $p$ values in GWAS also has several points to be addressed. For example, the use of LD derived from the allelic data (a binary variable) may be an issue for such analyses since the $p$-values are derived from genotype data typically coded as $0 / 1 / 2$ (for the number of minor alleles). Therefore, deriving the correlations among the SNPs using the genotype data, known as compound linkage disequilibrium (CLD), might be a more appropriate approach. Furthermore, LD is derived from the sample at hand and hence its accuracy depends on the sample size. Since well-known databases provide LD data from much larger samples, deriving the LD information from the databases rather than computing them from the sample at hand may be more efficient. Finally, another idea to combine the dependent $p$-values can be made by using a multivariate chi-square distribution. However, these issues should be examined in further studies. 


\section{References}

[1] S. G. Thompson and J. P. T. Higgins. "How should meta-regression analyses be undertaken and interpreted?" In: Statistics in Medicine 21.11 (2002), pp. 1559-1573.

[2] Julia Koricheva and Jessica Gurevitch. "Uses and misuses of meta-analysis in plant ecology". In: Journal of Ecology 102.4 (2014), pp. 828-844.

[3] Graham A Colditz et al. "Efficacy of BCG vaccine in the prevention of tuberculosis: meta-analysis of the published literature". In: Jama 271.9 (1994), pp. 698-702.

[4] Malcolm R Law, Nicholas J Wald, and SG Thompson. "By how much and how quickly does reduction in serum cholesterol concentration lower risk of ischaemic heart disease?" In: Bmj 308.6925 (1994), pp. 367-372.

[5] J. C. Pinheiro and D. M. Bates. Mixed-effects models in $S$ and S-PLUS. New York: Springer, 2000.

[6] G. Verbeke and G. Molenberghs. Linear mixed models for longitudinal data. New York: Springer, 2000.

[7] M. J. Gurka. "Selecting the best linear mixed model under REML". In: American Statistician 60.1 (2006), pp. 19-26.

[8] D. R. Anderson. Model based inference in the life sciences: A primer on evidence. 2nd. New York: Springer, 2007.

[9] P. M. Lukacs, K. P. Burnham, and D. R. Anderson. "Model selection bias and Freedman's paradox". In: Annals of the Institute of Statistical Mathematics 62.1 (2010), pp. 117-125.

[10] Masashi Soga and Shinsuke Koike. "Relative importance of quantity, quality and isolation of patches for butterfly diversity in fragmented urban forests". In: Ecological Research 27.2 (2012), pp. 265-271.

[11] M Soga and S Koike. "Mapping the potential extinction debt of butterflies in a modern city: implications for conservation priorities in urban landscapes". In: Animal Conservation 16.1 (2013), pp. 1-11.

[12] Masashi Soga et al. "Landscape versus local factors shaping butterfly communities in fragmented landscapes: Does host plant diversity matter?" In: Journal of Insect Conservation 19.4 (2015), pp. 781-790. 
[13] Johanna M Toivonen et al. "Environmental drivers of vascular and nonvascular epiphyte abundance in tropical pre-montane cloud forests in Northern Peru". In: Journal of Vegetation Science 28.6 (2017), pp. 1198 1208 .

[14] Simon P Blomberg, Theodore Garland Jr, and Anthony R Ives. "Testing for phylogenetic signal in comparative data: behavioral traits are more labile". In: Evolution 57.4 (2003), pp. 717-745.

[15] Rob P Freckleton, Paul H Harvey, and Mark Pagel. "Phylogenetic analysis and comparative data: a test and review of evidence". In: The American Naturalist 160.6 (2002), pp. 712-726.

[16] Paul H Harvey and Andy Purvis. "Comparative methods for explaining adaptations". In: Nature 351.6328 (1991), p. 619.

[17] Paul H Harvey, Mark D Pagel, et al. The comparative method in evolutionary biology. Vol. 239. Oxford university press Oxford, 1991.

[18] Anthony R Ives, Peter E Midford, and Theodore Garland Jr. "Withinspecies variation and measurement error in phylogenetic comparative methods". In: Systematic Biology 56.2 (2007), pp. 252-270.

[19] Rafael Molina-Venegas and Miguel Á Rodrıguez. "Revisiting phylogenetic signal; strong or negligible impacts of polytomies and branch length information?" In: BMC evolutionary biology 17.1 (2017), p. 53.

[20] Dean C. Adams. "Phylogenetic meta-analysis". In: Evolution 62.3 (Mar. 2008), pp. 567-572. DOI: 10.1111/j.1558-5646.2007.00314.x.

[21] Marc J Lajeunesse. "Meta-analysis and the comparative phylogenetic method". In: The American Naturalist 174.3 (2009), pp. 369-381.

[22] S. A. Chamberlain et al. "Does phylogeny matter? Assessing the impact of phylogenetic information in ecological meta-analysis". In: Ecology Letters 15.6 (2012), pp. 627-36.

[23] Mark Pagel. "Inferring the historical patterns of biological evolution". In: Nature 401.6756 (1999), p. 877.

[24] Michael Lynch. "Methods for the analysis of comparative data in evolutionary biology". In: Evolution 45.5 (1991), pp. 1065-1080.

[25] László Zsolt Garamszegi, Gábor Markó, and Gábor Herczeg. "A meta-analysis of correlated behaviours with implications for behavioural syndromes: mean effect size, publication bias, phylogenetic effects and the role of mediator variables". In: Evolutionary Ecology 26.5 (2012), pp. 1213-1235. 
[26] Fhionna R Moore, David M Shuker, and Liam Dougherty. "Stress and sexual signaling: a systematic review and meta-analysis". In: Behavioral ecology 27.2 (2015), pp. 363-371.

[27] K. N. Conneely and M. Boehnke. "So many correlated tests, so little time! Rapid adjustment of P values for multiple correlated tests". In: Am J Hum Genet 81.6 (2007), pp. 1158-68. DOI: 10.1086/522036.

[28] S. H. Wen and Z. S. Lu. "Factors Affecting the Effective Number of Tests in Genetic Association Studies: A Comparative Study of Three PCABased Methods". In: Journal of Human Genetics 56.6 (2011), pp. 428435. DOI: https://doi.org/10.1038/jhg.2011.34.

[29] J. Z. Liu et al. "A Versatile Gene-Based Test for Genome-Wide Association Studies". In: The American Journal of Human Genetics 87.1 (2010), pp. 139-145. DOI: https://doi.org/10.1016/j.ajhg.2010.06.009.

[30] V. Moskvina et al. "Evaluation of an Approximation Method for Assessment of Overall Significance of Multiple Dependent Tests in a Genomewide Association Study". In: Genetic Epidemiology 35.8 (2011), pp. 861-866. DOI: https://doi.org/10.1002/gepi. 20636.

[31] D. Y. Lin. "An Efficient Monte Carlo Approach to assessing Statistical Significance in Genomic Studies". In: Bioinformatics 21.6 (2005), pp. 781-787. DOI: https : // doi . org/10 . 1093/bioinformatics / bti053.

[32] M. B. Brown. "400: A Method for Combining Non-Independent, OneSided Tests of Significance". In: Biometrics (1975), pp. 987-992. DOI: https://doi.org/10.2307/2529826.

[33] James J Yang et al. "An efficient genome-wide association test for multivariate phenotypes based on the Fisher combination function". In: $B M C$ bioinformatics 17.1 (2016), p. 19.

[34] M. J. Strube. "Combining and Comparing Significance Levels from Nonindependent Hypothesis Tests". In: Psychological Bulletin 97.2 (1985), pp. 334-341. DOI: https://doi .org/10.1037/0033-2909.97.2.334.

[35] E Hance Ellington et al. "Using multiple imputation to estimate missing data in meta-regression". In: Methods in Ecology and Evolution 6.2 (2015), pp. 153-163.

[36] Timothy A. Myers, Stephen J. Chanock, and Mitchell J. Machiela. "LDlinkR: An R Package for Rapidly Calculating Linkage Disequilibrium Statistics in Diverse Populations". In: Frontiers in Genetics 11.157 (2020). DOI: 10.3389/fgene.2020.00157. 
[37] Kepher Makambi. "Weighted inverse chi-square method for correlated significance tests". In: Journal of Applied Statistics 30.2 (2003), pp. 225234. 


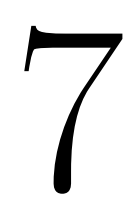

General Summary 
In this thesis, some aspects of statistical methods for combining information are examined. There are two main contexts in general: meta-analysis and combining $p$-values. A common issue present in most of the dissertation is the combination of non-independent data. Furthermore, the thesis include an examination of model selection techniques in meta-regression.

In Chapter 2, we examined model selection techniques in meta-regression. Meta-regression associates the characteristics of the primary studies whose results are synthesized in the meta-analysis to the effect sizes using regressiontype methods. Addressing such associations helps to explain the heterogeneity among the effect sizes that arises from varying study characteristics. However, it is crucial to identify the true model that provides the best approximation to the mechanism that underlies the data. Although common model selection techniques based on testing are applicable, their quality is debatable mostly due to their multiple testing nature. On the other hand, information-theoretic techniques are also applicable in the context of meta-regression which may circumvent the issues in the applications of selection via testing techniques. Furthermore, there is a debate on the likelihood estimation techniques. Some argue that restricted maximum likelihood (REML) estimation is not appropriate for comparing models that differ in their fixed effects; thus, maximum likelihood (ML) estimation should be preferred. Chapter $\mathbf{2}$ demonstrates the applications of all model selection methods in an illustrative example and provides a simulation study to compare their performance under a variety of conditions. The results imply that selection via testing techniques is largely outperformed by information-theoretic approaches. The latter identify the true model with higher chances than conventional techniques. Moreover, REML estimation has a similar or better probability of identifying the true model than ML estimation. Therefore, the results of this chapter suggest the use of information-theoretic model selection techniques more widely than the conventional techniques in the context of meta-regression.

Next, Chapter 3 presents a simulation study to investigate meta-analytic models on correlated effect sizes, specifically in ecology. Regular meta-analytic models do not address two issues that commonly arise in ecology. First, ecology studies mostly examine multiple species that share a common evolutionary history known as a phylogeny which violates the independence assumption in regular meta-analytic models. Second, ecology studies usually report multiple effect sizes which introduces within-study dependence. As regular meta-analytic models assume one effect size per study, this phenomenon is also ignored, and summarizing effect sizes within studies is somehow a questionable strategy that may lead to severe information loss. A phylogenetic multilevel meta-analytic 
model has been proposed in the literature that potentially addresses these issues by i) decomposing the between-species variance into non-phylogenetic and phylogenetic random-effects components and ii) adopting a multilevel framework to incorporate a study-level random effect. Although both of these issues can be addressed within this framework, whether it can provide an advantage over regular meta-analytic models is unknown. For example, the complexity of such a model may pose a threat to model convergence which is why some meta-analytic studies include only the phylogenetic variance component to reduce the model's complexity. Our simulation results show that the overall mean can be estimated with a little or no bias with both regular and complex models. However, it is essential to employ the most complex model to estimate the uncertainty in the overall mean estimation to derive rejection rates close to the nominal level. In addition, the complex model is also successful in estimating the variance components unbiasedly given that there is at least a moderate level of phylogeny. An important finding regarding this issue is that the simplified model that does not include the non-phylogenetic variance component tends to overestimate the phylogenetic variance component, leading to highly conservative results. Finally, our results show that the complex model can be fitted with little or no convergence issues, making it easily applicable. Therefore, the study in Chapter $\mathbf{3}$ shows that the complex multilevel model should be preferred in ecological meta-analyses.

Chapter 4 switches the focus to the combination of dependent $p$-values and its application in genetics. Genome-wide association studies (GWAS) examine the genetic contribution to a disease by examining the association between single-nucleotide polymorphisms (SNPs) and some phenotype of interest. Nowadays, more than a million SNPs are easily genotyped in GWAS, and testing such a large number of SNPs simultaneously inflates the Type I error rate quickly. Controlling the Type I error rate with conventional methods such as the Bonferroni correction reduces the study power severely due to the large number of simultaneous tests. An alternative analysis to circumvent this issue is gene-based testing which shifts the focus of the study to the gene-level where the number of simultaneous tests can be decreased dramatically. Gene-based testing combines the evidence of individual SNPs that belong to a gene which can statistically be performed by combining their $p$ values. Although there are well-known methods for combining $p$-values, they usually assume independence among the $p$-values which is violated due to the non-random associations among SNPs known as linkage disequilibrium (LD). Several techniques are available to adjust the methods for combining $p$-values for dependence among the $p$-values. In Chapter 4, we review the most well- 
known statistical methods for combining $p$-values and adjustment techniques for dependency and provide a simulation study to compare their performance under a variety of conditions. Our results show that incorporating the LD information is essential for controlling the Type I error rate. The methods based on multiple testing adjustments (i.e., the Bonferroni and Tippett methods) are overly conservative, while the other techniques under examination are liberal. A generalization of Fisher's method, known as Brown's method, is the most successful technique to control the Type I error rate while retaining sufficient power; however, it is also essential to use its proper generalization for two-sided tests. Brown's method also has a robust performance regarding the number of SNPs and the degree of LD between them in a gene. On the other hand, empirical techniques that do not require a full permutation procedure also perform adequately and, along with Brown's method, provide a large benefit compared to the commonly used Bonferroni method.

Finally, Chapter 5 introduces the poolr package for the open-source statistical analysis software $\mathrm{R}$ that implements the methods for combining dependent $p$-values investigated in Chapter 4. Furthermore, the poolr package implements a method based on the inverse chi-square distribution. The package implements adjustment techniques based on empirical distributions (also included in the simulation in Chapter 4) and shows that these adjustments can mimic the results that would be obtained by proper permutation-based methods much quicker. Chapter $\mathbf{5}$ presents the use of the poolr package with illustrative examples which can be used as a tutorial for researchers. 


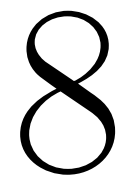

Impact on Society 
Meta-analysis is a highly beneficial tool for researchers to synthesize information from primary studies in various scientific fields. By synthesizing the primary results in a principled way, meta-analysis, in theory, promises results that are more precise and generalizable than the results reported in primary studies. However, a meta-analysis should be conducted carefully by addressing data dynamics that may threaten the quality of the results. Chapter $\mathbf{2}$ and Chapter $\mathbf{3}$ focuses on two such aspects and provide suggestions for improving the quality of future meta-analyses. The former chapter provides suggestions for model selection in meta-regression that can help to identify the true model and explain the heterogeneity among the effect sizes, whereas the latter emphasizes the advantages of using a specific complex model that can capture the uncertainty in the estimated overall meta-analytic mean when there is dependency among the effect sizes, a common dynamic known as phylogeny in ecology. Therefore, these two chapters will hopefully be helpful to future meta-analysts to improve the quality of their studies. In addition to meta-analysis, another issue discussed in this dissertation are genome-wide association studies. This type of study is commonly used in research to examine the contribution of people's genetic background to the risk of developing certain diseases. Chapter 4 reviews methods that could improve the ability of such analyses to detect relevant genome regions with an analysis type known as gene-based testing. The results of this chapter will hopefully help future analysts to improve the power of their studies. Furthermore, Chapter $\mathbf{5}$ introduces an open-source and free to use software package that implements the methods reviewed in the previous chapter. The software introduced in this chapter will enable the analysts to use such methods that are likely to be useful in a wide range of scientific fields. 


\section{Acknowledgments}

I would like to begin with thanking to my supervisor, Wolfgang Viechtbauer. Thank you very much for everything you have taught me, Wolfgang. It was so much fun to learn from you. Also, I have learned a lot of exciting, fun things in statistics with you. Our weekly meetings were always great times that I have always looked forward for the next week's meeting just after one is over. And, I always felt better after our meetings. I feel so lucky to have such a friendly mentor like you. I really hope you have had a good time too. Thank you very much for everything, I really appreciate them.

I need to thank to Ruud van Winkel because he actually initiated this $\mathrm{PhD}$. I wish we had a chance to work on what we wanted at the beginning, Ruud. Thank you very much for giving me this chance.

I thank to my committee members, Prof. Koen Schruers, Prof. Marcel van Assen, Dr. Math Candel, Prof. Michael Dewey, and Dr. Marjan Drukker for their assessment of my thesis and feedback.

Thanks to Sinan Gülöksüz for the studies we worked on together. Also, thank you very much for your help and support during my time here.

Very warm thanks to Shinichi Nakagawa for giving me the chance to come to Sydney and work with him. I was very lucky to get a chance to observe and learn from a great scientist like you. Thank you very much for your support and encouraging words.

My very special thanks to my supervisors from my Master's degree, Özlem İlk Dağ and Cem İyigün. They have always helped and supported me. Without Cem İyigün's supportive talks, I could not even be here. Without Özlem İlk Dağ, I would miss a great example to follow. Thank you so much.

Members of the Department of Psychiatry and Neuropsychology: Maarten, Patrick, Inge, Anne, Claudia, Aleida, Johan, Tim, Sanne, Ron, Jo, Ine, Jolanda, Trees, Marjan, Ger, Philippe, Uli, Inez, Nicole, Sinan, Thérèse, Jim, Bart, Koen, Lies, Dennis, Wolfgang, Machteld, Nele J., Nele S., Bea, Nele V., Debora, Truda, Karel, Wendy, Peter, and Dafne. Thank you for your hospitality and puatience with me. I felt at home among you and I was very happy to be your colleague. I wish I was a more friendly person so that I 
could return your favor to me. I wish I could speak Dutch. I will miss you and hope the best for all of you.

Beautiful PhD's while I was doing mine: Annelie, Yori, Ehsan, Stijn, Lotta, Silvia, Suzanne, Simone, Thomas, Zuzana, Henni, Steven, Laila, Iris, Jindra, and Esther. Thank you very much foru having me as your friend. Your friendship definitely made me feel safe and connected to here. Lunch times were always fun. I have had some nice conversations there. It was also fun listening to your conversations as I am not much of a talker. Tabletop game nights were so much fun. Video game nights were even more fun. Thanks for all those great times. I am so glad to get to know you all. Also, Mary, Naomi, Jette, Christian, Sophie, Stella, Samantha, Maud, Nikita, Chaira, and Emma. Your friendship and subtle support helped me go through the last part of my time here. It has been a pleasure to know you all. Thank you very much for everything. I hope all the best for you.

Special thanks to my roommate, Boris. I am so glad to share a room and also collaborate with you. Boris is a great guy and a great father. When he sees his analyses' results, he says "interesting". He is also very funny, ffs. I wish you a fantastic future with your wife and kids, Boris. Thanks.

My first friends here in Maastricht, my roommates in the flexroom, Anna, Buket, and Maider. You have been great friends to me. I miss you so much.

And, dear Simone. I personally think that your name could have easily been shown as my co-supervisor in this book. Without your support, achieving this would be much harder if not impossible. Thank you so much for everything. Also, Mathilde, Herm, Dennis, Alexandra, Rebecca, and Joshua; thank you very much for making me feel like I have a family here. You are all so dear to me.

This part is going to be weird because most likely they will never see these words. But, I feel I need to acknowledge them. I thank to every musician I have listened during these years. They literally helped me going through all the bad times -being a cleansing from bad feelings and thoughts for me. Again, this will feel very awkward, but I have to acknowledge especially some names, and show my infinite love and respect to them: Jason Göbel, Sean Malone, Paul Masvidal, Sean Reinert, and Chuck Schuldiner. Thank you for your presence in my loneliness and showing me a way to follow. Thank you for being good people, spreading the bubble of goodness, and showing me that it is so important and precious to try to be a good person. 
Before I go to my family, my final and special thanks to my doctors at MET ggz. I appreciate all your help to keep me going on, especially on behalf of my family. I felt safe there and I love you all. Thank you.

And, my family. My mom, dad, and sister are all my motivation in being alive. Without them, I am nothing, empty. So is this book. I am not good at showing my love. But, I love my family more than anything. I wish everyone would have a family like mine. Also, all our relatives (past and now), my grandparents, aunts, uncles, cousins, everyone; they are all the best. And, my grandmother. I am sure you would all love her if you knew her. I love and thank them all from the bottom of my heart.

Finally, I want to acknowledge my grandfather who has been an important figure in my life and passed away during my $\mathrm{PhD}$. 



\section{About the author}

Ozan Çınar was born on 11 November 1986 in Ankara, Turkey. He studied at the Department of Statistics in the Middle East Technical University in Ankara and graduated in 2010. Then, he started to work as a research assistant and his Master's degree under the supervision of Özlem İlk Dağ and Cem İyigün at the same department. He finished his Master's studies in June 2013. Then, he moved to Maastricht, the Netherlands for his PhD in 2015 under the supervision of Wolfgang Viechtbauer and Ruud van Winkel. He did a research visit to Sydney, Australia in the summer of 2018 to work with Shinichi Nakagawa. 